\title{
MACIOMOLECULAR conlluXes
}

M. V. DOS, hi, Editor 


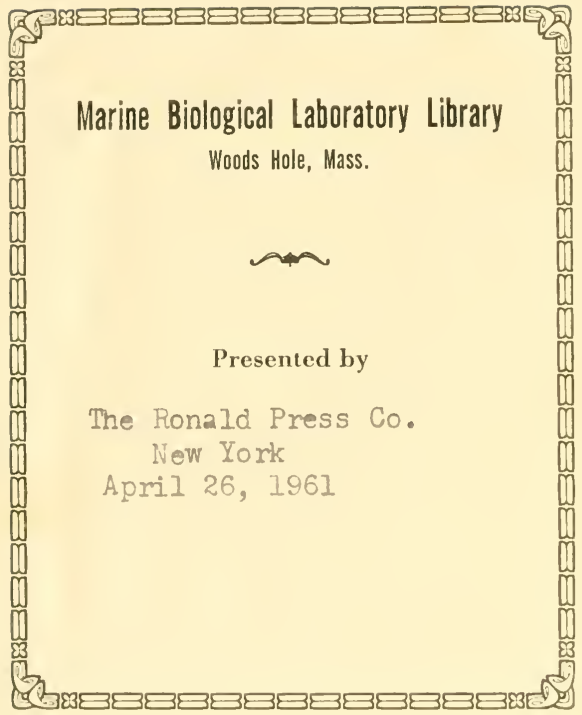




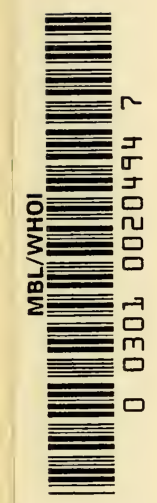




The Society of General Physiologists

Sixth Annual Symposium

University of Illinois

Urbana, Illinois, September, 1959 


\title{
Macromolecular Complexes
}

DAVID F. WAUGH - ALAN J. HODGE - FRANCIS O. SCHMITT MELVIN J. GLIMCHER • J. J. WOLKEN H. FERNÁNDEZ-MORÁN • HANS A. WENT DANIEL MAZIA - J. A. BERGERON • R. C. FULLER WALTER J. NICKERSON • G. FALCONE GIAN KESSLER - R. D. PRESTON

\author{
Edited by \\ M. V. EDDS, JR.
}

BROWN UNIVERSITY

THE RONALD PRESS COMPANY - NEW YORK 
Copyright (C) 1961 by The Ronald Press Company

\author{
All Rights Reserved \\ Nort of this book may be reproduced \\ in any form without permission in writing from \\ the publisher.
}

Library of Congress Catalog Card Number: 61-6324 PIINTED IN TIE UNITED STATES OF AMERICA 


\section{Preface}

Efforts to analyze and interpret life processes, especially as they relate to development, are ultimately confronted with such questions as: Do the orderly patterns constituting given levels of the organizational hierarchy regularly arise solely as a consequence of the specific interactive properties of the units comprising the next lower levels? Given the proper units and the proper conditions, is the spontaneous emergence of order at a higher level inevitable?

Considerable evidence attests that, at least in some cases, these questions may be answered affirmatively. Examples include the in vitro aggregation of certain macromolecules, among which collagen is the prototype, into fibrils of near-crystalline regularity, and the selective aggregation of previously dissociated cells into recognizable tissues. The spontaneous emergence of orderly patterns could also be illustrated by examples from levels higher than the cell or lower than the macromolecule. Between these levels, however, are others about which information is less certain. The time is clearly ripe to begin inquiring into the nature and origin of heterogeneous aggregates of macromolecules, with special emphasis on the interactive properties of their subunits. Actually, the subunits themselves have been identified only in a few cases, and concepts of their structural and functional interrelations are still based largely on inferénce. But, as F. O. Schmitt has written, we have already learned enough to "glimpse, however imperfectly, the portentous possibilities now opening up before us."

Some of these possibilities are explored in this book embodying the proceedings of a symposium sponsored by the Society of General Physiologists. As the text reveals, exploration of the borderland between the realms of the macromolecular chemist and the functionally oriented electron microscopist has already yielded important information about the organization of living matter; it is thus more than an article of faith that many biological problems can be effectively attacked at this level. Not the least of these prob- 
lems are those related to development. It is obvious, but often neglected, that the genesis of form and function occurs, and must be studied, at all levels of the organizational hierarchy. Most of the attempts to relate chemistry and morphogenesis have stressed entities on both sides of the borderland but have been silent on what lies between.

The basic premise of the symposium was that further clarification of the relation between chemistry and morphogenesis will depend heavily on the analysis of aggregates of two or more species of macromolecules. The speakers, who were selected because their main competence lay neither in chemistry nor in morphogenesis, but somewhere in between, were encouraged to discuss the thesis that many aspects of morphogenesis can be attacked in relatively "simple" systems by exploring in detail the properties of the macromolecular complexes which compose them. To this challenge the speakers responded with solid information, both confirming the thesis and raising a host of new morphogenetic problems. Excursions into other areas were inevitable and welcome. Thus, much was said that is related to development only in the sense that it poses the question: How does it develop? But whatever the bias of individual papers, the entire volume is a record of a combined effort to place a point of view before all who may profit from its contemplation.

This symposium was supported by a P.H.S. Research Grant (RG-6228) from the Division of General Medical Sciences, Public Health Service; the symposium was held at the University of Illinois, September 7-9, 1959.

M. V. EdDs, Jr. 


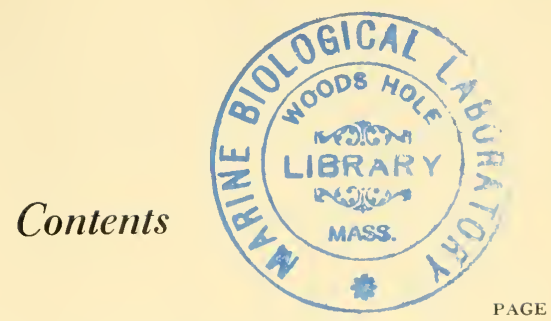

Molecular Interactions and Structure Formation in BioLOgical Systenis

David F. Waugh

The Tropocollagen Macronolecule and Its Properties of Ordered Interaction . . . . . . .

Alan J. Hodge and Francis O. Schmitt

The Role of the Macromolecular Aggregation State and Reactivity of Collagen in Calcification . . . 53 Melvin J. Glimcher

The Chloroplast . . . . . . . . . . J. J. Wolken

Lamellar Systems in Myelin and Photoreceptors as Revealed by High Resolution Electron Microscopy . 113 H. Fernández-Morán

Fibrillar Systenis in the Mitotic Apparatus . . . 161 Hans A. Went and Daniel Mazia

The Submicroscopic Basis of Bacterial Photosynthesis: The Chromatophore 179 J. A. Bergeron and R. C. Fuller 
Polysaccharide-Protein Coxiplexes of Yeast Cell Walls . 205

Walter J. Nickerson, G. Falcone, and Gian Kessler

Cellulose-Protein Complexes in Plant Cell Walls . . 229

R. D. Preston

INDEX . . . . . . . . 255 
Macromolecular
Complexes 



\title{
Molecular Interactions and Structure Formation in Biological Systems
}

\author{
DAVID F. WAUGH ${ }^{1}$
}

The three-dimensional display of structural complexity in cells and tissues, now so convincingly visible in the evidence from electron microscopy, is at the colloidal level, thus the level where one or two dimensions of the particles (rodlets, lamellae) which result from molecular interaction are near the dimensions of the interacting molecules themselves. We accept the fact that the functional aspects of cells, tissues, organs, etc., must be a natural (thermodynamically acceptable) result of the organization at the level mentioned, and, although we are not in a position to state the type or extent of knowledge which will be necessary to understand the way in which subcellular colloidal aggregates modify each other's activities to produce the net result of "life," several aspects of interaction have been examined in a way which must excite all interested in biological processes. These I have been asked to discuss briefly.

Manipulation and interaction at the molecular level involve selections of the interacting molecules; thus, the interactions are specific. We are familiar with the high degree of specificity exhibited by enzyme-substrate interactions (Wilson, 1959), in energy transfer (Lehninger, 1959), in immunological interactions (Landsteiner, 1945), in the formation of tissues such as collagen and elastin (Schmitt, 1959), in the formation of organs, and during the course of embryonic development, to cite a spectrum of examples. As we shall see, specificity of interaction between molecules coming into close contact may be understood on the basis of short-range interactions between appropriately placed submolecular groups of atoms having different interaction characteristics. Catalyzed chem-

\footnotetext{
${ }^{1}$ Massachusetts Institute of Technology, Cambridge, Massachusetts.
} 
ical reactions and the development of the more condensed regions of structure (lamellae, fibrils, etc.) may soon be quantitatively accounted for on this basis.

The biological system is clearly one in which most reactions are carried out in the presence of water. There is, however, apparently an abundance of water, and control of the amount and disposition of water is of paramount importance. The more condensed regions, on whose surfaces or within which much of the molecular traffic will take place, must be prevented from increasing in size (or precipitating out) to the point where such interferes with the execution of ordered series of linked reactions by the condensed structures and with a reworking of the molecules of the condensed structures themselves. We suppose that the incorporation or holding of too much water, however, might well interfere with molecular traffic, would certainly reduce mechanical rigidity, and might allow, for example, deformation of the organ or tissue as a whole to produce local (subcellular) deformations in colloidal structure from which the system would not recover. Control of the amount and disposition of water in localities where the condensed structure is organized in the form of a continuous network (gel) is most readily understood. However, deformation of a cross-linked network must necessarily involve both flow of the entrapped fluid and a rearrangement of the relative positions of the elements of the network. A network arrangement is most effective where the network supports some biological function such as the transmission of tension, as with collagen and elastin. In the examples chosen, the fraction of the tissue or organ occupied by water is low, and the tissues are relatively inert.

Many portions of the biological system, however, contain large amounts of water, and interactions of the more condensed structures may be taking place over intervening aqueous regions of considerable size. Here a distortion of the system need not change the relative positions of the elements of the condensed structures; for example, lamellae might slide past one another without altering the interlamellar distance, thus without altering the local distribution of water and solutes.

In considering molecular interaction and the formation of condensed structures, we must first note that the word "molecule" can be given a restricted meaning with respect to small molecules: namely, a group of atoms joined by covalent (infrequently coordination) bonds which are stable with respect to kinetic energy $(k T)$. 
The fact that covalent bonds have associated with them preferred or limited distances, directions, and rotations in conjunction with the sizes of the participating atoms (Pauling, 1940) means that the possible conformations of the molecule may be specified. As the number of atoms increases, possibilities arise for various types of interaction between groups of atoms within the same covalently linked assembly. In molecules of large size, such as the proteins, carbohydrates, and nucleic acids, these interactions are numerous and involve all known types of forces. For example, these interactions are involved in determining the structures of the polypeptide chain helices of proteins (Pauling ct al., 1951), the interactions of proteins (Waugh, 1954), and the double helix of nucleic acids (Watson and Crick, 1953). Recognition of the importance of the group of short-range forces in determining specificity of interaction, as well as the intramolecular stabilizations just mentioned, is due largely to the work of Pauling and co-workers.

Table 1 gives a general idea of the various types of short-range and long-range interactions. The characteristics of the first of these, the covalent bond, have already been mentioned. The hydrogen bond is a permanent dipole interaction, involving a hydrogen atom covalently bonded to one electronegative atom and the unshared electron pair of another electronegative atom (Pauling, 1940; Orgel, 1959). This bond has a high interaction energy, a result of the small size of the proton which permits the close approach of dipoles. To break a hydrogen bond requires an investment of about $5 \mathrm{kcal}$ per mole. However, if H-bond exchange with water is possible, the investment is much reduced and may be reversed if the groups involved make unusually strong hydrogen bonds with water. Thus the strength of a particular H-bond is dependent on the circumstances permitting a simultaneous interaction with water.

The simple ionic bond, a strong interaction in the absence of water, leads to attraction or repulsion according to Coulomb's law. The interaction energy varies with the dielectric constant of the intervening medium and inversely with the distance between centers. The former varies strongly with distance in an aqueous medium and decreases as the distance between centers decreases (Pressman et al., 1946). Assuming that water hydrates an interacting pair such as an ammonium ion and carboxyl ion, close approach will produce an interaction energy of about $5 \mathrm{kcal}$ per mole (Pauling et al., 1946). We should remember also that charged groups lead 


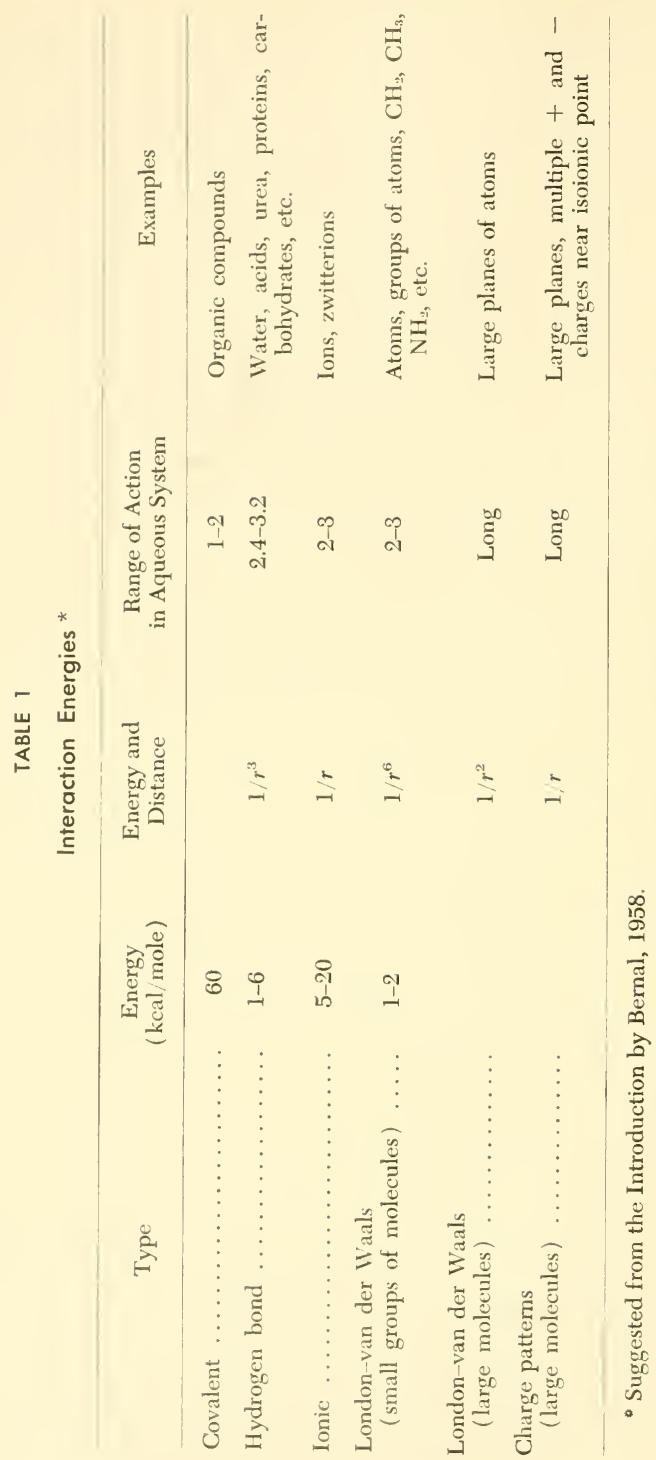


to an ion-dipole interaction with water molecules and so become hydrated, the interaction energy amounting to about 5 kcal per mole of bound water (Dole and McLaren, 1947).

The last of the forces operating at close approach are the Londonvan der Waals attractive forces, which result from the polarization of each group of atoms in the fluctuating electrical field arising from the instantaneous configurations of the electrons and nuclei of adjacent groups. For small groups, the energy varies with the inverse 6 th power of the distance between centers and directly with the molar refractions of the groups involved (Pauling and Pressman, 1945).

\section{Specificity Effected by Short-Range Interactions}

It is of importance to note that both the hydrogen-bond and van der Waals interactions vary, respectively, inversely with the $3 \mathrm{~d}$ and 6 th powers of the distance of separation of the groups. If the maximum interaction energy is released when, for example, two $\mathrm{CH}_{2}$ groups are $4 \AA$ apart, separating these groups by an additional $2 \AA$ will reduce the interaction energy by a factor of about 11 . Likewise for the hydrogen bond, stretching the bond by $1 \AA$ essentially terminates the interaction. We see at once an extraordinary specificity can be accounted for by the requirement that an adequate interaction energy be obtained only when a variety of groups having different interaction characteristics are brought into their proper positions. These groups would include unlike charges, those forming hydrogen bonds, and the bulky groups, interacting through van der Waals forces. The latter, which will essentially define the surface of a large molecule, must fit so that the protuberances on one surface find appropriate cavities on the other surface. Remembering that a stable interaction is produced when the total interaction energy is about 10 to $15 \times k T$, and that all local or group interaction energies are summed to obtain the total interaction energy, suggests that the smaller the area of interaction, the more perfect the fit between interacting surfaces or the more numerous the groups giving rise to stronger interactions.

Even the most specific of interactions can now be rationalized on the basis given; for, as is evident, minute alterations in structure 'may lead to imperfect steric fit, and since energies of inter- 
action are so sensitive to distance, a small imperfection in fit produces a large change in interaction energy.

A product of the short-range interaction between two molecules might well be a complex having new properties. A clear case of this type is the dissociation or fragmentation of the protein ribonuclease into two enzymatically inactive portions (Richards and Vithayathil, 1959). Although the fragments have been produced by hydrolyzing a covalent bond, the fragments, on mixing, combine to give a complex which has full enzymatic activity. The ruptured covalent bond is not re-formed on mixing. A variety of exciting possibilities is brought to our attention by this discovery. For example, the interaction of a steroid hormone molecule with an inactive protein might induce enzymatic activity in the latter, this combination controlling a host of subsequent reactions.

Specific interactions are undoubtedly also involved in establishing condensed regions, which usually appear as filaments, lamellae, or helices, at levels of size within an order of magnitude of $100 \AA$. Currently, the lamellar structure containing lipid and protein appears to be a conspicuous component of cells, the assumption being made that the lipid within the lamella will be present as a double layer or layers. Certainly such an organization of lipid would be expected from the properties of soaps and lipid emulsions (Luzzati et al., 1958; Lawrence, 1958; Palmer and Schmitt, 1941) and the structure of the myelin sheath (Schmitt, 1959). The double-layer arrangement permits the bulky non-polar portions of the lipid molecules to associate, leaving surface layers of polar groups to interact with each other and with water, protein, or other polar substances.

In many instances the development of a lamella may not involve any high degree of fit (specificity) of the polar and non-polar portions of the molecules involved. We would expect a low degree of specificity mainly when the interaction leads to the development of a structure which carries out some non-critical physical function, a safe example being that of fat storage-although another might be the essentially two-dimensional expansion of non-functional portions of a plasma or other membrane. Certain functional aspects, however, may require a specific arrangement of non-polar and polar portions which will provide an energy barrier to penetration (permeability). Although the physical conditions establishing the basis for interaction in monomolecular films are probably not duplicated often in biological systems, the effects of short-range interaction in 
modifying the properties of films are clearly shown in studies of the evaporation of water through compressed films of stearic acid or cetyl alcohol; the latter present an extraordinary energy barrier to the penetration of water molecules (Langmuir and Schaefer, 1943). This possibility, namely, that non-polar portions of molecules, particularly lipid molecules, may interact to present a high-energy barrier to the penetration of water, is of considerable biological interest; for, in spite of the relative abundance of water in biological systems, local regions at the particulate level may involve boundaries between essentially aqueous environments and environments where the interactions of non-polar portions of molecules are so well integrated that water is virtually excluded. A structure of this type, for example, may be necessary in effecting the key reactions of photosynthesis (Calvin, 1959).

In this introductory discussion, I would like to summarize some of our experiences with two protein systems in which short-range interactions, coupled with particular types of specificity, lead either to extended fibrils or to spherical micelles, namely, the interactions of insulin (Waugh, 1957) and of casein (Waugh, 1958).

Mechanism of Fibril Formation. The insulin fibril forms under conditions where the dimer of $\mathrm{M} \sim 11,000$ is the prevalent form, i.e., at $\mathrm{pH}$ 2. Heating at $80^{\circ}$ to $100^{\circ} \mathrm{C}$ causes a spontaneous transformation into a population of fibrils, the most numerous, and largest, of which are about $200 \AA$ in diameter and many thousands of Ångströms in length. The reaction goes essentially to completion but is reversible in the sense that under alkaline conditions the fibril disaggregates to yield insulin. Evidence which gives a clue to the mechanism of fibril formation, and which suggests that the insulin molecule does not undergo extensive unfolding in the process of forming fibrils, comes from experiments in which fibril segments are seeded into insulin solutions at $\mathrm{pH} 2$. While such solutions alone are stable for long periods of time at temperatures of $20^{\circ} \mathrm{C}$ or below, the seeded fibrils or fibril segments recruit insulin from solution and in the process grow according to first-order kinetics. Structurally, the new portions of the fibrils obtained after fibril growth at lower temperatures appear to be identical with those which are formed at $80^{\circ}$ to $100^{\circ} \mathrm{C}$.

When a solution of insulin is heated and the transformation of insulin into fibrils is plotted as a first-order reaction, the resulting curves typically have a lag period which is followed by an essen- 
tially linear rise. The extent of the lag period is determined markedly by the initial insulin concentration, as is the slope of the nearlinear portion of the curve. A comparison of the reaction kinetics observed with the growth of seeded fibrils and in the absence of seeding suggests that the fibril is first initiated by a nucleation reaction which involves the cooperative effects of three or four interacting units (dimers). Thus the rate of nucleation varies with the $3 \mathrm{~d}$ or 4 th power of the insulin concentration. After initiation, when the fibril has achieved a reasonable size, the fibril grows as a function of its surface area and the 1st power of the free insulin concentration. The cooperative effect established during nucleation is perpetuated, and the surfaces (particularly of the ends) of the fibril present to the entering insulin unit the correct cooperative configuration necessary for bonding.

One particularly interesting consequence of this type of mechanism is that most of the fibrils are initiated during the lag period of the reaction. Those which are initiated during the first few minutes of the lag period dominate the reaction, in the sense that they are responsible for removing most of the insulin. The fibril population at the end of the reaction appears to be relatively

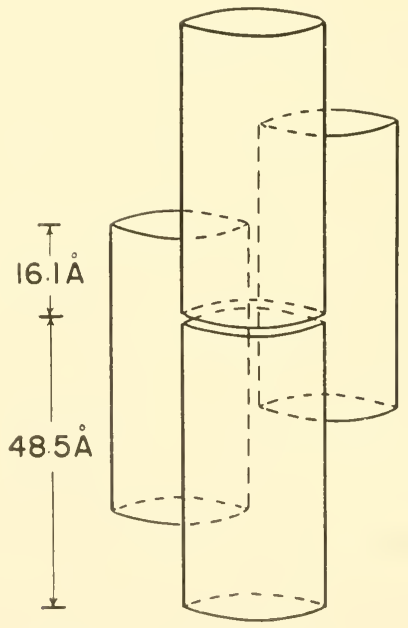

Fig. 1. Diagram of a fibril nucleus, illustrating a cooperative interaction. (From Waugh, 1957.) 
homogeneous. Aggregations of the fibrils themselves may lead to the formation of spherites.

It is clear that, at least over a portion of its development, the fibril axial ratio increases with fibril mass. That an asymmetric aggregate should form when charged molecules link, without specification of surface structure, has been pointed out by Rees (1951). This is because the electrostatic-potential barrier is lowest at the ends of the dimer or other asymmetric unit. However, the cooperative effect itself will exert a strong directional influence. This is illustrated in Fig. 1, which is a possible arrangement for the insulinfibril nucleus. The stable structure is formed when any fourth insulin unit is added to the correct previous group of three. Thereafter, insulins are expected to add most frequently in a manner which perpetuates this stable structure; consequently, the aggregate elongates in the direction of the cylinder axis of Fig. 1.

Stable Colloids. The interactions of the caseins (Waugh, 1958) reveal both specificity of interaction and cooperative effects, the end result in milk being a population of micelles varying in chemical composition and volume, the latter over a range of about 350 fold. Yet the micelles are in rapid equilibrium with their constituent components in solution, some of which are relatively insoluble under the conditions where micelles readily form. The most important initial interaction in micelle formation occurs between $\alpha_{*}$ - and $\mathrm{k}$-caseins, and in this interaction secondary valence and charge interactions arrive at a remarkable mutual satisfaction. Here, $\alpha_{\mathrm{s}}$-casein is a phospho-protein of $\mathrm{M} \sim 23,000$. It has a phosphorus content of over 1 per cent but no disulfide. Physically, this protein appears to be a single coil $210 \AA$ in length and $16 \AA$ in diameter. In the absence of calcium the protein is a highly soluble polymer; the polymer becomes quite insoluble in the presence of calcium. The k-casein is also apparently a single coil about 150 Å long, which contains disulfide but little phosphorus. It forms soluble, condition-insensitive polymers of $13.5 \mathrm{~S}$ in the presence or absence of calcium. When mixtures are made containing molecular ratios of $3 \alpha_{s}$-casein to 1 $\mathrm{k}$-casein, the original polymers disappear and a stoichiometric $\alpha_{\mathrm{s}}$ $k$-casein complex forms. This complex can form in the absence of calcium and under conditions where both of the monomers carry a high 'net negative charge. One must assume that here, as in other cases, secondary valence forces are responsible for association. In this connection, an examination of the amino acid compositions of 
all the caseins reveals a high-occurrence frequency for the larger non-polar amino acids.

The $\alpha_{s}$-k-complex, although it exists at all temperatures between $0^{\circ}$ and $37^{\circ} \mathrm{C}$, is unstable at the lower temperatures. This is apparent from the results obtained on adding calcium to give $0.05 \mathrm{M}$. At the higher temperature, the complexes are stabilized and will now engage in further aggregation among themselves and with $\beta$-casein to form colloidal micelles typical of milk. Once formed, the $\mathrm{Ca}-\alpha_{\mathrm{s}}-\mathrm{k}$-casein complex is unusually stable to heat, changes in $\mathrm{pH}$, etc. We propose for it a structure in which the three $\alpha_{\mathrm{s}}$-casein molecules are oriented axially around a k-casein molecule. At higher temperatures, secondary valence interactions hold (against electrostatic repulsion) the $\alpha_{\mathrm{s}}$-casein molecules in positions such that pairs of phosphorus groups attached to adjacent $\alpha_{s}$-casein monomers are in juxtaposition and can be linked through the introduction of a calcium ion. The precision with which the phosphate groups are positioned is probably dependent upon the conformations of the interactants, for, at lower temperatures, the instability of the complex suggests that the phosphorus groups cannot be cross-linked by calcium. If each phosphorus is able to accept a calcium ion, stabilization through Ca-phosphate cross-linking would be absent, and, since Ca- $\alpha_{\mathrm{s}}$-caseinate is relatively insoluble at low temperatures, it would precipitate and thus lead to a dissociation of complexes.

\section{Long-Range Interactions}

The presence of large amounts of water in certain types of biological systems poses special problems, particularly if the condensed regions of the system in question do not form a continuous network. Two items currently of great interest are the structure of water in such regions and the possibility that long-range forces may be involved in establishing the structure of the system.

The last two items of Table 1 relate to long-range forces. The first of these, listed as London-van der Waals forces, have their origins in mutual polarization, as was stated above. However, the specification that large planes of atoms are interacting with each other, and the fact that these interactions are additive, modifies the variation in attractive energy with distance so that, for molecules or condensed regions presenting sufficiently large interacting surfaces, 
the interaction energy varies inversely with the square of the distance (Overbeek, 1952; Prosser and Kitchener, 1956).

The last item of Table 1 refers to large molecules carrying many groups capable of being positively or negatively charged, or uncharged. At the isoionic point and at low ionic strength, mutual electrical polarizations will tend to establish charge patterns on the two molecules which lead to an electrostatic attractive energy which decreases in proportion to the distance. At short distances, interaction through charge fluctuation might provide an interesting explanation for the participation of the protein moiety of an enzyme molecule in the mechanism of hydrolytic enzyme reactions (Kirkwood, 1957).

Of great interest to the biologist are those systems containing large, usually asymmetric, particles at the correct concentration, $\mathrm{pH}$, and ionic strength which separate into two phases: one phase in which the solute concentration is reduced below average and in which orientation of the particles is absent, and a second phase in which the solute concentration is increased above average and the particles are well oriented and exist in a three-dimensional pattern. Examples are to be found in bentonite (platelets), tobacco mosaic protein (rodlets), and many systems in which "colloids" carrying net charges of different sign are mixed. The last-named examples, which must be of importance in providing an understanding of the way in which large molecules interact over intervening aqueous gaps, have recently been analyzed by Overbeek (1957) and by Michaeli et al. (1957). They treat the system theoretically as a competition between charge interactions, which tend to accumulate the charged particles, and entropy, which tends to disperse them. Of considerable interest are the results obtained when the charged particles, the polyanion and polycation, are present in different concentrations; for now the concentrated phase, the coacervate, will be more symmetric in concentration than the starting mixture, while the dilute phase will be more asymmetric. Separation into phases takes place at solute concentrations which make these systems of immediate biological interest.

The structure of water in biological systems is also a matter of importance. Water of hydration, at least that associated with polar groups, will be firmly bound and will be involved in defining the contour and properties of the surface of the condensed structure to which it is attached. In the lamellar systems mentioned previously 
there is apparently space to accommodate water layers ranging up to about $1000 \AA$ in thickness. The properties of the system will naturally depend on whether this water is solvent water having the properties of bulk water at the same temperature, or water which, by virtue of the structures of the contiguous condensed regions, has a more or less regular arrangement. Szent-Györgyi (1956) has recently considered the importance of water structure and has performed several intriguing experiments which suggest that water in the biological system may have predominantly an icelike structure. Kauzmann (1959), in a review of protein denaturation, discusses the formation of icebergs around non-polar groups, as well as the short-range interactions of interest here. The evidence presented by Kauzmann suggests that the ordering of water molecules is confined to the locality producing the ordering and is not transmitted beyond a few Ångströms.

If we restrict ourselves to considerations of colloidal structure, it is apparent from the foregoing that molecules may, by a variety of short-range interactions, essentially come out of solution and develop condensed regions which, from the principal directions of development, would be classified as rodlets, filaments, lamellae, platelets, etc. One of the systems, involving the development of collagen filaments, not only may illustrate the relationships between size and long-range interaction but suggests the possibility of specific long-range interactions. This system, the basement lamella lining the skin of amphibian larvae, has been treated by Weiss (1957). In the region of the basement lamella there are approximately 20 layers, each $2000 \AA$ in depth. Each layer contains a system of rods, tentatively identified as collagen, which are 500 i in diameter and cross-striated at $520 \AA$ intervals. Within any one layer the rods run parallel, with their cross-striations in lateral register. However, the orientation of each laver differs from that of adjoining ones by an angle of $90^{\circ}$.

If a piece of membrane is excised, the membrane is repaired. During this process there first appear small cross-striated fibrils of about $200 \AA$ diameter. These are initially unoriented, but as they grow in length and diameter the particular matched orthogonal pattern is established. If the medium between the oriented fibrils does not contain condensed structures to transmit directions for orientation and for striation matching, the system strongly suggests the existence of specific long-range interactions. It is possible that, 
as the extension in one or two dimensions becomes sufficiently large, the long-range interactions at separated loci are quite different. Thus, possibly an interaction pattern will emerge which we will regard as being specific.

Without proving the point, a great deal of evidence may be selected from the behavior of biological systems which indicates that long-range interactions may have elements of specificity. The classical example is chromosome pairing, which has long excited interest. I feel that the behavior of chromosomes during meiosis suggests that a specific interaction occurs at the kinetochores, providing a primary point of orientation and possibly overcoming an initial energy barrier to close approach. Thereafter, long-range interactions might align homologous segments. Of course, a few of the up-to-now invisible strands might provide condensed regions over which a continuum of short-range interactions would be responsible for pairing.

We appear to be dealing with fundamental types of forces of interaction involving long- and short-range patterns of interacting groups which can give rise to specificity of size, shape, and chemical differentiation of interacting particles. From the large number of possible combinations, the problem is to isolate and understand those particularly important types which will further our understanding of the way in which structure is developed and of the relationships between structure and function.

\section{References}

Bennett, H. S. 1959. Structure of muscle cells. Revs. Modern Phys. 31: 394-401.

Bernal, J. D. 1958. Structure arrangements of macromolecules. Discussions Faraday Soc. No. 25: 7-18.

Calvin, M. 1959. Energy reception and transfer in photosynthesis. Revs. Modern Phys. 31: 147.

Dole, M., and A. D. McLaren. 1947. The free energy, heat and entropy of sorption of water vapors by proteins and high polymers. J. Am. Chem. Soc. 69: 651-657.

Fernández-Morán, H. 1959. Fine structure of biological lamellar systems. Revs. Modern Phys. 31: 319-330.

Hodge, A. J. 1959. Fibrous proteins of muscle. Revs. Modern Phys. 31: 409-425.

KaUzManN, W. 1959. Some factors in the interpretation of protein denaturation. Advances in Protein Chem. 14: 1-57.

Kirkwood, J. G. 1957. The forces between protein molecules in solution. J. Cellular Comp. Physiol. 49 (Suppl. 1): 59-62.

LANDSTEINER, K. 1945. The Specificity of Serological Reactions. Rev. ed. Harvard University Press, Cambridge, Mass.

Langmuir, I., and V. J. Schaefer. 1943. Rates of evaporation of water through compressed monolayers on water. In F. R. Moulton (ed.). Surface Chemistry. Publ. Am. Assoc. Advance. Sci. No. 21. Pp. 17-39. 
Lawrence, A. S. C. 1958. Solubility in soap solutions. Part 10. Phase equilibrium, structural and diffusion phenomena involving the ternary liquid crystalline phase. Discussions Faraday Soc. No. 25: 51-58.

Lehninger, A. L. 1959. Respiratory-energy transformation. Revs. Modern Phys. 31: 136-146.

Luzzati, V., A. Mustachoi, and A. Skoulios. 1958. The structure of the liquidcrystal phases of some soap + water systems. Discussions Faraday Soc. No. 25: 43-58.

Michaeli, I., J. Th. G. Overbeek, and M. J. Voorn. 1957. Phase separation of polyelectrolyte solutions: J. Polymer Sci. 23: 443-450.

Orgel, L. E. 1959. The hydrogen bond. Revs. Modern Phys. 31: 100-102.

Overbeek, J. TH. G. 1952. London-van der Waals attraction between two parallel flat plates. In H. Kruyt (ed.). Colloid Science. Elsevier Publishing Co., Amsterdam. Vol. 1, pp. 266-268.

Overbeek, J. Th. G. 1957. Phase separation in polyelectrolyte solutions. Theory of complex coacervation. J. Cellular Comp. Physiol. 49 (Suppl. 1) : 7-26.

Palmer, K. J., and F. O. Schmitt. 1941. X-ray diffraction studies of lipide emulsions. J. Cellular Comp. Physiol. 17: 385-393.

Pauling, L. 1940. The Nature of the Chemical Bond. 2d ed. Cornell University Press, Ithaca, N. Y.

Pauling, L., D. H. Campbell, and D. Pressman. 1943. The nature of the forces between antigen and antibody and of the precipitation reaction. Physiol. Revs. 23: 203-219.

Pauling, L., R. B. Corey, and H. R. Branson. 1951. The structure of proteins: Two hydrogen-bonded helical configurations of the polypeptide chain. Proc. Nat. Acad. Sci. U. S. 37: 205-211.

Pauling, L., and D. Pressman. 1945. The serological properties of simple substances. IX. Hapten inhibition of precipitation of antisera homologous to the o-, m-, and p-azophenylarsonic acid groups. J. Am. Chem. Soc. 67: 1003-1012.

Pressman, D., A. L. Grossberg, L. H. Pence, and L. Pauling. 1946. The reactions of antiserum homologous to the p-azophenlytrimethylammonium group. J. Am. Chem. Soc. 68: 250-255.

Prosser, A. P., and J. A. Kitchener. 1956. Direct measurement of long-range van der Waals forces. Nature 178: 1339-1340.

ReEs, A. L. G. 1951. Directed aggregation in colloidal systems and the formation of protein fibers. J. Phys. Chem. 55: 1340-1344.

Richakds, F. M., and P. J. Vithayathil. 1959. The preparation of subtilisin-modified ribonuclease and the separation of the peptide and protein components. $J$. Biol. Chem. 234: 1459-1465.

Scumit, F. O. 1959. Interaction properties of elongate protein macromolecules with particular reference to collagen (tropocollagen). Revs. Modern Phys. 31: 349-358.

Sjöstrand, F. S. 1959. Fine structure of cytoplasm: The organization of membranous layers. Revs. Modern Phys. 31: 301-318.

Szent-Györgyi, A. 1956. Bioenergetics. Science 124: 873-875.

Watson, J. D., and F. W. C. Crick. 1953. Molecular structures of nucleic acids. Nature 171: 737-738.

WaUG11, D. F. 1954. Protein-protein interactions. Advances in Protein Chem. 9: $325-437$.

WAUG11, D. F. 1957. A mechanism for the formation of fibrils from protein molecules. J. Cellular Comp. Physiol. 49 (Suppl. 1): 145-164.

IVAUGI, D. F. 1958. The interactions of $\alpha_{s}, \beta$ - and k-caseins in micelle formation. Discussions Faraday Soc. No. 25: IS6-192.

Weiss, P. 1957. Macromolecular fabrics and patterns. J. Cellular Comp. Physiol. 49 (Suppl. 1): 105-112.

Wilson, I. B. 1959. Molecular complementarity and antidotes for alkylphosphate poisoning. Federation Proc. 18: 752-758. 


\section{DISCUSSION}

\section{A. G. Richards, D. F. Waugh, W. D. McElroy, W. J. Nickerson}

Dr. Richards (University of Minnesota): During development of an insect's cuticle, there is a large dehydration of the chitin-protein matrix and a simultaneous loss of almost all the salt, as shown by microincineration. Does this imply that the salts, which are not homogeneously distributed in the cuticle matrix, are nevertheless present effectively only in free solution in the water phase?

Dr. WAUGH: The salts which are distributed heterogeneously are probably not present effectively in free solution in the water phase. If we were sure that there were no restrictions to diffusion and no active transport, the statement could be made more positively. If this were so, it would appear that during dehydration the binding sites responsible for heterogeneity effectively disappear, leaving the ions to be swept out.

Dr. McElroy (Johns Hopkins University): Is the water between fibrils at such a low concentration that a limited chemical reaction could remove essientially all of it? Are there good examples from biological structures in which the primary forces (van der Waals, H-bond, etc.) are only important in bringing the molecules together? Do other chemical processes then occur, which are important in giving the final property to the structure?

Dr. WAUGH: What kinds of fibrils? Usually there is too much water to be removed by a limited chemical reaction. In this introduction, I have treated the forces which are most likely to be operating in determining specificity of interaction and structure formation. The final properties of the structure will undoubtedly involve types of interactions and, particularly, chemical processes not mentioned here; for example, the chemical processes involved in photosynthesis and bioluminescence.

Dr. Nickerson (Rutgers University): Following up on the second of Dr. McElroy's questions, do you have any information on the mechanism whereby a trace of saturated fatty acid glyceride can induce almost instantaneous crystalline order in a liquid, unsaturated fatty acid glyceride? This reaction appears, from some work in our laboratory on development of yeast cells, to have a biological reality.

Dr. WaUgh: The fatty glycerides undergo a series of phase changes with temperature, and a sizable literature is available on this subject. It is possible that a small amount of saturated fatty acid glyceride induces crystallization by accelerating the formation of crystal nuclei. 



\section{The Tropocollagen Macromolecule and Its Properties of Ordered Interaction ${ }^{1.2}$}

Alan J. Hodge ${ }^{3}$ and Francis O. Schmitt ${ }^{4}$

The discovery that collagen in solution exists in the form of discrete monomeric macromolecular units, termed "tropocollagen" (TC) by Gross et al. (1954), and consists, in all probability, of several polypeptide chains arranged in a specific coiled configuration, has had important consequences in the investigations of the chemistry of connective tissue, in the biomedical field, and in fields of special application such as the chemistry of leather. The detailed examination of the various ordered aggregation states of the TC macromolecule in the electron microscope and, in particular, the examination of the so-called "segment long-spacing" form (SLS), which is in effect a "molecular fingerprint" in that it indicates the distribution of polar side-chains, have resulted in the formulation of a mechanism for the end-to-end linkage of the macromolecules, a process presumably involved in fibrogenesis in vivo. The precise packing arrangements of $\mathrm{TC}$ in the various ordered aggregation states are now known in detail, and it is on the basis of the specific stereochemical configurations of the polar side-chains in these various forms that a mechanism for calcification has been advanced by Glimcher et al. (1957). The experimental evidence indicated that only the specific steric arrangement present in the "native-type" aggregation state was capable of initiating nucleation of hydroxy-

${ }^{1}$ The substance of this paper was also presented by one of us (F.O.S.) at the Sixth Congress of the International Union of The Leather Chemists Society, Munich, Germany, September 8, 1959 (Schmitt and Hodge, 1960). The German translation was published in Das Leder.

${ }_{2}$ This investigation was supported by a research grant, E-1469, from the National Institute of Allergy and Infectious Diseases, National Institutes of Health, U. S. Public Health Service.

${ }^{3}$ California Institute of Technology, Pasadena, California.

${ }^{4}$ Massachusetts Institute of Technology, Cambridge, Massachusetts. 
apatite from solutions in metastable equilibrium with respect to calcium and phosphate ions. There is reason to believe, also, that the knowledge thus gained of the TC macromolecule and of its polymerization properties may prove useful in the elucidation of connective tissue abnormalities such as characterize certain of the rheumatoid diseases, atherosclerosis, and aging processes.

\section{The Tropocollagen Macromolecule}

The existence of a discrete type of collagen monomer extractable from a variety of connective tissues under appropriate conditions has been amply confirmed by a variety of techniques. Since both small-angle x-ray diffraction (Bear, 1952) and electron microscope studies on native fibrous collagen indicated a highly ordered band structure with a fundamental repeat of about $640 \AA$, it was assumed by many that the monomer must have a length of this dimension. The first indication that this was not the case came with the observation that soluble collagen could be reconstituted into "longspacing forms," which exhibited axial periods of several times the value characteristic of native fibrils (for summaries, see Schmitt et al., 1955; Schmitt, 1959; Hodge, 1959b). More recent measurements (Hodge and Schmitt, 1960) show very sharp distributions around length values of about $2800 \AA$ for these long-spacing forms, corresponding to four times the native period, and comparably sharp distributions for native-type fibrils. This value is in good agreement with the results of Boedtker and Doty (1956), who found by physicochemical methods that the macromolecules of soluble collagen are highly asymmetric stiff rods with dimensions of about $14 \AA \times 2900 \AA$, and with those of Hall and Doty (1958), who utilized an improved shadow-casting technique for direct visualization of macromolecules in the electron microscope.

The large-angle $x$-ray diffraction pattern of collagen is one of its most striking characteristics and has been interpreted (see Rich and Crick, 1958) as indicating the presence, over large regions of the fibrous structure, of a three-stranded helical configuration of polypeptide chains which involves the sequence glycine-proline-hydroxyproline well known from degradative experiments, the whole structure being stabilized by hydrogen bonding. The three-stranded model is also supported indirectly by the observ 7 ions of Doty and Nishihara (1958) on the denaturation of soluble collagen. On de- 
naturation, the original macromolecules, of weight $c a .360,000$, yield one chain of weight $c a .120,000$ and another of weight $c a .240,000$ (see also Orekovitch and Shpikiter, 1958). According to Doty and Nishihara, the larger of these components in calfskin collagen may comprise two chains linked by an alkali-labile ester bond.

The physical properties of collagen solutions and the other observations already cited leave little doubt that the tropocollagen macromolecule, ca. $14 \AA \times 2800 \AA$ in dimensions, is indeed the monomeric unit of the various forms of ordered aggregates and must possess a high degree of configurational order over at least the major part of its length. A striking property of native collagen macromolecules is their capacity to aggregate under appropriate conditions to yield a number of highly characteristic ordered structures, the type of pattern obtained depending on the environmental conditions. These various ordered aggregation states are characterized by the highly specific band patterns they exhibit when observed in the electron microscope, especially if the contrast is enhanced by treatment with an "electron stain" such as phosphotungstic acid (PTA). The ability to form such ordered structures is lost if the macromolecules are denatured by thermal or other means. It seems, therefore, that the polar side-chains responsible for the banding seen in the electron microscope must be in a rather precise stereochemical array, which is maintained in the native macromolecule by hydrogen-bonding between the constituent polypeptide chains, and which is lost when these bonds are ruptured during denaturation. As we shall see, the polar groups (both basic and acidic) are located in clusters at discrete loci along the length of the TC macromolecule and are separated by regions apparently poor in or lacking polar groups. It is likely that these non-polar regions are mainly responsible for the characteristic large-angle $\mathrm{x}$-ray diffraction pattern of collagens. On the other hand, there seems to be no doubt that the small-angle pattern is the direct result of the particular distribution of the polar side-chain clusters (see Bear and Morgan, 1957). Chemical analytical and electron microscope studies on collagen fibrils by Kühn et al. (1957) and Kühn (1958) indicate that the basic and acidic residues are in the same loci (bands) in the native collagen structure. Their work shows that, while the lysine-bound PTA is easily washed out, that held by arginine is firmly bound and is responsible for the characteristic band pattern and, further, that the rather large phosphotungstate ion is bound 
by the mutual cooperation of several polar side-chains on $c$ djacent macromolecules. It may reasonably be postulated, therefore, that bands will be observed only when the macromolecules retain their native helical configuration and will not be observed in those regions where the polypeptide chains are uncoiled or otherwise disordered.

\section{Aggregation States of Tropocollagen}

For reasons that will become clear after consideration of the various types of band pattern encountered, the TC macromolecule may be considered as an "asymmetric" or "structurally polarized" unit; i.e., the distribution of density along its length is such that one end is always clearly distinguishable from the other. The macromolecule may therefore be represented formally as an arrow, the head and tail being labeled A and B, respectively (e.g., Figs. $1-3)$. This kind of symbolic representation has proved to be useful for indicating the packing of $\mathrm{TC}$ in the various ordered aggregation states.
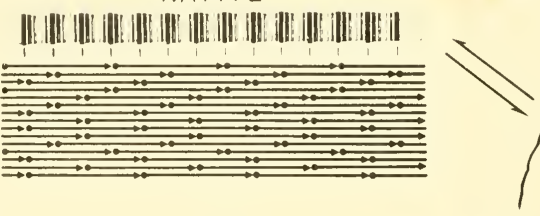

FLS
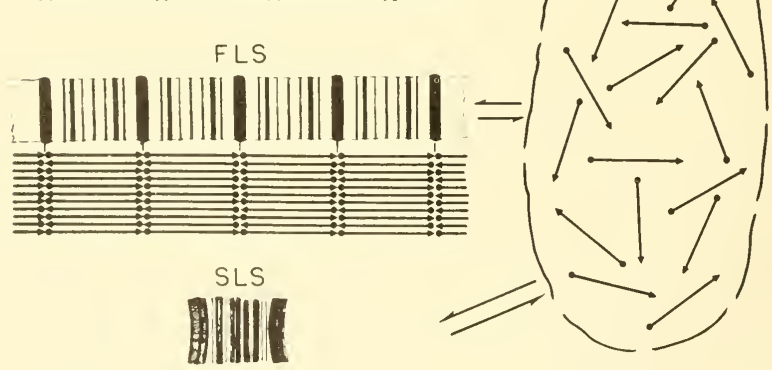

B

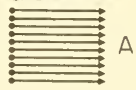

Fig. 1. Diagrammatic representation of three probable modes of aggregation of the TC macromolecule which can be formed reversibly from dilute acid solutions of TC under appropriate conditions. See text for description. 


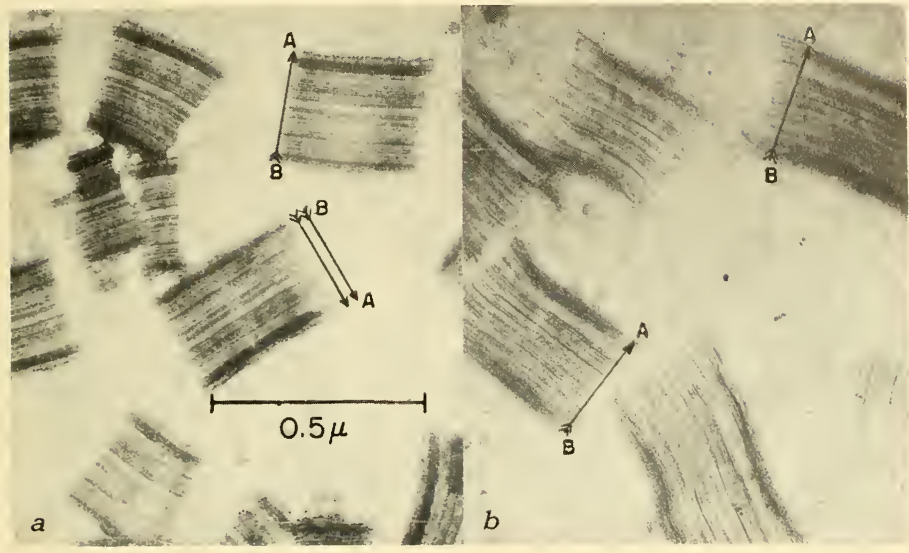

Fig. 2. Band patterns of SLS-type aggregates obtained by addition of ATP to an acid solution of calfskin collagen. In this ordered form, the TC macromolecules are packed in "parallel array," with like ends in register, and their orientation is indicated by the arrows with ends labeled A and B. (a) Stained with PTA. (b) Stained with cationic uranium. $\times 60,000$. (From Hodge and Schmitt, 1960.)

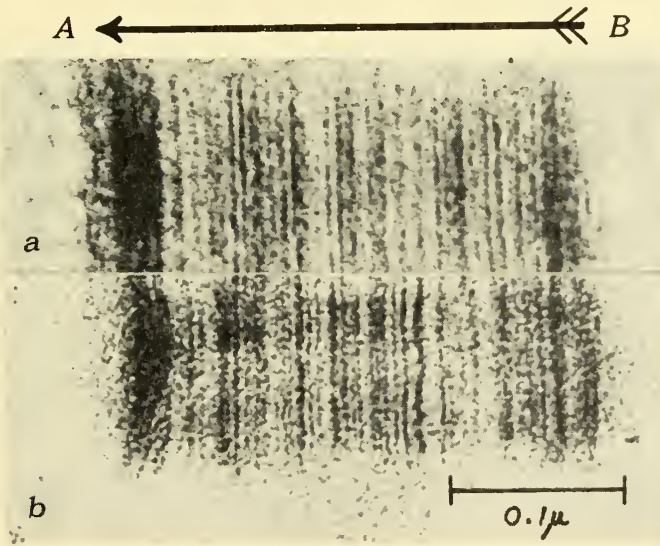

Fig./ 3. SLS-type aggregates, showing the close correspondence in the location of staining loci in the two "fingerprints" despite considerable differences in staining intensities. (a) Stained with PTA. (b) Stained with cationic uranium. $\times 220,000$. (From Hodge and Schmitt, 1960.) 
The simplest "crystalline" form of collagen is that which Schmitt et al. (1953) termed segment long-spacing (SLS), and is readily formed on addition of adenosine triphosphate (ATP) to a dilute acid solution of TC, although segments can also be formed under other conditions. In this aggregation state the macromolecules are arranged in a parallel array with like ends and other features in register (Figs. 1-3).5 The asymmetric or "polarized" band pattern observed when SLS is stained with PTA is thus, in effect, a "molecular fingerprint," since it directly indicates the distribution and concentration of basic side-chain groups ${ }^{6}$ along the length of the TC macromolecule. A comparable "fingerprint" of the acidic sidechains (Fig. 2) may be obtained conveniently by staining the segments with cationic uranium under appropriate conditions (Hodge and Schmitt, 1960).

The end regions of individual segments are generally somewhat disordered, and a detailed comparison of the staining properties of

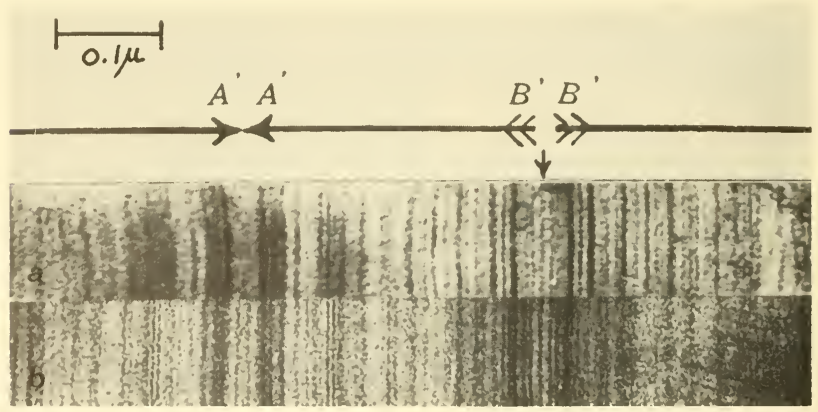

Fig. 4. Polymeric SLS-type aggregates obtained by addition of ATP to collagen solutions subjected to sonic irradiation (see text). In these polymeric forms, the distortions due to drying are minimized, particularly for staining loci near the ends of the TC macromolecules, and thus allow a better comparison of the various band densities. (a) Stained with cationic uranium. (b) Stained with PTA. $\times 135,000$. (From Hodge and Schmitt, 1960.)

${ }^{5}$ Unless otherwise indicated, all the illustrations have reference to preparations of calfskin collagen.

"It should be understood that the actual band densities observed in the electron micrographs represent both an "intrinsic electron density," resulting from the concentration of the bulky polar side-chains into clusters along the length of the TC macromolecule, and an electron density contribution arising from the binding of heavy metal ions. 
bands in these regions is more conveniently obtained from observations on certain polymeric segment-type aggregates obtained from solutions of collagen treated with sonic irradiation (see Fig. 4). It is of interest that the two "fingerprints" thus obtained match each other exactly in terms of the relative positions of the forty or so

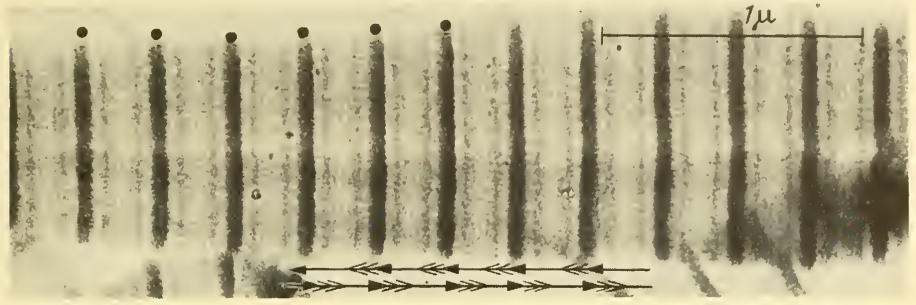

Fig. 5. FLS-type fibril with an axial period of ca. $2800 \AA$. The symmetrical intraperiod band pattern corresponds with that expected for a packing of protofibrils (linear polymers of TC) having no preferred polarization, i.e., in antiparallel array, and with A-B junctions in approximate register. Stained with PTA. $\times 33,000$. (From Hodge, 1959b.)
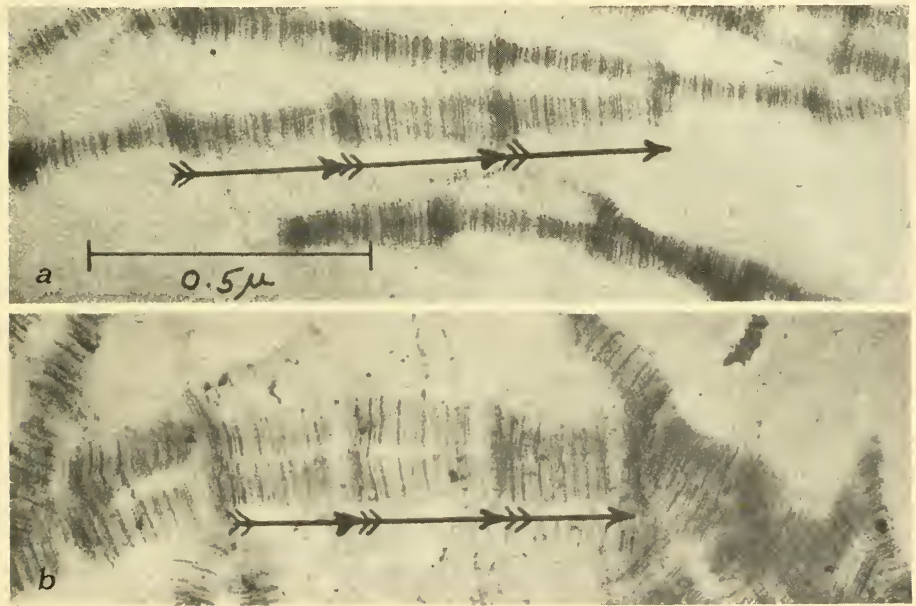

Fig. 6. Polymeric SLS-type fibrils produced by addition of ATP to an acid solution of collagen at relatively high $\mathrm{pH}$ (ca. 5). In this form, the linear polymers of TC aggregate with all like features in register. (a) Stained with PTA. (b) Stained with cationic uranium. $\times 70,000$. (From Hodge and Schmitt, 1960.) 

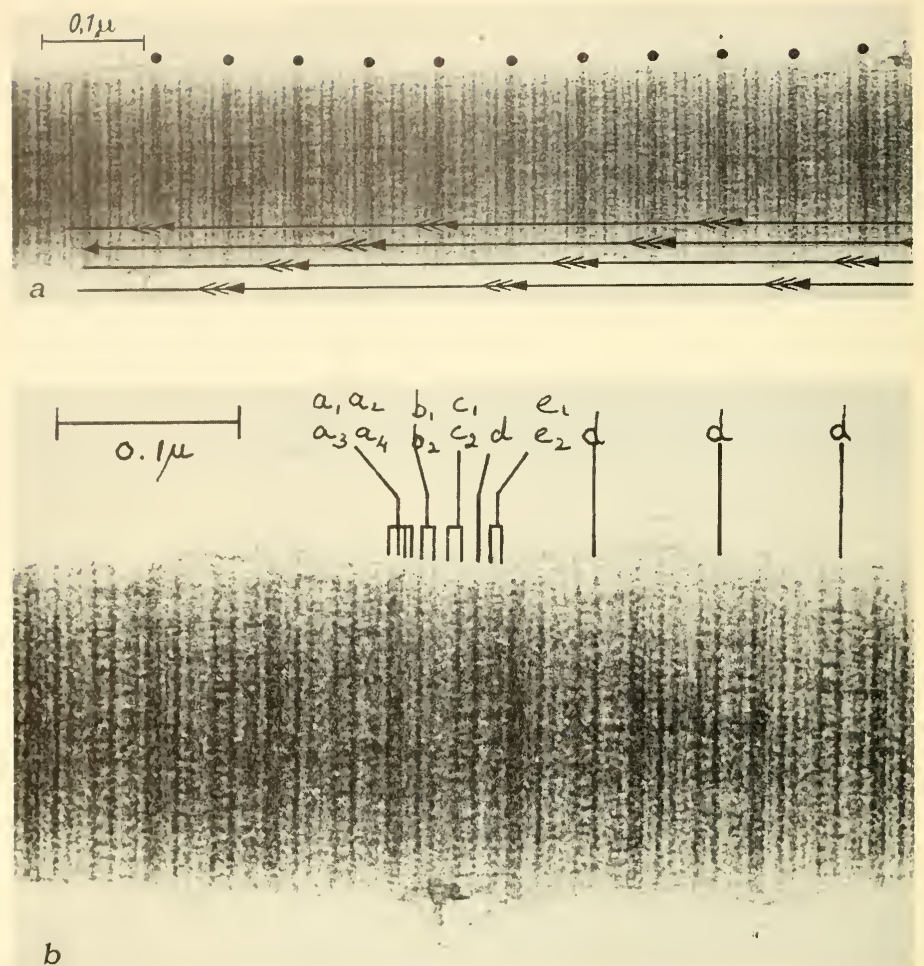

Fig. 7. Reconstituted native-type fibril, showing the characteristically polarized intraperiod band pattern. The axial period of ca. $700 \AA$ is indicated by the row of dots, and the longitudinally staggered packing of the protofibrils by the arrangement of arrows along the lower edge of the figure. (a) Stained with PTA. $\times 130,000$. (From Hodge, 1959b.) (b) Indicates the currently accepted band nomenclature. $\times 230,000$.

bands observable along the length of the TC, but that many of these bands differ markedly in relative intensities in the two fingerprints. Hence, we appear to have direct evidence that polar groups, both basic and acidic, are localized in narrowly defined regions at distinct and characteristic loci along the length of the TC macromolecules. The narrowest bands observed are in the range 15-20 $\AA$ wide. The intensity differences observed also indicate that while basic groups 
predominate in some of these loci, there is a relative parity or an excess of acidic groups in other regions. The analysis of the detailed band pattern of SLS enables one to determine in principle, at least, the packing arrangement of the TC macromolecules in any of the other ordered aggregation states.

If an acid solution of collagen is dialyzed against distilled water, the final result is the production of a water-clear gel, consisting of

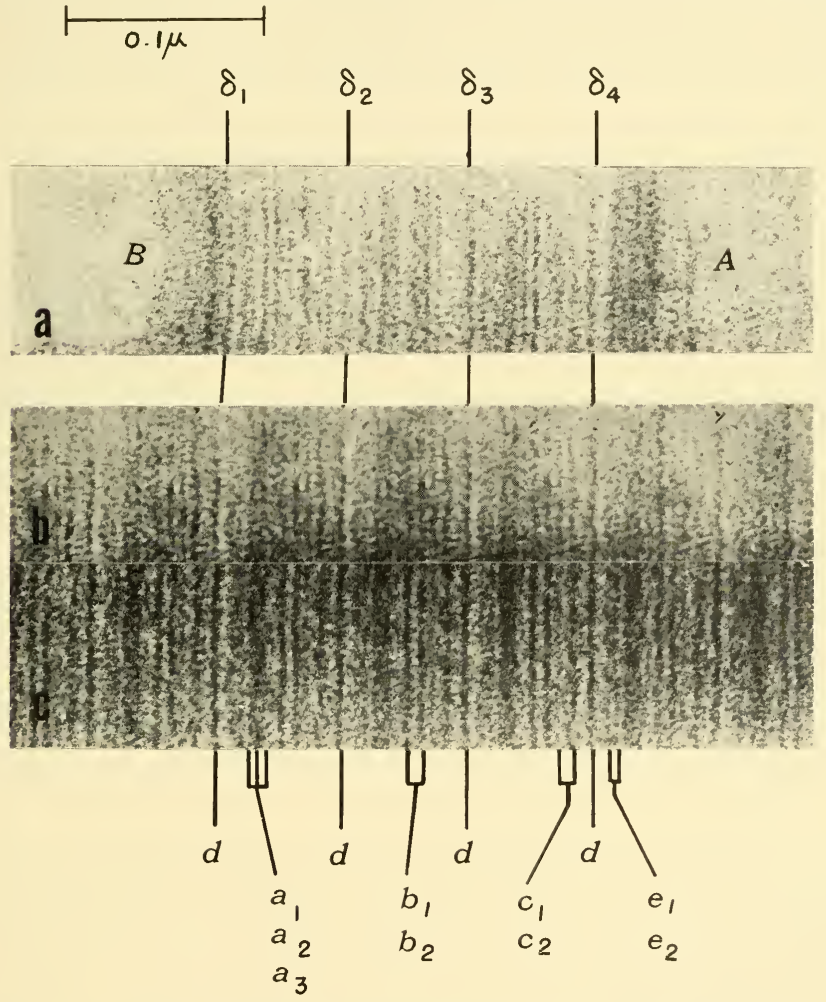

Fig. 8. Composite micrographs illustrating the optical synthesis (b) of the native-type band pattern, produced by multiple printing of the image of the single segment shown in (a), stained with PTA. The recording plate was moved a distarice corresponding to $1 / 4$ of the segment length in the axial direction prior to each consecutive exposure. The summation of band densities resulting from this overlap (b) corresponds closely to the band pattern of native-type fibrils (c). $\times 250,000$. (From Hodge and Schmitt, 1960.) 


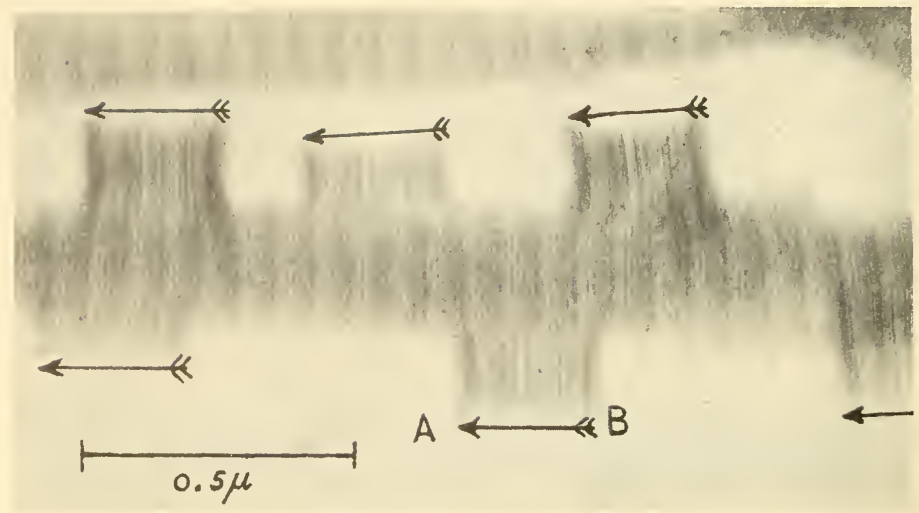

Fig. 9. Dimorphic ordered aggregate of TC, produced by exposing nativetype fibrils to a solution of TC containing ATP, under closely controlled conditions of $\mathrm{pH}$ and ionic strength. Note that all of the segments so formed are similarly polarized, as indicated by the labeled arrows, and are located identically with respect to the intraperiod band structure of the native-type fibril, which is itself polarized. Stained with PTA. $\times 70,000$. (From Hodge and Schmitt, 1960.)

very fine filaments with no striation visible in the electron microscope. The viscosity of the collagen solutions during such a dialysis rises steadily as the $\mathrm{pH}$ rises (Hodge and Schmitt, 1960; Hodge et al., 1960), indicating that end-to-end polymerization of the TC is giving rise to linear polymers or protofibrils. However, Glimcher and Bonar (personal communication) have shown that the smallangle $\mathrm{x}$-ray diffraction pattern of fibers drawn from such gels is characteristic of the native (700 A-repeat) type of packing of the TC. This means that the protofibrils are packing in staggered array (see Fig. 7) to form the very thin fibrils of the gel. If the dialysis is carried out in the presence of suitable concentrations of certain substances such as serum glycoprotein (see Schmitt et al., 1955 ), the TC comes out of solution in a second ordered fibrous form, the so-called fibrous long-spacing (FLS) form (Fig. 5). Since the axial period of FLS is about equal to the length of the TC macromolecule and the distribution of bands within each period is centrosymmetric, it seems likely that this type of structure arises by side-to-side aggregation of protofibrils in antiparallel array, i.e., with no preferred "polarity" (Figs. 1,5). This change in the inter- 


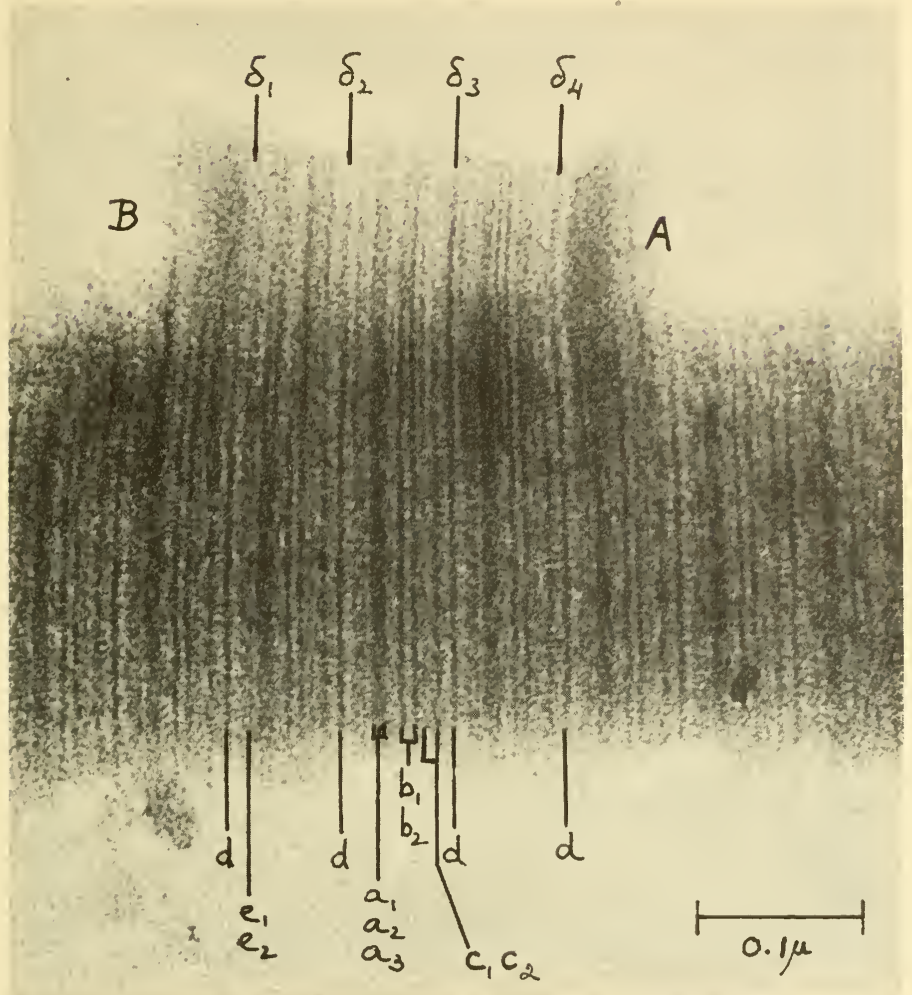

Fig. 10. Higher magnification view of a region in the same preparation as shown in Fig. 9, to illustrate the precise correspondence of the bands in the SLS-type structure with those in the native-type structure. As indicated in the band nomenclature (proposed by Hodge and Schmitt, 1960), each band in the native-type structure exhibits continuity with four "equivalent bands" of differing intensity in the SLS-type structure, and its intensity is therefore a function of the intensities of all four of the corresponding SLS bands. Thus, for example, $I_{d}=(k / 4)\left(I_{\delta_{1}}+I_{\delta_{2}}+I_{\delta_{3}}+I_{\delta_{4}}\right)$, where $I_{d}$ is the intensity of the $d$ band in the native-type structure, $1 \delta_{1}, 1 \delta_{2}, 1 \delta_{\delta_{3}}$, and $1 \delta_{4}$ are the intensities of the corresponding "equivalent bands" in the SLS-type structure, and $k$ is a constant, the value of which will depend on the degree of additivity of the band intensities. The close correspondence in the relative band intensities obtained by direct optical synthesis with those in the native-type fibril (Fig. 8) indicates that the actual value of $k$ must be close to unity, as would be expected if the staining intensity is proportional only to the concentration of polar side-chain groups. $\times 215,000$. (From Hodge and Schmitt, 1960.) 


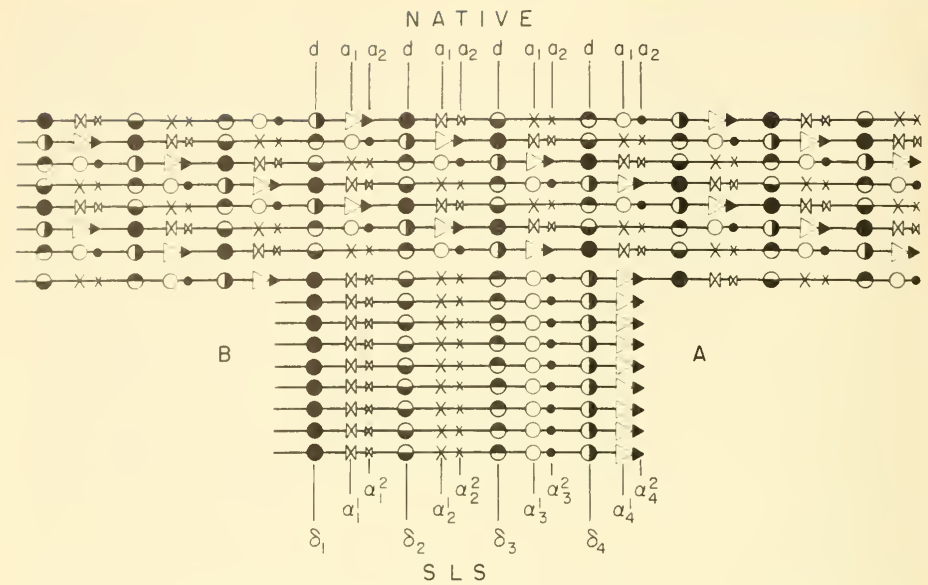

Fig. 11. Diagrammatic interpretation of the packing arrangements of the TC macromolecules in the dimorphic ordered forms shown in Figs. 9 and 10. To minimize complexity, only three of the twelve or so bands actually observable in the native-type structure are depicted. Note that in both the SLS-type and native-type structures, all staining loci are in accurate transverse alignment, i.e., in register. However, in the SLS-type, only like features are in register, i.e., "homo-register," while in the native-type structure, all bands arise by alignment of the four corresponding "equivalent loci" of the TC macromolecule, i.e., "hetero-register." In the SLS band terminology proposed by Hodge and Schmitt (1960), the superscripts refer to the subscripts in the generally accepted band nomenclature for the native-type fibril, the subscripts to the four corresponding "equivalent loci" in the TC macromolecule. Thus, for example, the $a_{1}$ band of the native-type fibril arises by juxtaposition of the $\alpha^{1}{ }_{1}, \alpha^{1} \Perp_{2}, \alpha^{1}{ }_{3}$, and $\alpha^{1}{ }_{4}$ sites of the TC macromolecule when they are placed in staggered array, obeying the selection rule that adjacent protofibrils are displaced relative to one another by a distance equal to $1 / 4$ of the length of the TC macromolecule in the axial direction. (From Hodge and Schmitt, 1960.)

action properties of the TC polymers is presumably the result of an altered charge distribution following binding of the strongly negatively charged glycoprotein at specific points of the TC. The presence of one exceptionally broad and dense band per period, and relatively few and rather diffuse intraperiod bands, suggests that most of the glycoprotein is adsorbed near the A and B ends of the TC. This would be in accord with the relatively high concentration of basic groups present in these regions as deduced from the appearance of PTA-stained segments (Figs. 2, 3). 

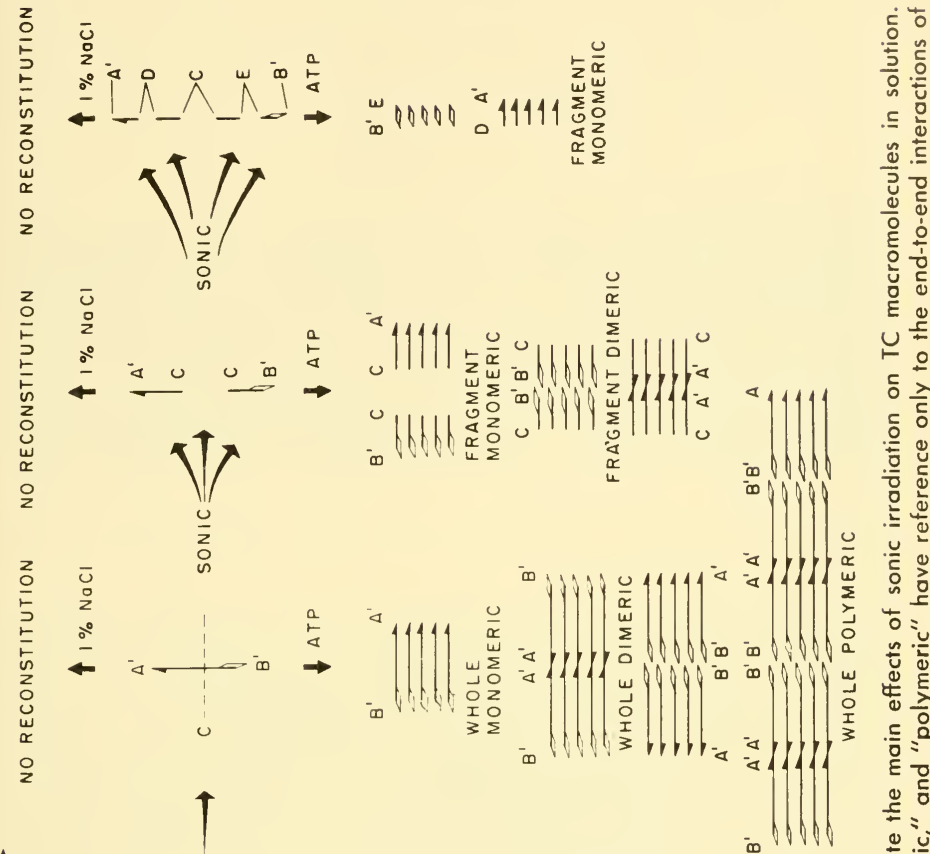

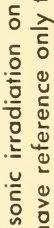
is 는 षे 悉交言 $\therefore$ : E ๘ $\neq$ :

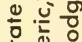
노 $\stackrel{5}{2}$

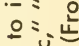
등 능 익 ํㅡㅇ 응 है i $=$ है ปั कृष 돋 
If the water dialysis is carried out with lower concentrations of the glycoprotein, a mixture of FLS and native-type ${ }^{\top}$ fibrils is obtained (Highberger et al., 1951). At still lower concentrations, only native-type fibrils are obtained. The system glycoprotein-collagen thus nicely illustrates the manner in which binding of a second component modifies the charge distribution at a given $\mathrm{pH}$ and ionic strength and changes the type of ordered interaction favored.

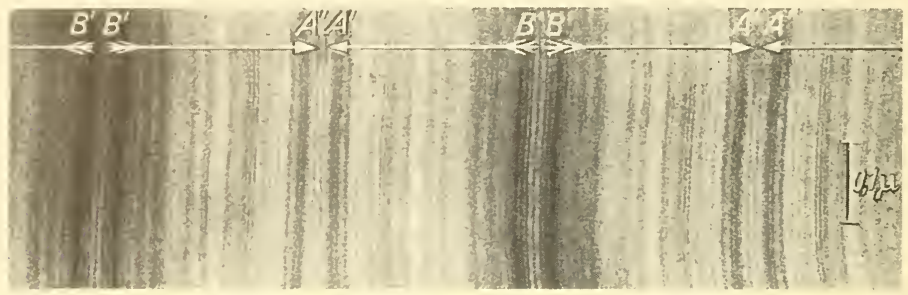

Fig. 13. SLS-type aggregate of whole polymeric type from a solution sonically irradiated $240 \mathrm{~min}$, showing end-to-end linkages of the type $A^{\prime}-A^{\prime}$ and $B^{\prime}-B^{\prime}$. Stained with PTA. $\times 100,000$. (From Hodge, 1959b.)

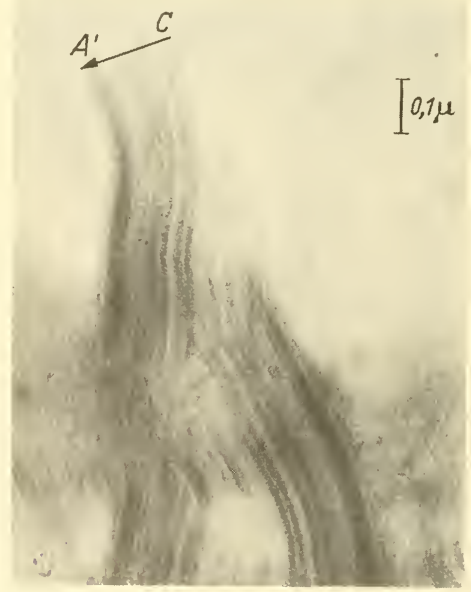

Fig. 14. SLS aggregates from a solution sonically irradiated $240 \mathrm{~min}$, showing formation of ribbon-like fragment monomeric forms of the type $A^{\prime} C$. Stained with PTA. $\times 68,000$. (From Hodge and Schmitt, 1958.)

${ }^{7}$ The term "native-type" refers to the normal aggregation state of TC in vivo, i.e., with an axial period of $c a .700 \mathrm{~A}$. 
Under other conditions, and particularly in the presence of ATP, the charge distribution on the protofibrils is such as to favor a "polymeric segment" type of structure, i.e., one in which all like features of the TC are in register across the fibril (Hodge and Schmitt, 1960). In this type of structure (Figs. 6, 23), which we have termed "fibrous-type segment long-spacing" (F-SLS) to distinguish it from the antiparallel FLS just described, the axial period is equal to the length of the TC macromolecule, and the polarized band pattern is equivalent to that obtained by placing single segments end-to-end in the sense $\mathrm{AB}-\mathrm{AB}-\mathrm{AB}$-. If suitably oriented fibers can be obtained from such preparations, it should be possible to obtain direct evidence concerning the precise distribution of density along the TC macromolecule by means of small-angle x-ray diffraction. Such data cannot be obtained by $x$-ray analysis of native-type collagen fibers, in which the TC macromolecules are packed in a staggered fashion with respect to their neighbors.

The ordered aggregation form of TC that is stable under physiological conditions and that occurs in various connective tissues will

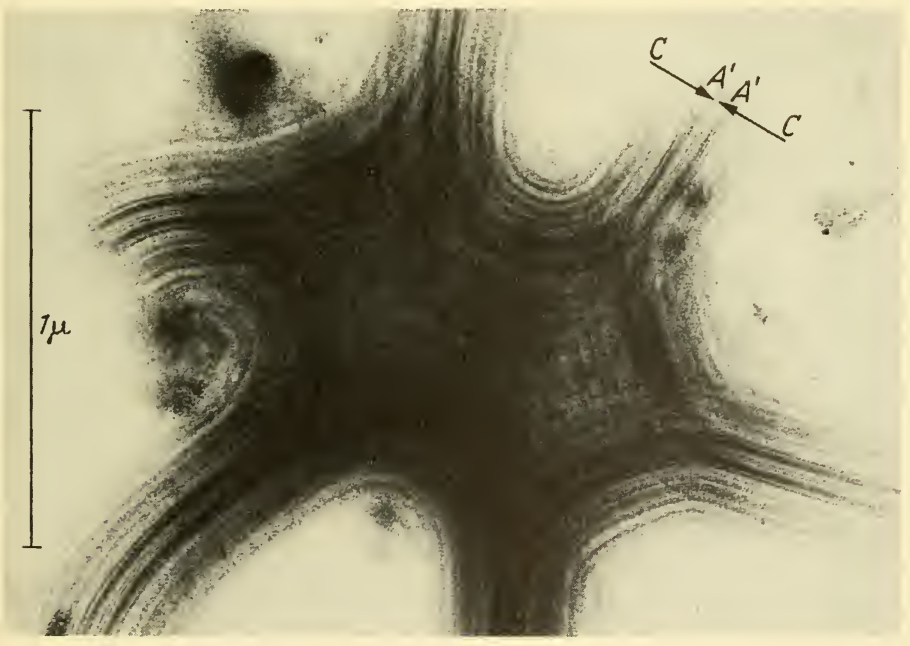

Fig. 15. SLS aggregates of the fragment dimeric type $C A^{\prime}-A^{\prime} C$ from a solution sonically irradiated $240 \mathrm{~min}$, showing extensive lateral aggregation in the form of "ribbons." Stained with PTA. $\times 55,000$. (From Hodge and Schmitt, 1958.) 


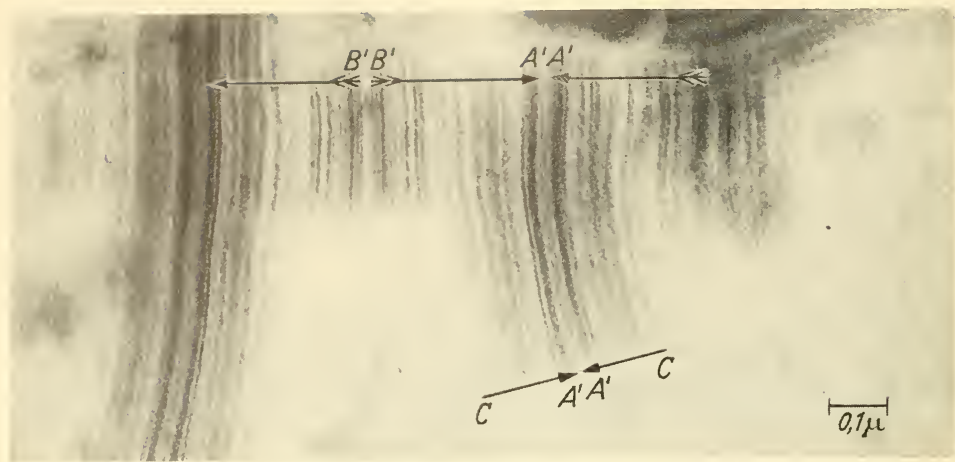

Fig. 16. (See next page for caption.)

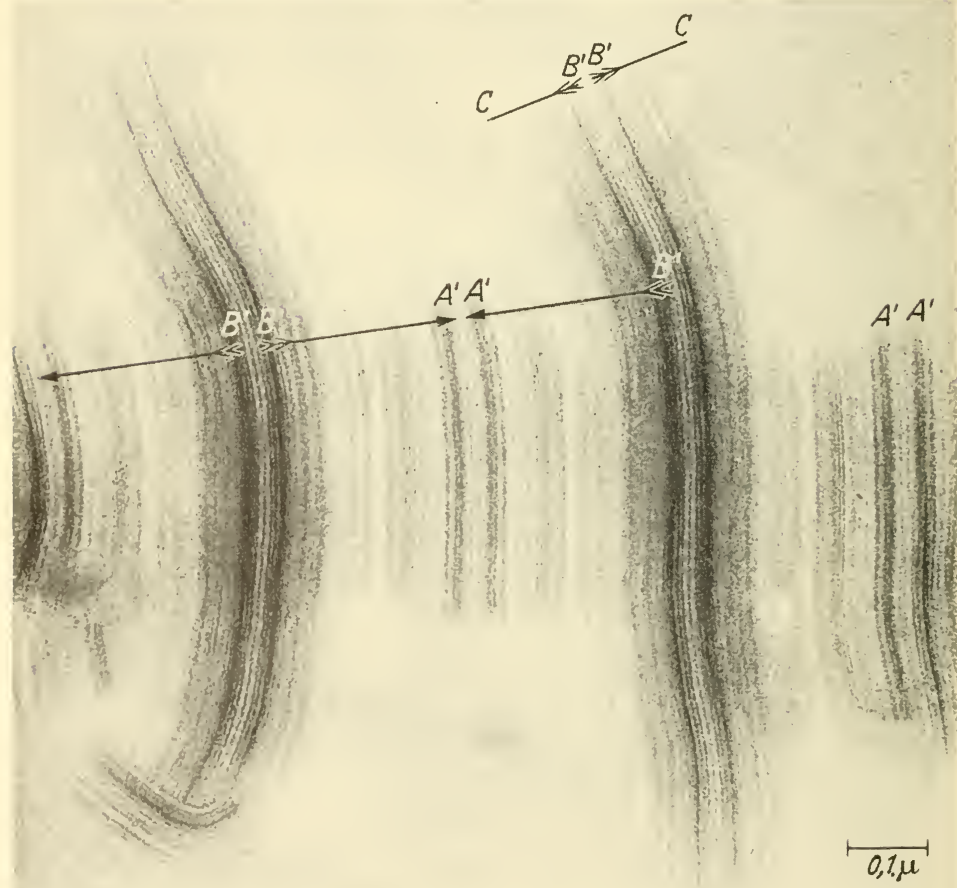

Fig. 17. (See next page for caption.) 
be referred to as the native type. This form may be reconstituted from solutions of TC under appropriate conditions (see Schmitt et al., 1955), has a fundamental axial period of about $700 \AA$, i.e., one-fourth the length of the TC macromolecule, and the intraperiod band distribution observed after electron staining is characteristically asymmetric or "polarized" (Figs. 1, 7). As indicated diagrammatically in these figures, the formation of native-type collagen fibrils involves packing of protofibrils (linear polymers of TC) so that neighboring protofibrils are displaced longitudinally in relation to one another by one-fourth of the TC length. That this must be the case is readily apparent from the facts that (a) the axial period of native-type collagen is one-fourth that of the length of individual macromolecules and (b) the additive sum of any asymmetric density distribution of length $L$ displaced longitudinally and consecutively by a distance $L / p$, where $p$ is an integer, results in the generation of an asymmetric density function of like "polarity" and having an axial period of $L / p$. For native-type collagen, $p=4$. It has been shown that other values of $p$ pertain to other fibrous proteins, e.g., paramyosin (Hodge, 1959a). In principle, it should be possible to reconstruct the salient features of the band pattern of native collagen by a process of optical synthesis from the band pattern of SLS, and, in fact, such syntheses have been carried out in this laboratory (Fig. 8), with resolution limited only by the uncertainties in the absolute positions of the various SLS bands introduced by disordering during drying of the specimens for examination in the electron microscope.

However, a more definitive proof of the correctness of the stagger theory has been forthcoming in recent work (Hodge and Schmitt, $1960)$. In these experiments, fibrils of native type (700 $\AA$-repeat) were placed in an environment containing monomeric TC under conditions favoring the formation of SLS-type aggregates. It was found that the TC macromolecules in the surfaces of the native-type fibrils had apparently acted as "nuclei" for the growth of individual segments (Fig. 9). In the resultant dimorphic ordered forms, which

Fig. 16. Whole polymeric SLS form of sonically irradiated calfskin collagen, showing isomorphous growth of fragment dimeric ribbons of the type $C A^{\prime}-A^{\prime} C$. Stained with PTA. $\times 74,000$. (From Hodge and Schmitt, 1958.)

Fig. 17. Whole polymeric SLS form of sonically irradiated calfskin collagen, showing isomorphous growth of fragment dimeric ribbons of the type $C B^{\prime}-B^{\prime}$ C. Stained with PTA. $\times 100,000$. (From Hodge, 1959b.) 
allow a direct comparison of the band patterns of both the segment and native forms, it is observed that each band in the native-type fibril exhibits a direct transverse continuity with one of four different bands of the segment pattern (Figs. 10, 11), each of different intensity and separated longitudinally from one another by $L / 4$, where $L$ is the length of the TC macromolecule. It is clear, therefore, that the density observed in any particular band of the nativetype structure represents a summation of the densities of the appropriate four "equivalent bands" in the segment-type structure. It should be noted that, although the bands observed in SLS, whether stained for basic or acidic side-chains, obey this selection rule (i.e., may be classified in groups of four "equivalent bands" spaced $L / 4$ apart), this does not imply that the TC macromolecule consists of four identical subunits. In any case, the physical properties of collagen solutions and the distribution of density in the

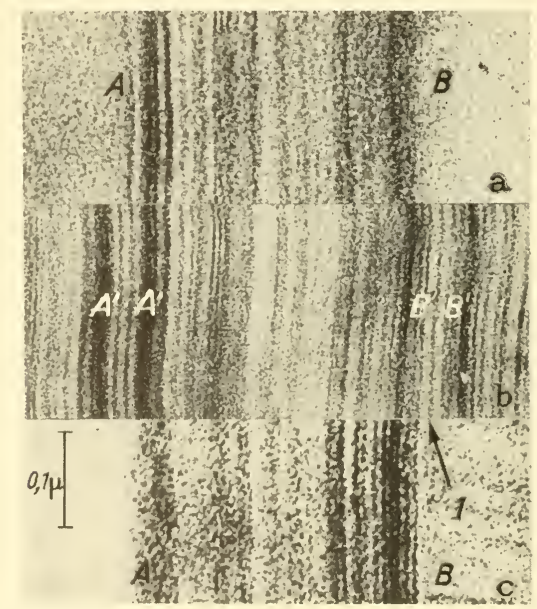

Fig. 18. Composite micrograph for comparison of the band patterns of whole monomeric forms (single segments, a and c) with that of the whole polymeric form (b). The use of two single segments is necessary since the band pattern is often obscured at one or both ends by disorder resulting from drying of the specimen. Arrow 1 indicates the new band observed in the $B^{\prime}-B^{\prime}$ junctions of polymeric SLS forms, which is not present in single segments, and apparently is the result of end-to-end polymerization of the TC macromolecules. Calfskin collagen. Stained with PTA. $\times 120,000$. (From Hodge and Schmitt, 1958.) 
SLS band pattern suffice to prove that the $2800-\AA$ macromolecular unit is the true monomeric form.

Electron microscope examination of SLS-type aggregates obtained from solutions of TC subjected to sonic irradiation has served to reveal some features of interest, particularly in relation to the end-to-end polymerization properties of TC. The physicochemical measurements of Nishihara and Doty (1958) indicated that sonic irradiation fragmented the TC macromolecules into shorter pieces which, however, largely retained the helical configuration of the native macromolecules. They concluded that the time-dependence of the molecular weight change was compatible with a preferential fragmentation of the macromolecules into halves and quarters. Electron microscope examination of SLS-type aggregates produced from such sonicated solutions confirmed this conclusion in general (Hodge and Schmitt, 1958) and, in addition, showed that sonic irradiation profoundly modifies the end-to-end interactions of the TC.

The main effects of sonic irradiation on the TC macromolecule are illustrated diagrammatically in Fig. 12. One of the most important is a rapid alteration of "end-regions" without apparent change in length, the modification being manifested as a change in the ability of the TC to engage in end-to-end polymerization. This is indicated in Fig. 12 by labeling the ends $A^{\prime}$ and $B^{\prime}$ rather than A and B. An important consequence of this modification is an impairment of the ability to form fibrils of native type, i.e., an inhibition of end-to-end polymerization of the type $\mathrm{AB}-\mathrm{AB}-\mathrm{AB}-$ involved in the protofibril formation. Another result of sonic irradiation is a pronounced effect on the characteristics of the SLS-type precipitates formed on addition of ATP. The SLS-type precipitates derived from unirradiated control solutions usually consist largely of single segments with relatively few dimeric or trimeric forms, the latter normally exhibiting end-to-end linkages of type A-B. However, the irradiated solutions yield progressively increasing amounts of dimeric and polymeric forms (see Fig. 12 for explanation), involving end-to-end linkages of the type $A^{\prime}-A^{\prime}$ and $B^{\prime}-B^{\prime}$ (Fig. 13). Thus, in addition to fragmentation, the effects of sonication are apparently twofold: (a) impairment of end-to-end interactions of the A-B type under conditions which normally favor the formation of protofibrils and fibrils of native type and (b) enhancement of endto-end linkages of type $A^{\prime}-A^{\prime}$ and $B^{\prime}-B^{\prime}$ under circumstances which 
(19)

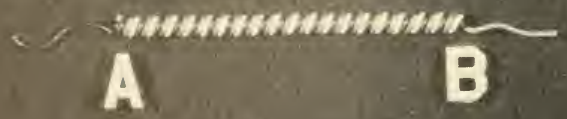

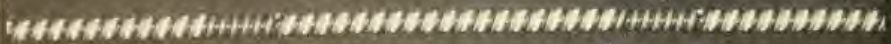
B A
B A

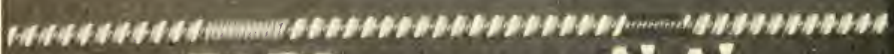

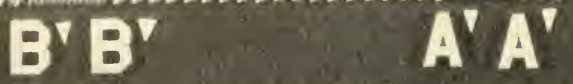

Fig. 19. (See next page for caption.)

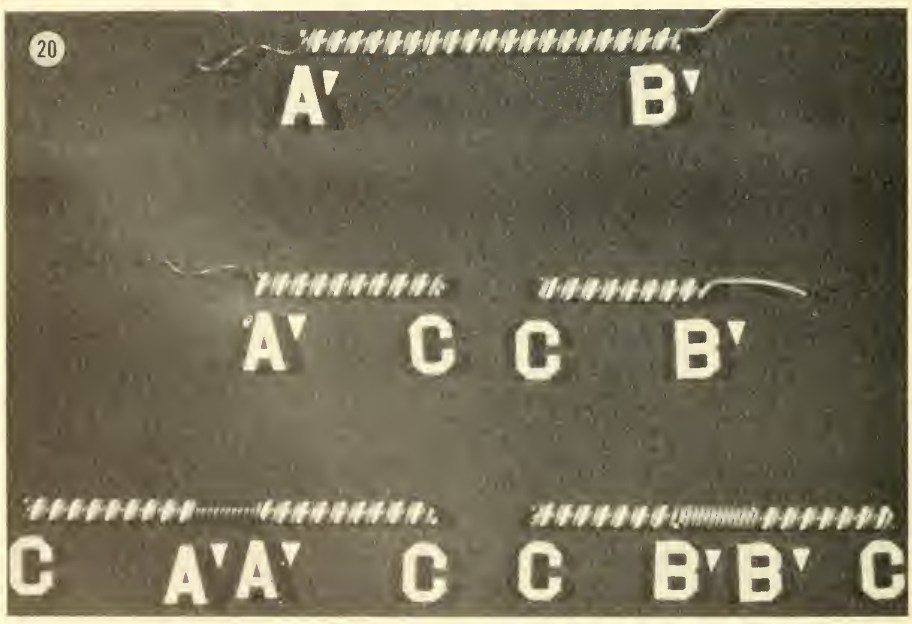

Fig. 20. (See next paqe for caption.) 
usually yield single segments (whole monomeric type) together with some polymeric forms of type A-B.

With longer times of irradiation, scission of the macromolecules becomes evident in SLS-type precipitates (Figs. 14-17). Fragmentation appears to occur initially near the middle of the TC macromolecule, yielding fragments $\mathrm{A}^{\prime} \mathrm{C}$ and $\mathrm{B}^{\prime} \mathrm{C}$ about 55 per cent and 45 per cent, respectively, of the original TC length. These are capable of forming monomeric (Fig. 14) and dimeric (Fig. 15) SLStype aggregates, and of isomorphous growth on whole polymeric SLS (Figs. 16, 17). However, dimerization or polymerization of the scission products apparently never involves new ends resulting from fragmentation; i.e., end-to-end linkages of the type C-C have not so far been observed. It would appear, therefore, that the original ends of the TC macromolecules have properties which are not duplicated in the new ends formed by scission. Extensive irradiation results in still smaller fragments of the type indicated by breakage at points D and E in Fig. 12. Of the four scission products presumably yielded by this process, only those which include $\mathrm{A}^{\prime}$ or $\mathrm{B}^{\prime}$ ends have been observed to form ordered SLS-type structures.

Detailed comparison of the band pattern of single segments (whole monomeric SLS form) with that found in the whole polymeric form resulting from sonic irradiation has led to a hypothesis concerning the mechanism by which TC macromolecules engage in end-to-end linkage to form protofibrils (Hodge and Schmitt,

Fig. 19. At top is depicted a model of the TC macromolecule in solution, the three polypeptide chains being represented by wires (black, gray, and white, respectively). Within the body of the macromolecule, the three wires are coiled about one another in an orderly helical pattern, representing the type of structure deduced from large-angle $x$-ray diffraction studies of collagen. At the ends, the postulated end-chains are shown as single, randomly coiled, polypeptide chains. Note that parameters, such as the length and diameter of the macromolecule, the pitch of the helix, and the lengths of the end-chains, are not to scale. In the center is illustrated the heterologous type of end-to-end coiling envisaged in the formation of protofibrils during normal polymerization (end-to-end linkage of type A-B), while at the bottom is shown the homologous type of end-chain coiling presumed to occur during formation of whole polymeric SLS-type aggregates, such as those shown in Figs. 13, 16, 17. (From Hodge, 1959b.)

Fig. 20. The same model of the TC macromolecule as shown in Fig. 19, illustrating the fragmentation by sonic irradiation (center), and the formation of fragment dimeric forms $C A^{\prime}-A^{\prime} C$ and $C B^{\prime}-B^{\prime} C$ (at bottom) on the addition of ATP. Dimeric and polymeric forms involving junctions of type $C-C$ have not been observed. (From Hodge, 1959b.) 
1958). In the $A^{\prime}-A^{\prime}$ junction (Fig. 18) of the polymeric form, the two bands corresponding to the A-terminal band of the monomeric SLS form appear as a doublet separated by an interband region about $100 \AA$ wide. Similarly, in the $B^{\prime}-B^{\prime}$ junction, the two B-terminal bands are spaced about $180 \AA$ apart, and a new band (arrow 1 in Fig. 18), not observed in monomeric SLS, is seen in the middle of the junctional regions. If it is assumed that well-defined bands, as discussed earlier, occur only in those regions where the polypeptide chains are in an orderly helical configuration, thus providing the specific array of polar side-chains necessary for the cooperative binding of ions such as PTA, these observations suggest the following conclusions (see Figs. 19, 20):

1. The TC macromolecule, the bulk of which is presumably in the characteristic three-strand helical configuration deduced from x-ray diffraction studies, possesses specialized "end structures" which are most probably in the form of short dangling chain appendages with maximum lengths of about $100 \AA$ and $180 \AA$ for the A and B ends, respectively. Such terminal peptide chains would most likely adopt a random coil configuration in solution, as indicated diagrammatically in Figs. 19 and 20.

2. The appearance of a new band in the center of the $B^{\prime}-B^{\prime}$ junctions of whole polymeric SLS forms suggests that end-to-end polymerization of TC involves an orderly coiling of these terminal chains about one another to form a junctional region with an ordered helical structure, stabilized perhaps by hydrogen bonds. It should be noted that this is the simplest hypothesis. However, the possibility is not excluded that at one end there may be two polypeptide chains, in which case the formation of polymers involving linkages of the type $\mathrm{A}-\mathrm{B}$ would result in the formation of a three-stranded structure in the junctional regions. Such a structure would pose a problem in finding a structural basis for end-to-end interactions of homologous type $\left(\mathrm{A}^{\prime}-\mathrm{A}^{\prime}\right.$ or $\left.\mathrm{B}^{\prime}-\mathrm{B}^{\prime}\right)$.

On the basis of the picture just outlined, the presence of a new band in the $B^{\prime}-B^{\prime}$ junction and the absence of such a band in the $\mathrm{A}^{\prime}-\mathrm{A}^{\prime}$ junction are indicative of differences in amino acid composition, particularly of polar groups, between the A and B end-chains. Indeed, it seems likely that the specificities of the terminal chains reside in just such differences of amino acid composition and perhaps to some extent depend on the presence of other components such as carbohydrate. The marked changes in the end-to-end inter- 
action properties of TC resulting from sonic irradiation are presumably due to some modification of one or both of these specialized end-structures, possibly the splitting off of a peptide or other small fragment.

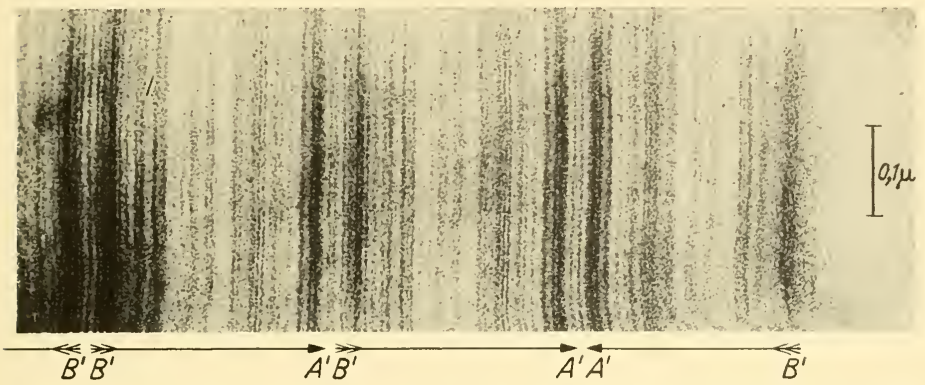

Fig. 21. Whole polymeric SLS form from a solution sonically irradiated $480 \mathrm{~min}$, showing the detailed band structure within an $A^{\prime}-B^{\prime}$ junction, together with the more commonly observed $A^{\prime}-A^{\prime}$ and $B^{\prime}-B^{\prime}$ junctions. Stained with PTA. $\times 120,000$. (From Hodge, 1959b.)

Occasional junctions of the type $A^{\prime}-B^{\prime}$ have been observed in polymeric SLS precipitates obtained from sonicated solutions of TC (Fig. 21), and their band structure agrees well with that to be expected from an analysis of $\mathrm{A}^{\prime}-\mathrm{A}^{\prime}$ and $\mathrm{B}^{\prime}-\mathrm{B}^{\prime}$ junctions. The $\mathrm{A}^{\prime}-\mathrm{B}^{\prime}$ junctions have also proved useful in the detailed analysis of the native-type ( $700 \AA$-repeat) band pattern in terms of the SLS pattern.

\section{Effects of Proteases on Tropocollagen}

The effects of proteolytic enzymes such as trypsin, chymotrypsin, and pepsin on the polymerization properties of tropocollagen macromolecules have been investigated in a recent series of experiments (Hodge et al., 1960). The results to date have been entirely consistent with the end-chain hypothesis already described.

The data indicate that many solutions of "soluble collagen" are relatively heterodisperse in that they contain variable amounts of linear dimers, trimers, and higher polymers in addition to large numbers of monomeric TC macromolecules. As indicated by the viscosity data mentioned earlier, the proportion of polymers increases as the $\mathrm{pH}$ is raised on the acid side of the isoelectric point. The gross macroscopic appearance of the precipitates obtained on 


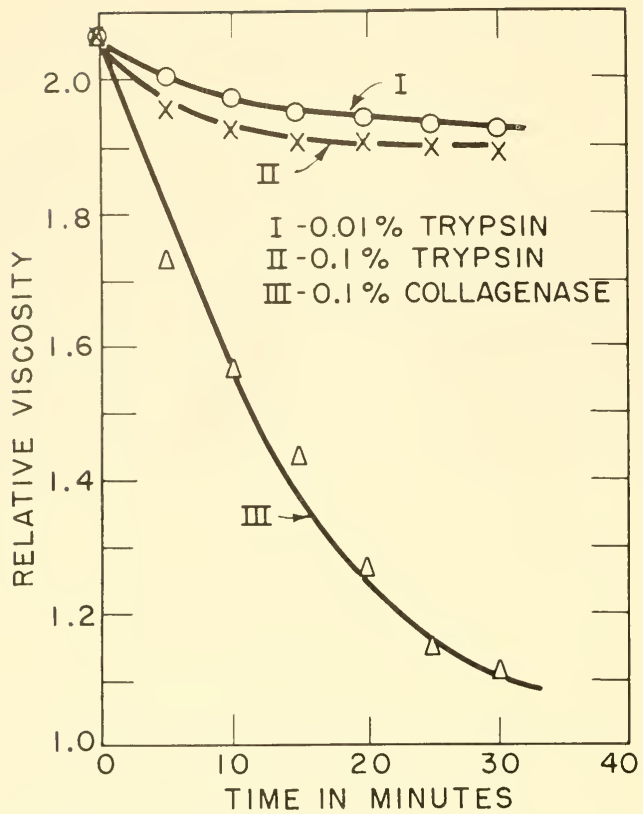

Fig. 22. Graph to illustrate the effect of two proteolytic enzymes (trypsin, Worthington, $2 \times$ recrystallized, and collagenase, Worthington) on the viscosity of a typical solution of calfskin collagen in tris buffer, $\mathrm{pH} \mathrm{6.9,} \mathrm{in} \mathrm{the} \mathrm{presence}$ of $0.5 \mathrm{M} \mathrm{CaCl}, 2$ at $20^{\circ} \mathrm{C}$. The enzymes were dissolved in the buffer solution and the zero points obtained by measuring the viscosity of an aliquot of the collagen solution when diluted with a volume of buffer equal to that used in adding the enzyme. Note that while collagenase rapidly reduces the viscosity to a low value, the effect of trypsin is a small but rapid reduction of the viscosity to a high steady value and appears to be dependent on the enzyme concentration.

addition of ATP is apparently a reliable criterion of the degree of homogeneity of such solutions; when appreciable amounts of linear polymers are present, addition of ATP produces precipitates which are of a flocculent, fibrous character and do not settle even on prolonged standing. These macroscopic characteristics are supported by electron microscope observations which consistently show "strings" of segments, apparently arising from the "crystallization" of TC macromolecules on the linear polymers present in the solution. On the other hand, the addition of ATP to acid collagen solu- 


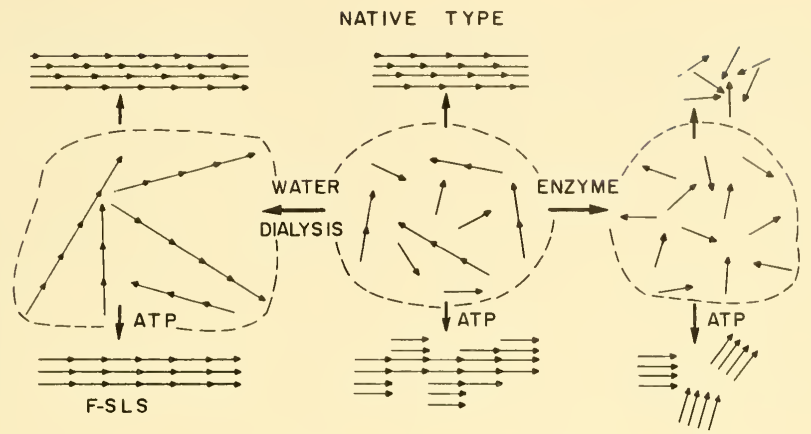

Fig. 23. In the center is depicted a typical acid solution of collagen, containing TC monomers together with some linear dimers, trimers, and other $n$-mers. Under suitable conditions (e.g., dialysis against $\mathbf{i}$ per cent $\mathrm{NaCl}$ ), the solution yields native-type fibrils (top center), while the addition of ATP gives rise to a polymeric SLS-type precipitate resulting from the growth of individual segments on the pre-existing linear polymers of TC. At left is shown the linear polymerization resulting from dialysis against distilled water, ultimately leading to the formation of a water-clear gel consisting of very thin fibrils of native type (top left). The addition of ATP during late stages of the dialysis leads to the formation of polymeric SLS-type structures (F-SLS at bottom left). At right is illustrated the depolymerizing action of proteases such as pepsin (see Fig. 22). Subsequent dialysis against distilled water does not induce end-to-end polymerization (right center and top right), and the addition of ATP produces only single segments, i.e., whole monomeric forms (bottom right).

tions after treatment with either trypsin or pepsin (at appropriate $\mathrm{pH}$ values) produces a non-fibrous precipitate, eventually settling to a dense mass at the bottom of the tube. When examined in the electron microscope, such preparations exhibit only single segments, with very few dimeric or higher polymeric forms. It would seem, therefore, that a hitherto unrecognized but important action of these proteolytic enzymes on collagen in solution is a depolymerization of protofibrils without apparent impairment of the three-stranded helical structure of the individual macromolecules. This interpretation is supported by observations on the physical properties of collagen solutions during attack by pepsin or trypsin. On exposure to pepsin or trypsin (under suitable conditions of $\mathrm{pH}$, temperature, and ionic strength), the viscosity of collagen solutions undergoes a small'but rapid decrease (see also Gross, 1958) to a still high value consistent with the presence of TC monomers in the solution (Fig. 22 ); the optical rotatory properties of the solution do not change 
appreciably during this process. In contrast to this, exposure to collagenases causes both the optical rotation and the viscosity to decrease rapidly (Seifter et al., 1958).

These results are compatible with the hypothesis that the enzymatic attack of pepsin and trypsin on collagen is confined to a limited region at the ends of the TC macromolecule, yielding modified macromolecules whose length and internal helical configuration remain essentially unaltered, but whose end-to-end polymerization properties have been drastically modified (Fig. 23). The enzymetreated solutions yield single segments (whole monomeric type) which are normal as judged by their band pattern on staining with PTA. In contrast to this, the same collagen solutions fail to give ordered aggregates of segment type after treatment with collagenase. Treatment with pepsin or trypsin also prevents the formation of a water-clear gel on dialysis against distilled water, and almost

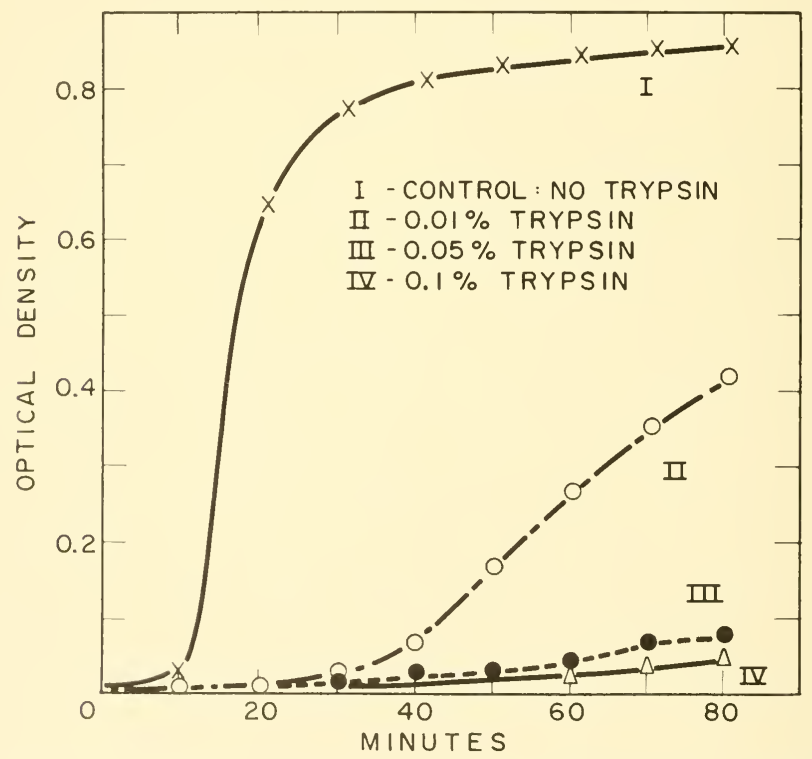

Fig. 24. The effect of treatment with trypsin (Worthington, $2 \times$ recrystallized) on the thermal gelation of a 0.1 per cent solution of calfskin collagen (tris buffer, $\mathrm{pH} \mathrm{7,} \mathrm{with} 0.45 \mathrm{M} \mathrm{NaCl}$ ) at $34^{\circ} \mathrm{C}$, after incubation with the enzyme $6 \mathrm{hr}$ at $20^{\circ} \mathrm{C}$, as determined by the optical density at $430 \mathrm{~m} \mu$. 
completely eliminates the production of native-type fibrils on dialysis against 1 per cent $\mathrm{NaCl}$. The phenomenon of "thermal gelation" of neutral salt solutions of collagen investigated extensively by Gross (1956, 1959) and by Bensusan and Hoyt (1958) is also severely inhibited or abolished if the collagen solutions are incubated with trypsin at $20^{\circ} \mathrm{C}$ prior to incubation in the temperature range $30^{\circ}-37^{\circ} \mathrm{C}$. The effect of the enzyme is illustrated in Fig. 24. That the attack of pepsin and trypsin is largely, if not entirely, confined to the "end regions" of intact molecules is also suggested by the following result. When a sonicated solution of TC, which normally yielded a large proportion of whole polymeric and fragment dimeric SLS-type aggregates on addition of ATP (Figs. $13,15-17$ ), was treated with pepsin at $\mathrm{pH} 3-4$, prior to the addition of ATP, it yielded only whole and fragment monomeric SLS forms (Fig. 25).

It seemed likely from these data that the result of protease action on the TC macromolecule is the profound alteration, or more prob-

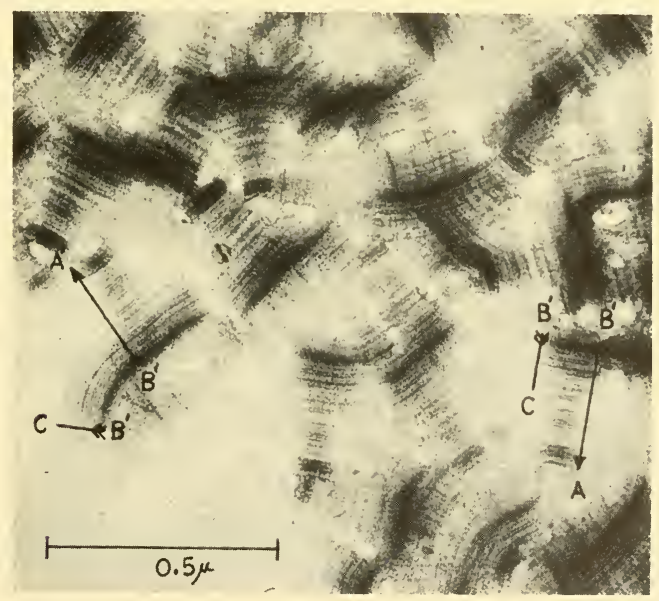

Fig. 25. A typical field in a preparation of SLS-type aggregates produced by addition of ATP to a solution of sonically irradiated collagen following incubation with pepsin. This solution, which, in the absence of enzyme treatment, characteristically yielded SLS-type structures of whole polymeric and fragment dimeric type (Figs. 13, 15-17), gave, after treatment with enzyme, only ordered aggregates corresponding to whole and fragment monomeric types, indicating that the enzyme inhibits end-to-end linkages of the TC macromolecules. Stained with PTA. $\times 90,000$. 
ably the splitting off, of one or more peptides of the end regions, perhaps one or both of the hypothetical "end-chains," or portions thereof. Efforts were therefore slanted toward the isolation and characterization of any such peptide products, utilizing chiefly the techniques of paper-curtain electrophoresis and two-dimensional paper chromatography. Preliminary results have been reported recently (Hodge et al., 1960). In evaluating possible methods for detecting and isolating products of protease action, we were guided by several considerations. In the first place, it seems to be generally accepted that the most highly purified collagens still contain a small

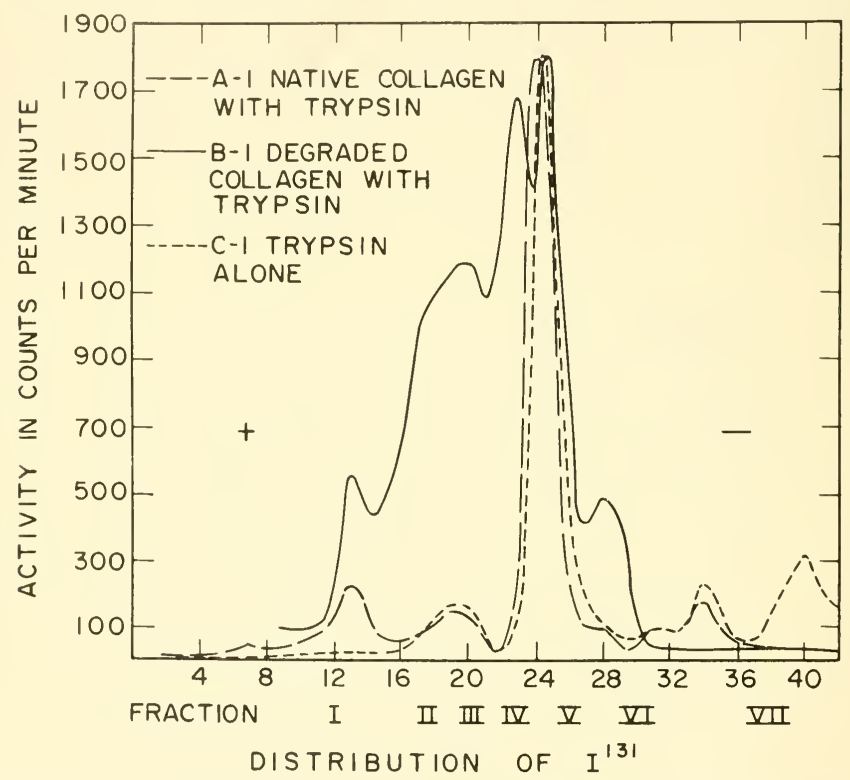

Fig. 26. The distribution of $\mathrm{I}^{131}$ activity in the effluent from paper-curtain electrophoresis at $\mathrm{pH} 4.9$ in pyridine-acetic acid buffer for $(\mathrm{A}-1)$ native calfskin collagen in solution at $\mathrm{pH} 7$ (tris buffer with $0.5 \mathrm{M} \mathrm{CaCl} \mathrm{C}_{2}$ ) incubated with trypsin (Worthington, $2 \times$ recrystallized) $2 \mathrm{hr}$ at $20^{\circ} \mathrm{C}$, followed by iodination; $(B-1)$ skin collagen after thermal denaturation and tryptic digestion following the procedure of Grassmann ef al. (1956), followed by iodination; and (C-1) trypsin alone (Worthington, $2 \times$ recrystallized) after incubation at $20^{\circ} \mathrm{C}$ in the same medium as used for Run A-1, followed by iodination. A control run with native collagen alone gave no significant activity in the region of Fraction I. It can be seen that the peak of activity labeled Fraction I on the acid side of the diagram appears to arise by the action of trypsin on both native and denatured TC. Input at center. (From Hodge et al., 1960.) 
but definite amount of tyrosine (Highberger, 1956), and the ability of the TC to form highly ordered fibrous structures is lost if the purification treatment is such that the tyrosine is removed. In themselves, these results suggest that the tyrosine is located in relatively accessible regions of the macromolecules, perhaps at the ends. This possibility was given further force by the observation of Bensusan (1959) that iodine markedly accelerates the thermal gelation of neutral salt solutions of collagen, especially in view of the fact that, with the exception of histidine which reacts much more slowly with
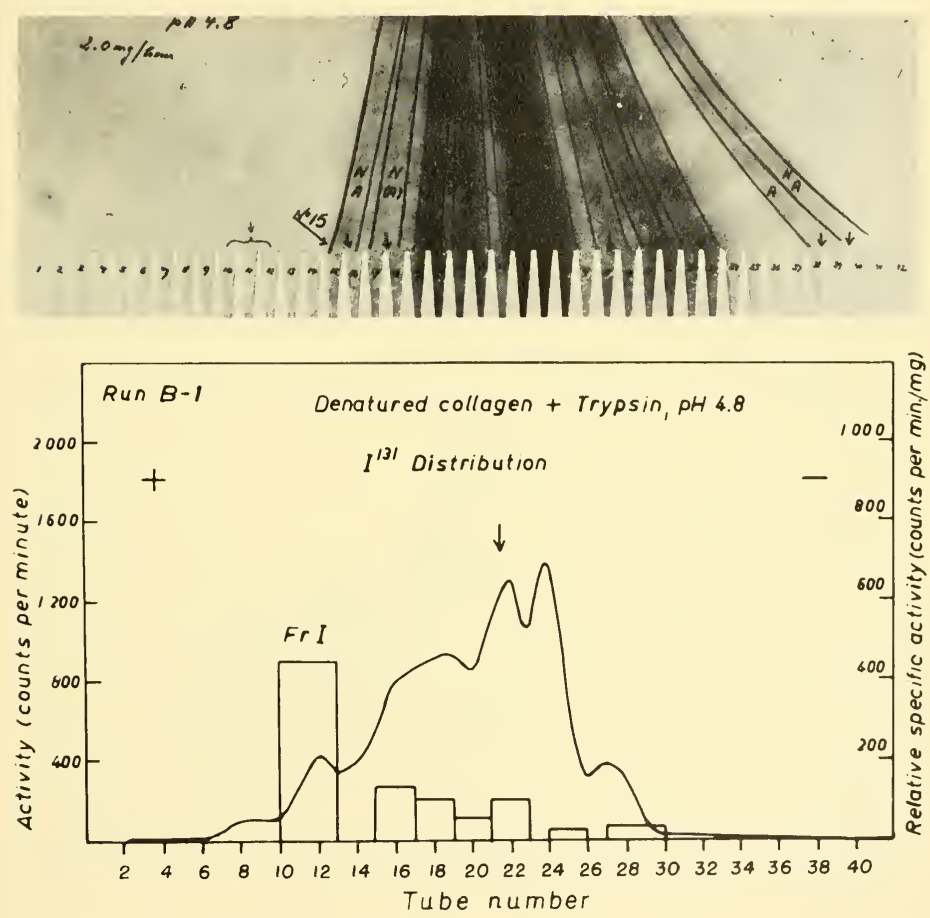

Fig. 27. The distribution of $\mathrm{I}^{131}$ activity in paper-curtain electrophoresis of denatured collagen incubated with trypsin (Run B-l of Fig. 26), together with the relative specific activities of the various fractions indicated in Fig. 26. At top is 'shown the electropherogram of the same run after staining, first with ninhydrin (positive reaction indicated by $N$ ), then with amido-black (A). Note that Fraction I escaped detection by staining with ninhydrin and amido-black, but has by far the highest specific activity of the various fractions examined. (From Hodge et al., 1960.) 
iodine, tyrosine is the only amino acid in collagen capable of iodination. A further pointer in this direction was the report by Grassmann et al. (1956) of a tyrosine-containing peptide, the $S_{\mathrm{f}}$ peptide, in tryptic digests of thermally denatured collagen. ${ }^{8}$

In view of the above considerations, it seemed desirable to follow the distribution of tyrosine in paper-curtain electrophoresis of trypsin-treated collagen by iodination of the digest with iodine containing the radioactive isotope $\mathrm{I}^{131}$. Figure 26 shows the distribution of radioactivity observed in electropherograms of a control run with trypsin alone, a run with trypsin and native (undenatured) collagen

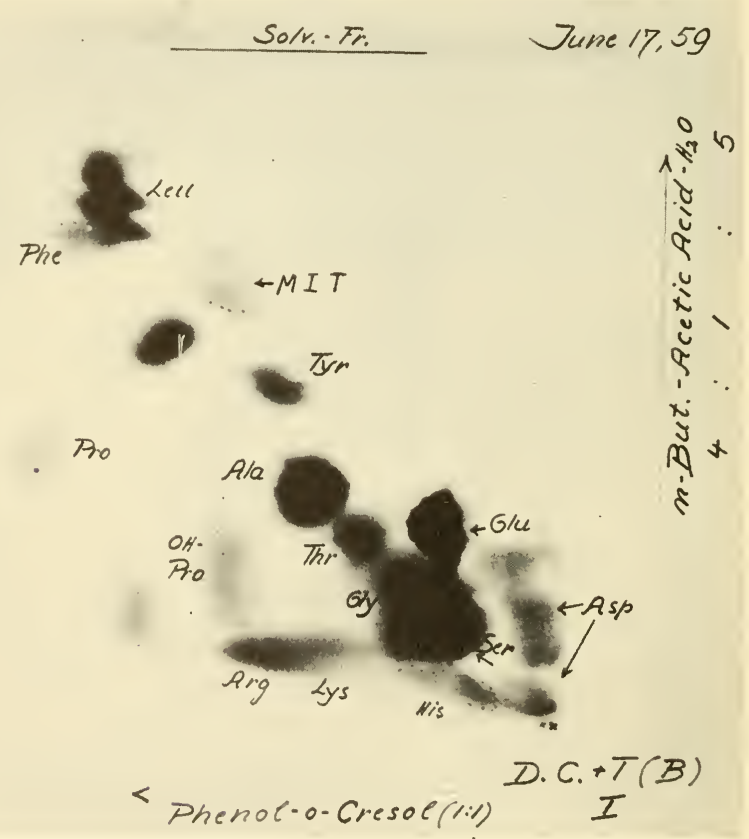

Fig. 28. Two-dimensional paper chromatogram of an acid hydrolysate of Fraction 1 from Run B-1 shown in Figs. 26, 27, run in phenol-o-cresol $(1: 1)$ and $n$-butanol-acetic acid- $\mathrm{H}_{2} \mathrm{O}$. Note the relatively large amounts of tyrosine (Tyr) and mono-iodo-fyrosine (MIT). (From Hodge et al., 1960.)

${ }^{8}$ Recent results of Grassmann (personal communication) indicate that the $S_{\mathrm{f}}$ peptide arises as a result of chymotrypsin activity (an impurity in the trypsin used in previous experiments) rather than from the activity of trypsin itself. 
in solution, and a run with trypsin and collagen denatured according to the methods described by Grassmann et al. (1956). Although the pattern is complex, it can be seen that for both the native collagen plus trypsin $(\mathrm{NC}+\mathrm{T})$ and denatured collagen plus trypsin $(\mathrm{DC}+\mathrm{T})$ runs an acidic peptide fraction (Fraction $\mathrm{I})$, not present in either of the control rums, was obtained. Figure 27 shows the electropherogram for the run with denatured collagen plus trypsin, together with the distribution of radioactivity and an indication of the relative specific activities ( $\mathrm{cpm} / \mathrm{mg}$ ) found for the various fractions. The high specific activity found for Fraction I indicated that this peptide fraction must be rich in tyrosine, and this conclusion was borne out by two-dimensional paper chromatography of an acid hydrolysate of Fraction I (Fig. 28). Inspection of the chromatogram shows, as would be expected, a preponderance of aspartic and glutamic acids and very little of the basic amino acids among the polar residues. Fraction I also contains some hydroxyproline and a considerable amount of tyrosine, some of which is present in the hydrolysate as the mono-iodo-compound, the latter spot being radioactive. Unfortunately, the yield of Fraction I was too small to allow further fractionation, but experiments are under way to accumulate a sufficient quantity of this fraction for a thorough characterization of the peptides.

In summary, it can be said that the effect of proteases such as trypsin and pepsin on the TC macromolecule appears to be a relatively slight modification, probably in "end regions," which, while leaving intact the helical configuration of the polypeptide chains comprising the macromolecule, drastically modifies its end-to-end polymerization properties. These changes in the "reactivity" of the macromolecule are accompanied by the liberation of a peptide or peptides relatively rich in tyrosine. The results to date are completely compatible with the "end-chain" mechanism for end-to-end polymerization of $\mathrm{TC}$ and suggest that the small tyrosine content of collagen may be importantly involved in the mechanism by which such "end-chains" interact in orderly fashion during polymerization.

\section{References}

BeAR, R S. 1952. The structure of collagen fibrils. Advances in Protein Chem. 7: 69: 160 .

BEAR, R. S., and R. S. Morgan. 1957. The composition of bands and interbands of collagen. In R. E. Tunbridge (ed.). Connective Tissue. Blackwell Scientific Publications, Oxford. Pp. 321-333. 
Bensusan, H. B. 1959. Effect of iodination of procollagen on the formation of fibers. Federation Proc. 18: 190.

Bensusan, H. B., and B. L. Hoyt, 1958. The effect of various parameters on the rate of formation of fibers from collagen solutions. J. Am. Chem. Soc. 80: 719-724.

Boedtker, H., and P. Doty. 1956. The native and denatured states of soluble collagen. J. Am. Chem. Soc. 78: 4267-4280.

Doty, P., and T. Nishihara. 1958. The molecular properties and thermal stability of soluble collagens. In G. Stainsby (ed.). Recent Advances in Gelatin and Glue Research. Pergamon Press, Ltd., London. Pp. 92-99.

Glimcher, M. J., A. J. Hodge, and F. O. Schmitt. 1957. Macromolecular aggregation states in relation to mineralization: The collagen-hydroxyapatite system as studied in vitro. Proc. Nat. Acad. Sci. U. S. 43: 860-867.

Grassmann, W., K. Hannig, H. Endres, and A. Riedel. 1956. Aminosäuresequenzen des Kollagens. I. Zur Bindungsweise der Prolins und Hydroxyprolins. HoppeSeyler's Z. physiol. Chem. 306: 123-131.

Gross, J. 1956. The behavior of collagen units as a model in morphogenesis. J. Biophys. Biochem. Cytol. 2 (Suppl. ): 261-274.

Gross, J. 1958. Studies on the formation of collagen. I. Properties and fractionation of neutral salt extracts of normal guinea pig connective tissue. J. Exptl. Med. 107: 247-263.

Gross, J. 1959. On the significance of the soluble collagens. In I. H. Page (ed.). Connective Tissue, Thrombosis and Atherosclerosis. Academic Press, Inc., New York. Pp. 77-95.

Gross, J., J. H. Highberger, and F. O. Schmitt. 1954. Collagen structures considered as states of aggregation of a kinetic unit. The tropocollagen particle. Proc. Nat. Acad. Sci. U.S. 40: 679-688.

HALL, C. E., and P. Doty. 1958. A comparison between the dimensions of some macromolecules determined by electron microscopy and by physical chemical methods. J. Am. Chem. Soc. 80: 1269-1274.

Highberger, J. H. 1956. The chemical structure and macromolecular organization of the skin proteins. In F. O'Flaherty, W. T. Roddy, and R. M. Lollar (eds.). The Chemistry and Technology of Leather. Reinhold Publishing Corp., New York. Pp. 65-193.

Highberger, J. H., J. Gross, and F. O. Schuntt. 1951. The interaction of mucoprotein with soluble collagen: An electron microscope study. Proc. Nat. Acad. Sci. U. S. 37: 286-291.

Hodge, A. J. 1959a. Fibrous proteins of muscle. Revs. Modern Phys. 31: 409-425.

Hodge, A. J. 1959b. Principles of ordering in fibrous systems. In W. Bargmann et al. (eds.). Proc. Fourth Intern. Congress on Electron Microscopy, Berlin, 1960. Springer Verlag, Berlin.

Hodge, A. J., J. H. Highberger, G. G. J. Deffner, and F. O. Schmitt. 1960. The effects of proteases on the tropocollagen macromolecule and on its aggregation properties, Proc. Nat. Acad. Sci. U. S. 46: 197-206.

Hodge, A. J., and F. O. Schmitt. 1958. Interaction properties of sonically fragmented collagen macromolecules. Proc. Nat. Acad. Sci. U. S. 44: 418-424.

Hodge, A. J., and F. O. Schmitt. 1960. The charge profile of the tropocollagen macromolecule and the packing arrangement in native-type collagen fibrils. Proc. Nat. Acad. Sci. U. S. 46: 186-197.

Küun, K. 1958. Über die Ausbildung einer hochunterteilten Querstreifung des Kollagens nach Gerben mit Phosphorwolframsäure und basischen Chromsalzlösungen. Leder 9: 217-222.

Künn, K., IV. Grassmann, and V. Hofmann. 1957. Über die Bindung der Phosphorwolframsäure im Kollagen. Naturwiss, 44: 538-539.

Nisminara, T., and P. Doty. 1958. The sonic fragmentation of collagen macromolecules. Proc. Nat. Acad. Sci. U. S. 44: 411-417.

Orekovitc1, V. N., and V. O. Shpikiter. 1958. Procollagens. Science 127: 13711376. 
Rich, A., and F. H. C. Crick. 1958. The structure of collagen. In G. Stainsby (ed.). Recent Advances in Gelatin and Glue Research. Pergamon Press, Ltd., London. Pp. 20-24.

Schмiтt, F. O. 1959. Interaction properties of elongate protein macromolecules with particular reference to collagen (tropocollagen). Revs. Modern Phys. 31: 349-358.

Schnett, F. O., J. Gross, and J. H. Highberger. 1953. A new particle type in certain connective tissue extracts. Proc. Nat. Acad. Sci. U. S. 39: 459-470.

Schmitt, F. O., J. Gross, and J. H. Highberger. 1955. States of aggregation of collagen. Symposia Soc. Exptl. Biol. 9: 148-162.

Schmitt, F. O., and A. J. Hodge. 1960. Das Tropokollagen-Makromolekül und die Eigenschaften seiner geordneten Aggregationsformen. Leder. 11: 74-91.

Seifter, S., P. M. Gallop, and E. Meilman. 1958. The degradation of ichthyocol by bacterial collagenase. In G. Stainsby (ed.). Recent Advances in Gelatin and Glue Research. Pergamon Press, Ltd., London. Pp. 164-172.

\section{DISCUSSION}

\section{W. D. McElroy, A. J. Hodge, W. H. Johnson, C. L. Prosser, F. G. Sherman}

Dr. McElroy (Johns Hopkins University): Could you describe the ATP effect in greater detail? Does inorganic pyrophosphate or triphosphate work? What does the addition of $\mathrm{Mg}^{++}$do to this process?

Dr. Hodge: ATP is not unique in its ability to produce SLS-type aggregates from acid solutions of collagen; ITP and nucleic acids will also yield segment-type structures under appropriate conditions. In fact, the SLS form was first discovered by Schmitt et al. (1953) when they dialyzed a phosphate extract of skin collagen $(\mathrm{pH}=8, \mu=0.4)$ against citrate buffer ( $\mathrm{pH} 4$, $\mu=0.2$ ). Such extracts show strong absorption at about $2600 \AA$. The sodium salts of ATP and ITP do not work, nor does inorganic pyrophosphate or triphosphate. These results suggest that the binding of ATP to the TC macromolecules modifies the charge distribution in a way such that (a) the isoelectric point of the TC is lowered and (b) the parallel, in-register SLS-type of packing is favored over the normal staggered configuration of native-type collagen. The presence of appreciable concentrations of salts prevents SLS formation, and SLS-type structures are redissolved if salt is added, suggesting that the linkages involved are electrostatic and relatively weak. I know of no special effect of $\mathrm{Mg}^{++}$on the SLS system, other than its contribution to the ionic strength of the medium.

Dr. Johnson (University of Illinois): Do you think that spatial separation of charged sequences along the molecule would account for periodicities seen in electron micrographs of precipitated preparations of other proteins, such as paramyosin, tropomyosin, and light meromyosin? We have evidence for the presence of polyglutamic acid along the paramyosin molecule. Could this account for periodicity in paramyosin?

Dr. Hodge: Yes. The band patterns observed in the various ordered aggregation states of proteins such as paramyosin, tropomyosin, and light meromyosin are almost certainly due to a precise distribution of "clusters" of polar residues separated axially by regions of non-polar residues. As is the case for collagen, it is the precise spatial separation of these polar regions and their 
charge characteristics which are responsible for (a) the fact that several different stable aggregation states can be formed under appropriate conditions (see Hodge, 1959a, 1959b, for summaries) and (b) the sharpness of the various band patterns. The presence of short sequences of polyglutamic acid in the paramyosin macromolecule would be in accord with this concept, especially since this is an $\alpha$-type protein, in which such polar "clusters" must be formed by the coiling of a single polypeptide chain; in the tropocollagen macromolecule, on the other hand, such polar "clusters" could result from the mutual coiling of three separate polypeptide chains, and the probability of finding polypolar sequences would be less.

Dr. Prosser (University of Illinois): I am still not clear regarding the ATP effect on collagen polymerization. If it is a matter of charge distribution, the polyanions should be effective. Is energy liberated? Is the ATP split?

Dr. Hodge: The effect of ATP on the ordered aggregation of TC macromolecules is unquestionably a matter of charge distribution, and polyanions, such as nucleic acid, are effective in inducing the formation of SLS-type structures. However, the system is rather delicately balanced with respect to $\mathrm{pH}$ and ionic strength, so that the $\mathrm{pK}$ of the inducing agent is fairly critical. As far as I know, SLS formation does not involve liberation of energy from splitting of ATP.

Dr. Sherman (Brown University): Is $\mathrm{Mg}^{++}$required for the ATP effect?

Dr. Hodge: $\mathrm{Mg}^{++}$is not required for the formation of SLS-type aggregates, and indeed, would probably inhibit the precipitation because of its relatively high contribution to the ionic strength of the medium. 


\title{
The Role of the Macromolecular Aggregation State and Reactivity of Collagen in Calcification ${ }^{1}$
}

\author{
Melvin J. Glimcher ${ }^{2}$
}

The mineralization of certain specialized tissues of living organisms is a widespread biological phenomenon (Table 1) which serves a number of highly important mechanical and chemical functions.

It has long been recognized, and recently emphasized by the newer techniques of x-ray diffraction and electron microscopy, that an intimate relationship exists between the mineral crystallites and the organic matrix of these tissues (Schmidt, 1935; Robinson, 1952; Robinson and Watson, 1952, 1955; Finean and Engstrom, 1953; Carlstrom and Finean, 1954; Carlstrom et al., 1955; Speckman and Norris, 1957; Gregoire, 1957; Tsujii et al., 1958; Watabe et al., 1958). Such observations have naturally led investigators interested in the mechanism of tissue mineralization to study the role of the organic matrix in initiating the formation of the inorganic crystals (Freudenberg and Gyorgy, 1921; Gutman and Yu, 1950; Rubin and Howard, 1951; Gersh, 1952; Neuman and Neuman, 1953; Engel et al., 1953; Sobel and Burger, 1954; Sobel, 1955; Strates et al., 1957; Glimcher

${ }^{1}$ These studies were aided by research grants RG 6391 from the Division of General Medical Sciences, RG (Mult) 6391 from the National Institute of Dental Research, H-4777 from the National Heart Institute, and RG 6391-S1 from the National Institute of Arthritis and Metabolic Diseases of the National Institutes of Health, Public Health Service, U. S. Department of Health, Education, and Welfare; and by research grants from the John A. Hartford Foundation Inc., the Easter Seal Research Foundation of the National Society for Crippled Children and Adults, Inc., the Liberty Mutual Insurance Company, and the Orthopedic Research and Education Foundation. Some of the work described was carried out at the Massachusetts Institute of Technology, Cambridge, Massachusetts, and was supported by research grants E-1469, from the National Institute of Allergy and Infectious Diseases, and A-2317, from the ${ }^{\prime}$ National Institute of Arthritis and Metabolic Diseases, National Institutes of Health, Public Health Service, United States Department of Health, Education and Welfare, to the Massachusetts Institute of Technology (Prof. Francis O. Schmitt).

${ }^{2}$ Research Fellow of the Medical Foundation of Boston, Boston, Massachusetts. 
et al., 1957; Strates and Neurnan, 1958). In our laboratory, we have been primarily concerned with one particular mineralizing system: the deposition of the calcium phosphate crystals of apatite in an organic matrix of collagen such as occurs in bone, cartilage, and tooth.

\section{TABLE ।}

Examples of Biologically Mineralized Tissues

(Glimcher, 1959)

\begin{tabular}{|c|c|c|c|c|}
\hline Species & $\begin{array}{c}\text { Tissue } \\
\text { Mineralized }\end{array}$ & $\begin{array}{c}\text { Crystal } \\
\text { Chemistry }\end{array}$ & $\begin{array}{l}\text { Mineral } \\
\text { Form }\end{array}$ & $\begin{array}{c}\text { Major } \\
\text { Organic- } \\
\text { Matrix } \\
\text { Components }\end{array}$ \\
\hline Plants.... & . . Cell wall & $\mathrm{CaCO}_{3}$ & Calcite & $\begin{array}{l}\text { Cellulose, } \\
\text { pectins, } \\
\text { lignin }\end{array}$ \\
\hline Radiolarians & ..Exoskeleton & $\mathrm{SrSO}_{4}$ & Celestite & $(?)^{0}$ \\
\hline Diatoms ... & ..Exoskeleton & Silica & (?) & Pectins \\
\hline Mollusks ... & ..Exoskeleton & $\mathrm{CaCO}_{3}$ & $\begin{array}{l}\text { Calcite, } \\
\text { aragonite }\end{array}$ & $\begin{array}{l}\text { Conchiolin } \\
\text { (protein) }\end{array}$ \\
\hline Arthropods & ..Exoskeleton & $\mathrm{CaCO}_{3}$ & Calcite & $\begin{array}{l}\text { Conchiolin } \\
\text { (protein) }\end{array}$ \\
\hline \multirow[t]{7}{*}{ Vertebrates } & .. Endoskeleton & & & \\
\hline & Bone & $\mathrm{Ca}_{10}\left(\mathrm{PO}_{4}\right)_{6}(\mathrm{OH})_{2}$ & Hydroxyapatite & Collagen \\
\hline & Cartilage & $\mathrm{Ca}_{10}\left(\mathrm{PO}_{4}\right)_{6}(\mathrm{OH})_{2}$ & Hydroxyapatite & $\begin{array}{l}\text { Collagen, acid } \\
\text { mucopoly- } \\
\text { saccharides }\end{array}$ \\
\hline & Tooth & & & \\
\hline & Dentin & $\mathrm{Ca}_{10}\left(\mathrm{PO}_{4}\right)_{6}(\mathrm{OH})_{2}$ & Hydroxyapatite & Collagen \\
\hline & Cementum & $\mathrm{Ca}_{10}\left(\mathrm{PO}_{4}\right)_{6}(\mathrm{OH})_{2}$ & Hydroxyapatite & Collagen \\
\hline & Enamel & $\mathrm{Ca}_{10}\left(\mathrm{PO}_{4}\right)_{6}(\mathrm{OH})_{2}$ & Hydroxyapatite & Eukeratin \\
\hline
\end{tabular}

As a result of a series of experiments carried out at the Massachusetts Institute of Technology in collaboration with Professor Francis O. Schmitt and Dr. Alan J. Hodge, a general concept of the basic physicochemical mechanism initiating calcification has been postulated, based on the observations of the specific interrelations between native and reconstituted collagens and metastable calcium phosphate solutions, and on a companion x-ray diffraction and electron optical study of both developing and adult bone (Glimcher et al., 1957; Glimcher, 1958; Glimcher, 1959; Glimcher et al., 1960a, 1960h). While only the collagen-hydroxyapatite system has thus far been given careful experimental study, the basic hypothesis may apply to the problem of biological mineralization in general. Comprehensive reviews of these concepts and experiments have recently 
been presented (Glimcher et al., 1957; Glimcher 1958; Glimcher, 1959; Glimcher et al., 1960a, 1960b) and therefore need only be summarized here.

The basis of the hypothesis rests on an understanding of the physicochemical phenomena involved in phase transitions, of which crystallization is just one specific case (Turnbull, 1956; Glimcher, 1959). A common example of a phase transition is the formation of ice crystals from liquid water or from supersaturated water vapor. The use of AgI crystals in "seeding" supersaturated water vapor to initiate ice crystal formation is well known. The formation of a new phase (i.e., ice from supersaturated vapor) by the use or introduction of such a seeding material is called heterogeneous nucleation. Two important points should be noted: (1) The water vapor or liquid water must be supersaturated, that is, in metastable equilibrium; systems in metastable equilibrium may remain stable indefinitely yet still retain the ability to form a new phase under the proper conditions, "seeding," for example. (2) The "seed" crystal must have an atomic lattice structure similar to that of the crystal being nucleated (Turnbull, 1950a, 1950b, 1952; Turnbull and Vonnegut, 1952); in the case of AgI and ice, for example, there is a remarkably close fit between their lattice parameters.

Examination of the biological conditions as they relate to calcification of bone, cartilage, and tooth reveals some interesting correlations. The extracellular fluids of many vertebrates have been shown to be in metastable equilibrium with respect to the formation of apatite crystals (Neuman, 1950; Strates et al., 1957; Strates and Neuman, 1958; Glimcher, 1959). Furthermore, the major organic component of bone, of cartilage, and of the dentin and cementum of tooth is the fibrous protein, collagen. This complex macromolecule, which is thought to be composed of three polypeptide chains in a coiled-coil configuration (Rich and Crick, 1955, 1958), is so structurally regular that it gives rise to a characteristic $\mathrm{x}$-ray diffraction pattern and therefore for our purpose may be considered "crystalline" (Fig. 1). In addition, the native collagen fibril, which is composed of such macromolecules aggregated both linearly and longitudinally in a highly specific and characteristic fashion, is therefore also a well-ordered structure and may also be considered "crystalline" (Fig. 2). When to such data is added the observation of the intimate association between the apatite crystals and collagen (the inorganic apatite crystals in bone are located primarily within 


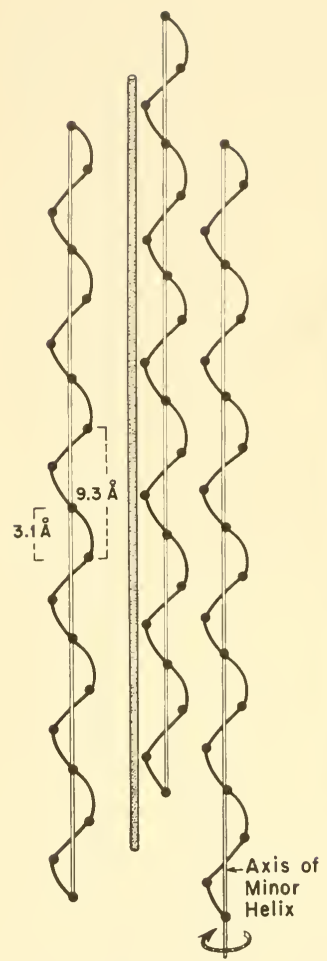

A

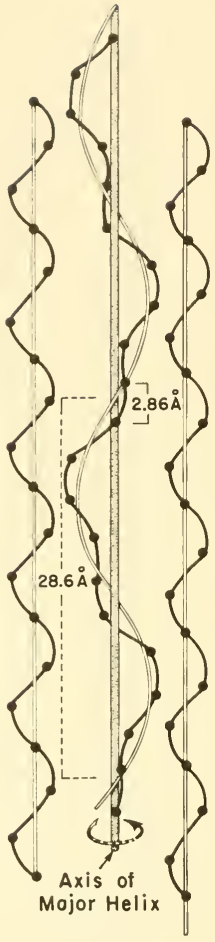

B
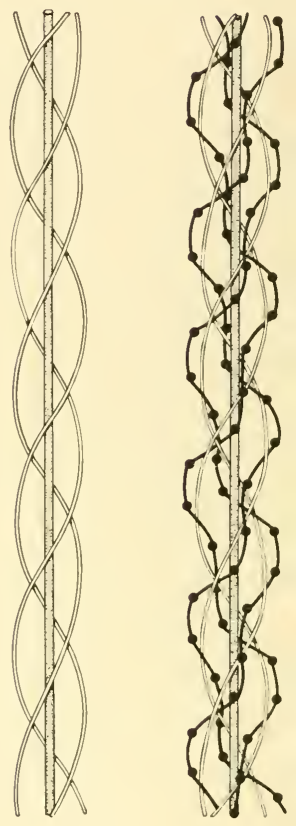

C
D

Fig. 1. (A), (B), (C), (D) Diagrammatic derivation of the coiled-coil structure of the collagen macromolecule. Each of the three polypeptide chains is wound about its own axis as a left-handed helix in a threefold screw fashion. The axes are located at the vertices of an equilateral triangle and are parallel. The

the collagen fibrils; see Glimcher, 1959, Glimcher et al., 1960a, and Figs. 3, 4, 5), the possibility that either the macromolecular structure or the macromolecular aggregation state of collagen is responsible for the induction of calcification by acting as a catalytic heterogeneity ( similar to AgI in the case of supersaturated water vapor) 
screw repeat in the case of polyglycine, for example, is $9.3 \AA$, and the rise per residue (each of which is represented here by a black dot) is $3.1 \AA \AA$. (E) X-ray diffraction pattern of oriented collagen fibers.

is apparent. The concept that one or more components of the organic matrix of bone, cartilage, and tooth catalyzed the formation of apatite crystals was first proposed by Neuman and Neuman (1953).

From solutions of collagen extracted from a wide variety of con- 


\section{\|ninown.nil|}

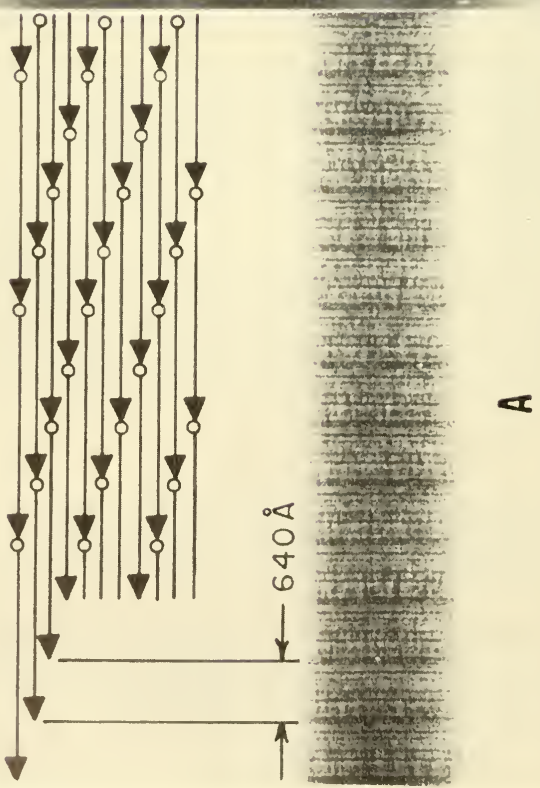




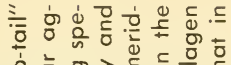
t.

过

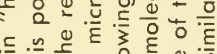

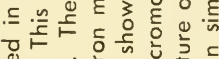

为

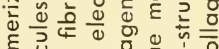

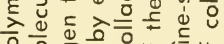

잉

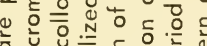

ठั ปั

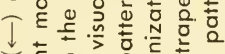

衣은

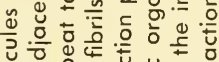

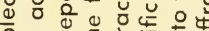

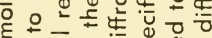

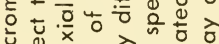

응 인 인

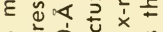

둥ㅇㅇ으는

o눈

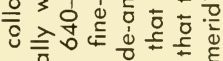

든

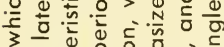

$3-$ 은 응 응

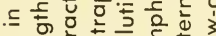

흐을

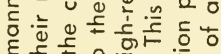

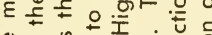

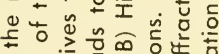

ডั

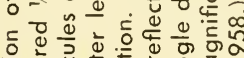
ब O $О$ O

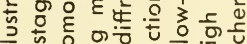

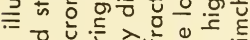

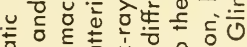

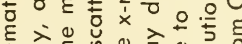

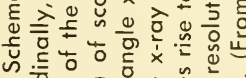

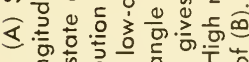

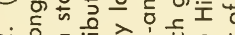

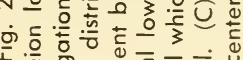
证告

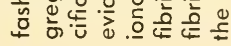




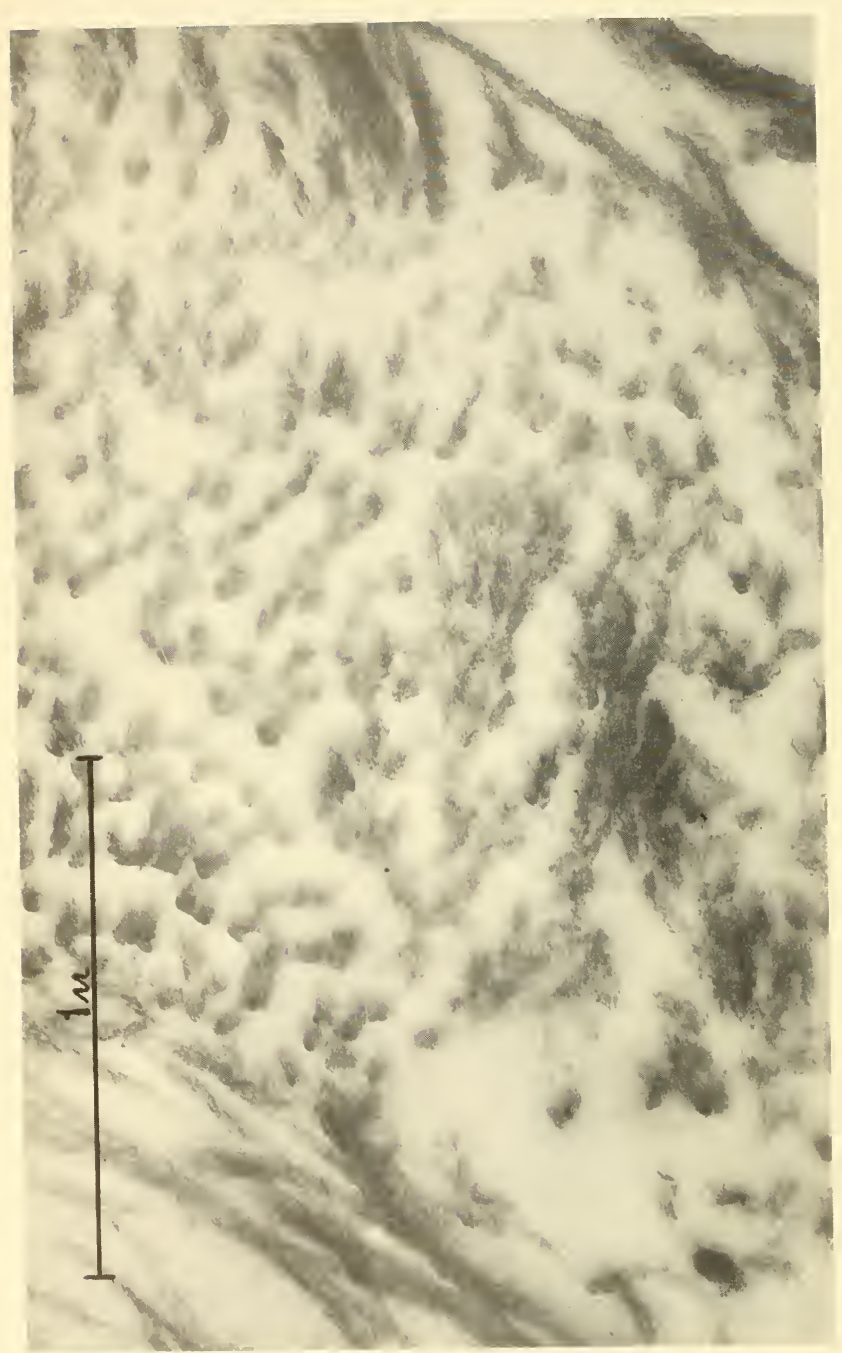

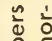

당

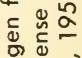

宅过

$\stackrel{5}{ \pm}$

$\leftarrow \div$

등ํำ

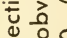

능

D. $=\infty$

든 $x$

屯

o

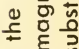

.

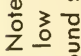

i. $\cong$ 은

둥

으을

ज

प

ㄴ.

은 은

空

ڤ 0.

崩产

는

○

ডั워

드은

히ㅇㅝㅗ

인

ह

ᄃ

은 응

는

ш $\frac{\frac{1}{5}}{\frac{0}{5}}$

ल

ㅎํ

호 든 는 ᄃ욤 


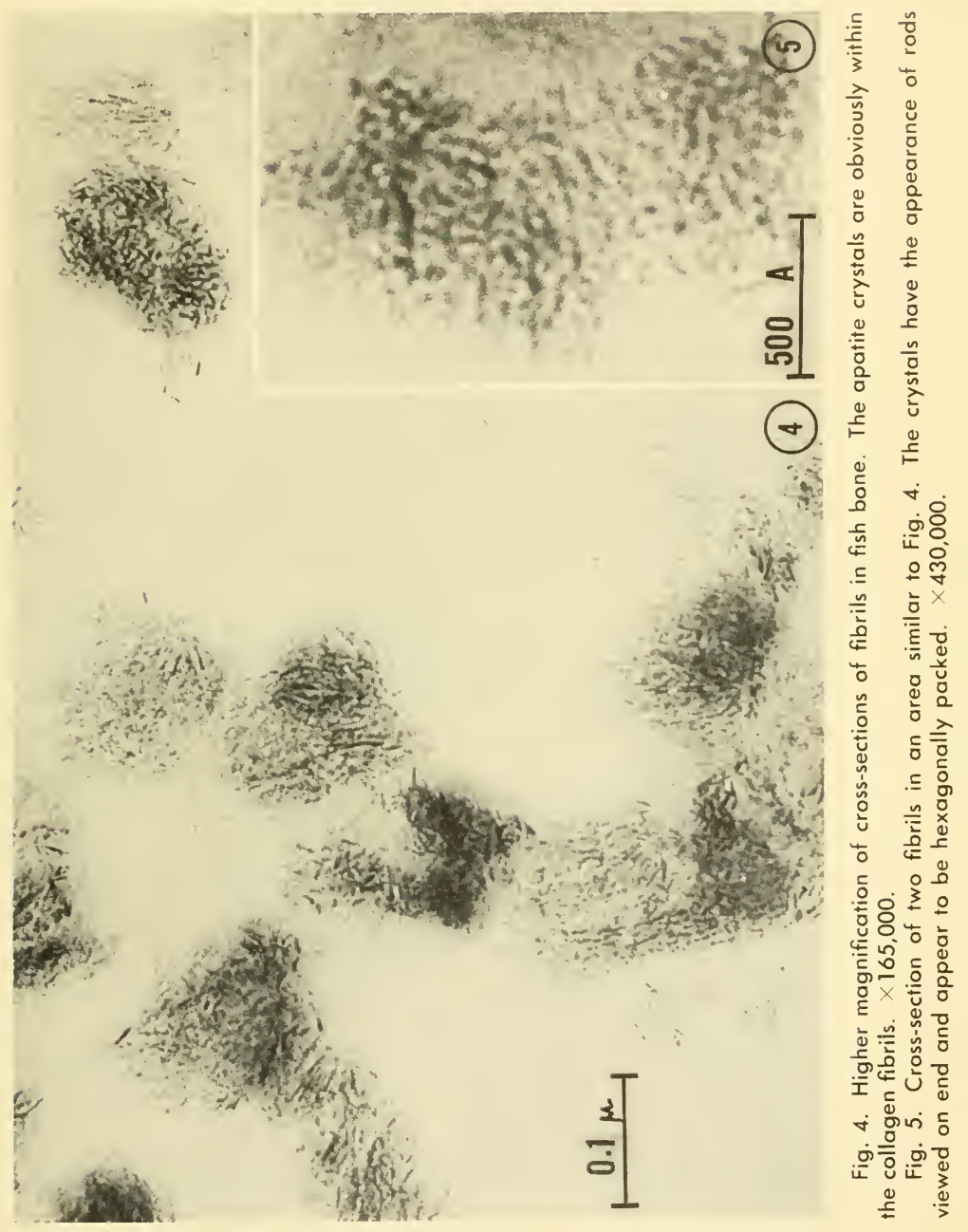


nective tissues it is possible to reconstitute in vitro not only the native-type (640-700- $\AA$ axial repeat) collagen fibrils, but also a number of other fibrillar forms (Schmitt et al., 1942; Highberger et al., 1951; Gross et al., 1954, 1955; Schmitt et al., 1955a, 1955b). These various fibrillar forms represent different aggregation states of the same macromolecules and therefore reflect differences between the stereochemical relations of the amino acid sidechain groups of adjacent macromolecules. Since it is also possible to isolate the macromolecules and linear aggregates of the macromolecules (protofibrils), we were thus provided with a unique system which would not only test the nucleation hypothesis, but which also could distinguish whether this property (if such existed) was a function of the structure of the macromolecule itself or was dependent on steric factors and a characteristic electrical charge distribution provided by the aggregation of the macromolecules into fibrils (Fig. 6).

The experiments were conducted by exposing the various types of fibrils, macromolecules, and protofibrils, as well as previously demineralized bone collagen (shown by low-angle $\mathrm{x}$-ray diffraction to have maintained its 640 - $\AA$ long-range fibrillar order) to metastable calcium phosphate solutions.

Under identical physicochemical conditions, only the native-type (640-700- $\AA$ axial repeat) reconstituted collagen fibrils and the demineralized bone collagen were able to nucleate apatite crystals from metastable calcium phosphate solutions. The failure of the various other fibrils, representing different aggregation states of the same macromolecules, to initiate this phase change demonstrated the rather remarkable steric specificity required in the nucleation phenomenon. Since it was also demonstrated that the individual tropocollagen macromolecules and the protofibrils were unable to initiate crystallization, it was evident that the specificity did not lie in the macromolecule itself, but in the specific manner in which the collagen macromolecules were packed to make native fibrils, i.e., their macromolecular aggregation state. The structural matching in the case of collagen depends in the first instance upon the integrity of its molecular structure, which assures a specific linear array of amino acid residues in the covalent polypeptide chains. Yet even when this condition is met it is not sufficient to provide a nucleation site. Nucleation occurs only when the tropocollagen particles are aligned both laterally and longitudinally in the specific array char- 
acteristic of the native-type collagen fibril, leading to a precise juxtaposition of amino acid side-chain groups from adjacent macromolecules.

Additional confirmation of this steric requisite was obtained by subjecting aliquots of reconstituted native-type collagen fibrils and
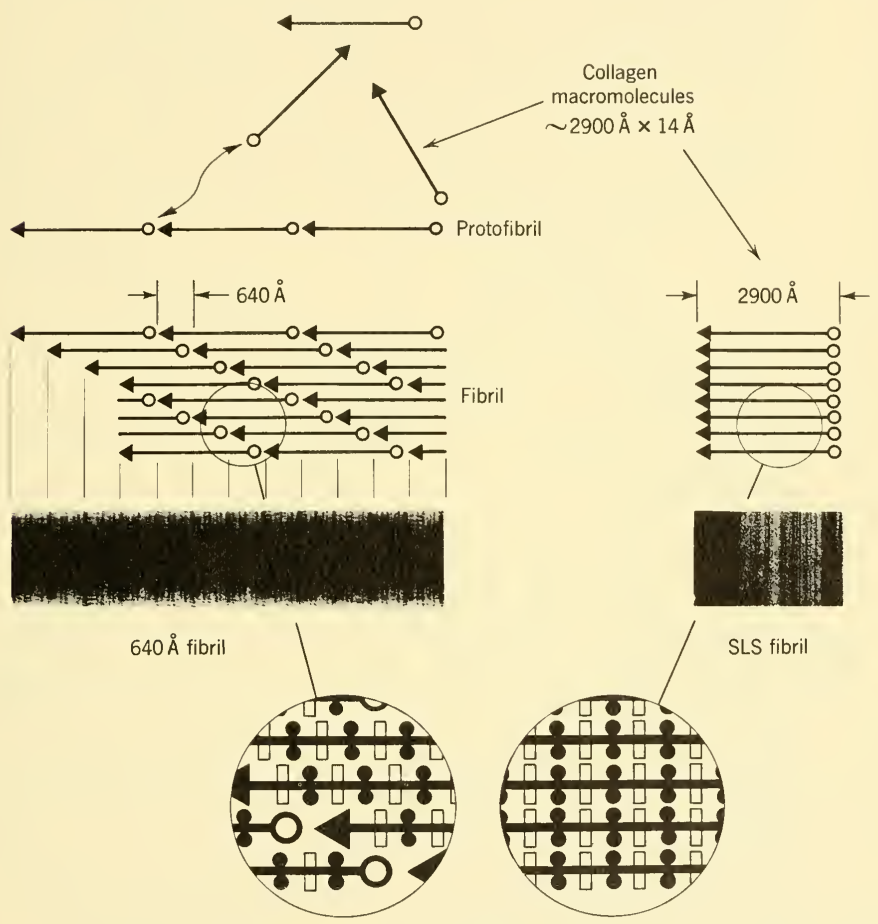

Fig. 6. The steric differences between the reactive side-chain groups of adjacent macromolecules in the native and SLS forms of collagen, diagrammatically portrayed. (From Glimcher, 1959.)

of demineralized bone collagen to a number of physical and chemical agents which destroyed the ordered structure of the fibrils without altering the reactivity of the amino acid side-chain groups. These fibrils lost the ability to induce crystallization (Fig. 7).

From the macromolecular organization of the collagen fibrils 
and from the fact that a precise stereochemical configuration of a number of amino acid side-chains was necessary to form a nucleus, it was reasonable to expect that there should be a limited number of such preferred centers and that they should occur periodically both laterally and longitudinally along the axial repeat structure of

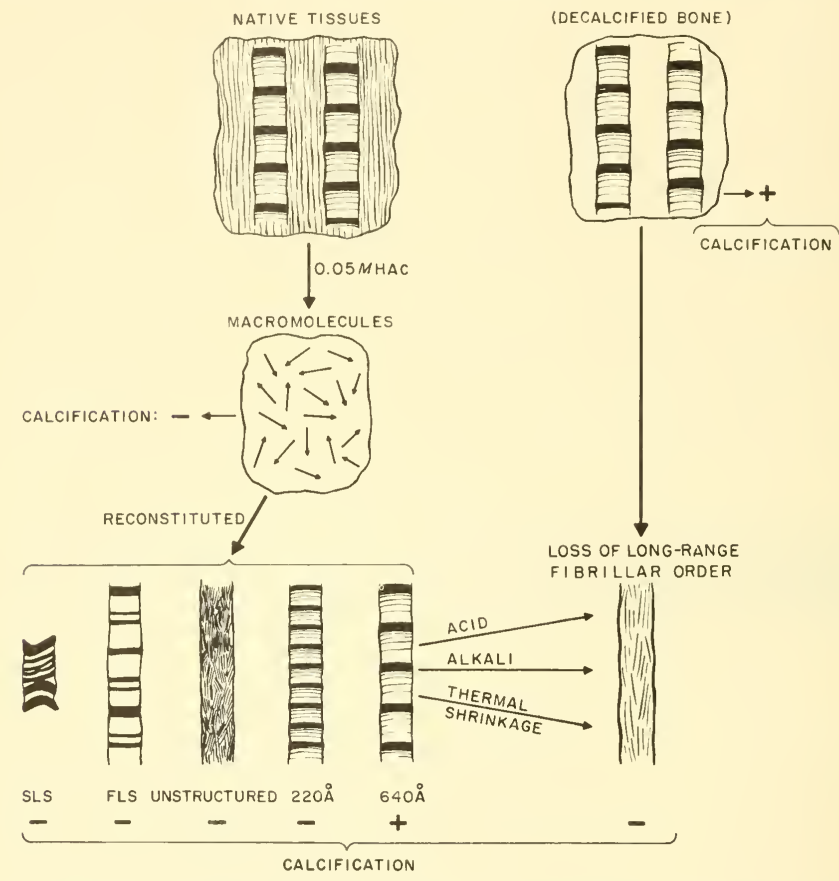

Fig. 7. Some of the experiments which demonstrated the specificity of the macromolecular aggregation state of collagen in in vitro calcification, diagrammatically illustrated. (From Glimcher, 1958.)

the collagen fibrils. Confirmation of the existence of such discrete nucleation centers has come from a time-study of the nucleation process, both by electron microscopy and by low-angle x-ray diffraction. Using the former technique, we have been able to visualize the initial stages of mineralization in vitro. These appear very similar to the initial stages of mineralization in embryonic bone (Figs. 8, 9, 10), and emphasize the regularity of the initial crystal- 


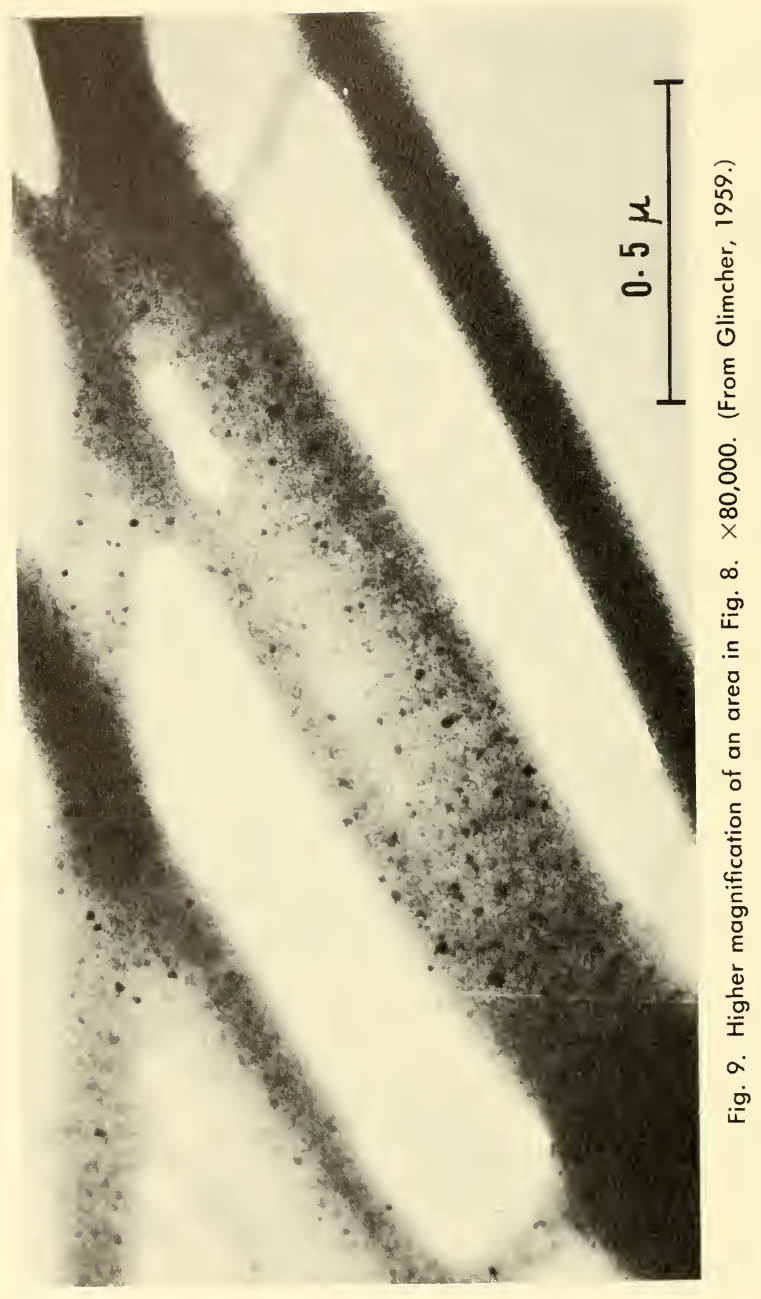




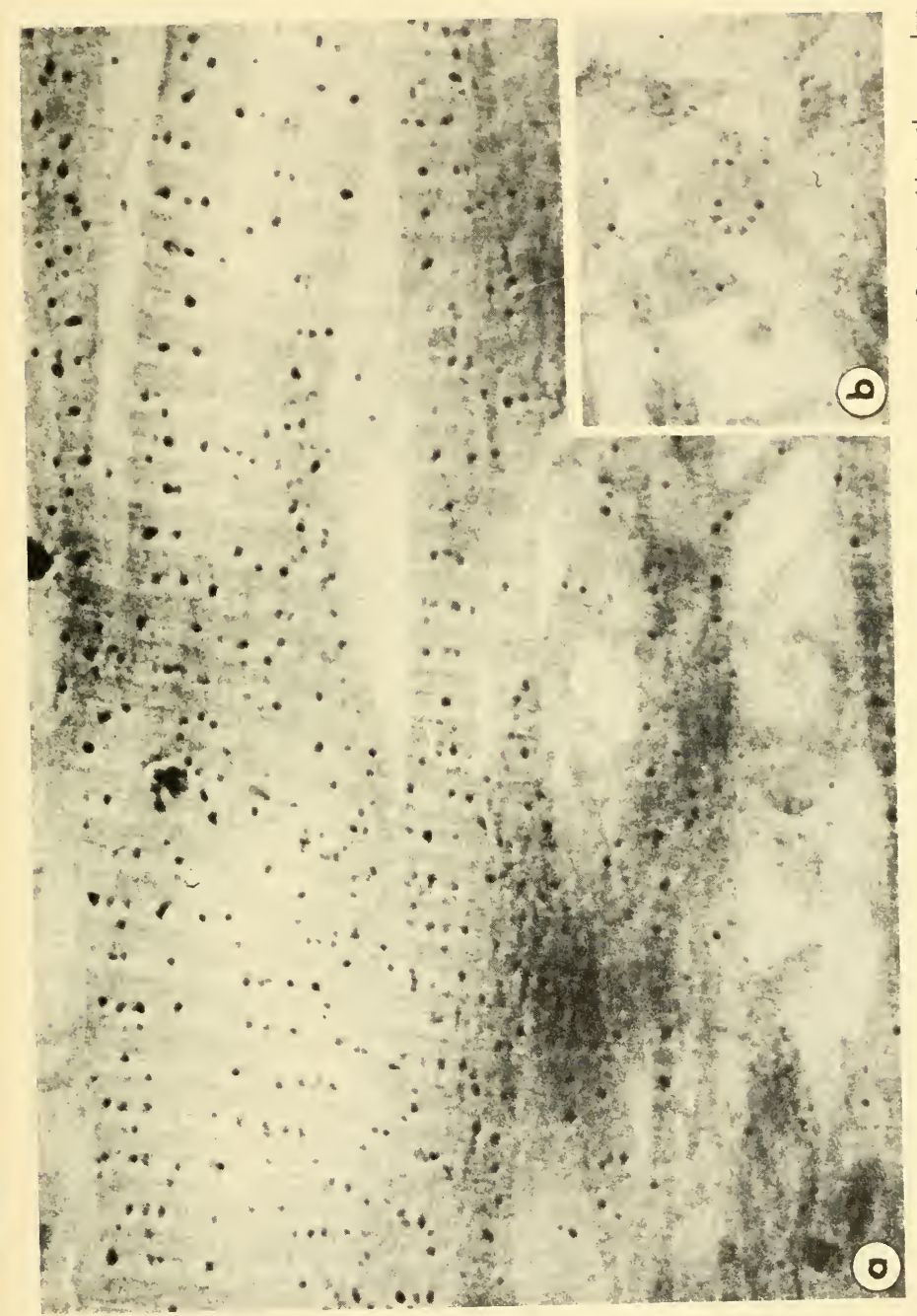

흘 ㅇํำ

인

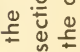

$\frac{1}{0}$ ○े

Z

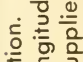

苞

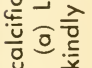

는

\& 은

कृ

호응

ธ్ ठำ

은 $\overline{0}$

엉

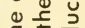

$\div$

ᄃำ응

Ф

응

.

은

을 옳

(1) 응으

등ํํ

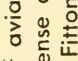
잉

동릉 ํํㅇ

잉느

늘

ㄷํㅇㅇㅛ

은 은 -ㅡㄴ

는

을 능

.

कं :

is

ชั 
lization sites in the collagen fibrils. Preliminary studies by lowangle $x$-ray diffraction, in which the changes in the intensities of the diffraction spacings were measured, have also indicated that there are specific sites in the collagen fibrils which act as nucleation centers. $^{3}$

Obviously, the next step in understanding the nucleation process would be to discover which amino acid side-chain groups interact with which mineral ions, the nature of the intermolecular forces between collagen and the mineral ions, and the kinetics and atomic sequence of the nucleation process itself. Unfortunately, even in very simple systems, little is known about such phenomena, and more attention has been paid to nucleation rates than to how the nuclei themselves are formed. However, since it is possible that the initiation and sequence of nucleation may be enzymatically controlled and regulated in biological mineralization, such information is of crucial importance.

As to the specific organic groups and mineral ions involved in calcification, most investigators in the past have felt that the initial step involved the combining of an anionic organic group with calcium ion (Rubin and Howard, 1951; DiStefano et al., 1953; Sobel and Burger, 1954; Sobel, 1955). However, there are some considerations which cast doubt on the hypothesis of the primary role of calcium-binding in crystal induction.

The case for the importance of the phosphate ion has been summarized excellently by McLean (1958). To such points as the importance of organic phosphate compounds for energy transfer (high-energy phosphate bonds) and the possible enzymatic control of such reactions, might be added some structural considerations as well. Namely, the apatites are essentially phosphate salts with structural characteristics primarily attributable to the phosphate groups and not to calcium atoms; the latter can be replaced by a number of other cations, such as strontium and lead, without changing the major features of the crystal structure or symmetry. In addition, if cellular-controlled, enzymatic transfers of mineral ions to specific groups in the collagen are involved in the nucleation process, phosphate is again the most likely candidate. No calcium-splitting enzymes or calcium transferases have as yet been described, al-

${ }^{3}$ Many of the low-angle $x$-ray diffraction studies were carried out in cooperation with Dr. A. Posner, American Dental Association, Research Division, National Bureau of Standards, and with Dr. L. Bonar, Department of Biophysics, Massachusetts Institute of Technology. 
though there are a host of such enzymes for phosphate. Furthermore, if $\mathrm{Ca}^{++}$were transferred enzymatically to the collagen, any covalent chelating or co-ordinating structure formed would be very unreactive, i.e., would not be very accessible to reaction with the other ions making up the crystal lattice; such bonds would tend to demineralize ( similarly to other chelates like ethylenediaminetetracetic acid, EDTA), rather than to initiate nuclei formation. On the other hand, covalent phosphate compounds such as -N-P or -O-P alkyl amides or esters would still be very reactive, with $\mathrm{Ca}^{++}$for example. Since the phosphate groups are not only the structural "backbone" of the apatite lattice, but are also more likely to be involved from the biological standpoint, their role in the formation of the initial fragments of the crystal lattice or nucleus would appear to be equally as important as that of the calcium ions, and probably more important. Indeed, the proposal that phosphorylation of free amino groups constitutes the initial step in calcification has been made in the past (Polonovski and Cartier, 1951; Cartier, 1951, 1952; Cartier and Picard, 1955; Zambotti, 1957), and it has been suggested that pyrophosphate radicals are the initially bound phosphate moiety (Cartier, 1959).

Two general methods of attack are possible in an effort to ascertain the reactive amino acids involved in the nucleation phenomena. In the first, which we may term the "availability" method, one may determine the difference (if any) between the number of side-chain groups of a particular amino acid available in the fully mineralized matrix and the number available in demineralized matrix, the assumption being that groups which are not reactive in the mineralized tissue are not available because of interaction with the mineral ions. In the second method, specific amino acid side chains of demineralized collagens are blocked or altered, and the effect of such alterations evaluated by comparing the amount of mineral deposited during in vitro calcification with untreated controls.

Solomons and Irving (1958) have studied the availability of the epsilon amino groups $\left(\epsilon-\mathrm{NH}_{2}\right)$ of lysine and hydroxylysine in bone and dentin by reacting such tissues with 1 :fluoro: $2: 4$ dinitrobenzene (FDNB). They noted that, as bone and dentin were demineralized in stages by a mild chelating agent (EDTA), there was a direct relation between the availability of such groups to FDNB and the amount of total mineral present in the sample (calcium and phosphate) (Fig. 11). 
However, when one carefully considers the interpretations of such experiments, it appears likely that, rather than demonstrating an inorganic-organic linkage, the findings are more consistent with the interpretation that the phenomenon represents a diffusion-limiting process. Some of the detailed arguments and experiments for such an interpretation have already been presented (Glimcher, 1958).

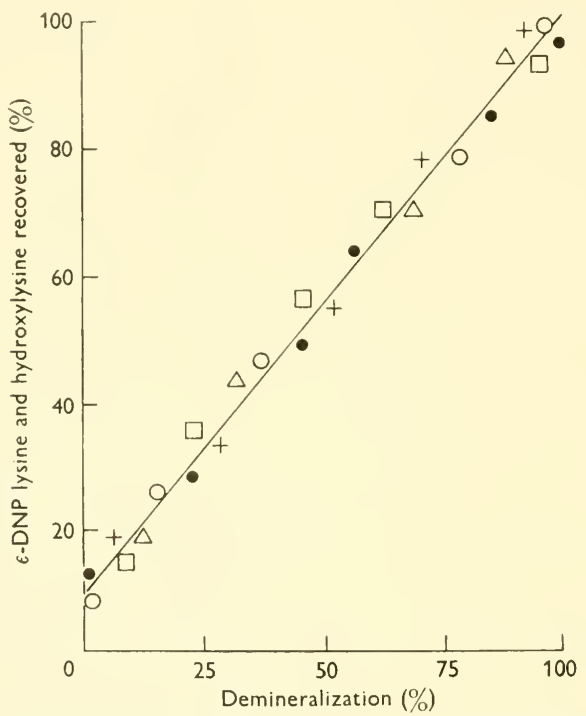

Fig. 11. Availability of $\epsilon$-DNP-lysyl and hydroxylysyl amino groups of hardtissue collagens to FDNB during demineralization. $O$, denote successive demineralization and dinitrophenylation of ox dentin and human dentin, respectively; $+, \triangle, \square$ denote simultaneous demineralization and dinitrophenylation of ox dentin, ox bone, and human dentin, respectively. (From Solomons and Irving, 1958.)

In brief, these experiments showed (1) that the number of $\epsilon-\mathrm{NH}_{2}$ groups in a variety of different bones varied from approximately 35 micromoles per gram of collagen, in oxbone, to approximately 200 250 micromoles per gram of collagen, in fish bone, and was directly related to the packing and density of the tissues and not to the amount of mineral per gram of organic weight, which was essentially the same in all cases (Tables 2A, 2B); (2) that both the number of 
TABLE 2A

Availability of $\epsilon-\mathrm{NH}_{2}$ Groups of Bone Collagen to FDNB

as a Function of Their Mineral Content (Hand-cut, or

"coarse," particles demineralized with EDTA)

\begin{tabular}{ccc}
\hline $\begin{array}{c}\text { Per Cent } \\
\text { Organic Weight }\end{array}$ & $\begin{array}{c}\text { Per Cent } \\
\text { Demineralization }\end{array}$ & $\begin{array}{c}\mu \text { mole } \epsilon \text {-NH } H_{2} \text { Groups } \\
\text { Available/g Collagen }\end{array}$ \\
\hline & FisH Bone & \\
34 & 0 & 193 \\
39 & 8 & 243 \\
52 & 27 & 288 \\
74 & 60 & 305 \\
98 & 97 & 311 \\
& CALF BoNE & \\
37 & 0 & 137 \\
39 & 3 & 179 \\
42 & 8 & 205 \\
53 & 25 & 242 \\
72 & 56 & 261 \\
92 & 87 & 291 \\
96 & 94 & 298 \\
98 & 97 & 304 \\
\hline
\end{tabular}

TABLE $2 B$

Availability of $\epsilon-\mathrm{NH}_{2}$ Groups to FDNB in Native Undecalcified Bones of Various Densities, in Order of Decreasing Density (Hand-cut, or "coarse," particles reacted for 48 hours)

\begin{tabular}{|c|c|c|}
\hline Bone Source & $\begin{array}{l}\text { Per Cent } \\
\text { Mineral }\end{array}$ & $\begin{array}{l}\mu \text { mole } \epsilon-\mathrm{NH}_{2} \text { Groups } \\
\text { Available } \mathrm{g} \text { Collagen }\end{array}$ \\
\hline $\mathrm{Ox}$ & 66.23 & 35 \\
\hline Hen. & 68.20 & 82 \\
\hline Chick & 65.53 & 114 \\
\hline Calf. & 63.10 & 161 \\
\hline Fish $\ldots \ldots \ldots \ldots$ & 64.57 & 216 \\
\hline Embryonic chicken & 56.0 & 263 \\
\hline
\end{tabular}

carboxyl groups (glutamic and aspartic acids) and the number of guanidino groups (arginine) which were available also increased with demineralization (Tables $3 \mathrm{~A}, 4$ ); (3) that the number of such groups available in native bone of various kinds also varied in a fashion similar to the $\epsilon-\mathrm{NH}_{2}$ groups, and was also apparently dependent on the density and packing of the specimen; and (4) that the number of reactive groups available in native bone varied with the particle size: the finer the particle size of the bone samples, the more groups that were available for reaction (Tables $2 \mathrm{C}, 2 \mathrm{D}, 3 \mathrm{~B}$ ). 
TABLE 2C

Availability of $\epsilon-\mathrm{NH}_{2}$ Groups to FDNB in Native Bone of Various Species as a Function of Particle Size

(Reacted for 48 hours)

\begin{tabular}{|c|c|c|}
\hline \multirow[b]{2}{*}{ Bone Source } & \multicolumn{2}{|c|}{$\mu$ mole $\epsilon-\mathrm{NH}_{2}$ Groups Available/g Collagen } \\
\hline & Coarse & Finely Powdered \\
\hline$\ldots \ldots \ldots \ldots \ldots \ldots$ & 35 & 88 \\
\hline$\ldots \ldots \ldots \ldots \ldots \ldots$ & 82 & 116 \\
\hline$\ldots \ldots \ldots \ldots \ldots \ldots \ldots$ & 166 & 204 \\
\hline Fish $\quad \ldots \ldots \ldots \ldots \ldots \ldots \ldots \ldots$ & 216 & 267 \\
\hline Embryonic chicken $\ldots \ldots \ldots \ldots$ & 263 & 323 \\
\hline
\end{tabular}

TABLE 2D

Availability of $\epsilon-\mathrm{NH}_{2}$ Groups to FDNB in Native Bone as a Function of Time of Exposure and Particle Size

\begin{tabular}{|c|c|c|c|c|c|}
\hline & \multirow[b]{3}{*}{ Bone Source } & \multicolumn{4}{|c|}{$\mu$ mole $\epsilon-\mathrm{NH}_{2}$ Groups Available/g Collagen } \\
\hline & & \multicolumn{2}{|c|}{ Coarse } & \multicolumn{2}{|c|}{ Finely Powdered } \\
\hline & & 2 Days & 2 Weeks & 2 Days & 2 Weeks \\
\hline Calf & & 161 & 200 & 203 & 229 \\
\hline \multirow[t]{2}{*}{ Fish } & (a) & 216 & 267 & 247 & 273 \\
\hline & (b) & 193 & 244 & 313 & 312 \\
\hline
\end{tabular}

TABLE 3A

Availability of Guanidino Groups of Arginine in Bone Collagen to Phosphotungstic Acid, as a Function of Mineral Content

(Note that the mineral contents of the native undecalcified bone are approximately the same although they have markedly different densities, ox $>$ fish.)

\begin{tabular}{ccc}
\hline $\begin{array}{c}\text { Per Cent } \\
\text { Organic Weight }\end{array}$ & $\begin{array}{c}\text { Per Cent } \\
\text { Demineralization }\end{array}$ & $\begin{array}{c}\mu \text { mole Arginine } \\
\text { Available g Collagen }\end{array}$ \\
\hline 36.5 & FisH Bone & \\
41.1 & 0 & 144 \\
51.4 & 8 & 162 \\
89.9 & 24 & 217 \\
99.0 & 84 & 300 \\
& 98 & 416 \\
36.8 & Ox BoNE & 42 \\
58.6 & 0 & 124 \\
95.0 & 34 & 309 \\
-100.0 & 92 & 419 \\
\hline
\end{tabular}


TABLE 3B

\section{Availability of Guanidino Groups of Arginine to PTA as a Function of Particle Size in Native Mineralized Bone}

$\mu$ mole of Arginine Available/g Collagen

Bone Source Coarse Powdered

Ox $\ldots \ldots \ldots \ldots \ldots \ldots \ldots \ldots \ldots \ldots \ldots \ldots \ldots$

Fish $\ldots \ldots \ldots \ldots \ldots \ldots \ldots \ldots \ldots \ldots \ldots$

\section{TABLE 4}

\section{Availability of Carboxyl Groups to Methylation in Two Species of Bone as a Function of Mineral Content *}

(Note that the mineral contents of the native undecalcified bones are approximately the same although they have markedly different densities, fish $<$ calf.)

$\begin{array}{ccc}\text { Per Cent } & \text { Per Cent } & \mu \text { mole Carboxyl Groups } \\ \text { Organic Weight } & \text { Demineralization } & \text { Available } / g \text { Collagen }\end{array}$

\section{FISH}

44.0

98.4

46.0

98.5
$11.0+$

97.5

CALF

$14.0 \nmid$

97.5
132

590

《70

595

- The number of groups available (for methylation) depends, among other things, on the volume of solution used, normality of acid, and time of exposure. Therefore, the figures do not represent the total number of groups available, but only that number available under experimental conditions used. Since the two species of bones were treated identically, the values obtained are comparable. Other experiments have shown that in hand-cut, coarse fish bone particles over 70 per cent of the theoretical number of carboxyl groups can be methylated.

$f$ Due to the $\mathrm{HCl}$ in the methylating media and not to EDTA.

Analytical values varied but at the most were well under 70 $\mu \mathrm{mole} / \mathrm{g}$.

In any event, determining the number of $\epsilon-\mathrm{NH}_{2}$ groups which are available for reaction with FDNB will not show whether there is or is not an electrostatic interaction between the $\epsilon-\mathrm{NH}_{2}$ groups and the mineral ion(s). In both instances (free or electrostatically linked $\epsilon-\mathrm{NH}_{2}$ ' groups), the FDNB would react with the $\epsilon-\mathrm{NH}_{2}$ groups, in the latter case by displacing the relatively weak electrostatic bond. On the other hand, failure of the $\epsilon-\mathrm{NH}_{2}$ groups to react with FDNB 
despite the removal of the mineral (and assuming no steric hindrance) could mean that a stable (covalent or coordinate) bond existed between the $\epsilon-\mathrm{NH}_{2}$ groups and one or more of the mineral ions.

On the other hand, group-blocking and -substitution experiments appear to give more conclusive evidence. We are continuing to study the problem by this latter method; after blocking specific amino acids, the ability of the collagen to mineralize in vitro is analyzed, and the quantity of Ca-salts deposited is measured. It is well
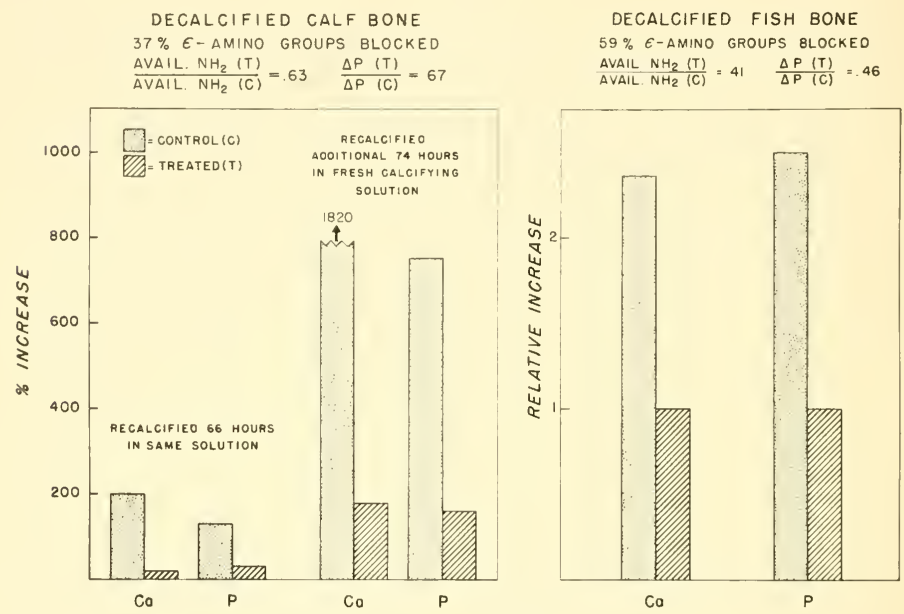

Fig. 12. The effect of blocking the $\epsilon-\mathrm{NH}_{2}$ groups of bone collagen on remineralization in vitro. Collagen treated with carbobenzoxychloride. (From Glimcher, 1958.)

known that it is difficult to pinpoint the active groups in biological molecules which are necessary for a physiological function; in order to be meaningful, experiments designed for this purpose must meet a number of general and specific criteria. These have been sufficiently and thoroughly discussed in the past in relation to the blocking of specific groups in proteins in general (Olcott and FraenkelConrat, 1947; Putnam, 1953) and therefore need not be detailed here.

While it is perfectly clear that our experimental results must therefore be viewed with great caution, they have been encouraging 
enough and sufficiently suggestive to warrant discussion here. Our attention has been primarily concentrated on the $\epsilon-\mathrm{NH}_{2}$ groups of lysine and hydroxylysine partly because this group is quite easily blocked without altering either the molecular structure of collagen or its macromolecular aggregation state and partly because of the likelihood that this group would be involved in phosphate-binding, which we feel is an important step in nucleation. Experiments in which the $\epsilon-\mathrm{NH}_{2}$ groups were specifically blocked in varying amounts by several different reagents showed a definite correlation between the number of $\epsilon-\mathrm{NH}_{2}$ groups available and the total amount of mineral deposited. Fig. 12 and Table 5 give examples of the re-

\section{TABLE 5}

Effect of Blocking the $\epsilon-\mathrm{NH}_{2}$ Groups of Demineralized Fish Bone (Collagen) with 1:Fluoro:2:4 Dinitrobenzene (FDNB) on Its Subsequent Recalcification in vitro

(Recalcified at $25^{\circ} \mathrm{C}$ for approximately 48 hours)

\begin{tabular}{ccc}
\hline Mg Ca g Collagen & Mg P/g Collagen & $\begin{array}{c}\mu \text { mole } \epsilon-\mathrm{NH}_{2} \\
\text { Groups Blocked by } \\
\text { FDNB } / g \text { Collagen }\end{array}$ \\
\hline 45.70 & 28.63 & $0^{\circ}$ \\
33.49 & 19.43 & 53 \\
23.51 & 13.01 & 84 \\
20.52 & 10.89 & 226 \\
18.67 & 9.72 & 286 \\
4.55 & 1.88 & 334
\end{tabular}

- A sham control which was exposed to the same reagents under identical conditions of $\mathrm{pH}$, etc., but without the addition of FDNB.

sults of such experiments. One of the most convincing pieces of evidence thus far was the demonstration that the $\epsilon-\mathrm{NH}_{2}$ groups could be reversibly blocked, and that there was a concomitant change in the amount of mineral deposited (Table 6).

While such experiments indicate that the group substitution has been carried out without destroying the molecular structure of the collagen, they do not rule out the possibility that the observed results are, in fact, due to the shielding (either electrostatic or steric) of groups adjacent to the $\epsilon-\mathrm{NH}_{2}$ groups, rather than to the $\epsilon-\mathrm{NH}_{2}$. groups themselves.

Since availability studies of fully demineralized bone and dentin indicate at least 95 per cent of the $\epsilon-\mathrm{NH}_{2}$ groups are not cova- 
TABLE 6

Reversible Blocking of the $\epsilon-\mathrm{NH}_{2}$ Groups of Reconstituted Collagen

(Ichthyocol) by Carbobenzoxychloride and the Concomitant

Change in Its Recalcification in vitro

\begin{tabular}{|c|c|c|c|}
\hline Specimen & $\begin{array}{l}\mu \text { mole } \epsilon-\mathrm{NH}_{2} \text { Groups } \\
\text { Available/g Collagen }\end{array}$ & $\begin{array}{l}\mathrm{Mg} \mathrm{Ca} \cdot \mathrm{g} \\
\text { Collagen }\end{array}$ & $\begin{array}{l}\mathrm{Mg} \mathrm{P} / \mathrm{g} \\
\text { Collagen }\end{array}$ \\
\hline \multicolumn{4}{|c|}{ Calcified Approximately 60 Hours at $25^{\circ} \mathrm{C}$} \\
\hline Control $\ldots \ldots \ldots \ldots \ldots$ & $\ldots \ldots 198$ & 68.2 & 34.7 \\
\hline $\begin{array}{l}\text { Treated with carbobenzoxy- } \\
\text { chloride } \quad \ldots \ldots \ldots \ldots \ldots\end{array}$ & 39 & 3.5 & 2.3 \\
\hline $\begin{array}{l}\text { After catalytic removal } \\
\text { of carbobenzoxy groups }\end{array}$ & $\ldots \ldots 180$ & 40.4 & 23.8 \\
\hline \multicolumn{4}{|c|}{ Calcified Approximately 36 Hours at $25^{\circ} \mathrm{C}$} \\
\hline Control $\ldots \ldots \ldots \ldots \ldots$ & $\ldots \ldots 242$ & 26.5 & 14.9 \\
\hline $\begin{array}{l}\text { Treated with carbobenzoxy- } \\
\text { chloride } \ldots \ldots \ldots \ldots \ldots \\
\text { After catalytic removal of }\end{array}$ & $\ldots \ldots$ & 1.3 & 0.65 \\
\hline carbobenzoxy groups ... & $\ldots \ldots$ & 5.5 & 1.8 \\
\hline
\end{tabular}

lently bound to the mineral ions (presumably phosphate), and subject to the misgivings about the steric or electrostatic hindrance as noted earlier in interpreting the $\epsilon-\mathrm{NH}_{2}$ blocking experiments which showed the importance of such groups in in vitro calcification, we may interpret this to mean the interaction between the vast majority (95 per cent or more) of the $\epsilon-\mathrm{NH}_{2}$ groups and mineral ions is electrostatic. This does not rule out the possibility that the initial event, particularly in vivo, is a phosphorylation of collagen to form either -N-P-alkyl amides or -O-P-ester linkages. Only a small number of such phosphate esters or amides need be formed to start the nucleation process, and the remaining phosphate ions as well as calcium ions could then simply be electrostatically attracted to the rest of the polar amino acid side chain groups of the collagen and to each other.

Further experiments are now in progress in which a number of other amino acid side-chain groups are being blocked singly and in combination, and in which not only the final result of nucleation (i.e., the amount of mineral formed) but also the binding of the various constituent mineral ions is being studied. These experiments should lead to a clearer understanding of both the exact roles of specific amino acids and mineral ions and the sequence of events leading up to nuclei formation. 
On the basis of the experimental evidence presented, it would appear that the collagens of all tissues which have fibrils in the specific macromolecular aggregation state characterized by the 640700 - $\AA$ axial repeat structure should be inherently capable of nucleating apatite crystals from metastable calcium phosphate solutions. In the case of the vertebrates, for example, the collagens of all the normally ummineralized tissues such as the skin, ligaments, and tendons are organized in this fashion, and yet under normal conditions only the collagens of skeletal system and tooth are mineralized; it is well known, however, that the former may become mineralized in pathological conditions. To explain these findings, one might suppose that bone, cartilage, and tooth collagens are somehow different from the collagens of non-mineralized connective tissues. This, however, would not explain how ligaments, tendons, or skin collagens, for example, may be calcified pathologically.

On the other hand, it seemed more reasonable to us to postulate that in normally unmineralized tissues there are other substances present which under ordinary circumstances prevent or inhibit calcification (Glimcher et al., 1957; Glimcher, 1958, 1959). Data indicating that the latter is the correct interpretation come from both in vitro and in vivo studies. In vitro experiments conducted in our laboratories have shown that, whereas native (as opposed to reconstituted) collagen of tissues such as rat-tail tendon, guinea-pig skin, calfskin, etc., were not able to mineralize, these same collagen fibrils were able to mineralize after appropriate treatment of the tissue by procedures designed to extract and depolymerize certain components of the ground substance (Glimcher, 1958, 1959; Fig. 13). This, coupled with the fact that the collagen from these normally unmineralized tissues, when extracted, purified, and reconstituted into native-type fibrils essentially free of ground substance, is able to mineralize, appears to implicate other inhibitory substances in such tissues.

Very strong additional support for the hypothesis that nativetype collagen fibrils are inherently able to mineralize comes from the studies of Likens et al. (1958), Nylen et al. (1958), and Johnson (1958), who showed by chemical as well as by electron microscope and $\mathrm{x}$-ray microscope methods that the original collagen fibrils of turkey tendons, and not a special kind of bone collagen, initially calcified in vivo. 
It should be clearly kept in mind that what we have been discussing is the physicochemical basis of the induction of mineralization. The mineralization of the endoskeleton of vertebrates and the exoskeleton of certain invertebrates is a vital process which demands exquisite homeostatic control mechanisms which are highly specific in their operation. As in so many other cases in the organism where

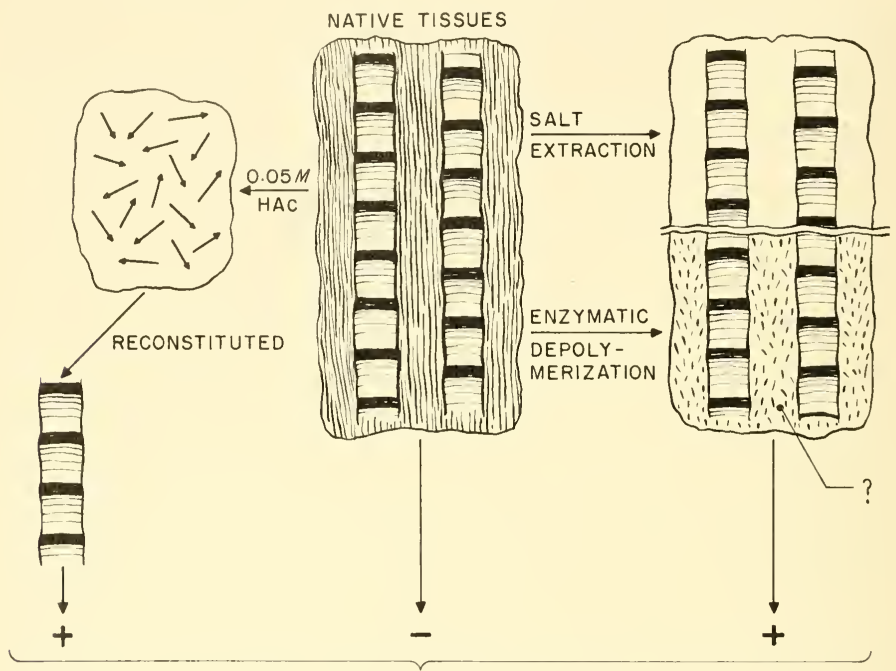

CALCIFICATION

Fig. 13. Diagrammatic illustration of the possible role of certain components of the ground substance (long threadlike material surrounding the collagen fibrils) in helping to inhibit calcification in normally unmineralized tissues. The "?" indicates that it is not yet certain whether depolymerization (represented by the short segments surrounding the fibrils) is itself effective, or whether depolymerization and subsequent removal from the tissue are necessary. (From Glimcher, 1958.)

control and specificity must be of the highest order, in both normally mineralized and normally unmineralized tissues a number of highly specific regulating mechanisms obviously must exist. There is every reason to expect that mineralization in both kinds of tissues is under the control of many delicately balanced regulatory factors, providing the organismic and cellular regulation of the mechanism which initiates the formation of the inorganic crystals and which controls their further growth. 


\section{References}

Block, R. J. 1951. Chemical classification of keratins. Ann. N. Y. Acad. Sci. 53: 608.

Block, R. J., M. K. Horwitt, and D. Bolling. 1949. Composition of protein of human teeth and fish scales. J. Dental Research 28: 518.

Carlstron, D., A. Engstrom, and J. B. Finean. 1955. The influence of collagen on the organization of apatite crystallites in bone. Symposia Soc. Exptl. Biol. 9: 85 .

Carlstrom, D., and J. B. Finean. 1954. X-ray diffraction studies on ultrastructure of bone. Biochim. et Biophys. Acta 13: 183.

Cartier, P. 1951. Biochimie de l'ossification. La Role de l'acide adenosine triphosphorique dans la minéralization du cartilage ossifiable. J. physiol. (Paris) 43: 677.

Cartier, P. 1952. Le Méchanisme enzymatique de l'ossification. Exposés ann. biochim. méd. 14:73.

Cartier, P. 1959. La Minéralization du cartilage ossifiable. VIII. Les Pyrophosphates du tissu osseux. Bull. soc. chim. biol. 41: 573.

Cartier, P., and J. Picard. 1955. La Minéralization du cartilage ossifiable. III. Le Méchanisme de la reaction atpasique du cartilage. Bull. soc. chim. biol. 37: 1159.

DiStefano, V., W. F. Neuman, and G. Rouser. 1953. The isolation of a phosphate ester from caicifiable cartilage. Arch. Biochem. Biophys. 47: 218.

Engel, M. B., N. R. Joseph, and H. R. Catchpole. 1953. Equilibrium of calcium and other ions in connective tissues. 5th Conf. on Metabolic Interrelations, Trans. Josiah Macy, Jr., Foundation Publications, New York. P. 105.

Finean, J. B., and A. Engstrom. 1953. Low-angle x-ray diffraction of bone. Nature 171: 564 .

Fitton-Jackson, S. 1957. The structure of developing bone in the embryonic fowl. Proc. Roy. Soc. (London) B 146: 270.

Freudenberg, E., and P. Gyorgy. 1921. Ueber Kalkbindung durch Tierische Gewebe III. Biochem. Z. 118: 50.

Gersh, I. 1952. Some functional considerations of ground substance of connective tissues. 2nd Conf. on Connective Tissues, Trans. Josiah Macy, Jr., Foundation Publications, New York. P. 11.

Glimcher, M. J. 1958. The specificity of the molecular structure of organic matrices in mineralization. In li. Sognnaes (ed.). Symposium on Calcification in Biological Systems, Washington, D. C., Dec., 1958. Am. Assoc. Advance. Sci. P. 421.

Glimcher, M. J. 1959. The molecular biology of the mineralized tissues with particular reference to bone. Revs. Modern Phys. 31: 359.

Glimcher, M. J., A. J. Hodge, and F. O. Schmitt. 1957. Macromolecular aggregation states in relation to mineralization: The collagen hydroxyapatite system as studied in vitro. Proc. Nat. Acad. Sci. U. S. 43: 860.

Glimcher, M. J., A. J. Hodge, and F. O. Schmitt. 1960a. Electron optical and $\mathrm{x}$-ray diffraction studies of bone and in vitro calcified collagens. (In press.)

Glimcher, M. J., A. J. Hodge, and F. O. Schmitt. 1960b. The specificity of the macromolecular aggregation state of collagen in in vitro calcification. (In press.)

Gregoire, C. J. 1957. Topography of the organic components in mother-of-pearl. J. Biophys. Biochem. Cytol. 3: 797.

Gross, J., and B. Dumsha. 1958. Elastoidin: A two-component member of the collagen class. Biochim. et Biophys. Acta 28: 268.

Gross, J., J. H. Highberger, and F. O. Schmitt. 1954. Collagen structures considered as states of aggregation of a kinetic unit: The tropocollagen particle. Proc. Nat. Acad. Sci. U. S. 40:697.

Gross, J., J. H. Highberger, and F. O. Schmitt. 1955. Extraction of collagen from connective tissues by neutral salt solutions. Proc. Nat. Acad. Sci. U. S. 41: 1. 
Gutman, A. B., and T. F. YU. 1950. Further studies of the relation between glycogenolysis and calcification in cartilage. 1st Conf. on Metabolic Interrelations, Trans. Josiah Macy, Jr., Foundation Publications, New York. P. 11.

Highberger, J. H., J. Gross, and F. O. Schmitt. 1951. The interaction of mucoprotein with soluble collagen; an electron microscope study. Proc. Nat. Acad. Sci. U. S. 37: 286.

Johnson, L. C. 1958. Mineralization of turkey leg tendon. I. Histology and histochemistry of mineralization. In R. Sognnaes (ed.). Calcification in Biological Systems, a Symposium presented at the Washington Meeting of the American Association for the Advancement of Science, Dec. 29, 1958. Publication Number 64 of the American Association for the Advancement of Science, Washington, D. C., 1960. P. 117.

Likens, R. C., K. A. Piez, and M. L. Kunde. 1958. Mineralization of turkey leg tendon. III. Chemical nature of the protein and mineral phases. In R. Sognnaes (ed.). Calcification in Biological Systems, a Symposium presented at the Washington Meeting of the American Association for the Advancement of Science, Dec. 29, 1958. Publication Number 64 of the American Association for the Advancement of Science. Washington, D. C., 1960. P. 143.

McLean, F. C. 1958. Phosphorus Metabolism. In K. Rodahl et al., (eds.). Bone as a Tissue, Proceedings of a Conference held at The Lankenau Hospital, Philadelphia, Pa., October 30-31, 1958. McGraw-Hill Book Co., Inc., New York, 1960.

Neuman, W. F. 1950. Bone as a problem in surface chemistry. 2d Conf. on Metabolic Interrelations, Trans. Josiah Macy, Jr., Foundation Publications, New York. P. 32 .

Neunan, W. F., and M. W. Neuman. 1953. The nature of the mineral phase of bone. Chem. Revs. 53: 1.

Nylen, M. U., D. B. Scott, and V. M. Mosley. 1958. Mineralization of turkey leg tendon. II. Collagen-mineral relations revealed by electron and $\mathrm{x}$-ray microscopy. In R. Sognnaes, (ed.). Calcification in Biological System, a Symposium presented at the Washington meeting of the American Association for the Advancement of Science, Dec. 29, 1958. Publication Number 64 of the American Association for the Advancement of Science, Washington, D. C., 1960. P. 129.

Olcott, H. S., and H. Fraenkel-Conrat. 1947. Specific group reagents for proteins. Chem. Revs. 41: 151.

Polonovski, M., and P. Cartier. 1951. Sur le premier stade biochemique de l'ossification. Compt. rend. 232: 119.

Putnam, F. 1953. The chemical modification of proteins. In H. Neurath and K. Bailey (eds.). The Proteins. Academic Press, Inc., New York. Vol. I, part B, p. 893 .

Rich, A., and F. H. C. Crick. 1955. The structure of collagen. Nature 176: 915.

Rich, A., and F. H. C. Crick. 1958. The structure of collagen. In G. Stainsby (ed.). Recent Advances in Gelatin and Glue Research. Pergamon Press, Ltd., London. P, 20.

Robinson, R. A. 1952. An electron-microscopic study of the crystalline inorganic component of bone and its relationship to the organic matrix. J. Bone and Joint Surg. 34A: 389.

Robinson, R. A., and M. L. WAtson. 1952. Collagen-crystal relationships in bone as seen in the electron microscope. Anat. Record 114: 383.

Robinson, R. A., and M. L. WAtson. 1955. Crystal-collagen relationships in bone as observed in the electron microscope. III. Crystal and collagen morphology as a function of age. Ann. N. Y. Acad. Sci, 60: 596.

Rubin, P. S., and J. E. Howard. 1951. Histochemical studies in the role of acid mucopolysaccharides in calcifiability and calcification. $2 d$ Conf. on Metabolic Interrelations, Trans. Josiah Macy, Jr., Foundation Publications, New York. P. 155.

Schмint, W. J. 1935. Polarisationsoptische Analyse des submikroskopischen Baues von Sellen und Geweben. Handbuch biol. Arbeitsmethoden Abt. 5, T10, p. 435. 
Schmitt, F. O., J. Gross, and J. H. Highberger. 1955a. Tropocollagen and the properties of fibrous collagen. Exptl. Cell Research 3 (Suppl.): 326.

Schntt, F. O., J. Gross, and J. H. Highberger. 1955b. States of aggregation of collagen. In Fibrous Proteins and Their Biological Significance. Symposia Soc. Exptl. Biol. 9: 148.

Schmitt, F. O., C. E. Hall, and M. A. Jakus. 1942. Electron microscope investigations of the structure of collagen. J. Cellular Comp. Physiol. 20: 11.

Sobel, A. E. 1955. Local factors in the mechanism of calcification. Ann. N. Y. Acad. Sci. 60: 713 .

Sobel, A. E., and M. Burger. 1954. Calcification. XIV. Investigation of the role of chondroitin sulfate in the calcifying mechanism. Proc. Soc. Exptl. Biol. Med. 87: 7.

Solomons, C. C., and J. T. Inving. 1958. The reactions of some hard- and soft-tissue collagens with 1-fluoro-2:4-dinitrobenzene. Biochem. J. 68: 499.

Speckman, T. WV., and W. P. Norris. 1957. Bone crystallites as observed by the electron microscope. Science 126: 753.

Strates, B. S., IV. F. Neuman, and G. J. Levinskas. 1957. The solubility of bone mineral. II. Precipitation of near-neutral solutions of calcium phosphate. J. Phys. Chem. 61: 279.

Strates, B., and W. F. Neuman. 1958. On the mechanism of calcification. Proc. Soc. Exptl. Biol. Med. 97: 688.

Tsuji, T., D. G. Sharp, and K. M. Wilbur. 1958. Studies on shell formation. VIl. The submicroscopic structure of the shell of the oyster Crassostrea virginica. J. Biophys. Biochem. Cytol. 4: 275.

Turnbull, D. J. 1950a. Kinetics of heterogeneous nucleation. J. Chem. Phys. 18: 198.

Turnbull, D. J. 1950b. Formation of crystal nuclei in liquid metals. J. Appl. Phys. 21: 1022.

Turnbull, D. J. 1952. Kinetics of solidification of supercooled liquid mercury droplets. J. Chem. Phys, 20: 411.

Turnbull, D. 1956. Phase changes. Advances in Solid State Physics 3: 225.

Turnbull, D., and B. Vonnegut. 1952. Nucleation catalysis. Ind. Eng. Chem. 44: 1292.

Underwood; D. L., and H. J. White, JR. 1954. The absorption of sodium sulphate and sulphuric acid by hair. In The Physical Chemistry of Dyeing and Tanning. Discussions Faraday Soc. No. 16: 66.

Watabe, N., D. G. Sharp, and K. M. Wilbur. 1958. Studies on shell formation. VIII. Electron microscopy of crystal growth of the nacreous layer of the oyster Crassostrea virginica. J. Biophys. Biochem. Cytol. 4: 281.

Zaмiвотті, V. 1957. The biochemistry of preosseous cartilage and of ossification. Sci. Med. Ital. 5: 614 .

\section{DISCUSSION}

\section{K. E. Van Holde, M. J. Glimcher, J. W. Green, S. B. Barker, L. V. Beck, C. L. Prosser, A. G. Richards}

Dr. VAN Holde (University of Illinois): Have you studied the binding of phosphate ion by collagen fibrils or by soluble collagen?

Dr. Glimcher: We are currently studying the binding of both calcium and phosphorus by collagen macromolecules in solution and by the "solid" collagen fibrils in small decalcified bone particles and plan to repeat the studies using purified reconstituted collagens as well as collagen in solution. In these studies we have encountered several difficult technical problems, as well as some interesting phenomena which are difficult to interpret. In the first place, only 
a relatively small amount of the phosphorus is bound by the collagen, making quantitative determinations difficult. Secondly, the amount of phosphorusbinding is so small that the correction for the amount of phosphorus in the solvent water of the fibrils is proportionately large. Since there is no way as yet of determining accurately the amount of "bound" or "free" water in the fibrils, we have been unable to correct for this factor quantitatively. Thirdly, part of the phosphorus appears to be irreversibly bound to the collagen; in fact, there may be a spectrum of phosphorus-binding with different binding energies, and when this is coupled with diffusion phenomena, the final results are as yet impossible to interpret. Similar difficulties have been encountered by workers using $\mathrm{S}^{35}$ to study the sulfate-binding of solid keratin structures such as hair (Underwood and White, 1954).

In relation to the mechanism of nucleation, when phosphate-binding was measured in solutions the concentrations of which were similar to those used in calcifying solutions (calcium and phosphate solutions), the amount of phosphate bound was very small compared to the amount of phosphorus bound or otherwise adsorbed in the initial stages of nucleation before detectable amounts of crystallization had occurred. This has suggested that the "building up" of the nucleus is not a simple addition of phosphate and calcium ions independent of each other, but rather a cooperative phenomenon in which the calcium ions, phosphate ions, and certain amino acids interact collectively to influence the state of aggregation of the mineral ions. To this must be added the possibility that specific clusters of calcium and phosphate ions are the initially bound moiety.

Dr. Green (Rutgers University): Have you examined the collagens in elasmobranch fishes? Have you any information bearing on the failure of these collagens to be mineralized?

Dr. Glimcher: We have only done a few experiments with the so-called elastoidin of shark fins. As you know, this collagen has some of the low-angle, as well as the wide-angle, $\mathrm{x}$-ray diffraction properties of other native collagens, but is cross-linked and very insoluble. The tissues also contain another as-yetunidentified protein (Gross and Dumsha, 1958). Since we were not able to get the elastoidin free of other constituents, or to solubilize and reconstitute the fibrils, we were unable to investigate this system adequately.

I can only speculate as to why such tissues are not normally mineralized. We do not know whether, in fact, the collagen in elastoidin has the same molecular structure and macromolecular aggregation state as other native collagens. According to the concept which I presented today, however, if the structural characteristics were the same, one would have to investigate: (1) the metastability of the extracellular fluids; (2) the possibility of the presence of inhibitory substances; and (3) the lack of regulatory mechanisms, presumably enzyme-controlled, which are probably needed to initiate the nucleation process in vivo. We could presumably get some useful information about these questions if it were possible in vitro to test the mineralizing capacity of both the native tissue and the purified elastoidin in a fashion similar to the native collagen-rich tissues, and of the purified collagen from these tissues in a fashion similar to the experiments which I have already described. 
Dr. Barker (University of Alabama Medical Center): Should we not be as critical of FDNB's blocking free amino groups other than $\epsilon$-amino groups of lysine or hydroxylysine of reconstituted collagen as we are of the lack of specific freeing of $\epsilon$-amino groups upon decalcification of bony structures?

Dr. Glimcher: I would be the first to agree that we must be extremely cautious in interpreting any group-blocking experiments, particularly if only one blocking agent such as FDNB is used. Because of the extremely large size of the collagen macromolecule, the number of alpha amino groups is relatively small; thus, we can be reasonably sure that the major effect of using FDNB is to block the $\epsilon-\mathrm{NH}_{2}$ groups. As I pointed out, however, it is quite possible that the actual sites involved in nucleation are adjacent to the $\epsilon-\mathrm{NH}_{2}$ groups, and that when one blocks the $\epsilon-\mathrm{NH}_{2}$ groups by substituting such large radicals as FDNB, the really important adjacent groups are sterically (or electrostatically) shielded and prevented from interacting with the mineral ions. We would then be misled and erroneously assign a role to the $\epsilon-\mathrm{NH}_{2}$ groups. It is for these reasons, and not the lack of the specificity of FDNB (and other blocking agents), that I am most concerned about in the interpretation of the results of experiments such as the ones that I described.

Dr. BECK (University of Pittsburgh): Is it too early to speculate on the role(s) of parathormone, Vitamin D, and dihydroergosterol?

Dr. Glimcher: Yes!

Dr. Prosser (University of Illinois): Is there any correspondence between apatite crystal formation in bone and calcification of chitin in crustaceans? Also calcification in clams, where the protein does not show the periodicity of collagen?

Dr. Gumcher: Although we have only studied the collagen hydroxyapatite system in vitro, we believe that the underlying basic principle, wherein the organic matrix nucleates the inorganic crystals as a result of a specific stereochemical array of certain reactive groups, may apply to biological mineralization in general. In the case of oyster shells, for instance, calcium carbonate in the form of either calcite or argonite is deposited in an intimate relationship to the organic matrix, conchiolin, which is composed of conchiolin, an unidentified protein. The fact that there is no repeating structure visible with the electron microscope in the organic matrix of such material does not mean that the matrix is not a well-organized and well-ordered structure at the molecular level. In the case of collagen we are fortunate in being able to "see" this ordering by electron microscopy and by low-angle $\mathrm{x}$-ray diffraction because of certain characteristics dependent on the periodic concentration of certain reactive polar amino acids.

Dr. Richards (University of Minnesota): Do the apatite microcrystals in bone have the typical crystal faces of larger crystals grown from solution in vitro? If so, do you have any idea why in some cases, such as the subcutaneous anchors of holothurian worms, one finds normal calcite crystals in weird shapes such as anchors?

Dr. Glimcher: Unfortunately, the apatite crystals of bone are so small $(15-30 \AA \times 200-400 \AA)$ that it is impossible to be sure either of their gross external habit or of their individual crystal faces. 
Neither the physical nor the biological factors controlling crystal growth are well enough known to answer the second part of your question. It may be of interest, however, that the apatite crystals in the enamel of tooth are very much larger than those in the adjacent dentin. Presumably, part of this may be due to rates of crystal growth; i.e., the slower the rate, the larger and more perfect the crystals. I have been told that the "spikes" of certain sea urchins which are 7 inches or longer are composed of a single crystal of calcite. 


\title{
The Chloroplast: Its Lamellar Structure
}

\section{and Molecular Organization ${ }^{1}$}

\author{
J. J. Wolken ${ }^{2}$
}

A basic problem in biology is the conversion and transfer of energy, e.g., light energy to chemical energy or to electrical energy. Recently, research workers have been investigating how the chloroplast, a differentiated cytoplasmic structure of plant cells, efficiently performs this conversion in the process of photosynthesis. The number of research publications, reviews, and symposia in the past few years on the chloroplast structure and energy-transfer are evidence of interest in this exciting problem (Boulder Conference on Biophysics, 1959; Brookhaven Symposia, 1958; Frey-Wyssling, 1957; Gaffron, 1957; Granick, 1955; Mühlethaler, 1955; Thomas, 1955, 1958; Wolken, 1959). The purpose, then, of this discussion is to describe the structure and composition of the chloroplast as revealed by microscope studies and chemical analyses, and from the structure and composition to suggest a model for the pigment molecules within the chloroplast; then, if possible, from such a model to speculate about the molecular structure and function of the chloroplast in energy transfer.

The photoreceptors of plant cells exist in a variety of shapes and sizes, from those of the photosynthetic bacteria to those of the higher plants. These structures have been given a varied nomenclature, i.e., chromatophores, megaplasts, plastids, and grana, depending on their phylogenetic position and internal organization. Because of inconsistencies in these definitions, even additional nomenclature has been suggested. All of these structures except

${ }^{1}$ Aided in part by grants from U. S. P. H. S. Institute Neurological Diseases and Blindness (B-397 C5), National Council to Combat Blindness (G-199 C7), and the McClintic Endowment.

${ }^{2}$ Biophysical Research Laboratory, Eye and Ear Hospital, and the University of Pittsburgh Medical School, Pittsburgh, Pennsylvania. 
those in which the photosynthetic pigments are located in small granules, chromatophores, will be here referred to as chloroplasts.

It is technically difficult to isolate chloroplasts from other cellular components, and precise data on their chemical composition are not easily obtainable. A summary of the data on isolated chloroplasts

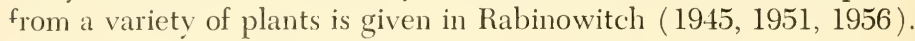
In all of these studies of composition, particularly of the pigments and their concentration, the state of the plant cells, age, nutrition, temperature, and light conditions are extremely important.

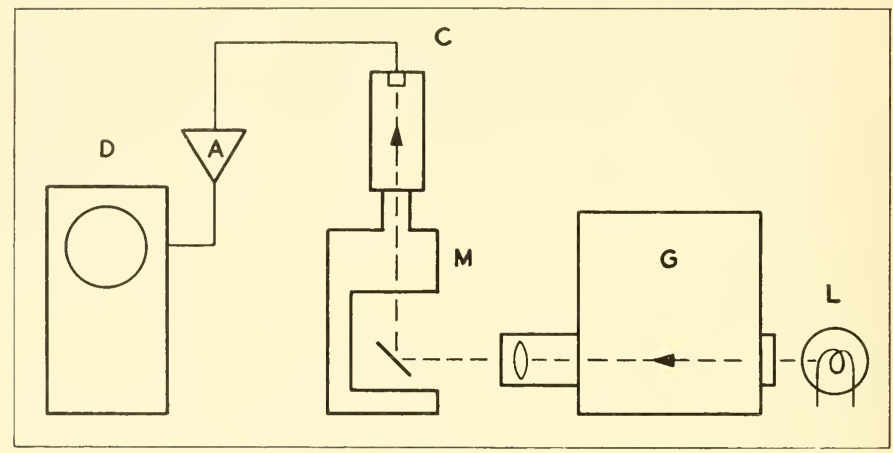

Fig. 1. Diagram of microspectrophotometer. L, light source; G, monochromator; $M$, microscope with reflecting optics; $C$, photoconductive cell; $A$, amplifier; $D$, oscilloscope.

A range of values on a dry-weight basis from 35 to 55 per cent for the protein, 18 to 37 per cent for the lipids, and 5 to 8 per cent for the inorganic materials is given for a variety of plant chloroplasts. Studies are being made to determine the kinds of proteins and their amino acid composition (Sissakian, 1958). Two cytochromes, cytochrome $f$ and cytochrome $b_{3}$, together, can make up as much as 20 per cent of the chloroplast's total protein content. Nucleic acids (both RNA and DNA) are also reported to be in the chloroplasts; experimental values range from 0.3 to 3.5 per cent on a dry-weight basis.

The chlorophyll pigments constitute about 5 to 8 per cent of the chloroplast (although concentrations as high as 20 per cent have been reported), and the carotenoids are about 2 per cent. Chlorophyll a occurs in all plant chloroplasts, but other isomers are also 
found; in the higher plants, chlorophylls $a$ and $b$ occur in a ratio of about 3:1. The number of chlorophyll molecules per chloroplast is of the order of $10^{9}$. The carotenoids, the xanthophylls, particularly lutein and zeaxanthin, and the carotene, $\beta$-carotene, are intimately

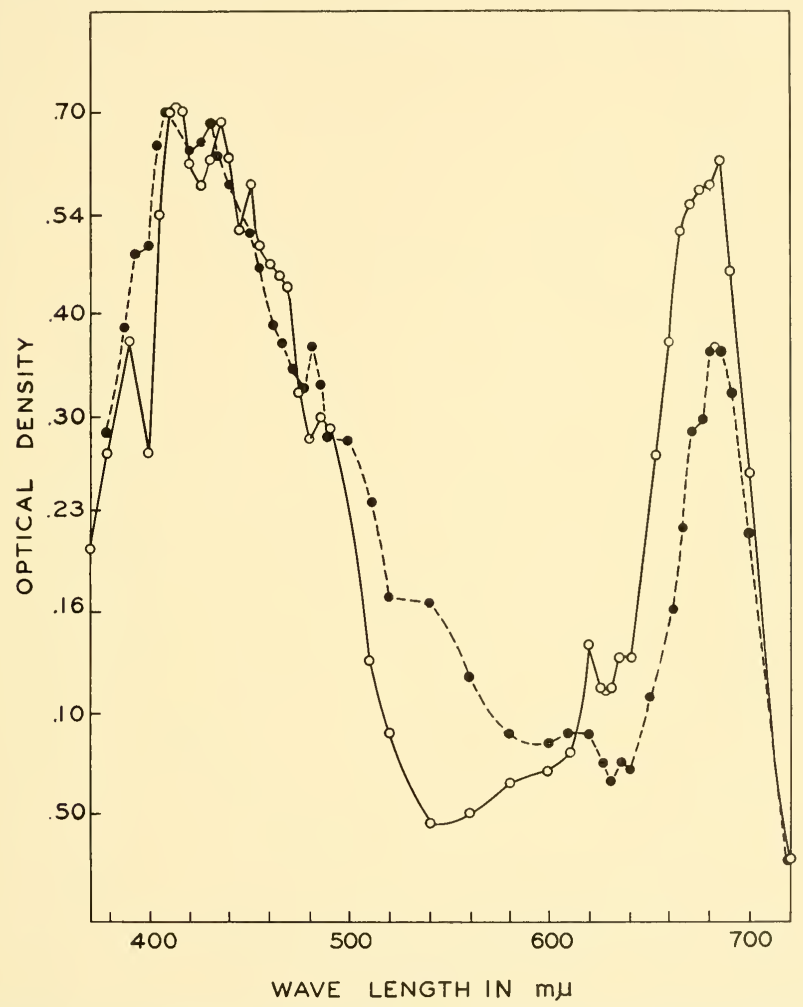

Fig. 2. In vivo spectra from two different Euglena chloroplasts taken with the microspectrophotometer $\left(8 \mu^{2}\right.$ area).

associated with chlorophyll in the chloroplast. Other photosynthetic pigments, phycoerythrin and phycocyanin, are present in the red and blue-green algae.

In order to analyze more directly the kinds of pigments and their concentrations in a single chloroplast, we have designed and built a 
microspectrophotometer (Strother and Wolken, 1959). The photosensitive element is a cadmium selenide photoconductive cell with a light-sensitive surface measuring approximately $0.5 \mathrm{~mm} \times 1 \mathrm{~mm}$. The photocell output is amplified by a transistorized dc amplifier

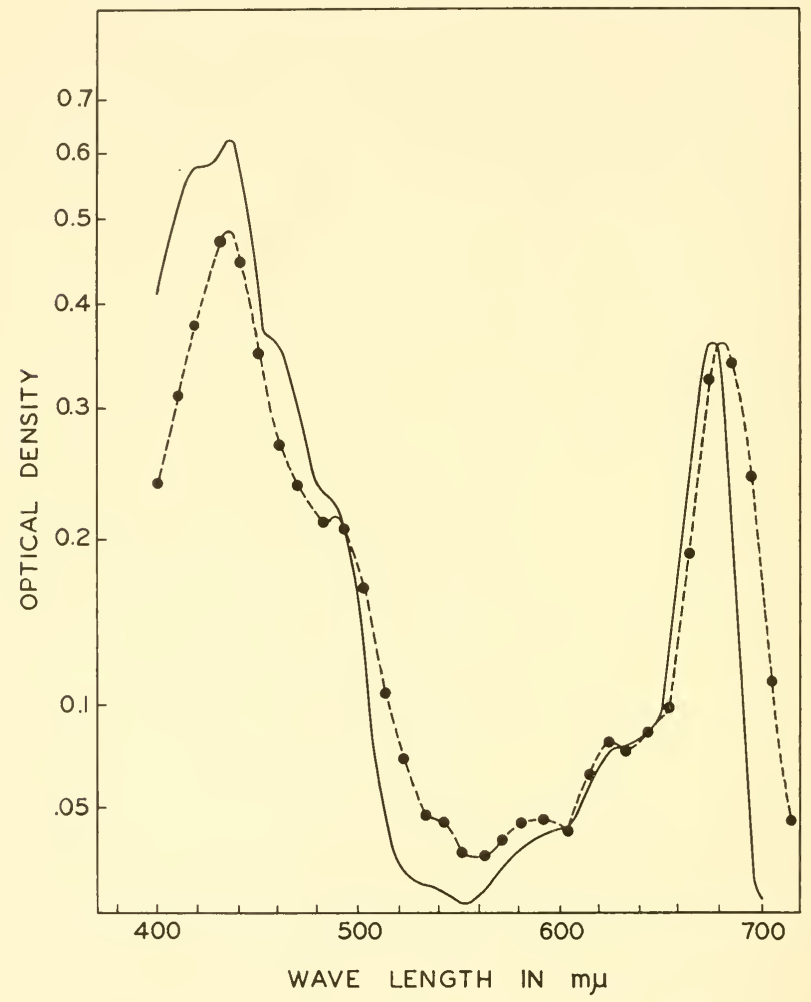

Fig. 3. Spectrum of Euglena chloroplastin (digitonin extract of chloroplasts), from Beckman DK-1 recording spectrophotometer, compared to spectrum of Euglena chloroplast taken with microspectrophotometer. plast spectrum. chloroplastin spectrum.

and displayed on an oscilloscope used as a de voltmeter. A grating monochromator is used for illumination, and the microscope is fitted with reflecting optics (Fig. 1). Absorption spectra are obtained by plotting readings of the dc output of the photocell at selected wave 
lengths. The instrument, with quartz components and a xenon arc light source, can be used over the wave-length range 200 to 990 $\mathrm{m} \mu$ at a low magnification for specimen areas of $16 \mu^{2}$ to $2 \mu^{2}$, at an entering half-band width of $2.6 \mathrm{~m} \mu$. In Fig. 2 are shown the absorption spectra obtained with this instrument for the chloroplasts

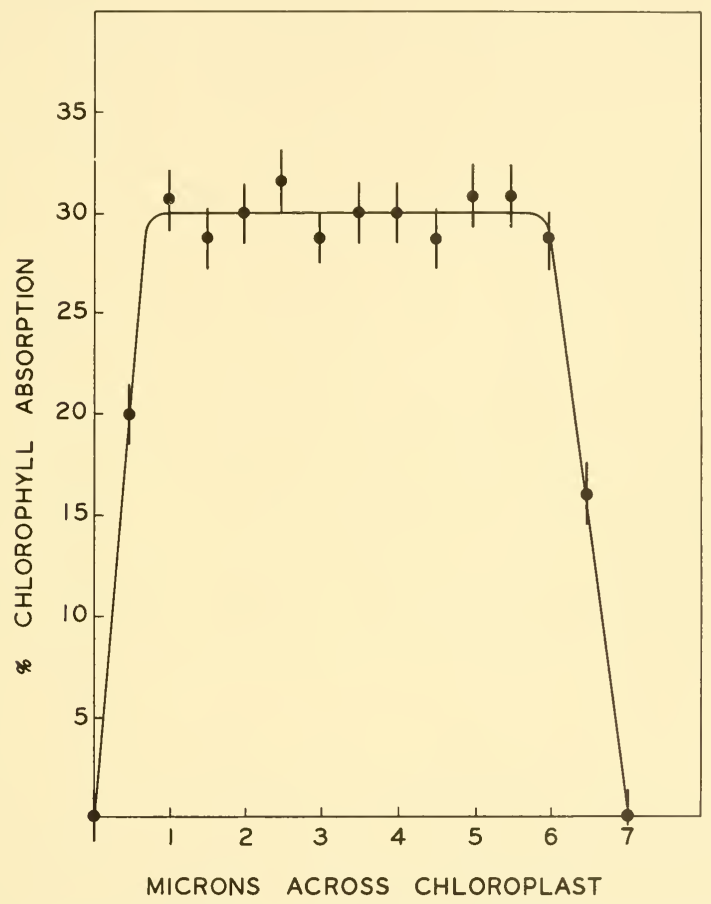

Fig. 4. Absorption across a single chloroplast at $0.5 \mu$ intervals as measured at $675 \mathrm{~m} \mu$ relative to $550 \mathrm{~m} \mu$ with the microspectrophotometer, indicating the distribution of chlorophyll in the chloroplast.

in two different euglenas. The magnification was $250 \times$; an area of $8 \mu^{2}$ is represented. These spectra begin to show fine structure. The major absorption peaks are near 675 and $435 \mathrm{~m} \mu$, with other peaks at 480,590 , and $625 \mathrm{~m} \mu$. Since these are in vivo spectra, some shifts are to be anticipated from the spectrum of the pigments dissolved in organic solvents. These spectra (Fig. 2) closely approximate 
previously obtained spectra for the in vivo Euglena chloroplast and for digitonin extracts of their chloroplasts (Fig. 3). The concentration of chlorophyll calculated from the absorption data corroborates the previous analysis that there are of the order of $10^{9}$ chlorophyll molecules per chloroplast. By scanning across the chloroplast at $0.5 \mu$ intervals at the absorption maximum $675 \mathrm{~m} \mu$ and at the absorption minimum $5.50 \mathrm{~m} \mu$, it can be shown that the chlorophyll is evenly distributed throughout the whole chloroplast (Fig. 4). Because of the limitation in the resolving power of the instrument it is not possible to locate chlorophyll precisely in the chloroplast lamellae. Together with microspectrophotometry, the techniques of polarization, fluorescence, phase, and interference microscopy are the most direct approach for exploring cellular structure. These methods permit the study of the chloroplasts in their natural state.

The electron microscope now permits us to see through the fixed chloroplast in situ instead of seeing its shadow or replica. The increased resolution of the electron microscope has greatly aided our attempts to obtain a molecular picture of the chloroplast. In the preparation for electron microscopy, the cells were fixed with 1 per cent osmium tetroxide buffered with acetate-veronal $\mathrm{pH} 7$ to 8 . The osmotic changes were partially controlled by adding sucrose $(0.15 M)$ to the fixative. The fixed cells were embedded in a resin (n-butyl methacrylate, methyl methacrylate, or mixtures of these). Thin sections, less than $0.05 \mu$ in thickness, were cut with a glass knife.

\section{Chloroplast Structure}

There is now good evidence from a great variety of plant species that, although chloroplasts may differ in shape and size, they have in common a basic intemal organization. We have investigated the structure of chloroplasts in several algae and higher plants with the electron microscope. These studies reveal that the chloroplasts are composed of lamellae.

We have found the algal flagellate Euglena to be uniquely adaptable for experimental studies of the chloroplast structure, since Euglena behaves as a photosynthetic plant cell in the light and as an animal cell in the dark. Changes in the environmental conditions (light, darkness, temperature, drugs) are reflected in the organisms' chemistry and morphology. Using Euglena, we have been able to 
study the chloroplast by various microscope techniques and have attempted to correlate structural changes of the chloroplast with pigment synthesis and with photosynthesis in light $\leftrightarrow$ dark adaptation (Wolken and Palade, 1953; Wolken and Schwertz, 1953; Wolken et al., 1955; Wolken and Mellon, 1956; Wolken, 1956a ).

$E$. gracilis when light-grown contains many green elongated cylindrical chloroplasts 5 to $10 \mu$ in length and 0.5 to $2 \mu$ in diameter. When the chloroplasts are viewed by interference-phase, by absorption, and by polarizing microscopy, an ordered structure is indicated. Previously, it was proposed from studies of negative birefringence that the chloroplast is a lamellar structure of 20 to 30 lipid layers of the order of $50 \AA$, each separated from layers of aqueous protein of the order of $250 \AA$ by monomolecular films of chlorophyll molecules (Frey-Wyssling, 1957). There is now excellent proof of this lamellar structure from electron microscope studies, and x-ray diffraction techniques have corroborated the electron microscope observations, showing a repeating unit of the order of $250 \AA$.

In order to determine more precisely the lamellar structure and to test the pigment monolayer hypothesis, the diameter, length, number, and thickness of the lamellae of the chloroplasts were measured and statistically evaluated (Wolken and Schwertz, 1953). For example, the chloroplast of $E$. gracilis consists of 21 dense layers of the order of $250 \AA$ in thickness, with less dense interspaces of 300 to $500 \AA$ in thickness. Each dense layer appears to be covered on both sides by a thimer and denser layer (lamellae) of the order of 50 to $100 \AA$ in thickness. The average thickness measured for a variety of plant chloroplasts ranges from 20 to $100 \AA$ for the electron-dense lamellae (Leyon, 1956; Sager and Palade, 1957; Sager, 1958). FreyWyssling (1957) had noted in previous studies that the thin lamellae consist of spherical particles $65 \AA$ in diameter; these were considered to be the protein or lipoprotein macromolecules (Fig. 6).

There is experimental evidence to indicate that the chlorophyll molecules are preferentially oriented parallel to the lamellae and are within the very dense (lipoprotein) lamellae (Thomas, 1955). The electron-dense layers are believed to be lipoprotein and lipid material. The areas of least density are considered to be aqueous proteins, enzymes, and dissolved salts. From the geometry of the chloroplast, the number of dense layers, and the chlorophyll concentration per chloroplast (Table 1 ), we have calculated the crosssectional area occupied by each chlorophyll molecule to be $222 \AA^{2}$ 
for the Euglena chloroplast and $246 \AA^{2}$ for the Poteriochromonas chloroplast (Wolken and Schwertz, 1953). Elbers et al. (1957) have since collected data on chlorophyll concentrations and geometry of chloroplasts in a variety of plant species, and by similar analogy have calculated the mean area available for the chlorophyll molecule in the monolayer to be of the order of $200 \AA^{2}$. Studies of the dichroism, birefringence, and polarization of fluorescence in Mougeotia chloroplasts also indicate that chlorophyll resides as a monolayer on the lamellar surface, and the area available per chlorophyll molecule was calculated to be $\sim 250 \AA^{2}$ (Goedheer, 1955, 1957).

TABLE I

Geometry of Chloroplasts

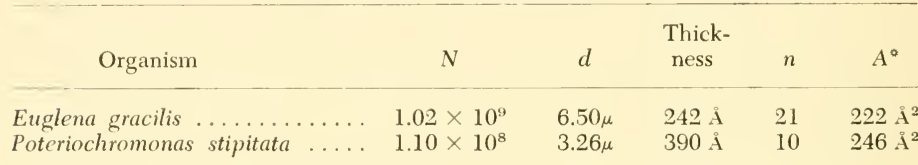

- $A$, area accessible to each chlorophyll molecule, is calculated from

$$
A=\frac{(8 / \pi) n d^{2}}{N},
$$

where $n$ is the average number of dense layers (double lamellae), providing $2 n$ surfaces for the pigment molecules, $d$ is the average observed length of the chloroplast, and $N$ is the average number of chlorophyll molecules per chloroplast (Wolken and Schwertz, 1953).

Since the cross-sectional area of the porphyrin head of the chlorophyll molecule is known from $\mathrm{x}$-ray studies to be about $225 \AA^{2}$ to $242 \AA^{2}$, these results indicate that all the available chlorophyll molecules could be packed into the interfacial area and cover all of the dense surface of the lamellae as a monolayer.

On the basis of these calculations, a simplified schematic molecular model was proposed (Fig. 5). The suggestion of Bass-Becking and Hanson (1937), that four chlorophyll molecules are united to form tetrads in which the reactive isocylic rings turn toward each other, was employed (Wolken and Schwertz, 1953). Interaction between the phytol tails was eliminated in the model by arranging the tetrads in such a way that one, and only one, of the phytol tails is located at each virtual intersection in the rectangular network. If the chlorophyll were packed as a monolayer as shown in the schematic molecular network in Fig. 5(b), there would still be space available 


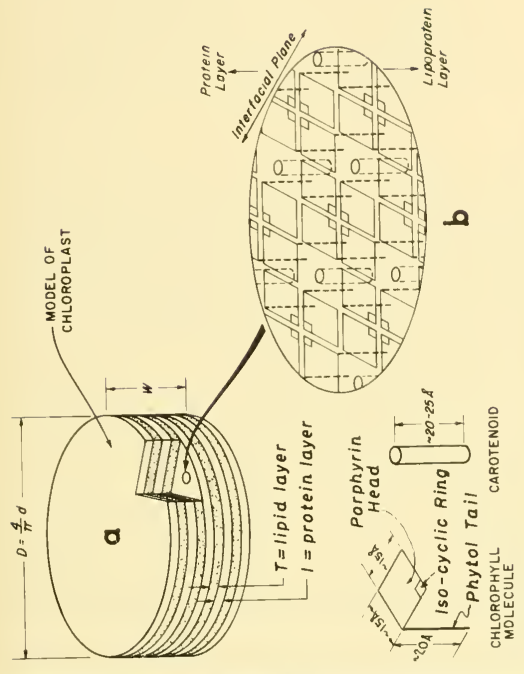

은 웜

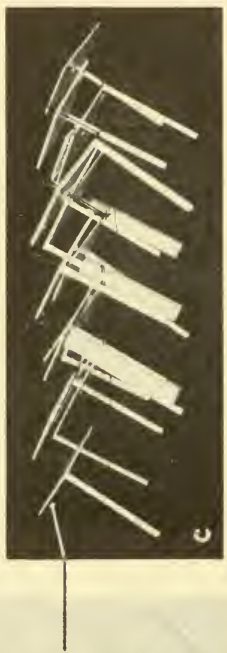

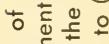

(1) E

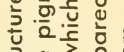

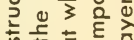

흔

능 등 응

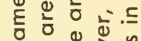

$\stackrel{\square}{\overline{0}} \stackrel{0}{0} \stackrel{0}{\frac{0}{0}}$

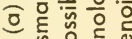

- 0 응

응 ㅇํㅇ

응 동

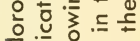

它 $\frac{5}{n}$ 을

ब 0 ठ

$\mp$ 岀

는ㅁㄴ응

ข

응

$\varepsilon \neq$ के

능

ว ․ㅡㄹ은

ญ 등 Ð

을 등

궁은

늠 은.

ह

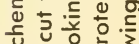

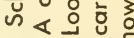

ம่อิ ๘

ตㄹ อ ธิ

计范市

응 충 

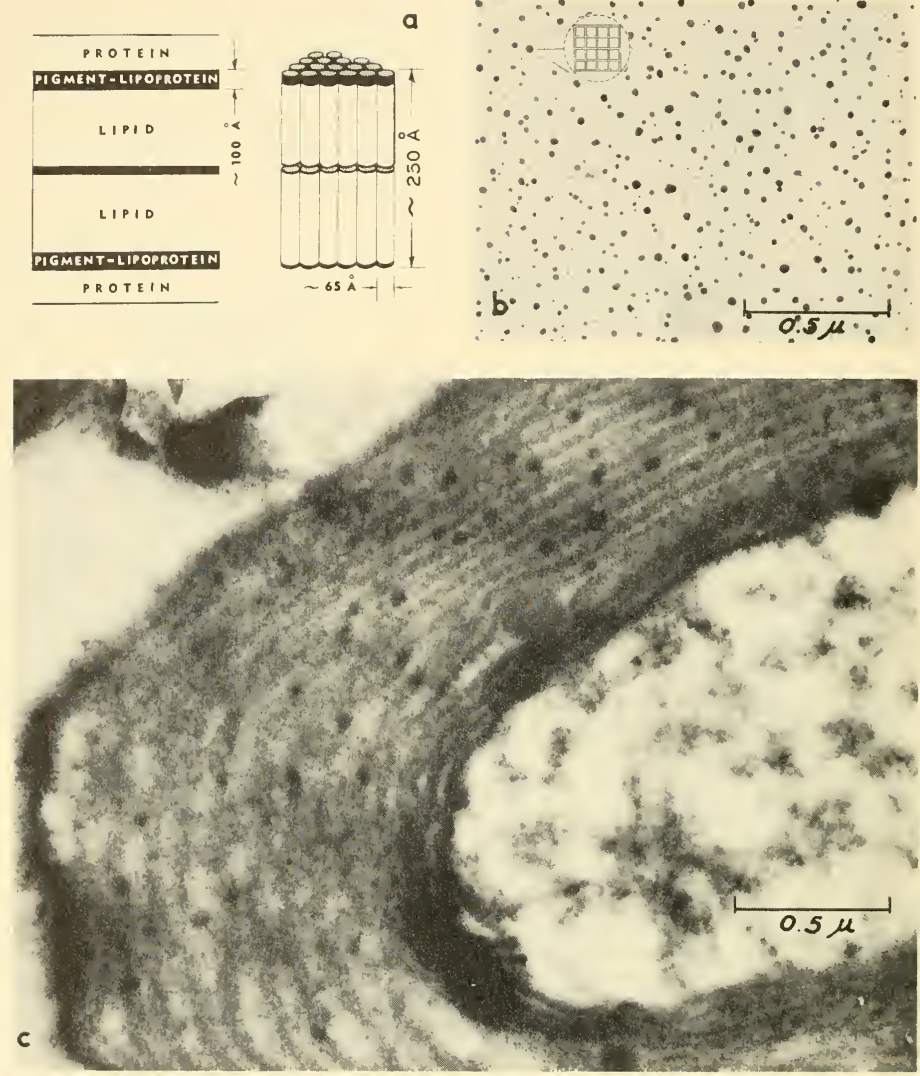

Fig. 6. (a) Schematic structural identification of the chloroplast layers. (b) Electron micrograph of chloroplastin, not fixed and not shadowed. (c) Electron micrograph of the chloroplast of Euglena granulata.

for the carotenoid molecules at the interstitial positions between the chlorophyll molecules. If these spaces are occupied as shown, there will be one carotenoid molecule for at least every three chlorophyll molecules in the network. This kind of close packing of the chlorophyll and carotenoid molecules in the pigment monolayers would also permit energetic interaction between them. Other models slightly modified from the one presented here indicate that 
the chlorophyll molecules are also turned inward, as well as being oriented on the surface (Hodge et al., 1955). Calvin has recently suggested a similar model in which the porphyrin heads lie at an angle of $45^{\circ}$; one protein layer has $\mathrm{CO}_{2}$-reducing enzymes and another protein layer has O-evolving enzymes (Calvin, 1958, 1959a, 1959 b).

There are several possible ways in which the chlorophyll molecules could be oriented in the lamellae. If the porphyrin heads of the chlorophyll molecules lay at $0^{\circ}$ as flat plates as indicated in Fig. 5 (b), their greatest cross-section would be available. However, if they were oriented within the lamellae at increasing angles up to $90^{\circ}$, the cross-sectional area available would be decreasing, as would the photosynthetic efficiency. Studies of chlorophyll monolayers on various liquid surfaces suggested that the chlorophyll molecules would probably lie at an angle of $35^{\circ}$ to $55^{\circ}$ within the chloroplast, thus reducing the above calculation for the cross-section of the chlorophyll molecule to $100 \AA^{-2}$ (Trurnit and Colmano, 1958). It is very likely that the absorption oscillators of these pigment molecules are arranged with an orderly orientation in a way that a maximum absorption will occur for an incident light polarized in a given direction.

The question of how the chloroplast evolves from a rudimentary proplastid into an organized structure is a difficult one to answer. Euglenas grown in darkness for long periods become colorless. We have noted that there is an obvious change in the chloroplasts; they fragment, and their lamellar structure is no longer recognizable. Such dark-adapted euglenas, brought back to the light and fixed for electron microscopy at regular time intervals, showed in electron micrographs, after as little as 4 hours' light exposure, recognizable elongated bodies with the characteristic laminations of chloroplasts. At the beginning, their lamellae were very thin and few in number and were not tightly or regularly packed. The lamellae grew in size and number progressively with continuing light exposure along with the increase in the synthesis of chlorophyll, and, by 72 hours of light exposure, the chloroplasts had the form and organization described for actively photosynthesizing euglenas (Wolken and Palade, 1953). As soon as we could detect chlorophyll spectrophotometrically, the chloroplasts were already lamellar. The alga Chlamydomonas, whether grown in light or in darkness, shows a chloroplast organization of lamellae, pyrenoid, and eyespot within a limiting 

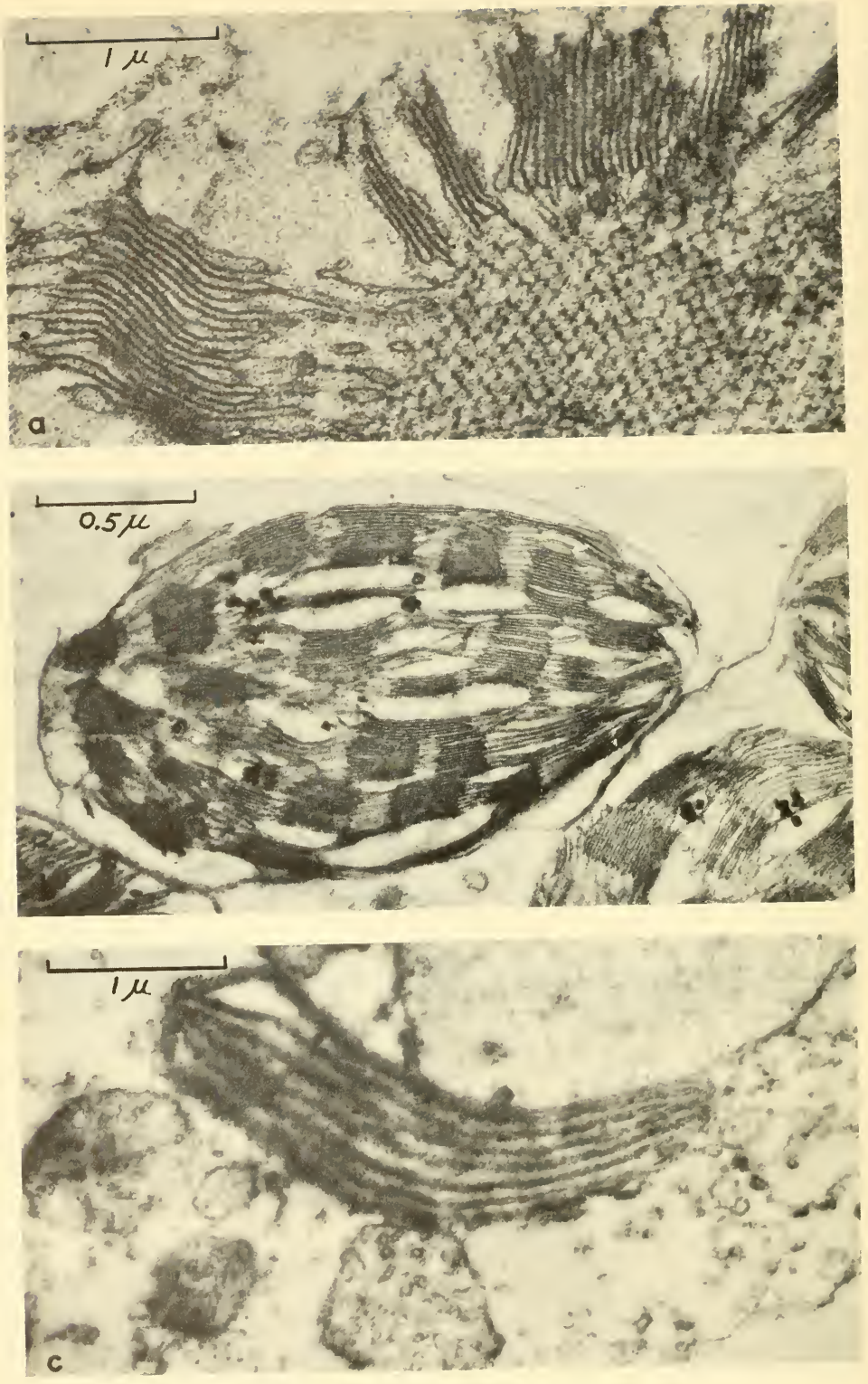

Fig. 7. (See next page for caption.) 
membrane. In the absence of chlorophyll, the lamellae are not formed, although the eyespot, starch grains, and pyrenoid are still found within the chloroplast membrane (Sager and Palade, 1957; Sager, 1958).

In the development of the chloroplast in flowering plants, the first visible stages lack all the structural properties typical of the fully developed chloroplast. Heitz (1955) and Leyon (1956) conclude from electron microscope studies that there is a primary granum that has a crystalline structure. Whether such crystalline bodies exist in the living state or whether their crystallinity is enhanced by preparative techniques for electron microscopy is difficult to decide. The crystalline structure of the primary granum, as found in Chlorophytum, Aspidistra, and other monocotyledons, is also present in dicotyledons. This suggests that there must exist some association between the crystalline lattice and the development of the lamellae (Heitz, 1955). Miihlethaler (1959) has shown that the lamellar structure develops from "tubules" or "cristae" analogous to mitochondria. Upon illumination, the cystalline structure develops into a lamellar system as seen in the full-grown chloroplast of Elodea canadensis (Fig. 7a and b). It is indicated that here too the formation of the lamellae proceeds parallel with the pigment synthesis.

Von Wettstein investigated the relation of gene action to the submicroscopic structure of the chloroplasts by employing chlorophyll lethals of barley (von Wettstein, 1957a, 1957b, and 1958). In the development of the barley chloroplast, there is an undifferentiated proplastid with a center core; starch grains accumulate, lamellae are formed, and the plastid as a whole becomes transversed by lamellae. In an albino type, essentially normal early proplastids enclosed by a double membrane were found. There is no differentiation of ordered layers, lamellae, or grana. Differentiation does not proceed beyond the earliest stages, but the plastid increases in size to that of a full-grown chloroplast. The gene change has blocked the development of the submicroscopic chloroplast structure at an early stage, but not the growth of the plastid as a whole. Instead of the

Fig. 7. Electron micrographs of (a, b) developing chloroplast in Elodea canadensis, a higher plant, showing (a) crystalline structure and (b) fully developed chloroplast (Courtesy, Dr. K. Mühlethaler, Eidgenossische Technische Hochschule, Zurich). (c) Chloroplast of Poteriochromonas stipitata, a chrysomonad. 
formation of lamellae, globules accumulate in large masses, and the chloroplast function appears to be arrested. The pigment concentration in the mutant indicates that there is a greater accumulation of chlorophyll $b$, and that the pigments are probably located in the globules. In older stages of the plastids in this mutant, the globules are broken down with further breaks in the inner structure, along with a decrease in pigment concentration.

The picture of chloroplast development still remains inconclusive, and more experimental work is necessary. However, in order for the plant to carry on photosynthesis, an ordered lamellar structure is necessary. As already noted, the chloroplast contains nucleic acid. It is very suggestive that nucleoproteins are involved in the synthesis of the lamellar lipoproteins of the chloroplast, and that the synthesis of chlorophyll and the lamellae proceed simultaneously.

\section{Chloroplast Function as Related to Structure}

What does the chloroplast structure tell us about how the chloroplast functions in photosynthesis? That is, how does the conversion of light energy to chemical energy take place via chlorophyll? The chloroplast is a polyphasic system comprising a stacked array of layers of lipids and proteins separated by monolayers of chlorophyll. Experimentally in Euglena it has been found that any physical or chemical forces (i.e., light, temperature, drugs) that affect the synthesis of either chlorophyll or the chlorophyll-complex will disrupt the lamellar strueture (Wolken et al., 1955; Wolken, 1956a). This change was associated with the removal of $\mathrm{Mg}^{++}$from the chlorophyll molecule. Is the chloroplast, then, like an emulsion, which is stabilized by the chlorophyll molecules? Levitt (1954) suggests that the magnesium atom is the necessary part of the molecule and that any theory of the conversion of light energy into chemical energy via chlorophyll has to take into account the valence changes of the magnesium ion.

Resonance studies indicate that the chloroplast has properties of a crystal and, together w:' 1 the evidence of an ordered structure from electron microscopy, permit an analogy to a photobatterya semiconductor (Calvin, 1958). The assumption is that we are dealing with an electron-transfer phenomenon through many molecules by a conduction-band mechanism. Films of both chlorophyll $a$ and methyl chlorophyllide $a$ deposited from organic solvents have 
been shown to be photoconductive (Nelson, 1957). Monolayers of chlorophyll, chlorophyll plus $\beta$-carotene, or $\beta$-carotene alone, spread on various surfaces, have been demonstrated to be photoconductive (Arnold and Maclay, 1958). The role of the carotenoids in the chloroplast is more controversial. Lynch and French (1957) found that when the chloroplasts were extracted with petroleum ether, the chloroplasts were inactivated, whereas the addition of $\beta$-carotene restored their activity; hence, $\beta$-carotene must be an active component of the photochemical system. This study neglected the role of the lipids in the complex. Fujimori and Livingston (1957), from flash-photolysis studies, also indicated that carotene plays a direct part in the primary act of photosynthesis. On the other hand, Stanier (1958), working with mutants of the purple bacterium Rhodospirillum, and Sager (1958), with mutants of the alga Chlamydomonas, indicate that the carotenoids may act only in catalytic amounts and protect the chlorophyll molecules against high light intensities, temperatures, and oxygen concentrations. Platt (1959) suggests that if the carotenoids are part of the primary sequence, there is a "photosynthetic unit" that can transfer their absorbed energy to a single reaction site.

Chlorophyll and carotenoid in their natural state within the chloroplast do not exist as free pigments but are bound to proteins or lipoproteins. There is an obvious analogy to hemoglobin, where heme is bound to globin, i.e., a chloroglobin. In order to build some models for the chloroplast structure, we have undertaken several experimental approaches.

Chlorophyll-Protein Complex. Investigations of chlorophyll complexed with proteins indicated that some of the complexes have properties similar to those of active chloroplasts (Rodrigo, 1955). We have tried to learn something of the nature of these chlorophyllprotein complexes formed with a variety of proteins. The chlorophyll was extracted from the chloroplasts in 85 per cent acetone (including the carotenoids and other extractables) and was added to various protein solutions $(20 \mathrm{mg} / \mathrm{ml}$ in 0.9 per cent $\mathrm{NaCl}$ ), until no further precipitation occurred. The heavy green chlorophyllprotein precipitate was then washed and resuspended in salt solutions of various concentrations. The stability of the complex was determined from absorption spectra, electrophoresis, and analytical ultracentrifugation. It was found that the extracted chlorophyll complexed more readily with native globulins containing lipid and 
lipoproteins. No complexing could be achieved with the albumins, gelatin, or peptones. In most cases there was difficulty in resolubilizing the precipitate, and pheophytin was formed. The complex was not soluble in reagents which are known to solubilize lipoproteins, but was readily soluble in digitonin. Attempts to demonstrate that these complexes are photoactive were on the whole unsuccessful. The difficulty with this procedure may be that the acetone causes denaturation of the proteins. An analysis of two such complexes, the chlorophyll-horse serum and the chlorophyll- $\gamma$ globulin, is shown in Table 2.

\section{TABLE 2}

Pigment-Protein Complex

\begin{tabular}{cccc}
\hline \multicolumn{1}{c}{ Complex } & $\begin{array}{c}p \\
\left(\begin{array}{c}\text { Moles chloro- } \\
\text { phyll } / 1)\end{array}\right.\end{array}$ & $\begin{array}{c}n \\
(\text { Mg Nitrogen } / \mathrm{ml})\end{array}$ & $\begin{array}{c}\text { M } \\
\text { (Mol wt calcu- } \\
\text { lated from } \\
p \text { and } n \text { ) }\end{array}$ \\
\hline $\begin{array}{c}\text { Chlorophyll } \\
\text { with horse serum } \ldots \ldots \ldots\end{array}$ & $11.4 \times 10^{-5}$ & 1.2 & 69,300 \\
$\begin{array}{c}\text { Chlorophyll } \\
\text { with } \gamma \text {-globulin (bovine) }\end{array}$ & $2.42 \times 10^{-5}$ & 0.62 & 173,000 \\
\hline
\end{tabular}

Chloroplastin. Another possibility was to extract the whole chloroplastic material from the chloroplasts by surface-active agents. Detergents in solution form colloidal aggregates, micelles, consisting of many molecules of detergent clumped together. Such micellar bodies have a very strong attraction for many of the more complex dye molecules. Commercially available non-ionic and ionic surface agents were used to extract the pigment complex from the chloroplasts. The non-ionic recrystallized detergents, Nacconal NRSF $^{3}$ and digitonin, ${ }^{4}$ were found superior to all other surface-active agents tested. These are nitrogen-free detergents and therefore do not interfere with the protein analyses. Although the ionic detergents Alkonal B, glycocholate, and taurocholate were noted as good solubilizing agents, they did not preserve the spectral and other properties of the chlorophyll- complex. The digitonin-extracted pigment-complex therefore serves as the best experimental material.

\footnotetext{
${ }^{3}$ Nacconal NRSF, anionic organic detergent, alkyl aryl sulfonate, Allied Chemical and Dye Corporation.

${ }^{4}$ Digitonin, anionic detergent $\left(\mathrm{C}_{53} \mathrm{H}_{80} \mathrm{O}_{29}\right)$ D-58, Fisher Scientific Co.
} 
This method of obtaining a pigment-complex is similar to that used for the visual complex, rhodopsin, from retinal rods. From the chloroplasts of Euglena and spinach we have extracted in 1.8 per cent recrystallized digitonin a pigment-protein complex containing both chlorophyll and carotenoids (Wolken, 1956b; Eversole and Wolken, 1958). Other such chloroplastins have been extracted previously by Smith (1941a, 1941b, 1941c). We have been able to determine the physical and chemical properties of chloroplastin and to compare them with those of the chloroplast in vivo. The absorption spectrum of chloroplastin closely approximates the absorption spectra of the pigments in the in vivo chloroplasts (Fig. 3 ). Chloroplastin ${ }^{5}$ appears to be a homogeneous complex, judging from its sedimentation in the analytical ultracentrifuge and its electrophoretic pattern (Wolken, 1956b, 1958).

Temperature and Wave Length. The bleaching of chlorophyll in Euglena can be induced by growing the organisms in darkness or, in light, by raising the temperature above $32^{\circ} \mathrm{C}$. Chloroplastin ( $\mathrm{pH} 7.2$ ) can be bleached by light or by heat, analogous to the organisms in vivo. Chloroplastin bleaches at a rate proportional to the amount of light or heat energy absorbed, causing a steady decrease in optical density with the disappearance of the maximum absorption peak at $675 \mathrm{~m} \mu$; it is bleached to pheophytin and unknown products. Below $560 \mathrm{~m} \mu$, bleaching is accomplished by light energy alone; above $560 \mathrm{~m} \mu$, the combined effects of light and heat are required. The total activation energy for bleaching is $48.3 \mathrm{kcal} / \mathrm{mole}$ (Wolken and Mellon, 1957 ).

Photochemistry. Photochemical reactions analogous to photosynthesis were carried out with Euglena chloroplastin, e.g., the rate of photoreduction of the dye, 2,6-dichlorobenzenoneindophenol, the evolution of oxygen, and the conversion of inorganic phosphate to labile phosphate (ATP).

The rate of photoreduction of the dye, 2,6-dichlorobenzenoneindophenol $\left(3 \times 10^{-5} M\right)$, was measured at $600 \mathrm{~m} \mu$ after illumination for 1-4 minutes with 300 foot-candles. In 20 per cent of the preparations, photoreduction of dye was complete within 2 minutes with no accompanying reduction in darkness. The molecular turnover /with a typical preparation indicated that $7 \times 10^{3}$ molecules of

${ }^{5}$ Chloroplastin is not a purified macromolecule as it contains a cytochrome c type haem protein as well as other impurities. 
dye could be photoreduced per molecule of chlorophyll per minute; this is preparation-dependent, and the number may not be quantitatively significant. When the reaction was followed at $488 \mathrm{m \mu}$ (a carotenoid peak), changes in optical density with light or darkness suggested that the carotenoid participates in the reaction (Eversole and Wolken, 1958).

Extracts which actively photoreduced the dye were tested for their ability to cause photolysis or evolution of oxygen. Photolysis was measured manometrically in completely anaerobic Warburg vessels with $\mathrm{KOH}$ in the center well, and the dye was tipped from a side arm at zero time. The reaction system for photolysis measurements was made oxygen-free in order to estimate the oxygen evolved by bacterial bioluminescence. In some preparations of chloroplastin photolysis occurred, yielding 20-30 $\mu$ l oxygen in 2 minutes, with a distinct luminescent glow persisting for almost a minute after a suspension of anaerobic Photobacterium phosphoreum was injected into the experimental vessel in darkness. Controls in the absence of light and those without dye did not evolve oxygen.

In the experiments in which photolysis occurred, a light-catalyzed conversion of inorganic phosphate into labile phosphate (ATP) was measurable. The reaction vessels contained $2 \mathrm{ml}$ of chloroplastin (having a chlorophyll concentration of $10^{-5} \mathrm{M}$ ), $20 \mu$ mole $\mathrm{Mg}^{++}$, $30 \mu$ mole keto-glutarate, $0.3 \mu$ mole riboflavin- 5 -phosphate, $0.6 \mu$ mole menadione, $2 \mu$ mole ascorbate, $5 \mu \mathrm{g}$ cytochrome c, $55 \mu$ mole adenosine monophosphate, and $4 \mu \mathrm{g}$ inorganic phosphate. These experiments were immediately repeated with the addition of glucose and hexokinase, and the glucose-6-phosphate formed was determined by triphosphopyridine nucleotide reduction at $340 \mathrm{~m} \mu$ in the presence of glucose-6-phosphate dehydrogenase. In this way 80-90 per cent of the inorganic phosphate disappearing was accounted for as labile phosphate. The phosphate conversion occurring in the clark control was only $3-4$ per cent of that found in the light.

Electron Microscopy. When a film of chloroplastin was evaporated from a digitonin solution on an electron microscope screen, not fixed, and viewed directly in the electron microscope, there was sufficient electron density to reveal particles of an average diameter of $100 \AA$. A digitonin solution alone when examined in the same manner shows no such electron-dense particles. The electron density of the chloroplastin particles is attributable to the $\mathrm{Mg}^{++}$of the 
chlorophyll molecule and other metals associated with chloroplast. These chloroplastin particles are of the same order of size as those found in the fixed dense lamellae of some chloroplasts; also they could be considered similar to chromatophores. Therefore, it is suggestive that chloroplastin is physiologically active, since the chlorophyll complex remains oriented in the digitonin micellar particles. These particles could contain from 16 to 32 chlorophyll molecules on the surface, as depicted by Frey-Wyssling (1957). The number of molecules that could be packed into this space would be doubled if the chlorophylls were tilted on an angle of $45^{\circ}$, as previously suggested. Such pigment molecules oriented in the digitonin micelle particles could then be the active "photosynthetic units." (See Fig. 6 for electron micrograph of Euglena chloroplast and chloroplastin.)

X-ray Diffraction. Chlorophyll $a$ has been shown to crystallize out in thin sheets $\sim 50 \AA$ thick, perhaps corresponding to bimolecular layers of chlorophyll. The crystallized chlorophyll molecules occupy an area of $\sim 106 \AA^{2}$, and x-ray diffraction patterns obtained from these crystals show strong bands at $7.6 \AA$ and $4 \AA$ (Jacob et al., 1954). X-ray diffraction pictures were obtained by Belavtseva (1957) from crystallized chlorophyll $a$ plus $b$, prepared by petroleum ether extraction in Krasnovskii's laboratory. These patterns showed a strong band at $7.68 \AA$, with average bands at 4.22, 4.06, $3.66,2.78$, and $2.44 \AA$, and weak bands at $2.05,1.83$, and $1.62 \AA$.

$\mathrm{X}$-ray diffraction patterns were prepared at the Mellon Institute for chloroplastin solutions and for chloroplastin films on glass slides. For the solution, strong bands were found at 3.4 to $2.9 \AA$ and another at 2.3 to $2.1 \AA$; for the film, a broad band from 5.3 to $3.9 \AA$ was obtained, with weak lines at $3.35,2.13,2.02,1.82,1.77$, and $1.57 \AA$. These numbers are interplanar spacings. It is difficult at present to interpret these bands; however, the $4 \AA$ band and some of the other lines of chloroplastin in dried films approximate those for the crystallized chlorophyll.

Molecular Weight. Previously suggested molecular weights for chloroplastin have been calculated for leaf extracts (spinach and Aspidistra) prepared in a similar manner with digitonin. These calculations, made from sedimentation rates in the analytical ultracentrifuge, give a molecular weight of the order of 265,000 (Smith, 1941a, 1941b, 1941c). This is a high estimate, due in part to the 
contribution of the digitonin micelle. Digitonin alone forms micelles of minimum molecular weight equal to 75,000. Three such micelles of digitonin are probably linked, yielding a molecular weight of 225,000 (Hubbard, 1954). If the digitonin micellar weight is subtracted from the molecular weight of 265,000 for the complex, the molecular weight of chloroplastin would be of the order of 40,000 (Table 3). Takashima (1952) crystallized from leaf extracts a

\section{TABLE 3}

\section{Analytical Ultracentrifuge Data}

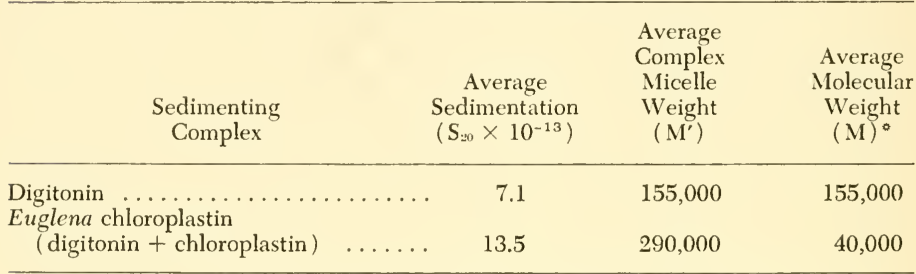

- $\mathrm{M}$ is calculated from $\mathrm{M}^{\prime}$ using the dry weight and per cent nitrogen.

chlorophyll-lipoprotein complex with a molecular weight of 19,000 , determined from diffusion studies. The crystalline complex indicated that there were two molecules of chlorophyll per lipoprotein molecule. A chlorophyll "holochrome" isolated by Smith and Young (1956) from bean seedlings (Phaseolous vulgaris) in glycerine$\mathrm{KOH}$ at pH 9.6 suggests a molecular weight of the order of 400,000 . $^{6}$ For the chloroplasts of the algal flagellates Euglena and Poteriochromonas, the average molecular weights calculated from the geometry of the chloroplast and the pigment concentration were 21,000 and 37,000 , respectively. Using the interference microscope, molecular weights were calculated to be of the order of one-half of these values for the in vivo chloroplast. The molecular weight for chloroplastin, calculated from its sedimentation, nitrogen, pigment concentration, and dry weight, is of the order of 40,000 (Wolken, 1956b). The chloroplastin data imply that there is one chlorophyll molecule to one protein molecule, although the possibility of two or more chlorophyll molecules cannot be excluded (Table 4). FreyWyssling predicts that there are 16 molecules of chlorophyll to one 1959)

${ }^{6}$ The molecular weight of this complex is now reported to be $1 \times 10^{6}$ (Smith, 
macromolecule (on the assumption that the macromolecule is $65 \AA$ in diameter ). Therefore, the chlorophyll-protein complex would have a molecular weight of the order of 68,000 . It must be remembered, though, that chlorophyll could be complexed with different proteins in the various plant species, and that the molecular weights would vary, depending upon the kind of protein. The nature of the protein, or how chlorophyll is complexed to the macromolecule, has not yet been elucidated.

TABLE 4

Analysis of Euglena Chloroplastin

\begin{tabular}{|c|c|c|}
\hline$w$ & dry wt, $\mathrm{mg} / \mathrm{ml}$ & 27.3 \\
\hline$n$ & $\mathrm{mg}$ nitrogen $/ \mathrm{ml} \ldots$ & 0.36 \\
\hline$p$ & moles chlorophyll $1 \ldots \ldots \ldots \ldots \ldots$ & $6.3 \times 10^{-5}$ \\
\hline$p^{\prime}$ & moles chlorophyll $/ 1$ calculated from $w / \mathrm{M}^{\prime}$. . & $9.4 \times 10^{-5}$ \\
\hline$M$ & mol wt calculated from $\mathbf{M}^{\prime} \ldots \ldots \ldots \ldots \ldots \ldots$ & 38,000 \\
\hline M & mol wt calculated from $w, n$, and $p \ldots \ldots \ldots \ldots$ & $30,000-60,000$ \\
\hline
\end{tabular}

\section{Crystallization and Periodicity}

The next steps in our exploration were to see if there were any possible way of accounting for such a lamellar structure for the pigment complex in the chloroplast. Here I would like to refer to some experiments on the Liesegang phenomena (Hedges, 1932). Liesegang observed the formation of periodic structures in the course of staining histological specimens by the Golgi technique (i.e., the impregnation of tissue with potassium dichromate and silver nitrate). The Liesegang phenomenon has been often cited as a model for study of growth and differentiation in living cells. Antigen-antibody reactions in gels also have been compared to Liesegang ring phenomena.

The formation of the rings can be observed if a drop of 15 per cent silver nitrate is placed on a sheet of gelatin which has been impregnated with about 0.4 per cent potassium dichromate. The silver slowly diffuses into the gelatin, there reacts with the potassium dichromate, and silver dichromate is precipitated in the gelatin. The precipitation is not continuous but forms a series of concentric rings separated by clear spaces in the gel. (Fig. 8d.) There are many other such examples; if ferric chloride be added to gelatin and a drop of potassium ferrocyanide solution placed in the center, blue rings of ferriferrocyanide will be formed. Light can modify 
these periodic precipitations if the precipitated molecules are lightsensitive. Many experiments on the Liesegang ring formation have been performed; data on the concentration of reactants, temperature, interspace distances between ring formations, and the time of the reactions have been collected. How much the experimental data can tell us about the chlorophyll-protein specificity or the orientation of chlorophyll in the chloroplast is at present questionable, but it has led us to some interesting considerations on periodic crystallization in colloids and proteins.

Periodic Crystallization. Molecules in solution take up configurations of lowest energy. This leads to crystallization when the number of molecules in the solution exceeds a certain minimum value characteristic of those particular molecules. Such molecular interaction described in terms of crystallization can perhaps be extended to the kinds of molecules that are present in lamellar systems of the chloroplast. An example of such periodic crystallization is that of potassium dichromate in gelatin. The procedure is to place a drop of saturated potassium dichromate in gelatin solution on a microscope slide, warm gently, and quickly transfer the slide to the microscope. Crystallization begins around the periphery of the drop and proceeds in a periodic manner, by alternating periods of rapid and slow growth during which a few relatively large geometrical crystals grow. The distance between the rings decreases with the thickness of the film and with increasing rate of crystallization. (Fig. 8c). The crystallization of sodium chloride is also affected by extremely small concentrations of most colloids (e.g., serum ); as the ions are absorbed on the surface, the sodium chloride molecules are deposited in fine crystals on the glass surface, and periodic rings are formed at certain salt concentrations (Du Noüy, 1926). Digitonin, when it evaporates as a drop on a glass surface, forms periodic rings; however, when a digitonin solution of chloroplastin evaporates as a drop, similar rings develop, but now the chlorophyll concentrates in these rings (Fig. 8a and b). The chlorophyll molecules are carried with the digitonin and become oriented within these rings. When the rings were scanned from the center out with the microspectrophotometer at $675 \mathrm{~m} \mu$, the major absorption peak for chloroplastin, and at $550 \mathrm{~m} \mu$, the minimum absorption peak, it was found that the maximum chlorophyll absorption occurs in the rings, and although the concentration of chlorophyll decreases as it moves out, its concentration remains relatively constant within 

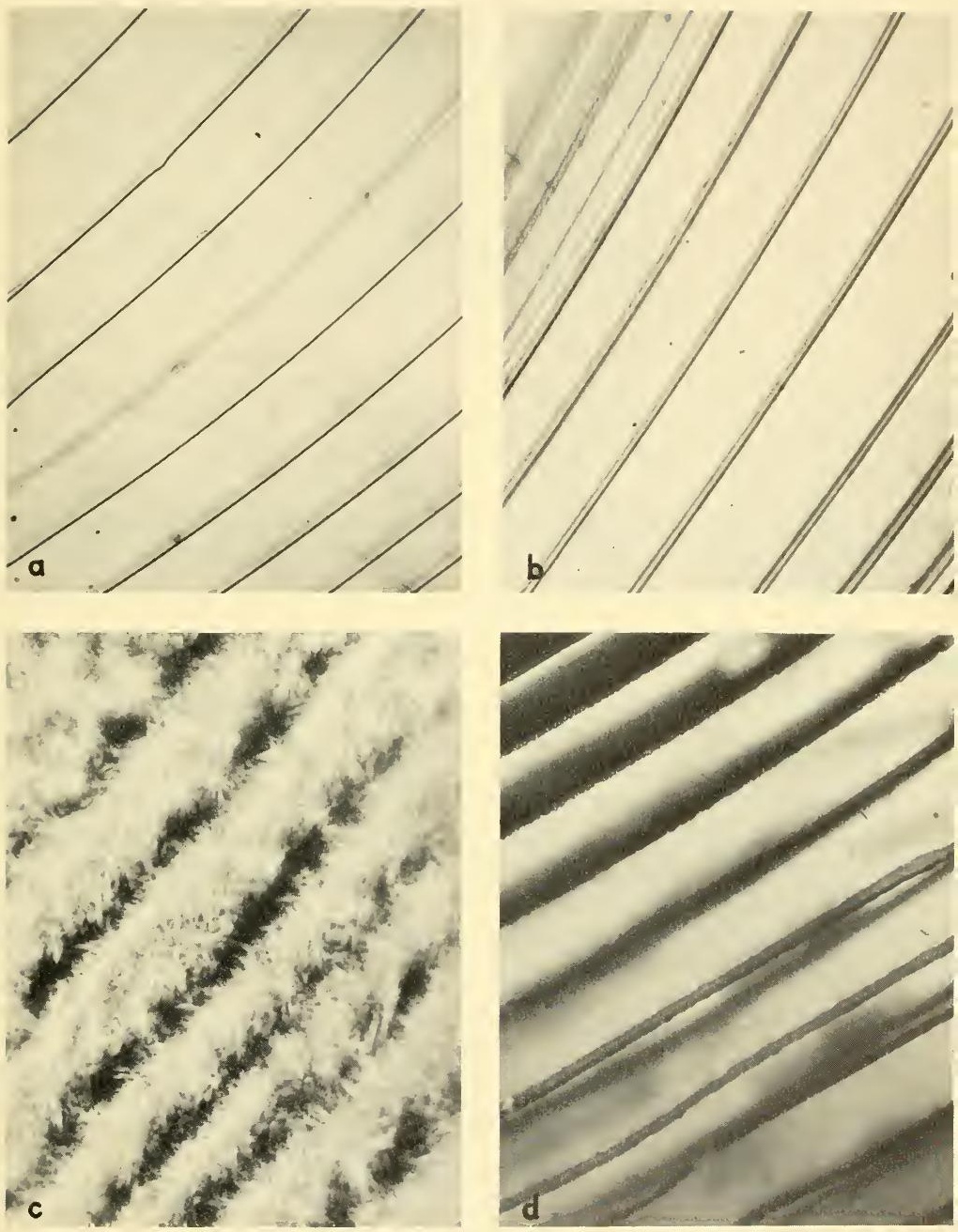

Fig. 8. (a) Periodic ring formation from a 1.8 per cent solution of digitonin evaporated from a drop on a glass surface. $\times 100$. (b) Periodic rings from chloroplastin similarly evaporated from solution. $\times 130$. (c) Periodic crystallization of potassium dichromate from gelatin. $\times 140$. (d) Liesegang ring formation obtained from saturated solution of potassium dichromate in gelatin reacted with silver nitrate. $\times 140$. 


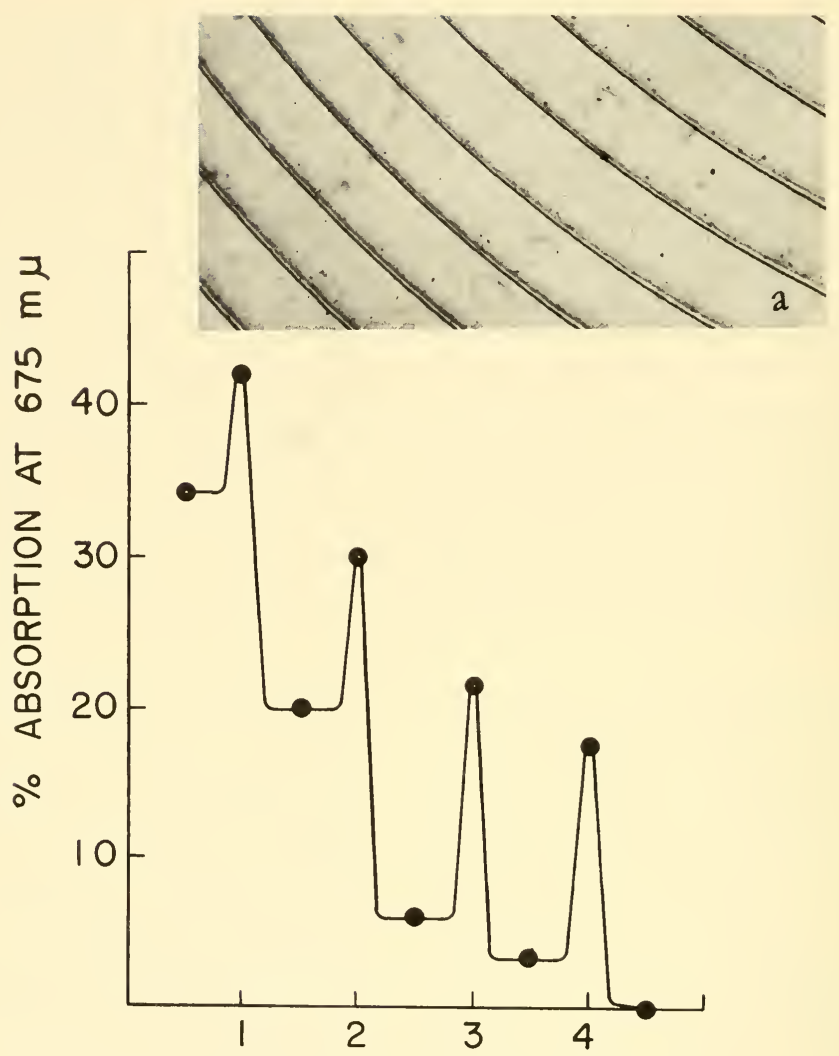

NUMBER OF RINGS

Fig. 9. (a) Periodic rings of chloroplastin prepared as in Fig. $8 \mathrm{~b}(\times 100)$. (b) Chloroplastin rings scanned at $675 \mathrm{~m} \mu$ with the microspectrophotometer, showing that chlorophyll is located within the ring structures.

each ring. (Fig. 9). The chlorophyll complex in digitonin therefore behaves in a fashion similar to salts crystallizing out from colloids, gels, and proteins, and to the Liesegang phenomenon.

Now let us turn to some other experimental studies on the properties of liquid crystals. Liquid crystals generally exist in either the smectic or the nematic paracrystalline state. The more highly 
ordered of these is the smectic, where the molecules are arranged in equidistant parallel layers. Robinson (1958) has shown that polyadenylic acid and polyuridylic acid, when mixed under the right conditions, show a specific interaction, forming crystals. Robinson and Ward (1957) have also shown that when the L and D forms of the synthetic polypeptide, polybenzoylglutamate, are mixed in dioxan, they behave as a liquid crystal. Although the solution was birefringent, no regular orientation was observed with the polarization microscope; but after a short time, orientation appeared on the walls of the capillary and spread toward the center with a very regular orientation.

If digitonin is caused to flow through a capillary, it becomes birefringent when observed through crossed polaroids; a similar phenomenon is observed with chloroplastin. This is due to the orientation of the particles in the flowing stream. The chlorophylls then become oriented within the digitonin micelles. If chloroplastin is allowed to stand, no birefringence is observed. However, a liquid crystalline phase will separate out; under certain conditions, birefringent rodlike fibers (tactoids) form. These observations are similar to those made on tobacco mosaic virus (Lauffer et al., 1949).

Quantitative theories have been proposed to explain liquid crystals as being the result of anisotropic interactions between long, rodlike molecules. These theories have the essential feature that long rods can be packed economically in a given space only if they are aligned. Hence, a concentrated solution of rodlike molecules tends to form liquid crystal structures, but in dilute solutions, isotropic structures occur (Zimm, 1959). For a more complete discussion of liquid crystals, reference should be made to the Discussions of the Faraday Society (1958) and Transactions of the Faraday Society (1933).

These studies suggest that chloroplastin possesses properties of a liquid crystal and because of these properties, structural as well as photochemical integrity is maintained. Its behavior then is similar to that of the in vivo chloroplast. The lamellar structure may be an efficiency mechanism rather than a critical functioning device. However, this does not exclude the probability that a liquid crystal type of matrix is necessary for orientation of the active pigment complex. 


\section{References}

Annold, W., and H. K. Maclay. 1958. In The Photochemical Apparatus: Its Structure and Function. Brookhaven Symposia in Biol. No. 11: 1-9.

Bass-Becking, L. G. M., and E. A. Hanson. 1937. Note on the mechanism of photosynthesis. Proc. K. Nederland. Akad. Wetensch 40: 752-755.

Belavtseva, E. M. 1957. Electron diffraction and electron microscope studies of chlorophyll $a$ and $b$ and Gramicidin. Biophysics (U.S.S.R.) (English Translation) 2: 617-622.

Boulder Conference on Biophysics. 1959. Revs. Modern Phys. 31: Nos. 1, 2.

Brookhaven Symposia in Biology, No. 11. 1958. The Photochemical Apparatus: Its Structure and Function.

Calvin, M. 1958. In The Photochemical Apparatus: Its Structure and Function. Brookhaven Symposia in Biol. No. 11: 160-180.

Calvin, M. 1959a. Energy reception and transfer in photosynthesis. Revs. Modern Phys. 31: 147-156.

Calvin, M. 1959b. Free radicals in photosynthetic systems. Revs. Modern Phys. 31: 157-161.

Discussions of the Faraday Society. 1958. Configurations and Interactions of Macromolecules and Liquid Crystals. No. 25.

Du Noüy, P. Lecompte. 1926. Surface Equilibria of Biological and Organic Colloids. The Chemical Catalog Co., New York.

Elbers, P. F., K. Minnaert, and J. B. Thomas. 1957. Submicroscopic structure of some chloroplasts. Acta Botan. Neerl. 6: 345-350.

Eversole, R. A., and J. J. Wolken. 1958. Photochemical activity of digitonin extracts of chloroplasts. Science 127: 1287-1288.

Frey-IVyssling, A. 1957. Macromolecules in Cell Structure. Harvard University Press, Cambridge, Mass.

Fujimiori, E., and R. Livingston. 1957. Interactions of chlorophyll in its triplet state with oxygen, carotene, etc. Nature 180: 1036-1038.

Gaffron, H. (ed.). 1957. Research in Photosynthesis. Interscience Publishers, Inc., New York.

Goedheer, J. C. 1955. Orientation of the pigment molecules in the chloroplast. Biochim. et Biopluys. Acta 16: 471-476.

Goedheer, J. C. 1957. Optical properties and in vivo orientation of photosynthetic pigments. Thesis. State University of Utrccht, Netherlands.

Granick, S. 1955. In W. Ruhland (ed.). Handbuch der Pflanzenphysiologie. Springer Verlag, Berlin. 1: 507-564.

Hedges, E. J. 1932. Liesegang Rings and Other Periodic Structures. Chapman and Hall, Ltd., London.

Heitz, E. 1955. Über die Struktur der Chromosomen und Chloroplasten. Nova Acta Leopoldina 17: 517-540.

Hodge, A. J. 1959. Fine structure of lamellar systems as illustrated by chloroplasts. Revs. Modern Phys. 31: 331-341.

Hodge, A. J., J. D. McLean, and F. V. Mercer. 1955. Ultrastructure of the lamellae and grana in the chloroplasts Zea Mays L. J. Biophys. Biochem. Cytol. 1: 606-614.

Hubbard, R. 1954. The molecular weight of rhodopsin and the nature of the rhodopsin-digitonin complex. J. Gen. Physiol. 37: 381-399.

Јасов, E. E., A. E. VAtTer, and A. S. Holt. 1954. Crystalline chlorophyll and bacteriochlorophyll. Arch. Biochem. Biophys. 53: 228-238.

Lauffer, M. A., W. C. Price, and A. W. Petre. 1949. The nature of viruses. Advances in Enzymol. 9: 171-240.

Levitt, L. S. 1954. The role of magnesium in photosynthesis. Science 120: 33-35.

LEYON, H. 1956. The structure of the chloroplasts. Svensk Kem. Tidskr. 68: 70-89.

Lynch, V. H., and C. S. French. 1957. B-carotene, an active component of chloroplasts. Arch. Biochem. Biopliys. 70: 382-391. 
Mühlethaler, K. 1955. The structure of chloroplasts. Intern. Rev. Cytol. 4: 197220.

Mühlethaler, K. 1959. Private communication.

Nelson, R. C. 1957. Some photoelectric properties of chlorophyll. J. Chem. Phys. 27: 864-867.

Platt, J. R. 1959. Carotene-donor-acceptor complexes in photosynthesis. Science 129: $372-374$.

Rabinowitch, E. I. 1945. Photosynthesis and Related Processes. Interscience Publishers, Inc., New York. Vol. 1. Pp. 355-480.

Rabinowitci, E. I. 1951. Photosynthesis and Related Processes. Interscience Publishers, Inc., New York. Vol. 2, part 1. Pp. 603-736.

Rabinowitch, E. I. 1956. Photosynthesis and Related Processes. Interscience Publishers, Inc., New York. Vol. 2, part 2. Pp. 1714-1758.

Robinson, C. 1958. In J. R. Danielli, K. G. A. Pankhurst, and A. C. Riddiford (eds.). Surface Phenomena in Chemistry and Biology. Pergamon Press, Ltd., London.

Robinson, C., and S. C. WARD. 1957. Liquid-crystalline structures in polypeptides. Nature 180: 1183-1184.

Rodrigo, F. A. 1955. Experiments concerning the state of chlorophyll in the plant. Thesis. State University of Utrecht, Netherlands.

SAger, R. 1958. In The Photochemical Apparatus: Its Structure and Function. Brookhaven Symposia in Biol. No. 11: 101-117.

Sager, R., and G. E. Palade. 1957. Structure and development of the chloroplast in Chlamydomonas. I. The normal green cell. J. Biophlys. Biochcm. Cytol. 3: 463-488.

Sissakian, N. M. 1958. Enzymology of the plastids. Advances in Enzymol. 20: 201236.

Sмiтн, E. L. 1941a. The chlorophyll-protein compound of the green leaf. J. Gen. Physiol. 24: 565-582.

Smith, E. L. 1941b. The action of sodium dodecyl sulphate on the chlorophyll-protein compound of the spinach leaf. J. Gen. Physiol. 24: 583-596.

Sмiтн, E. L. 1941c. The effects of detergents on the chlorophyll-protein compound of spinach as studied in the ultracentrifuge. J. Gen. Physiol. 24: 753-764.

Sмгтн, J. H. C. 1959. Proc. Intern. Botan. Congr. 9th Congr., Montreal. In press.

Sмith, J. H. C., and V. M. K. Young. 1956. Chlorophyll formation and accumulation in plants. In A. Hollaender (ed.). Radiation Biology. McGraw-Hill Book Co., Inc., New York. Vol. 3. Pp. 393-442.

Stanier, R. Y. 1958. In The Photochemical Apparatus: Its Structure and Function. Brookhaven Symposia in Biol. No. 11: 43-53.

Strother, G. K., and J. J. Wolken. 1959. A simplified microspectrophotometer using photoconductive cells. Science 130: 1084-1088.

TaKashima, S. 1952. Chlorophyll-lipoprotein obtained in crystals. Nature 169: 182183.

Thomas, J. B. 1955. Structure and functions of the chloroplast. Prog. in Biophys. and Biophys. Chem. 5: 109-139.

Thomas, J. B. 1958. Chloroplast structure and function. Endeavour 17: 156-161.

Transactions of the Faraday Society. 1933. Theory of Liquid Crystals. 29, part 2: 883-1085.

Trunnit, H. J., and G. Colmano. 1958. Chloroplast studies. I. Absorption of chlorophyll monolayers at liquid interfaces. Biochim. et Biophys. Acta 30: 435447.

von Wettstein, D. 1957a. Chlorophyll-Letale und der submikrosopische Formwechsel der Plastiden. Exptl. Cell Research 12: 427-506.

von Wettstein, D. $1957 \mathrm{~b}$. Genetics and the submicroscopic cytology of plastids. Heditas 43: 303-317.

von Wettstein, D. 1958. In The Photochemical Apparatus: Its Structure and Function. Brookhaven Symposia in Biol. No. 11: 138-159. 
Wolken, J. J. 1956a. A molecular morphology of Euglena gracilis var. bacillaris. J. Protozool. 3: 211-221.

Wolken, J. J. 1956b. Photoreceptor structures. I. Pigment monolayers and molecular weight. J. Cellular Comp. Physiol. 48: 349-370.

Wolken, J. J. 1958. In The Photochemical Apparatus: Its Structure and Function. Brookhaven Symposia in Biol. No. 11: 87-100.

Wolken, J. J. 1959. The structure of the chloroplast. Ann. Rev. Plant Physiol. 10: 71-86.

Wolken, J. J., and A. D. Mellon. 1956. The relationship between chlorophyll and the carotenoids in the algal flagellate, Euglena. J. Gen. Physiol. 39: 675-685.

Wolken, J. J., and A. D. Mellon. 1957. Light and heat in the bleaching of chloroplastin, Euglena. Biochim. et Biophys. Acta 25: 267-274.

Wolken, J. J., A. D. Mellon, and C. L. Greenblatt. 1955. Environmental factors affecting growth and chlorophyll synthesis in Euglena. 1. Physical and chemical. 2. The effectiveness of the spectrum for chlorophyll synthesis. J. Protozool. 2: 89-96.

Wolken, J. J., and G. E. Palade. 1953. An electron microscope study of two flagellates. Chloroplast structure and variation. Ann. N. Y. Acad. Sci. 56: 873881.

Wolken, J. J., and F. A. Schwertz. 1953. Chlorophyll monolayers in chloroplast. J. Gen. Physiol. 37: 111-120.

Zimi, B. H. 1959. Concentrated macromolecular solutions. Revs. Modern Phys. 31: 123-129.

\section{DISCUSSION}

\section{A. Hodge, J. J. Wolken, A. C. Giese}

Dr. Hodge (Massachusetts Institute of Technology): Would you please indicate the conditions under which the chloroplastin rings form?

Dr. Wolken: The simplest procedure is to place a drop of chloroplastin on a clean microscope slide, warm gently, and then quickly transfer the slide to the microscope.

Dr. Giese (Stanford University): Is there any suggestion of the derivation of chloroplasts from cilia, as there is in the case of rods and cones?

Dr. Wolken: I do not think that the chloroplasts are derived from the cilia. However, it is quite likely that the chloroplast lamellae are formed from fibrillar protein macromolecules. 


\section{Lamellar Systems in Myelin and Photoreceptors}

as Revealed by High-Resolution

Electron Microscopy

H. FERNÁNDEZ-MorÁN ${ }^{2}$

\section{Introduction}

Ultrastructure studies (Engström and Finean, 1958; FernándezMorán, 1959b; Fernández-Morán and Brown, 1958; Schmidt, 19.37; Schmitt, 1944; Schmitt et al., 1935) of the regularly arranged submicroscopic layers which build up the nerve myelin sheath, photoreceptors, chloroplasts, and numerous other types of "lamellar systems," have furnished the basis for a broad approach to related problems of fundamental interest in the field of molecular biology. Current biophysical and biochemical investigations are therefore concerned not only with the nature of these multilayered structures considered as specialized derivatives of the cell membrane, but also with the distinctive repetitive features of their periodic array which seem to underlie the specific processes of energy reception and transfer in living organisms.

Many lamellar structures may be regarded as model systems which are particularly favorable for detailed investigation of cell-

1 This work was supported by Atomic Energy Commission Contract AT(30-1)2278 and by a grant ( C-3174) from the National Institutes of Health. The author is particularly indebted to Dr. William H. Sweet, Associate Professor of Neurosurgery, Harvard Medical School, and to Dr. Raymond D. Adams, Bullard Professor of Neuropathology, Harvard Medical School, for their generous assistance and support of this project. It is also a pleasure to thank Professor Samuel C. Collins, Director of the Cryogenic Engineering Laboratory, M. I. T., for his guidance and kind help in extending to us the facilities of his laboratory during the course of the liquid helium experiments; and his assistant, Robert Cavileer, for his technical contribution in the design and instrumentation of the liquid helium experiments. Sincere thanks are also due to Frederick B. Merk, Ernest Shmid, and Joanne T. Frederick for their able technical assistance; and to Gertrude Dole and Irene Brierley for their valuable help in preparing the manuscript.

2 Mixter Laboratories for Electron Microscopy Neurosurgical Service, Massachusetts General Hospital; and Department of Neurology and Psychology, Harvard Medical School, Boston, Massachusetts. 
membrane organization in the living state because they are composed almost exclusively of numerous unit membranes multiply wrapped or folded in highly ordered complexes. These compact aggregates of oriented membranes are well suited for analysis by polarized light and x-ray diffraction, which can often be performed on the intact components under physiological conditions (Schmitt, 1944; Schmitt et al., 1935).

In the embryogenesis of peripheral nerve myelin, Geren and Schmitt (1955) have shown that the Schwann cells "wrap themselves" many times around an outgrowing axon by a continuous infolding of the outer Schwann-cell surface membrane (Schmitt, 1959, p. 460). After numerous double membranes are thus spirally wrapped about the axon, the layers become condensed to form the compact myelin sheath (Geren and Schmitt, 195.5; Robertson, 1959), which exhibits strong birefringence (Schmidt, 1937), indicating a high degree of orientation of the constituent lipid-protein units, and other physical properties characteristic of the smectic fluid-crystalline state (Schmidt, 1937; Schmitt, 1959).

The analysis of the myelin sheath ultrastructure is a classic demonstration of the advantages inherent in an approach combining direct and indirect methods (Schmidt, 1937; Fernández-Morán, $1959 \mathrm{~b}$ ). Based on the polarized-light investigations (Schmidt, 1937; Schmitt, 1944) begun over a century ago, and on subsequent lowangle x-ray diffraction studies (Schmitt et al., 1935), the concentric laminated structure of the sheath was accurately predicted, and the thickness of the layers deduced, before the electron microscope provided direct confirmation of the postulated multilayer arrangement (Fernández-Morán, 1950; Robertson, 1959).

Electron microscopy is the only method that permits direct visualization of structural details of molecular dimensions within a selected region and thus furnishes data well above the level of statistical uncertainty commonly associated with indirect analytical methods. However, electron microscope investigations are severely limited by numerous preparation artifacts resulting from complex perturbations of the living state when biological systems are subjected to fixation, dehydration, embedding, and ultrathin-sectioning procedures, and finally to the deleterious effects of high-vacuum and electron-beam irradiation during observation.

Practically all of our present direct knowledge of lamellar systems, and of cellular fine structure in general, is based on electron 
microscope examination of osmium-fixed specimens (FernándezMorán and Brown, 1958; Porter, 1957). Although this type of preparation has already yielded much valuable information, it is commonly acknowledged that we are merely disclosing the refractory macromolecular framework of a far more intricate dynamic system. Thus, the concentric array of dense bands and light interspaces revealed by electron microscopy in the nerve myelin sheath represents merely the "osmium-stabilized skeleton" (Fernández-Morán, 1957), or the pattern of selective deposition of osmium at certain sites, without permitting identification of specific regions containing lipids, lipoproteins, or protein constituents (Fernández-Morán and Finean, 1957). Within this altered matrix, deprived of the fundamental water component, there is scant possibility of localizing or even detecting the numerous enzymes, electrolytes, trace metals, and other important constituents associated with the myelin sheath (Fernández-Morán, 1959b; Lumsden, 1957).

The shortcomings of our present preparation techniques are more acutely felt now that modern electron microscopes consistently achieve resolutions of the order of 7 to $10 \AA$, and are thus inherently capable of directly visualizing molecular structures in the size range of certain enzymes and hydrated lipid-protein complexes, particularly when these are incorporated in the periodic multilayer arrangement characteristic of lamellar systems. The development of adequate preparation methods is therefore a major problem which must be solved before high-resolution electron microscopy can be more effectively applied in the study of biological systems during growth and function.

Low-temperature preparation techniques provide one of the most promising approaches, since rapid freezing of biological specimens suspends all physiological activity, immobilizing and preserving tissue constituents (Harris, 1954). That cooling to temperatures close to absolute zero does not appreciably impair critical life processes has now been amply demonstrated by the survival of a wide variety of living organisms, including bacteria, spermatozoa, and many other sensitive cells and tissues, which are first treated protectively with glycerol, then frozen with liquid nitrogen or liquid helium, and subsequently thawed in a controlled manner (Harris, 1954; Lovelock, 1953; Parkes, 1951). By ultrarapid cooling to low enough temperatures, it may be feasible to preserve the original position and relationship of the main organic and inorganic con- 
stituents of cellular organization in tissues, including the predominant water component, the transient intermediates with unpaired electron-spin (which participate in enzymatic reactions and metabolic electron transfer), and other unstable chemical species generally referred to as free radicals (Broida, 1957; Minkoff, 1959). However, in order to achieve this optimum preservation and effectively "fix" the highly reactive free radicals in biological systems, it would be necessary to work at temperatures of liquid helium (Allen, 1952; Mendelssohn, 1956), which are about $100^{\circ} \mathrm{C}$ lower than those currently obtained with isopentane-liquid nitrogen coolants.

Improved low-temperature preparation techniques for electron microscopy of biological tissues (Fernández-Morán, 1959a) have recently been developed which vield better morphological and histochemical preservation of lamellar systems and other cell components than do the standard freeze-drying or freeze-substitution methods. These "cryofixation techniques" (Fernández-Morán, $1959 \mathrm{c}$ ) are based on rapid freezing of fresh or glycerinated tissues with liquid helium II at $1^{\circ}$ to $2^{\circ} \mathrm{K}$, followed by freeze-substitution and embedding in plastics at low temperatures, under conditions which minimize ice-crystal formation, artificial osmotic gradients, and extraction artifacts.

The following review deals with salient features of the fine structure of lamellar systems in thin sections of the myelin sheath and selected photoreceptors, as revealed by high-resolution electron microscopy, using both standard preparation techniques and the new low-temperature procedures. Although the latter are still in a preliminary stage of development, the experimental approaches will be outlined and the underlying operational concepts discussed in order to illustrate the potentialities of ultrastructure research at low temperatures (Fernández-Morán, 1959d). Lamellar systems are particularly suitable for this type of work, since all steps of the preparation procedures can be followed and the artifact sources analyzed directly at low temperatures by combined application of $\mathrm{x}$-ray diffraction techniques and electron microscopy. Moreover, by cooling the object during observation in the microscope, and using an electron microbeam of very low intensity, irradiation damage and specimen contamination can be minimized (FernándezMorán, 1959a, 1959d). With this experimental arrangement for low-temperature electron microscopy, a direct investigation of ice- 
crystal structure and growth has already been undertaken (Fernández-Morán, 1959d). Further developments along these lines may eventually permit direct electron-optical studies of thin biological specimens in which the native hydrated state has been partially preserved through vitrification at low temperatures.

\section{Low-Temperature Preparation Techniques for Electron Microscopy}

Standard Freeze-Drying and Freeze-Substitution Techniques. The classical freeze-drying techniques (Gersh and Stephenson, 1954) of established value in morphological and histochemical studies of tissues by light microscopy have generally proved to be inadequate for electron microscope preparations. Even when freezedrying is performed under favorable conditions (Sjöstrand and Baker, 1958), the resulting thin sections show extensive vacuolization due to ice-crystal formation, and generally deficient preservation of tissue structure. This ice-crystal artifact plays a major role in all low-temperature procedures, and can be largely accounted for in terms of the characteristic phase transformations which water undergoes at different temperatures. If a sufficiently small tissue sample is cooled rapidly enough (in less than $2 / 1000$ second) below $-100^{\circ} \mathrm{C}$ with isopentane-liquid nitrogen (Stephenson, 1956), the water in the tissue will solidify in a metastable "vitreous" or glassy state (Gersh and Stephenson, 1954). In the case of pure water, this vitreous ice turns abruptly into crystalline ice above the critical "glassy transformation temperature," around $-130^{\circ} \mathrm{C}$ (Meryman, 1956). The phase transformations of water in tissues are different from those in pure water (Luyet, 1957; Stephenson, 1956), but even in tissues a transition temperature of about $-100^{\circ} \mathrm{C}$ has been assumed by Stephenson (1956). Meryman (1956) has shown that a pure ice crystal can develop in 30 seconds from the glassy state to a length of $1 \mu$ at $-70^{\circ} \mathrm{C}$. Since the sublimation of ice in freezedrying is usually carried out at temperatures not below $-80^{\circ} \mathrm{C}$, the rapid growth of ice crystals within the tissue matrix and the ensuing damage occurring during the relatively long periods of vacuum dehydration are readily understandable.

In the freeze-substitution process introduced by Simpson (1941), the ice formed within the tissue is slowly dissolved or "substituted" in a fluid solvent at temperatures of about $-70^{\circ} \mathrm{C}$ (Patten and 
Brown, 1958). Although addition of chemical fixatives to the substituting fluids (Feder and Sidman, 1958; Fernández-Morán, 1957) improves the quality of tissue preservation, the artifacts associated with the formation of ice crystals during prolonged immersion at these temperatures-well above the critical transition at $-100^{\circ} \mathrm{C}-$ cannot be avoided. In both freeze-drying and freeze-substitution, embedding of the tissues produces further extraction and rearrangements which, though indiscernible under the light microscope, nevertheless obliterate important structural detail at the submicroscopic level. The partially undenatured, freeze-substituted tissue components are particularly vulnerable to the lipid-extracting action of the usual transfer and embedding media. All of these artifact sources severely limit the usefulness of standard freeze-substitution techniques for electron microscopy.

In order to adapt freeze-substitution for the study of tissue finestructure, it would be necessary to dissolve the ice matrix at temperatures preferably below the critical glassy transition in tissues, tentatively assumed to be at $-100^{\circ} \mathrm{C}$. The entire process of impregnation and embedding would also have to be carried out at temperatures of $-100^{\circ} \mathrm{C}$ to approximately $-50^{\circ} \mathrm{C}$, at which the solubility of the labile lipoprotein membrane complexes would be greatly reduced, and the stiffness of the frozen tissue matrix would prevent gross structural rearrangements from occurring. Muller (1957), who first successfully applied photopolymerization of methacrylate at $-10^{\circ} \mathrm{C}$ for embedding of frozen-dried preparations, noted marked improvements in the preservation of chloroplast finestructure, and considerably reduced extraction of lipoprotein complexes. Freeze-substitution procedures are well suited for infiltration with a monomer and photopolymerization at low temperatures, since, as Feder and Sidman (1958) have pointed out, the transitions do not involve the disruptive effects of a liquid-gas interface sweeping through the specimen, as is the case when frozen-dried tissues are immersed in the embedding fluids. As shown by previous studies (Fernández-Morán, 1950, 1957), treatment of the tissues with glycerol or other protective agents prior to freezing is essential to prevent destructive ice-crystal formation in the more commonly encountered larger tissue specimens.

Cryofixation and Related Low-Temperature Techniques. Guided by these considerations, and based on the results of earlier work (Fernández-Morán, 1950, 1957) and the recent technical 
contributions by Feder and Sidman (1958) and by Miiller (1957), improved low-temperature preparation methods have been developed for the study of tissues by high-resolution electron microscopy (Fernández-Morán, 1959a, 1959c, 1960). The designation "cryofixation techniques” (Femández-Morán, 1959c) was suggested to distinguish these procedures from the related freeze-substitution methods (Feder and Sidman, 1958; Patten and Brown, 1958; Simpson, 1941) because of the different type of approach, involving freezing with liquid helium II and low-temperature polymerization, which will become of increasing operational value in the course of further developments.

The basic cryofixation techniques comprise sequential application of the following procedures: (1) rapid or ultrarapid freezing of thin, fresh specimens or of glycerinated tissues with liquid helium II at $1^{\circ}$ to $2^{\circ} \mathrm{K}$; (2) staining at $-150^{\circ} \mathrm{C}$ with halogens or organometallic compounds dissolved in isopentane; (3) substitution of the ice matrix with solutions of heavy-metal salts in alcohol-acetone and alkyl halide mixtures, or alternatively in certain organometallic compounds, at temperatures of $-130^{\circ}$ to $-80^{\circ} \mathrm{C}$; (4) infiltration of the specimens at $-100^{\circ}$ to $-80^{\circ} \mathrm{C}$ with acrylic monomers; and (5) final embedding of the specimens by photopolymerization with ultraviolet light at temperatures of $-80^{\circ}$ to $-20^{\circ} \mathrm{C}$ (Fox et al., 1958).

In an important technical variant used mainly for examination of specimens which have been subjected to a minimum of chemical fixation, all intermediate staining procedures are eliminated, and only freeze-substitution is carried out with acetone-ethyl chloride mixtures at temperatures of about $-130^{\circ}$ to $-110^{\circ} \mathrm{C}$, which are still below the critical transition range for tissues.

A detailed account of the cryofixation and related low-temperature preparation procedures is now being prepared for publication ${ }^{3}$ and only representative methodological aspects will be reviewed here.

Freezing of Biological Specimens With Liquid Helium II. The use of liquid helium as an indispensable refrigerant for attaining temperatures close to absolute zero is based on important recent developinents in the fields of low-temperature chemistry (Klein and Scheer, 1958) and free-radical research (Broida, 1957; Minkoff, 1959). The temperature domain below the glassy transformation of

${ }^{3}$ H. Fernández-Morán. Ultrastructure of the Nervous System. Part I, Techniques. ( To be published by Academic Press, Inc., New York.) 
water at $-130^{\circ} \mathrm{C}$, which had been set aside by the biologist as a "sanctuary" protecting all life processes from further change, corresponds in fact to the upper limit of the vigorous new field of lowtemperature chemistry. This discipline concerns itself mainly with the wide variety of low-temperature reactions which occur below $150^{\circ} \mathrm{K}$ and are characterized by low activation energies of $5 \mathrm{kcal} /$ mol or less. The studies of Klein and Scheer (1958) have demonstrated that numerous chemical reactions involving the addition of hydrogen atoms to solid olefins readily occur at $-195^{\circ} \mathrm{C}$ with measurable activation energies, and also evidence of diffusion processes which are of considerable importance at low temperatures. Recently, these authors have shown that even at $20^{\circ} \mathrm{K}$ hydrogen atoms will react with solid oxygen. Free radicals and many other unstable chemical species will exhibit considerable reactivity at $70^{\circ} \mathrm{K}$ (Broida, 1957), and the trapping of these transient intermediates by freezing into an inert solid at liquid helium temperatures (Minkoff, 1959) has been the subject of considerable investigation. Free radicals and other intermediates with umpaired electron-spin play an important role in many enzymatic reactions and in photosynthetic processes (Calvin, 1959a). The possibility of stabilizing these highly reactive intermediates in intact biological systems, which can be adequately achieved only at liquid helium temperatures, would therefore amply justify its application.

Normal liquid helium I shows poor heat conductivity and is therefore less suitable than its low-temperature phase, known as liquid helium II, for direct, rapid cooling of biological specimens. Helium II, which is obtained by evaporating ordinary liquid helium under reduced pressure until the temperature falls below the critical $\lambda$-point $\left(2.19^{\circ} \mathrm{K}\right)$, exhibits the unique properties of heat superconductivity and superfluidity (Allen, 1952; Mendelssohn, 1956). Under certain conditions, the bulk liquid helium II will conduct heat 10,000 times better than will copper, and it can flow rapidly through the finest capillaries without any viscous drag. These phenomena are restricted to a narrow temperature range, and transfer of heat from a specimen into the bulk liquid is actually impeded by a poorly conducting boundary layer (Allen, 1952; Mendelssohn, 1956). However, despite the existence of this thermal boundary resistance, helium II appears to be the most effective refrigerant for direct cooling of thin biological specimens to temperatures which are $100^{\circ} \mathrm{C}$ lower than those obtained with the standard isopentane- 
liquid nitrogen mixtures (Fernández-Morán, 1959c). Attainment of these extremely low temperatures, at which all other substances solidify, and at which diffusion or recombination of unstable chemical species within the tissue matrix can be practically eliminated, is therefore the main reason for the use of liquid helium II in connection with ultrastructure studies. Although better preservation of fine structure has already been achieved at this preliminary stage, it must be emphasized that the full benefits of this approach are to be expected only if the trapped free radicals can be effectively immobilized and made electron-optically visible within the intact tissues by suitable interaction with heavy atoms and (addition) polymerization at lower temperatures. Liquid helium is chemically inert and far safer to handle than isopentane-propane-liquid nitrogen mixtures or liquid hydrogen. Moreover, the widespread use of liquid helium in industrial cryogenics research now makes it more readily available, and the practical difficulties connected with its application can be easily solved once the basic experimental techniques have been mastered.

In collaboration with Professor Samuel C. Collins and his associates at the Cryogenic Engineering Laboratory of the Massachusetts Institute of Technology, a series of preliminary experiments with liquid helium II have been carried out during the past year. As shown in Fig. 1, the basic equipment consists of a specimen stage attached to a shielded helium Dewar which can be evacuated with a large forepump of $311 \mathrm{cu}$. ft./min. capacity. For processing even a limited number of specimens it is necessary to prepare relatively large amounts of bulk liquid helium II because of its small heat of vaporization. This is accomplished by filling the inner Dewar with liquid helium I and rapidly lowering the vapor pressure to approximately $70 \mu$, equivalent to $-272.2^{\circ} \mathrm{C}$. The simple type of stage consisting essentially of a large glass stopcock makes it possible to introduce fresh or glycerinated tissues into the liquid helium II bath without breaking the vacuum (Fernández-Morán, 1959d). Highspeed photographs, recorded in collaboration with D. Eldridge of the Electrical Engineering Department of M. I. T., show that immersion of the specimen into the bulk liquid does not produce boiling (Figs. 2, 3), suggesting that equilibration of the temperature difference takes place very rapidly. If necessary, the specimen can be precooled with propane-liquid nitrogen or liquid hydrogen and immediately plunged into liquid helium II to prevent transitional 


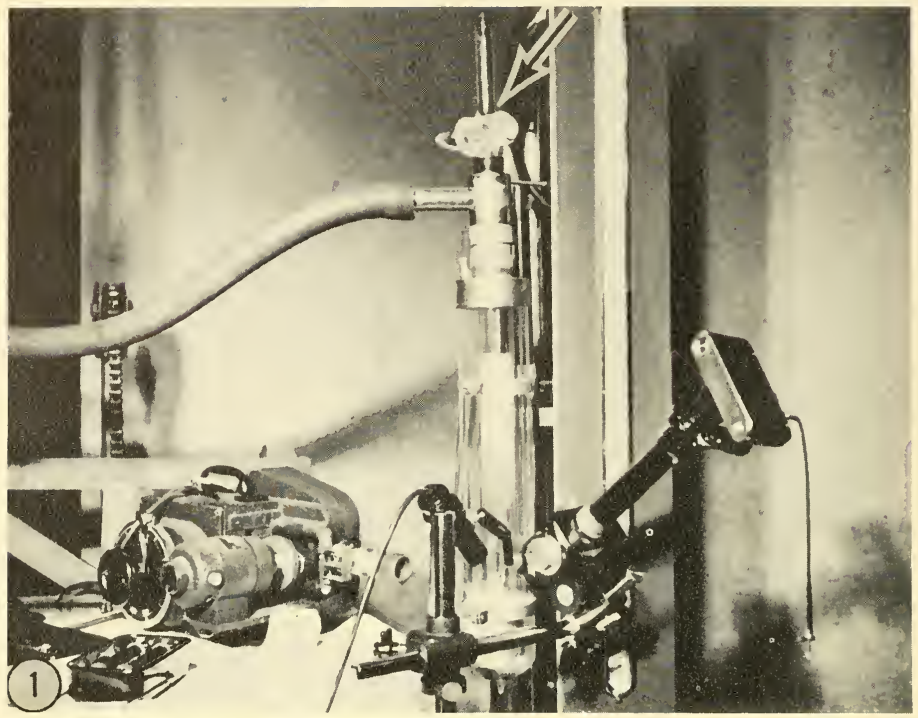

Fig. 1. Equipment for rapid freezing of biological specimens in liquid helium II with attached gas thermometer, high-speed camera, and microscope for direct observation of specimens within helium Dewar at $-272^{\circ} \mathrm{C}$. Arrow indicates specimen stage for introducing fresh or glycerinated tissues into liquid helium II without breaking vacuum. (Installed at the Cryogenic Engineering Laboratory, M. I. T., in cooperation with Professor Samuel C. Collins.)

changes from occurring. However, very rapid cooling leads to severe distortion and cracking of larger tissue sections, and the optimum cooling velocity must therefore be determined for each type of specimen.

A simplified version of this equipment has proved to be very useful for achieving rapid and ultrarapid cooling of tissues with other types of refrigerants. Thus, if liquid nitrogen is cooled close to its fusion temperature $\left(63.15^{\circ} \mathrm{K}\right)$ by evacuating the Dewar, small tissue specimens can be introduced through the attached vacuum stage, and appear to freeze more rapidly in this quiescent fluid than in the boiling liquid nitrogen at atmospheric pressure. Since the use of liquid nitrogen under these conditions yields excellent tissue preservation, it can be recommended as an inexpensive alternative method or training procedure for helium cryofixation. 

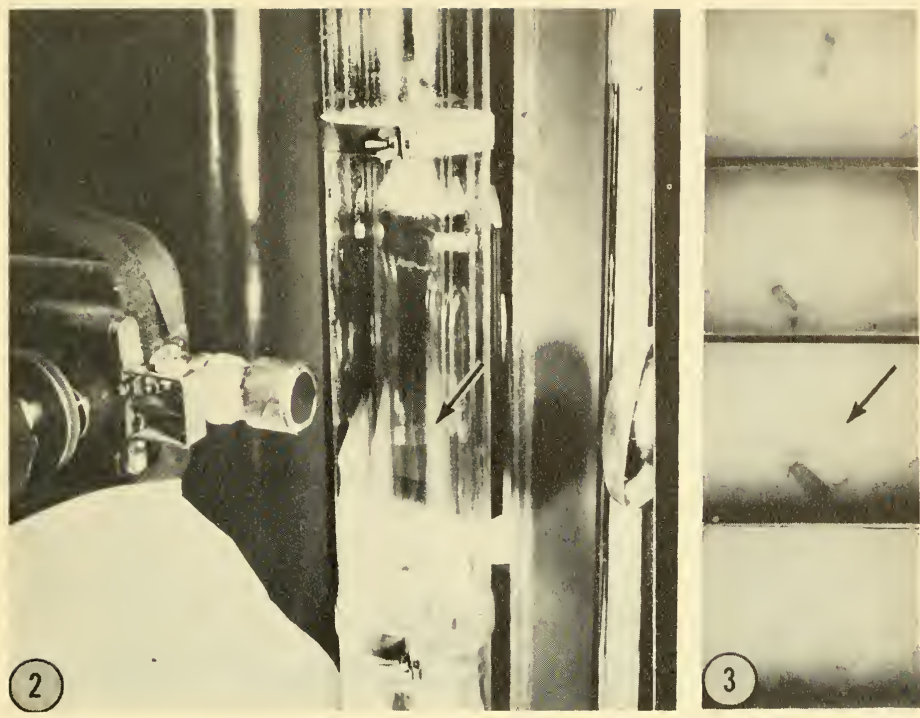

Fig. 2. Liquid helium II (arrow) in shielded Dewar at $1^{\circ} \mathrm{K}$, showing characteristic quiescent appearance. Because of the exceedingly high heat conductivity of helium II, a temperature difference between top and bottom of the liquid sufficiently great to allow the formation of vapor bubbles cannot be produced.

Fig. 3. Serial high-speed photographs showing immersion of specimen in liquid helium II without producing boiling. (Courtesy, Professor Harold E. Edgerton, Department of Electrical Engineering, M. I. T.)

Technical Requirements and Equipment for Cryofixation. In order to avoid ice-crystal and extraction artifacts during the processing cycle, which may take several weeks in certain specimens, a special "cryofixation assembly" (Fig. 4) was developed for semiautomatic freeze-substitution and photopolymerization embedding within a modified Harris refrigeration unit designed to maintain $-100^{\circ} \mathrm{C}$. The sealed specimen chamber (Fig. 5), formed by a Teflon gasket bounded by thin Plexiglas sheets and sandwiched between aluminum plates, permits regulated fluid transfer through inserted valves. This type of chamber is also useful for other types of preparations, since whole tissues can be embedded in one block and the segments removed without distortion for precise sectioning in any desired orientation. All substitution and embedding fluids were 


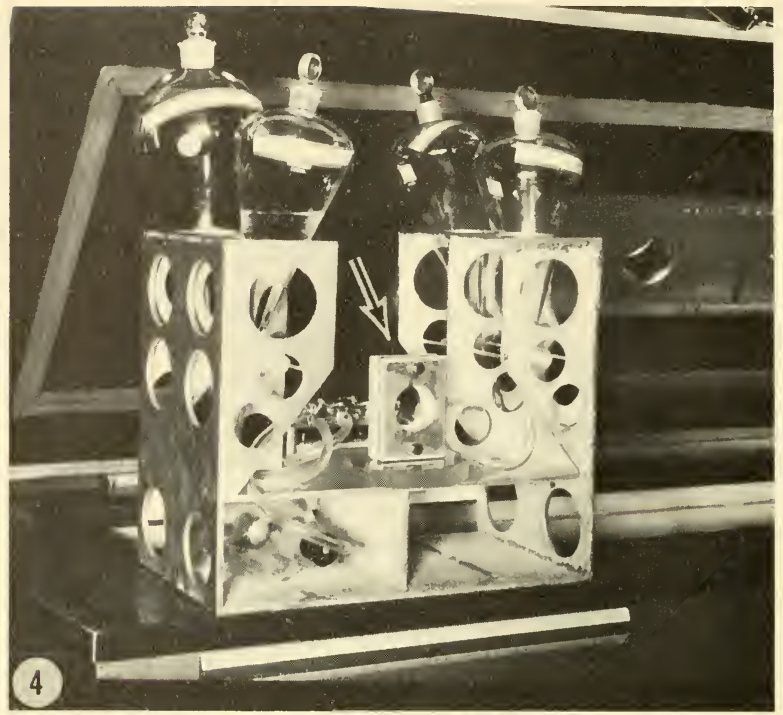

Fig. 4. Cryofixation assembly for semiautomatic freeze-substitution, infiltration of specimens, and photopolymerization embedding. After rapid freezing in liquid helium II or Freon 22-liquid nitrogen, the specimens remain in special chambers (arrow) within refrigeration unit (at $-130^{\circ}$ to $-90^{\circ} \mathrm{C}$ ), emerging ready for sectioning.

prepared in anhydrous (less than ten parts per million water content) form by passage through Linde $4 \mathrm{~A}$ molecular sieves, followed by ultrafiltration to remove suspended particles. Protective treatment (Fernández-Morán, 1952; Lovelock, 1953; Parkes, 1951) by controlled glycerination (30 to 60 per cent glycerol-veronal buffer solutions) of larger tissue specimens prior to freezing, with subsequent low-temperature transfer over alcohol or methylcellosolve mixtures to the acrylic monomers, was found to be essential for adequate preservation of fine structure (Fernández-Morán, 1959a, 1959 c). Osmium cryofixation used mainly in this study involved (1) freezing of the tissues in liquid helium Il (or in Freon 22-liquid nitrogen at $-150^{\circ} \mathrm{C}$ for control purposes); (2) freeze-substitution at $-130^{\circ}$ to $-80^{\circ} \mathrm{C}$ in 2 per cent osmium tetroxide in acetone-ethyl chloride mixtures; (3) impregnation at $-75^{\circ} \mathrm{C}$ in mixtures of methyl acrylate and butyl-methyl methacrylate monomer; and (4) 


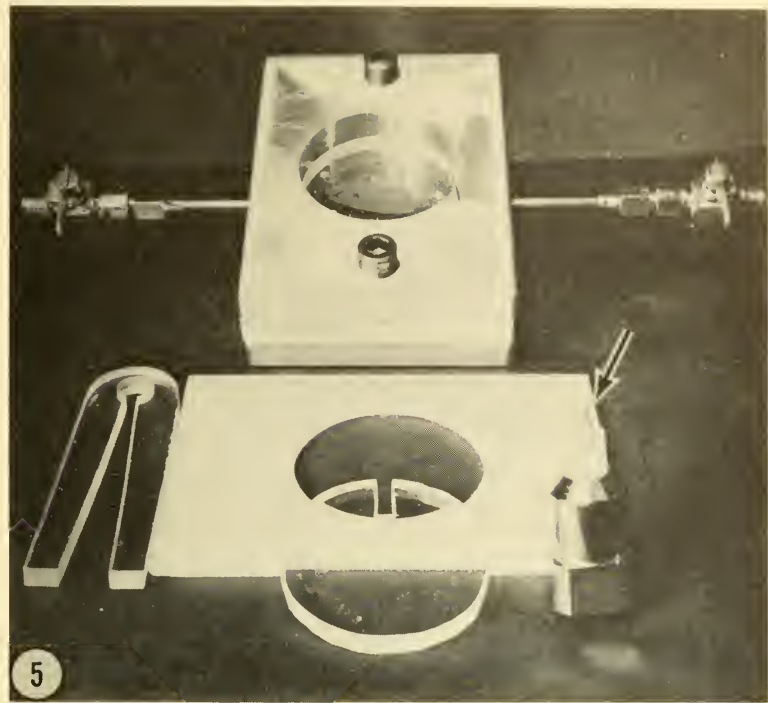

Fig. 5. Specimen chamber for cryofixation preparations, consisting of Teflon gasket bounded by thin Plexiglas sheets sealed between aluminum plates. Polymerized specimens are easily removed and mounted for ultrathin sectioning (arrow).

final low-temperature photopolymerization with ultraviolet light, using benzoin in 0.3 per cent concentration as a catalyst.

The control preparations included standard osmium-fixed and araldite-embedded nerve fibers and retinas, as well as specimens fixed in situ with osmium, iodine, or bromine vapors and embedded at low temperatures. Serial ultrathin sections of 100 to $300 \AA$ were prepared with a Morán-Leitz ultramicrotome equipped with a diamond knife (Fernández-Morán, 1953). The sections of "unfixed" retinas were collected on the cold liquid surfaces of certain fluorine compounds (e.g., Freon 11) to avoid contact with water. These sections were examined with an electron microbeam of very low intensity, using the double condenser of the Siemens Elmiskop I operating at 40 to $80 \mathrm{kv}$. A Siemens-Liesegang liquid nitrogen cooling device with modified shielding apertures was used in conjunction with multiple-objective apertures for low-temperature electron microscopy (Fernández-Morán, 1959c). 
Application of Histochemical and Autoradiography TechNiQues. Thin sections of the "unfixed" and largely undenatured specimens can be further subjected to controlled staining, extraction, enzymatic digestion, and numerous other histochemical procedures. The development of adequate high-resolution autoradiography techniques for electron microscopy would be of considerable significance for the field of ultrastructure research, since the structures revealed at the macromolecular level could then be more specifically identified and evaluated in terms of chemical composition and activity. Low-temperature preparation techniques appear to be particularly suitable for this purpose, since the radioactive tracers could be immobilized by rapid freezing in their natural position within the well-preserved tissue matrix (Fernández-Morán, 1959c). The introduction of compounds leading to the formation of silver bromide within the cells during the process of freeze-substitution represents one of the most promising approaches. In this high-resolution autoradiography method which is now being developed, the cellular matrix substitutes for the gelatin base of ordinary photographic emulsions. Low temperatures markedly reduce the fog and background without impairing the cumulative effects of the radioactivity processes on the silver halides. After exposures of the order of 2 to 8 weeks, the latent image centers are developed and enlarged at low temperatures by using gold thiocyanate (Hoerlin and Hamm, 1953) solutions. Interpretation of the images obtained with this method is still highly tentative, since the possibility that we might be dealing with "autochemograms" and other spurious effects must first be conclusively ruled out.

\section{Results}

To establish a reliable basis for evaluation of the fine structures revealed by different types of preparation procedures, the lamellar systems in the nerve myelin sheath and retinal rod outer segments of the frog and guinea pig were examined, in addition to characteristic paracrystalline granules found in the retinal pigment epithelium. Using thin sections of standard osmium-fixed and embedded preparations as controls, the effects of freezing, freeze-substitution, staining, and low-temperature polymerization were systematically investigated. 
In general, satisfactory preservation of the integrity and fine structure of lamellar systems could be achieved by rapid freezing of thin, fresh specimens or of glycerol-treated larger tissue samples, preferably with liquid helium II, followed by controlled freeze-substitution at temperatures of $-130^{\circ}$ to $-80^{\circ} \mathrm{C}$, and low-temperature polymerization. However, thin sections of this "unfixed" material showed such lability and low contrast when examined directly in the electron microscope that staining with heavy-metal salts was found to be necessary, except in the case of nuclear components which exhibit sufficient contrast and structural detail even in unstained preparations. In order to draw a more direct comparison of the new procedures with standard electron microscopy techniques, the findings presented will be confined mainly to those obtained with osmium tetroxide staining, either introduced during freeze-substitution or applied in vapor form to the "unfixed" ultrathin sections.

However, in addition to the well-preserved regions, there are still many areas in the specimens which exhibit ice-crystal artifacts, artificial rearrangements, and other complex modifications introduced by the new low-temperature niethods. Evaluation of these preparation artifacts has not only served to emphasize the limitations and pitfalls of the new techniques, but also provided valuable indications for improved preservation of the peculiarly labile hydrated state of the lipoprotein components in lamellar systems.

Fine Structure of the Nerve Myelin Sheath. Selection of the nerve myelin sheath as a standard reference system for comparative studies of lamellar fine structure was clearly indicated in view of the extensive data already available on normal and modified myelin (Fernández-Morán, 1957, 1959b; Finean, 1958; Schmitt, 1959; Schmitt et al., 1935). The correlative investigation of the preparation procedures and experimental modifications, achieved by systematic application of x-ray diffraction methods and electron microscopy (Fernández-Morán, 1959b; Fernández-Morán and Finean, 1957; Finean, 1958; Schmitt et al., 1935), has been of particular value in defining the basic structural parameters of the fresh myelin sheath. Thus, the fundamental radial repeating unit of 170 to $174 \AA$ shown in the low-angle $\mathrm{x}$-ray diffraction pattern recorded from fresh amphibian nerve (Fig. 8) corresponds to the layer spacing of the myelin sheath with an average period of 130 to $140 \AA$, as seen 
directly in electron micrographs of osmium-fixed thin sections (e.g., Figs. 9, 10). Shrinkage effects introduced by the preparation techniques account for the difference of 20 to $40 \AA$ between the two values; and from a detailed analysis carried out on the same specimen at each stage of the process, quantitative data were obtained on the dehydration and embedding artifacts (Fernández-Morán and Finean, 1957).

Against this background, which provides a reliable and detailed correlation between the organization of lamellar structures in the fresh myelin sheath and the corresponding electron microscope images, it is evident that myelin constitutes one of the best systems for a step-by-step validation of the results of the new preparation methods. In the following, we shall first consider the main artifact sources derived from dehydration and embedding at room temperature, before dealing with the ice-crystal artifacts.

Effects of Dehydration and Embedding. When the sciatic nerve from a living frog or guinea pig is rapidly frozen in Freon 22-liquid nitrogen at approximately $-160^{\circ} \mathrm{C}$, subjected to freezesubstitution in a 1 per cent osmium tetroxide-acetone mixture at $-75^{\circ} \mathrm{C}$ according to the procedure of Feder and Sidman (1958), and then embedded in methacrylate or araldite at $40^{\circ}$ to $55^{\circ} \mathrm{C}$ for ultrathin sectioning, certain modifications are regularly observed. Although the myelin sheaths of a few small fibers appear to be well preserved, most nerve fibers show extensive vacuolization of the sheath, gross distention, and other rearrangements leading to partial or complete disruption of the layers. The poor preservation of fine structure revealed by the high resolving power of the electron microscope stands in marked contrast to the well-preserved appearance under light microscope examination of a thicker section taken from the same block. The obliteration of fine structure, although caused in part by ice-crystal artifacts, is largely due to the extraction of the lipoprotein components of the layers during treatment with the methacrylate monomer, which acts as a lipid-extracting agent, particularly at embedding temperatures of $40^{\circ}$ to $55^{\circ} \mathrm{C}$. By contrast, remarkable improvement in the preservation of the myelin sheath structure is immediately noted when impregnation with the monomer and subsequent photopolymerization are carried out at temperatures of $-80^{\circ}$ to $-30^{\circ} \mathrm{C}$. These observations suggest that osmium tetroxide does not fix the lipoprotein constituents as effectively at low temperatures as at $0^{\circ} \mathrm{C}$. Low-temperature embedding is 
therefore essential for all freeze-substitution procedures, and has proved to be the most important single factor, actually making possible the systematic application of low-temperature preparation techniques to the study of the labile lamellar systems.

Earlier $\mathrm{x}$-ray and electron microscope studies of the standard preparative procedures (Fernández-Morán and Finean, 1957) had already disclosed complex modifications, including, first, a shrinkage of the myelin layer spacing during osmium fixation and alcohol dehydration, then a compensating expansion during methacrylate embedding. The significant extraction of material known to occur during fixation and dehydration (Müller, 1957) was therefore being partially masked by the methacrylate embedding. Although such a detailed analysis of the modifications introduced during fixation, dehydration, and embedding at low temperatures remains to be carried out, possible mechanisms which might be operative under these conditions are already suggested. Since the macromolecular matrix of the myelin lamellae, frozen and stiff, is thus maintained essentially immobilized during impregnation with the monomer and subsequent photopolymerization at low temperatures, the lipid extraction effects are not only considerably reduced, but the whole system is permanently "set" or fixed in its natural position by the polymerizing embedding medium before it is brought up to room temperature. In this way, major rearrangements of fine structure which might otherwise take place during the process of thawing are largely avoided; and investigation of ice-crystal artifacts can now be profitably undertaken.

Effects of Ice-Crystal Formation. The comprehensive x-ray diffraction studies carried out by Finean (1958) have shown that definite structural modifications occur after rapid freezing and thawing of nerve. In peripheral nerve, this usually results in a complete halving of the radial repeating unit and partial disorganization of the layers. The corresponding electron micrographs demonstrated characteristic changes ranging from an increased general structural breakdown of the layers to a pronounced accentuation and broadening of the intermediate line, which could be correlated with the halving of the radial repeat and abolishing of the difference factor (Fernández-Morán and Finean, 1957). These changes are brought about primarily by transient formation of submicroscopic ice crystals and associated freezing effects in the compact myelin sheath. They can therefore serve as a reference for evaluation of the results 
of ice-crystal formation and growth within ordered multilayered systems during protracted processing at low temperatures. When fresh nerve trunks are rapidly frozen and freeze-substituted at $-75^{\circ} \mathrm{C}$ and subjected to low-temperature embedding, the resulting thin sections reveal numerous large internal patches of structural obliteration which correspond mainly to regions of extensive icecrystal growth. The associated extraction, diffusion, and rearrangement artifacts are more marked after substitution in methanol than in acetone. Bearing in mind the rapid ice-crystal growth at $-70^{\circ} \mathrm{C}$ observed by Meryman (1956), it is evident that at temperatures above the critical glassy transition point of water, considered to be around $-100^{\circ} \mathrm{C}$ in tissues (Stephenson, 1956), ice-crystal growth can assume significant proportions during the prolonged freeze-substitution periods of days or weeks in certain specimens. More direct observations can be performed with a special cold stage and a fluorescence microscopy attachment, on model systems consisting of thin gelatin strips containing riboflavin-phosphate-sodium. As demonstrated by Szent-Györgyi (1957), the characteristic yellowish-green fluorescence emitted by a watery riboflavin solution under ultraviolet light is replaced by an orange phosphorescence on the formation of ice during freezing. This phenomenon can be used as a sensitive indicator of ice under certain conditions, and the riboflavin enclosed in the ice matrix serves also as a visible marker by which to follow the freeze-substitution process. Moreover, as the riboflavin dye is released from the dissolving ice, its progressive spread throughout the alcohol-solvent column gives an indication of the diffusion processes still operative at temperatures below $-75^{\circ} \mathrm{C}$.

Upon performing freeze-substitution at temperatures of $-120^{\circ}$ to $-90^{\circ} \mathrm{C}$, using acetone-ethyl chloride mixtures, substantial improvement in the preservation of the microstructure was noted, due to absence of larger ice-crystal formations and to the diminished extracting effects of the substituting fluids at these lower temperatures. However, at temperatures below $-90^{\circ} \mathrm{C}$, the time factor enters as an important consideration. Only thin specimens can be freeze-substituted at these temperatures within reasonable time periods; for thicker specimens, the complete processing cycle may require several weeks.

After elimination of gross ice-crystal artifacts, it is now possible to recognize some of the direct and indirect effects of submicro- 
scopic ice crystals within the layered structure. The images obtained often resemble the structural modifications in sciatic nerve described earlier (Fernández-Morán and Finean, 1957) after rapid freezing and thawing, standard osmium fixation, and methacrylate embedding. When fresh nerves were rapidly frozen in liquid helium I $\left(-269^{\circ} \mathrm{C}\right)$ and then thawed, a characteristic multiple splitting of the dense and intermediate lines was noted (Fernández-Morán and Finean, 1957), in contrast to the more homogeneous appearance and broadening of the layers encountered after rapid freezing in liquid nitrogen. In cryofixation preparations, there appears to be a more differentiated interaction of these freezing effects with the ordered lamellar substrate, leading to varying degrees of enhanced structural definition. Thus, the better preservation of tissue finestructure generally observed in specimens frozen with liquid helium II, as compared with control preparations cooled with other refrigerants, might be related to the initial formation of finer ice crystals, and to other as-yet-unknown auxiliary factors which could contribute to the stabilization of the macromolecular matrix. However, elucidation of these essential problems must await further analysis of myelin ultrastructure performed directly at liquid helium temperatures by $\mathrm{x}$-ray diffraction techniques, and pursued systematically, in combination with electron microscopy, throughout the successive preparative manipulations.

Osmium Cryofixation. When osmium cryofixation is carried out on fresh frog sciatic nerve under conditions minimizing the described artifacts, the myelin sheath in most of the outer fibers is unusually well preserved. As shown in Fig. 6, the highly regular arrangement of the concentric layers is clearly visible over extensive areas of the sheath, giving an over-all impression of precise alignment, which derives from immobilization of the straight fiber bundles in their stiffened state at low temperatures. The fine structures revealed are essentially similar to those detected in the best standard osmium-fixed preparations. The "unfixed" thin sections stain intensely with a wide variety of reagents, including uranyl acetate, phosphotungstic acid, lead hydroxide, and iodine and bromine compounds, disclosing a similar type of laminated structure in the sheath, with characteristic variations which are being investigated in greater detail. Evidence of fine structure within the layers or splitting of the dense and intermediate lines (Figs. 7-11), in addition to a more compact and dense appearance of the interspace, were con- 


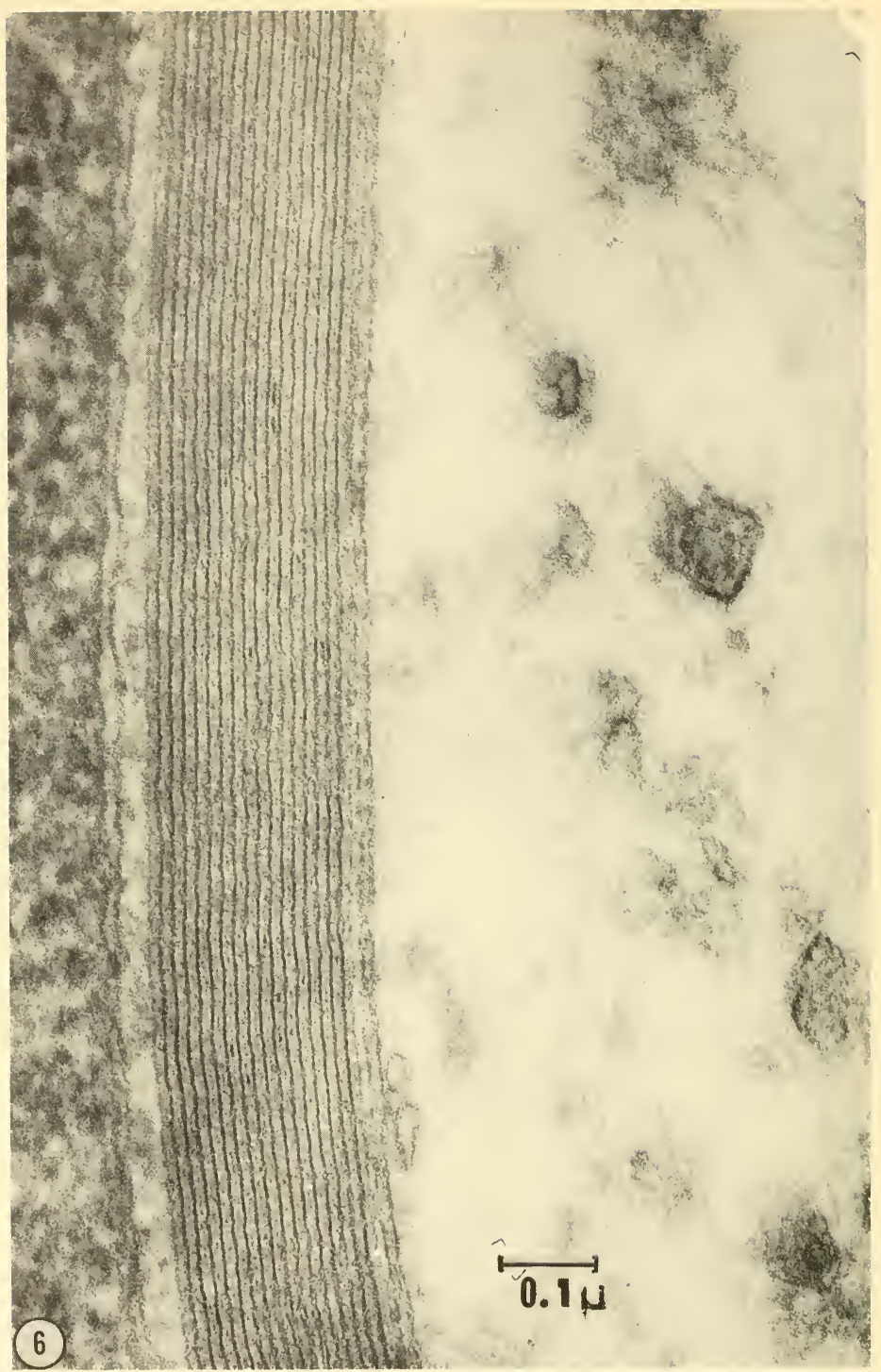

Fig. 6. Electron micragraph of myelin sheath segment from transverse section of frog sciatic nerve, showing uniform preservation of fine structure and regular arrangement of concentric layers consistently obtained with lowtemperature preparation techniques. Osmium-cryofixation preparation, low-temperature embedding $(-20 \mathrm{C})$ in mixture of methyl acrylate and butyl-methyl methacrylate. $\times 120,000$. 
sistently found in most preparations. In the axonal region, close attachment, or actual fusion, of the myelin layers with the mitochondria is occasionally observed. Unfortunately, this exceptional degree of structural integrity is confined to thin specimens and to peripheral regions of larger tissue blocks. The remaining areas show ummistakable signs of ice-crystal formation, and all transitions can be found between partial disruption of the myelin sheath structure and the zones of excellent preservation. This constitutes a serious limitation of all low-temperature techniques for normal histological
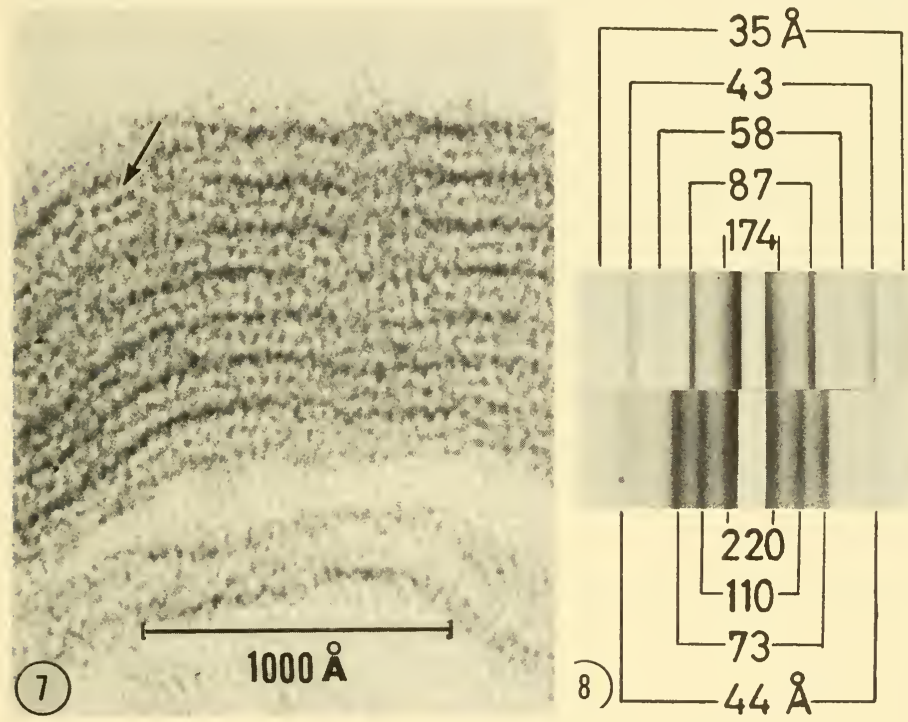

Fig. 7. Myelin sheath segment from transverse thin section of frog optic nerve, showing regular fine structure (arrow) in dense and intermediate regions of concentric layers. Nerves equilibrated in glycerol before rapid freezing in liquid helium II. Osmium-cryofixation preparation. $\times 380,000$.

Fig. 8. Low-angle $x$-ray diffraction patterns, showing the effects of glycerol treatment on the myelin sheath structure. (Above) Pattern of fresh giant toad sciatic nerve. (Below) Pattern of giant toad sciatic nerve after equilibration with 30 per cent glycerol in Ringer solution ( $24 \mathrm{hr}$ ), showing swelling of structure. Upon rapid freezing of the glycerol-treated nerve with liquid nitrogen or liquid helium I $\left(-269^{\circ} \mathrm{C}\right)$, followed by thawing, a substantially unaltered $x$-ray diffraction pattern was obtained, in contrast to the untrected nerve, in which marked modifications of the pattern were observed, indicating a partial breakdown of structure. (Experiments carried out in collaboration with Dr. J. B. Finean in 1957.) 


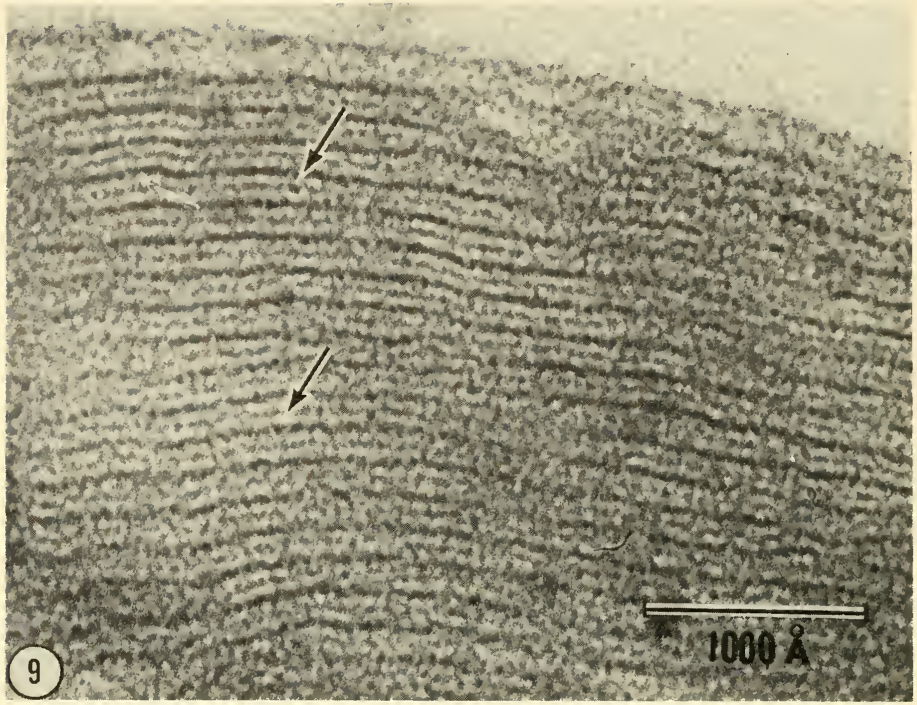

Fig. 9. Myelin sheath segment from transverse thin section of frog sciatic nerve, showing granular fine-structure (arrows) in dense and intermediate lines of concentric layers. Nerves equilibrated in glycerol before rapid freezing in liquid helium II. Osmium-cryofixation preparation. $\times 270,000$.

studies, and a systematic survey of the usefulness of certain protective agents like glycerol for the preservation of tissue ultrastructure therefore became one of the major practical objectives of our investigations.

Effects of Protective Glycerol Treatment on Myelin Ultrastructure. Pursuing earlier studies of the histological applications of glycerol (Fernández-Morán, 1952, 1957), a series of interesting observations has been made, demonstrating the general effects of glycerol treatment on the ultrastructure of the myelin sheath. The remarkable effects of glycerol which protect living cells against the damaging action of freezing and thawing (Harris, 1954; Parkes, 1951) have been primarily ascribed to the water-binding properties and salt-buffering potentiality (Lovelock, 1953) of this agent, in addition to its lack of toxicity and other unique features. Although numerous investigations of the effects of glycerol treatment on extra- and intracellular crystallization have been carried out with 


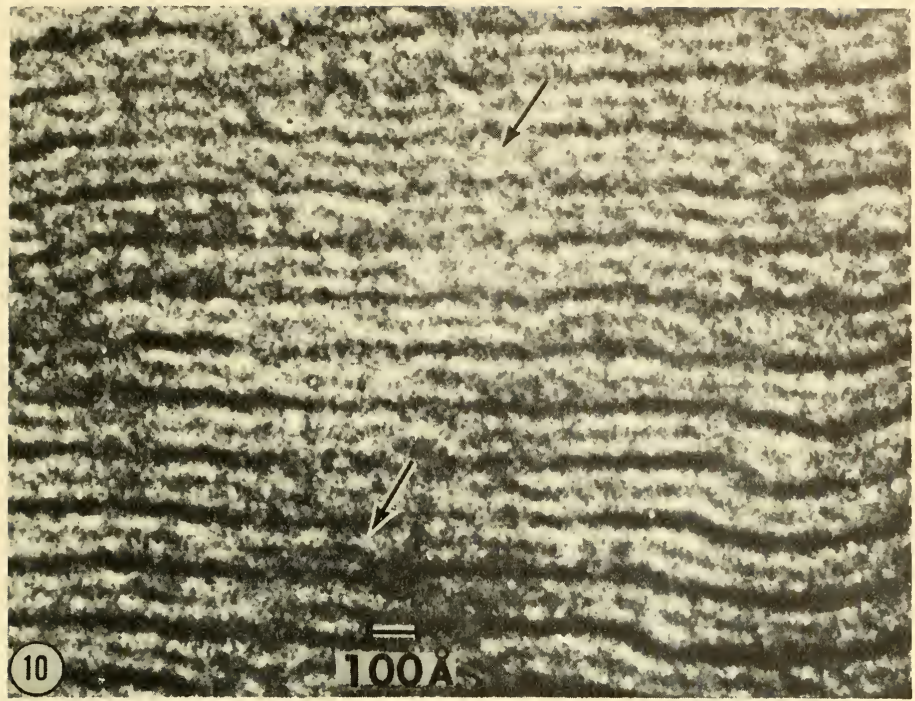

Fig. 10. High-resolution electron micrograph of myelin sheath segment from transverse thin section of frog sciatic nerve, showing splitting of intermediate lines (arrows) in concentric laminated structure, with an average period of $140 \AA$. Osmium-cryofixation preparation. $\times 500,000$.

the light microscope at low temperatures (Harris, 1954), there is no relevant information available on the interaction of glycerol solutions with the submicroscopic organization of tissues.

Preliminary (unpublished) investigations, carried out in collaboration with Dr. J. B. Finean at the Venezuelan Institute for Neurology and Brain Research in 1957, disclosed characteristic modifications of the low-angle $x$-ray diffraction pattern of fresh nerve after treatment with glycerol. The changes produced in the diffraction pattern of peripheral nerve myelin from giant toad after equilibration with 30 per cent glycerol in Ringer solution are shown in Fig. 8. Compared with the pattern of fresh nerve, which features a fundamental period of $174 \AA$, the glycerinated nerve shows general swelling of the structure with an enlarged period of $220 \AA$ and intensification of the first- and third-order reflections. The relative intensities of the x-ray reflections can be restored to approximately normal appearance by immersion in Ringer solution, but the structure remains 


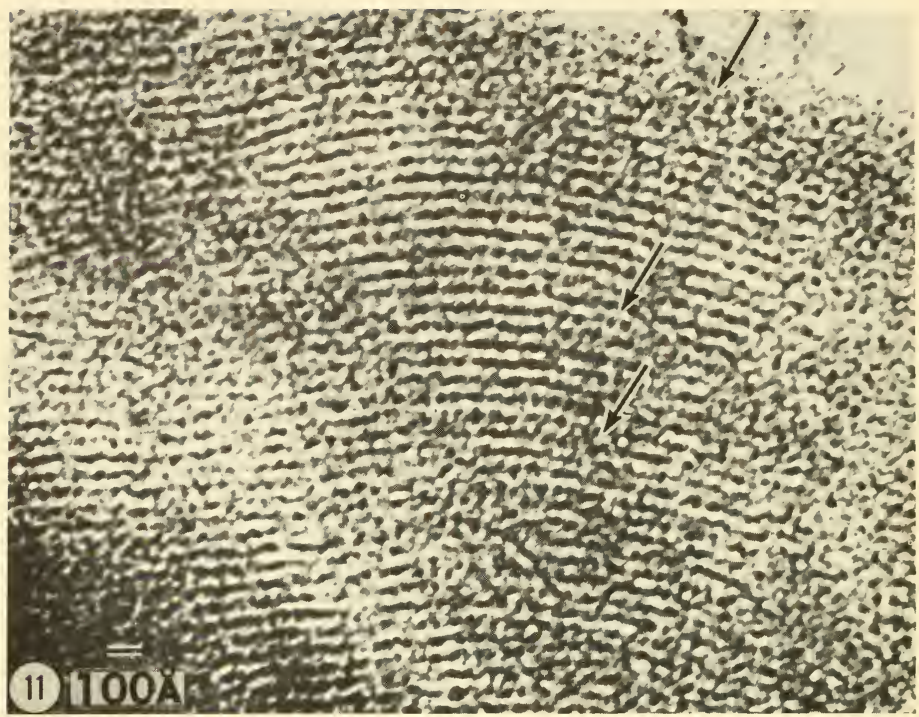

Fig. 11. High-resolution electron micrograph of myelin sheath segment from transverse thin section of frog sciatic nerve, showing granular structure of concentric layers (arrows), with a modified regular period of 50 to $60 \AA$ which probably results from enhancement of the intermediate line by treatment with bromine. Bromine-cryofixation preparation. $\times 450,000$.

expanded. The protective effect of glycerol on the myelin structure could be clearly established by showing that the diffraction pattern was not changed after rapidly freezing the glycerol-treated nerve with liquid nitrogen $\left(-195^{\circ} \mathrm{C}\right)$ or liquid helium I $\left(-269^{\circ} \mathrm{C}\right)$, then thawing. By contrast, the pattern of untreated nerve, which was subjected to the same type of freezing and thawing, exhibited pronounced modifications, indicating a partial breakdown of structure. In the course of this work it was also found that glycerinated nerves could be preserved in an essentially unaltered state for periods of several weeks at room temperature, whereas fresh nerve kept in normal physiological saline degenerated within a few days. Only when nerve is kept at $4^{\circ} \mathrm{C}$ is the disintegration process retarded, but even then the state of preservation cannot compare with that of glycerinated nerve. This objective evidence of the extensive protective action of glycerol on myelin ultrastructure emphasized 
its potential value for electron microscopy. Glycerinated nerve could not be examined directly with the techniques which were available at the time, because glycerol is not miscible in methacrylate, and the required treatment with alcohol followed by embedding at $45^{\circ} \mathrm{C}$ would have destroyed the unfixed lipoprotein structures.

However, with the development of the cryofixation techniques, the glycerol permeating the tissues could be substituted at adequately low temperatures with alcohol or, preferably, methyl cellosolve, and the tissues impregnated with the acrylic monomers and finally photopolymerized under favorable conditions which would minimize extraction and embedding artifacts. The highly encouraging results obtained with this technique have established it during the past year as the method of choice when dealing with larger tissue blocks or even whole small organs. Since equilibration with buffered glycerol solutions of increasing concentration can be performed gradually at temperatures of $-5^{\circ}$ to $-35^{\circ} \mathrm{C}$, the usual dehydration and extraction effects are markedly reduced. Once a glycerol concentration of 40 to 60 per cent has been attained, the tissue can be cooled to $-75^{\circ} \mathrm{C}$ or lower, and the entire preparation cycle completed at these "safe" temperatures. When the embedded tissue is brought up to room temperature for sectioning, the remarkable preservation of the original configuration and translucent natural colors stands in marked contrast to the opaque and artificial appearance of other preparations. Electron microscopy confirms that a high degree of structural integrity has been achieved, and it is instructive to see a cross-section through a whole "unfixed" retina perfectly intact, and free from ice-crystal artifact. In glycerinated low-temperature preparations, the lamellar systems and the nuclear structures are better preserved than the loose cytoplasmic matrix; the remaining artifacts may in fact be reduced when the equilibrium with glycerol can be refined or supplemented with a more physiological protective agent. Examination of thin sections of glycerinated nerve fibers (Figs. 7,9 ) which have been stained with osmium discloses an expanded myelin layer spacing of the order of 150 to $160 \AA$, with clear-cut indications of a granular fine structure of the intermediate and dense layers. In several areas this particulate fine structure exhibits a certain degree of regularity in the radial direction. A satisfactory correlation of the x-ray diffraction patterns with the electron micrographs must await further studies; 
but it is conceivable that the expansion of the layers may result from association of the glycerol with the water layers at the aqueous interfaces of the fundamental repeating unit of myelin. Although the glycerol effects on the myelin sheath are not completely reversible, this may still be compatible with partial recuperation of physiological activity. Thus Pascoe (1957) has shown that the rat's isolated superior cervical ganglion preparation could be protected from damage by cooling to temperatures as low as $-76^{\circ} \mathrm{C}$, as judged by the size of the transmitted action potential. Glycerol (up to 20 per cent) progressively slowed nervous conduction, but the effect was reversible (Pascoe, 1957).

Bromine Cryofixation. In contrast to the osmium-cryofixation preparations (Fig. 10), treatment with iodine or bromine reveals the presence of a more compact, granular multilayered structure (Fig. 11) with a regular period of approximately 50 to $60 \AA \AA$. Although this periodicity seems to result from enhancement of the intermediate line, as already noted in nerve fixed with $\mathrm{KMnO}_{4}$ (Fernández-Morán and Finean, 1957), there appears to be an additional staining of the less dense intermediate layer which is normally not brought out in osmium-fixed preparations. In this connection it is of interest to point out that, on the basis of his comprehensive studies of cell membrane structures, Robertson (1959) has postulated the possible existence of a polysaccharide monolayer incorporated in the outer layer of the Schwann-cell surface membrane which contributes to the formation of the intraperiod line in compact myelin. Application of more specific reagents may eventually permit reliable localization of this highly hydrated, polysaccharide component.

Fine Structure of the Myelin Layels. Previous investigations (Fernández-Morán, 1950, 1952; Fernández-Morán and Finean, 1957) had revealed that the dense myelin layers dissociate into granular or rod-shaped particles after a wide variety of treatments, including enzymatic digestion, $\mathrm{KMnO}_{4}$ fixation, and freezing and thawing of nerve. X-ray diffraction studies (Fernández-Morán and Finean, 1957) also furnished indirect evidence for the presence of a regular organization within the plane of the layers. In vitro nerve degeneration studies (Fernández-Morán, 1959b) likewise disclosed various forms of granular dissociation of the lamellae closely re- 
sembling the structures observed after enzymatic digestion. Based on these collateral data, the distinct granular structure of the dense and intermediate layers in the myelin sheath as revealed by the new low-temperature preparation techniques merits special attention, and will be considered in terms of our present concepts of the molecular organization of myelin.

It is assumed that the fundamental radial unit of the sheath is formed by two lipoprotein layers consisting of bimolecular leaflets of $67 \AA$ with interposed protein and water layers, each contributing approximately $25 \AA$ (Schmitt, 1959). The two bimolecular leaflets of mixed lipids are distinguished by a "difference factor" (Finean, 1958), which can be accounted for by assuming that in the process of myelin formation the asymmetric Schwann-cell membrane's wrapping itself around the axon (Geren and Schmitt, 1955; Robertson, 1959 ) will produce a symmetry difference in successive layers. According to Robertson (1959), each repeating unit would therefore essentially comprise two Schwann-cell membranes in contact along their outside hydrophilic surfaces to form the intraperiod line. Since rapid freezing and the described low-temperature preparation techniques directly affect the aqueous interfaces and other hydrated structures, the characteristic changes can be related in part to the regular distribution of the water layers in the myelin sheath. The intensification of the intermediate line observed after freezing and thawing (Fernández-Morán and Finean, 1957; Finean, 1958) would therefore probably result from ice formation and associated freezing effects along the highly hydrated intraperiod line. By analogy, the swelling of the myelin layers found after glycerol treatment would be produced by infiltration and binding of the glycerol within the same hydrophilic planes. In order to account for the permeability properties of the membrane, the radially oriented lipid molecules with a cross-section of $4.7 \AA$ are considered to be sandwiched between monolayers of protein with a fenestrated type of structure (Engstrom and Finean, 1958; Fernández-Morán, 1959b). Considering the postulated sieve mechanism and the fluid crystalline state of the membranes, the water constituent would again have to play an important role in the organization within the plane of the lipoprotein layers. The characteristic dissociation of the layers into rodshaped granules produced by freezing, enzymatic digestion, and glycerol treatment can be understood in terms of an underlying 
drastic alteration of the hydrated matrix, which is responsible for the ordered lateral aggregation of the radially oriented lipoprotein components.

Axon Structures. While surveying the sheath-axon relationships, it was found that a large number of the normally dense axon filaments, about $100 \AA$ in diameter, showed a characteristic "hollow" annular appearance (Fig. 12, a and b) with a central light area

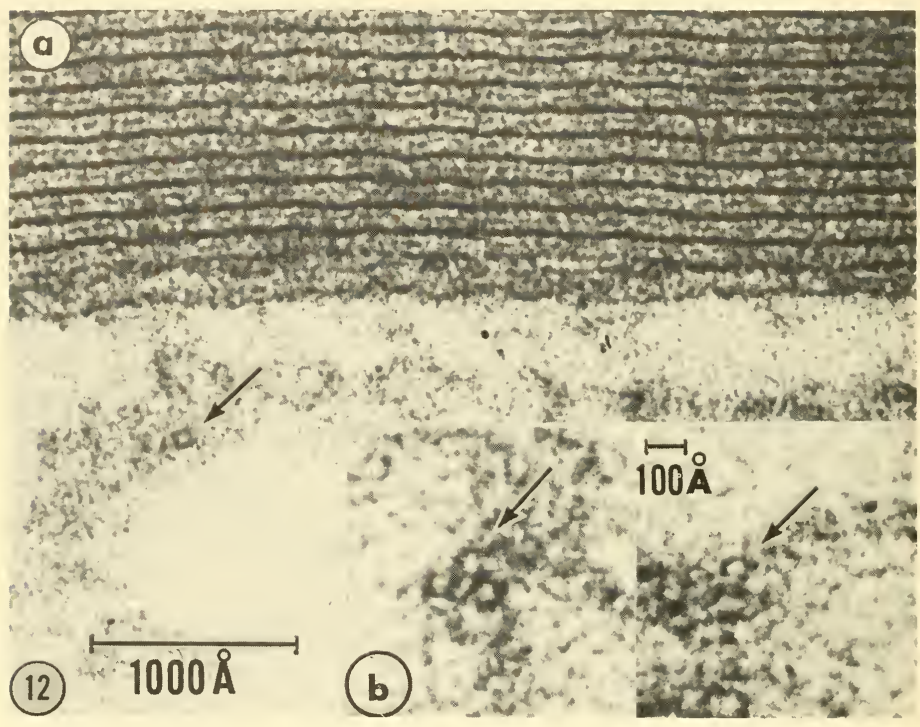

Fig. 12. Electron micrographs of myelin sheath-axon segment from transverse section of frog sciatic nerve, showing (a) fine structure of concentric layers and (b) characteristic annular appearance of cross-sectioned axon filaments which is regularly encountered in low-temperature preparations. Osmiumcryofixation preparation. (a) $\times 250,000$. (b) $\times 450,000$.

approximately $30 \AA$ in diameter. The edges of these ring-shaped elements exhibit indications of granular fine-structure which could not be clearly resolved. Although this hollow appearance of the axon filaments might be due to ice-crystal artifacts, the regularity of the cross-sectioned structures and the satisfactory preservation of the adjacent myelin sheath would favor the assumption that we are dealing with a reproducible structural pattern, corresponding to an 
equivalent configuration of the native filaments. The images recorded are similar to transverse sections of dendrites, or to the cross-sectioned tobacco mosaic virus particles described earlier (Fernández-Morán and Schramm, 1958). These observations may be of interest in connection with the extensive chemical and physicochemical studies of the macromolecular constituents of axoplasm which are now being carried out by Davison, Taylor, and Schmitt

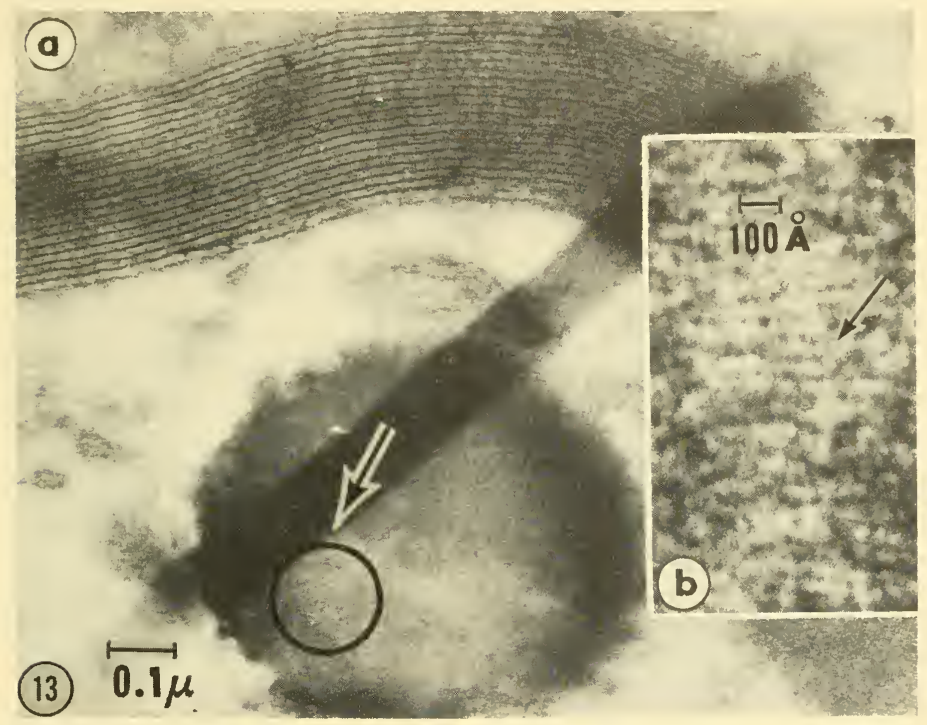

Fig. 13. Myelin sheath-axon segment from transverse section of frog sciatic nerve, showing (a) dense body frequently observed in the axon, with (b) internal layered structure exhibiting a period of 40 to $50 \AA$. Osmium-cryofixation preparation after rapid freezing in Freon 22-liquid nitrogen. (a) $\times 80,000$. (b) $\times 450,000$.

(Schmitt, 1959). Another component which is encountered in osmium-cryofixation specimens more frequently than in the standard preparations, is represented by dense bodies of $0.2 \mu$ to over $1 \mu$ in diameter. As shown in Fig. 13, many of these bodies exhibit a regular layered fine-structure with a period ranging from 30 to $40 \AA$. The bodies are more numerous in certain regions of the nerve fiber, particularly in the vicinity of the nodes. 
Fine Structure of Photoreceptors. The lamellar systems in photoreceptors are of particular interest because they represent the primary sites of visual excitation where light is converted into chemical or electrical energy. From earlier polarized-light studies (Schmidt, 1937, 1951) it had been deduced that the rod outer segments of the vertebrate retina consist of transversally arranged, thin protein layers alternating with longitudinally oriented layers of lipid molecules. Electron microscopy confirmed this conception by demonstrating that the entire rod outer segment is built up of several hundred unit disks about $150 \AA$ thick, in regular compact arrangement (Fernández-Morán, 1954; Sjöstrand, 1949, 1953). As first shown by Sjöstrand (1949), the isolated unit disks of the guinea-pig rod outer segment are about $140 \AA$ in thickness and approximately $2 \mu$ in diameter. In thin sections of osmium tetroxide standard preparations (DeRobertis, 1956; Sjöstrand, 1953), each disk is seen to correspond to two dense layers fused at their ends and enclosing a lighter space, to give a total thickness of approximately $140 \AA$. The light interspaces between the disks are considered to be filled mainly with an aqueous, ionic medium, according to Sjöstrand, who has also tentatively assumed that the 30 to $40 \AA$-thick dense osmiophilic layers are essentially of protein nature, while the 70 to $80 \AA$, less dense interspace could accommodate a double layer of lipid molecules (Sjöstrand, 1959).

Thin sections of light-adapted guinea-pig retinas which have been subjected to the osmium-cryofixation procedures (Figs. 14, 16) disclose a more differentiated picture. The rod outer segments now appear exceptionally compact, with few indications of the discontinuities frequently observed in standard preparations. The less dense interspace of each unit disk, which in standard preparations appears essentially devoid of fine structure, contains a distinct intermediate line 15 to $20 \AA$ thick (arrows, Figs. 14, 16). This intermediate line is associated with a compact granular material which gives each unit disk the appearance of greater density than is observed in the corresponding images of standard preparations (Fig. 15). The interspaces between the disks have also been reduced to barely visible gaps between the dense lines of each disk. This close apposition of two structurally asymmetric units now bears a close resemblance to the arrangement of the asymmetric unit membranes as seen in the myelin sheath and in chloroplasts (Hodge, 1959). The photoreceptor elements, which had apparently constituted an excep- 
tion to the general structural pattern, are now seen to be invested, through the presence of an intermediate line, with the same feaures common to all types of compound unit membranes (Robertson, 1959). These findings have been further confirmed by cryofixation of other types of vertebrate and invertebrate photoreceptors, using
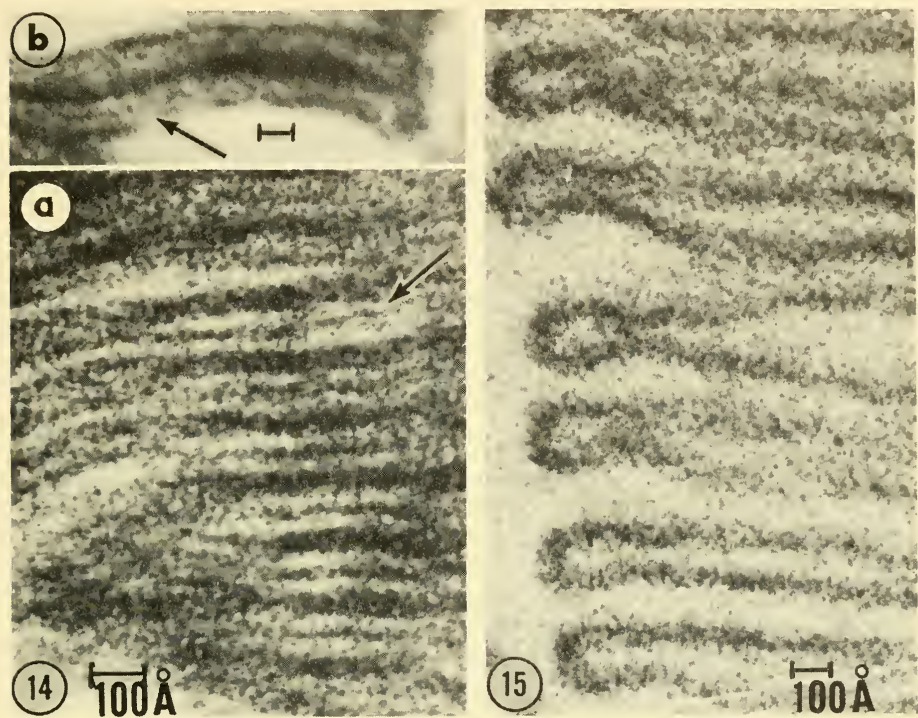

Fig. 14. High-resolution electron micrographs of rod outer segments from light-adapted guinea-pig retina frozen in liquid helium II, showing intermediate lines (arrows) and compact fine-structure of unit disks which are not observed in standard osmium-fixation preparations (see Fig. 15). (a) $\times 600,000$. (b) $\times 350,000$.

Fig. 15. External segment of light-adapted guinea-pig retinal rod, showing profiles of comparatively "empty" unit disks without intermediate lines, as commonly observed in standard osmium-fixed preparations. $\times 400,000$.

a wide variety of heavy-metal salts and other fixation procedures (Fernández-Morán, 1959a). Although the possibility of artifacts cannot be discounted here, it nevertheless appears likely that the low-temperature preparation techniques, with their absence of extraction, and other favorable features, reveal a structural array which approximates the natural state more closely than do the standard 
fixation and embedding procedures, with their concomitant extraction artifacts.

Since the photopigments form an integral part of each unit disk, it appeared worthwhile to apply the new low-temperature preparation techniques to a comparative study of dark- and light-adapted photoreceptors. Rapid freezing preserves the labile photopigments,
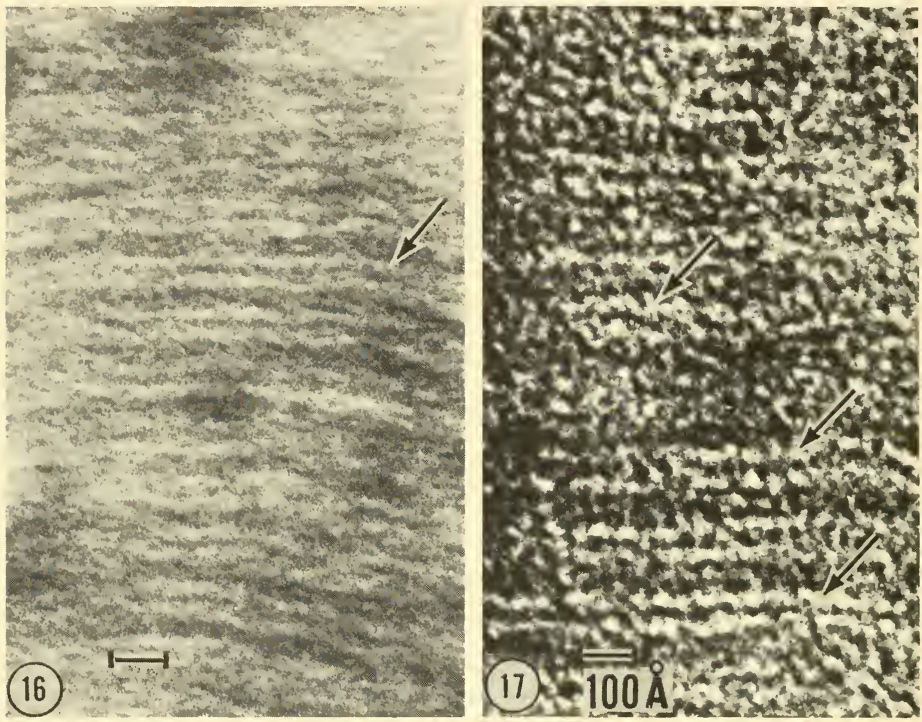

Fig. 16. External segment of light-adapted guinea-pig retinal rod, showing intermediate lines and compact fine-structure of the unit disks. Liquid helium II, osmium-cryofixation preparation. $\times 600,000$.

Fig. 17. External segment of dark-adapted guinea-pig retinal rod, showing granular and rod-shaped structures (arrows) with indications of orientation in the planes of the unit disks. Platinic chloride, osmium-cryofixation preparation after liquid helium II-freezing. $\times 600,000$.

and it was therefore hoped to detect characteristic differences in the ultrastructure of dark- and light-adapted rod disks as a basis for further studies on photopigment localization. In preliminary experiments (Fernández-Morán, 1959a), dark-adapted fresh or glycerinated guinea-pig retinas were rapidly frozen in liquid helium II or in Freon 22-liquid nitrogen, then subjected to freeze-substitution and low-temperature polymerization. When the frozen retinas were 
examined at approximately $-150^{\circ} \mathrm{C}$ in a special low-temperature stage, the characteristic salmon-pink color of the dark-adapted retinas could be observed essentially unaltered over periods of several hours. However, in the subsequent process of freeze-substitution, the red color regularly changed to a pink-yellow. Upon completion of the low-temperature photopolymerization process, the dark-adapted retinas exhibited a distinct yellow color and could be readily distinguished from the corresponding light-adapted retinas. If ultrathin sections of dark-adapted retinas are collected on inert fluids instead of water and examined in the electron microscope, using a liquid-nitrogen cold stage (Fernández-Morán, 1959a, 1959d), a characteristic electron-dense constituent is detected associated with the double layers. This component appears to be very sensitive to the effects of the electron beam, and even when a microbeam of very low intensity is used, it rapidly disintegrates. It was therefore found necessary to stain the dark-adapted retinas with platinic chloride and other heavy-metal salts introduced during the process of freeze-substitution. Under these conditions, the dark-adapted retinas exhibited a distinct yellow color which was preserved with little alteration throughout the entire preparation process. Examination of thin sections of these specimens (Fig. 17) disclosed the presence of a granular material, associated with the dense layers and the intermediate line, which seemed to exhibit a certain degree of orientation as compared with the corresponding preparations of light-adapted retinas (Fig. 16). However, these observations are still very preliminary, and much more work remains to be done before definite conclusions can be drawn. The described changes occurring in dark-adapted retinas during the process of freeze-substitution emphasize the lability of the photopigments and the need for caution in the interpretation of these images. Through a correlation of the electron microscope studies with polarization-optical analysis of the characteristic dichroism observed in dark-adapted retinal rods (Schmidt, 1951), it may eventually be possible to localize the photopigments and establish their structural relationships to the lipoprotein layers.

The photoreceptor elements of the insect eye, which consist of closely packed, thin-walled tubules approximately 400 to $500 \AA$ in diameter (Fernández-Morán, 1958), have also been examined with the new techniques. The walls of each of the tubular compartments are formed by a thin osmiophilic line, 20 to $30 \AA$ wide, associated 
with a dense layer approximately $60 \AA$ thick. In dark-adapted insect eyes, a dense, granular component with indications of orientation is seen in the outer tubular compartments, particularly after platinic chloride-osmium cryofixation. Continuation of these studies, which have already demonstrated characteristic differences between darkand light-adapted photoreceptor elements, will undoubtedly prove to be of interest in connection with the postulated models of photoreceptor structure (Wald, 1958; Wolken, 1958b).

Paracrystalline Components of the Retinal Pigment EpitheLIUM. The pigment epithelium is intimately associated both structurally and functionally with the photoreceptor elements of the vertebrate retina (Wald, 1958). Previous electron microscope studies (Porter, 1957; Yamada et al., 1958) had demonstrated the presence of characteristic lamellated components in the cytoplasm of the pigment epithelium cells. With improved preparation techniques, it has now been possible to resolve the highly regular, para-

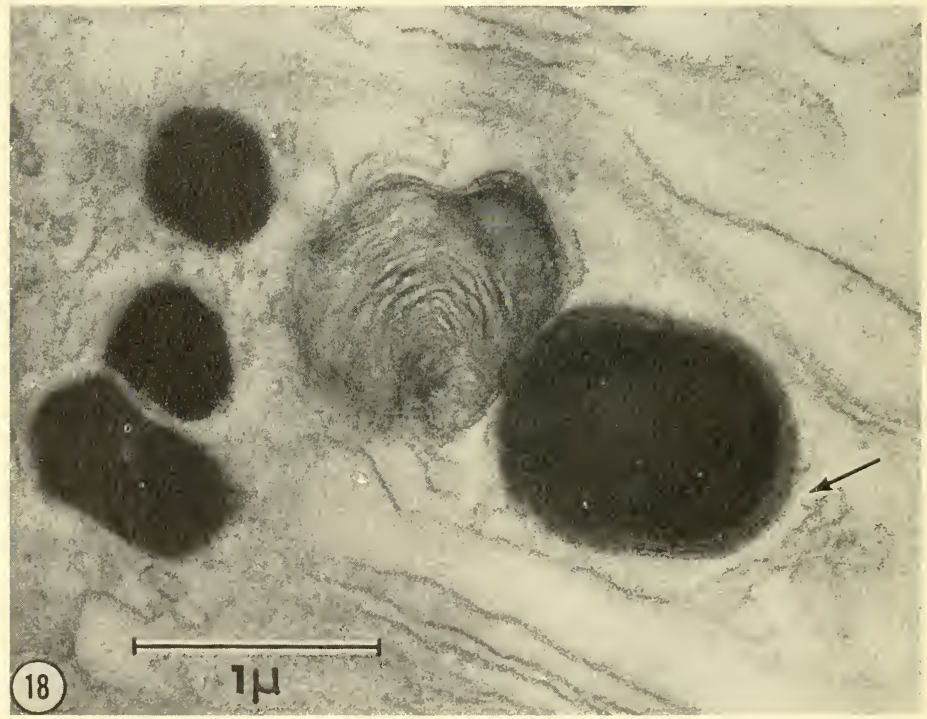

Fig. 18. Dense granules and modified mitochondria in pigment epithelium of guinea-pig retina. Certain forms of these granules are surrounded by multiple membranes (arrow) and show no distinct internal fine-structure in standard preparations. Osmium fixation-araldite embedding. $\times 30,000$. 


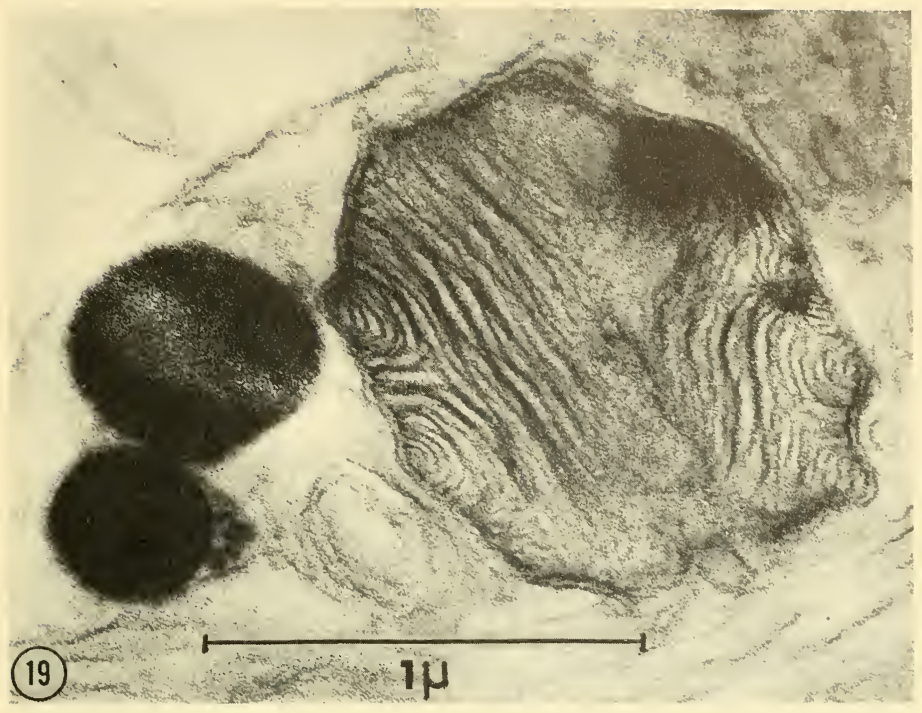

Fig. 19. Modified mitochondria in pigment epithelium cell of light-adapted guinea-pig retina, showing characteristic condensation of granular material in the layered structure. Numerous intermediate stages are found which show gradual transformation of the layered structure into the highly regular component of the paracrystalline granules. Osmium-cryofixation preparation. $\times 55,000$.

crystalline structure of certain types of dense granules within these cells and to study their interrelationship with the lamellar components of the cytoplasm. The slender, microvilli-like processes of the pigment epithelium cells envelop the retinal rod outer segments and establish intimate contact. In addition to specialized lamellar components (Porter, 1957; Yamada et al., 1958), numerous dense granules approximately $0.3 \mu$ to 1 or $2 \mu$ in diameter are found in these cells. As indicated by Yamada et al. (1958), these "fuscin granules" are extremely dense, and their internal structure is difficult to study because of their mechanical hardness, so that only small particulates or fine tubular networks could be seen.

In standard osmium-fixed and araldite-embedded preparations of the guinea-pig retinal pigment epithelium, these granules can be sectioned with the diamond knife, and exhibit an extremely compact, granular fine-structure (Fig. 18) with only occasional indica- 
tions of fine structure. In osmium-cryofixation preparations, most of these granules show a characteristic highly ordered paracrystalline arrangement (Figs. 20, 21). Depending on the plane of sectioning, a typical cross-grating pattern formed by dense round or polygonal structures 30 to $40 \AA$ in diameter with a regular spacing of 50 to $60 \AA$ is seen; or line patterns with the same spacing. This type of organization, which closely resembles the paracrystalline structure de-

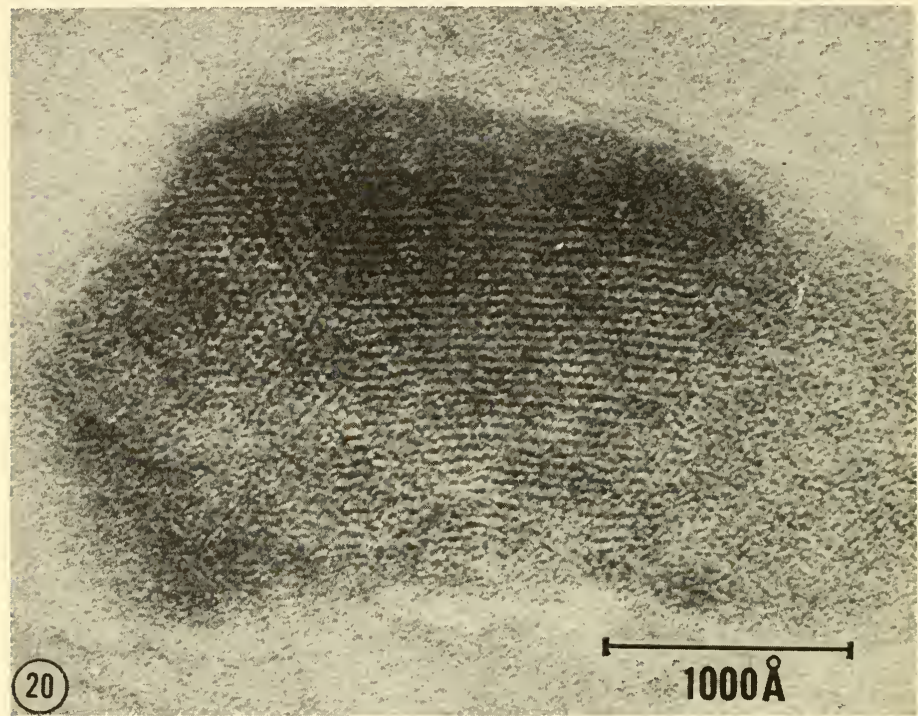

Fig. 20. High-resolution electron micrograph of ultrathin section of paracrystalline granule from pigment epithelium of guinea-pig retina, showing highly regular arrangement of the constituent dense particles, $30 \AA$ in diameter, with an average spacing of 50 to $60 \AA$. Osmium-cryofixation preparation. $\times 300,000$.

scribed in the inclusion bodies of certain insect viruses and other biological paracrystalline components, is clearly different from the compact lamellar systems of the myeloid bodies and other lamellated corpuscles with a period of 40 to $100 \AA$, as described by other authors (Porter, 1957; Yamada et al., 1958). Examination of a large number of sections discloses characteristic transitions between the mitochondria and these dense pigment granules (Fig. 18). The lamellar structure of the mitochondria shows dense patches of gran- 
ular material (Fig. 19) which gradually infiltrates the entire matrix and finally separates out into these highly ordered structures. The intermediate stages are surrounded by multiple membranes which are gradually lost. These transition stages demonstrate how a lamellar structure can transform into a more highly ordered paracrystalline system by a gradual process of condensation. Correlative biochemical studies of the paracrystalline granular components would

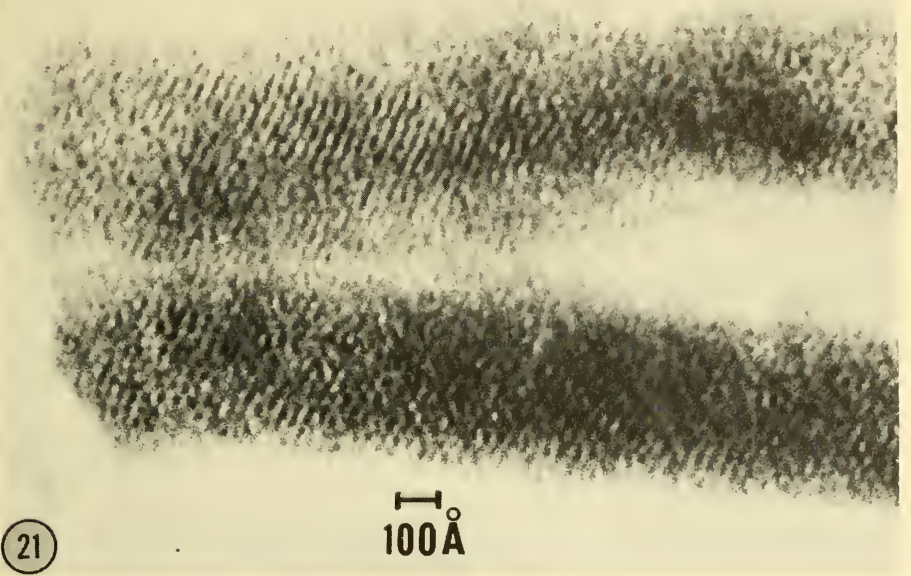

Fig. 21. Paracrystalline granule from pigment epithelium of guinea-pig retina, showing regular line or cross-grating pattern of the dense particles, depending on their orientation in the plane of sectioning. Osmium-cryofixation preparation. $\times 400,000$.

be of interest in view of the studies carried out by Wald and coworkers (Wald, 1958) which emphasize the active contribution of the pigment epithelium in the visual processes. The described paracrystalline components bear a strong resemblance to the protein crystals in the eggs of Limnea stagnalis described by Elbers (1957); and to the vitelline bodies in the eggs of Planorbis corneus. The high contrast in the macromolecular constituents of these protein crystals with a similar periodicity of $60 \AA$ is attributed to the iron content. Of particular interest are the numerous transition forms 
described by Favard and Carasso (1958), showing the mitochondrial origin of these paracrystalline granules. Further studies will contribute to a better understanding of the functional significance of this type of paracrystalline component in the cytoplasm of the different kinds of cells.

\section{Discussion}

After this survey of representative lamellar structures as revealed by different preparation methods, it may be profitable to discuss the suggested molecular organization of lamellar systems in relation to general concepts of energy transfer processes which are emerging from recent biochemical and biophysical studies.

Molecular Organization of Lamellar Systems. From a consideration of the common structural pattern revealed at the macromolecular level in the myelin sheath, photoreceptors, mitochondria, cytoplasmic lamellar complexes, and other lamellar systems, the following general picture of their molecular organization is obtained. The highly ordered lipoprotein layers derived from close packing of structurally asymmetric unit cell membranes, permeated with hydration water of possible icelike character, serve as a "paracrystalline" matrix in which assemblies of specific enzymes, associated photopigments, or specialized electron-transfer systems (Lehninger, 1959; Wolken, 1958a; Kropf and Hubbard, 1958; Szent-Györgyi, 1957 ) are precisely arranged in regular patterns.

Of special interest are the recent studies of Calvin and associates (Calvin, 1958, 1959a, 1959b) on energy-transfer processes in photosynthesis. These studies indicate that the initial process of energy reception and transfer is dependent on the specific organization of the chlorophyll and associated pigments in the highly ordered lamellar systems. Absorption of a light quantum by chlorophyll in the ground state would raise it to its lowest singlet excited state, allowing it to move around among the chlorophyll molecules by resonance transfer. The "exciton," visualized as a charge-pair which cannot move separately, migrates as an electron and "hole" through the oriented array of chlorophyll molecules. The conjugated carotenoid molecules would act as conductors of electrons, while the lipids in the chloroplasts would function as an insulator permitting a separation of charges when the electrons are "trapped" in suitable places of low potential energy on one side of the layer and the 
"holes" on the other. According to the proposed scheme (Calvin, 1959a), "the exciton is converted into conductive carriers, these into chemical radicals, and the chemical radicals lead to stable chemicals" (p. 161). Direct experimental evidence for the existence of trapped electrons and holes has been provided by recording electron-spin resonance signals from illuminated whole chloroplasts at temperatures as low as $-150^{\circ} \mathrm{C}$ (Calvin, 1959a, 1959b).

Electron-spin resonance techniques are being extensively applied to detect the presence of free radicals in enzymatic reactions, and of other intermediates with unpaired electron-spin in biological systems. However, these techniques lack the necessary sensitivity for adequate detection of the small, steady-state concentrations of free radicals in many biological processes, and for a specific localization within the complex lamellar systems. As pointed out earlier, the possibility of trapping and detecting free radicals within highly organized tissue components by rapid freezing with liquid helium II represents one of the major justifications for the use of extremely low temperatures in ultrastructure research.

It has been suggested that lamellar systems might have many properties in common with semiconductors (Fernández-Morán, 1957; Szent-Györgyi, 1957), although the concepts of solid-state physics are not directly applicable to the fluid-crystalline state (Fernández-Morán, 1954, 1959b). Recent physicochemical studies of water and ice by Eigen and his associates indicate that "The ('excess' and 'defect') proton-conducting H-bond systems (which are also of biological interest) show certain parallels to electronic semiconductors" (Eigen and DeMaeyer, 1959, p. 84). The semiconductor concept is now introduced from the unexpected quarter of water, the very constituent which had previously restricted the solid-state analogy. Attention is thus focused on a novel aspect of this fundamental "matrix of life" as Szent-Gyorgyi (1957) has so aptly designated water.

The Role of Hydration Water in Lamellar Systems. Water is a major component of all lamellar complexes, constituting, for example, at least 35 per cent of the myelin sheath. The water layers at the aqueous interfaces of the fundamental repeating unit in the compact myelin are considered to be about 12 to $15 \AA$ thick (Schmitt, 1959). It has'been shown (Robertson, 1959; Schmitt, 1959) that, under certain experimental conditions, much larger amounts of water can be incorporated between these aqueous interfaces, leading to con- 
siderable swelling. It is assumed that water is coordinated on the protein layer located at the aqueous surface of each bimolecular leaflet, the thickness of the water layer being largely determined by the electrical charge density at these interfaces, and by the ionic strength. As Schmitt (1959) has pointed out, these aqueous spaces between the membranes play an important role as channels for the products of metabolism, including ions, establishing contact between internal and external cellular spaces. The classic work of Teorell (1958) has demonstrated that periodic transport of water across the membranes and electruendosmotic phenomena are also of fundamental importance in relation to the nerve mechanism.

The effects of the electrically charged lipid groups on the structure of the hydration water in these aqueous interfaces may also induce the formation of a "crystalline" arrangement resembling the "frozen" hydration sheaths postulated in protein molecules (Fernández-Morán, 1959b). According to Klotz (1958) and Klotz and Luborsky (1959), the induction of a crystalline lattice of hydration water may be ascribed to the local and long-range cooperative electric field effects of the non-polar side-chains in proteins (Frank and Wen, 1957). The general problem of proton hydration and transfer in aqueous solution is also of fundamental interest to the biologist. According to the model proposed by Eigen and DeMaeyer (1959), the proton exists as an $\mathrm{H}_{3} \mathrm{O}^{+}$ion, connected to at least three additional $\mathrm{H}_{2} \mathrm{O}$ molecules by means of hydrogen bonds to form a $\mathrm{H}_{9} \mathrm{O}_{4}{ }^{+}$ complex (Beckey, 1959) (primary hydration shell), with a proton of excessive mobility. The proton in ice behaves more like an electron than like an ion, having a mobility which is only 10 to 50 times smaller than the mobility of an excited electron in illuminated alkali halide crystals. Eigen and DeMaeyer (1959) have pointed out that these remarkable kinetic properties of protons may prove to be of important biological significance. If we assume the existence of icelike hydration shells permeating the entire lipoprotein matrix, then this high proton mobility would become an important factor to reckon with in addition to the postulated electron mobility.

Experimental Approaches for Correlation of Structure and Function. It is against this background of a far more dynamic picture involving migration of electrons, production of free radicals, and the unique kinetic properties of protons that we must view the experimental possibilities for establishing a closer correlation between ultrastructure and function in the ordered lamellar systems. 
Our criteria for preservation of biological systems must therefore be considerably extended to take into account the new range of phenomena being revealed by refined physicochemical techniques. From a hypothetical point of view, the "ideal" preparation technique for electron microscopy of biological systems would first have to achieve complete immobilization of all tissue constituents in their natural position by rapid freezing to temperatures close to absolute zero, so that even free radicals and other transient intermediates are trapped in an inert matrix and stored under conditions restricting diffusion and radical recombination. Once the submicroscopic organization of the tissue has been "frozen in" to this characteristic "solid state," a critical transformation must then be effected at the molecular level in order to change the metastable equilibrium, which was essentially determined by the water matrix, into a new, more stable phase. This induced phase transformation, which can be likened to a process of precisely controlled, heterogeneous nucleation, would ensure adequate preservation of the tissue ultrastructure over a wide enough range of temperature and other physical conditions to permit adequate manipulation during subsequent ultrathin sectioning and electron microscope examination. Such a highly idealized form of preservation does not appear to be unattainable in principle, and eventually it may be possible to make use of recent advances in polymer chemistry involving addition polymerization reactions with certain ionic catalysts for this type of controlled synthesis of ordered polymers fitted specifically within the tissue matrix at low temperature. In order to obtain the required fundamental data on the hydration state and properties of water in lamellar systems, combined application of nuclear magnetic-resonance spectrometry (Denis et al., 1957; Fernández-Morán, 1957), low-temperature electron microscopy (Fernández-Morán, 1959d), and neutron diffraction analysis may prove to be essential. Of immediate interest in connection with the potentialities of cryofixation techniques (Fernández-Morán, 1959a, 1959c, 1959d) would be an attempt to arrest sequentially the different states of activity in nerve and photoreceptors (Katz, 1959; Kropf and Hubbard, 1958). As already shown by the pioneering work of von Muralt (1946, 1958), rapid freezing of excited nerve fixes the chemical status iand produces an accumulation of excitation waves. The recent investigations by Hill and his associates (Abbott et al., 1958) on heat production in nerve demonstrated a complex cycle of posi- 
tive and negative phases, indicating that the chemical changes involved are so extensive that, according to Nachmansohn (1959): "they ought to be susceptible to biochemical analysis" (p. 7). In view of the characteristic coupling between the enzymatic reactions and these ordered lamellar structures, a systematic investigation of excited nerve fibers by means of cryofixation techniques might be rewarding. In this way, it may be possible to detect at the molecular level the structural correlates of the role of the acetylcholine system in the permeability changes associated with nerve conduction, as postulated by Nachmansohn (1959) on the basis of his classic studies of the biochemical mechanisms of nerve activity. Tissue culture techniques (Hild, 1957; Peterson and Murray, 1955) should be of great operational value in connection with ultrarapid freezing because of the critical limitations imposed by the specimen size. Chemical and biochemical investigations (Folch et al., 1958) of the myelin sheath and other lamellar systems will continue to provide the frame of reference for all research in this field, making full use of the specific advantages inherent in the new techniques for the study of enzymatic activity by electron microscopy (Barrnett and Palade, 1959), high-resolution autoradiography (FernándezMorán, 1959d; Hoerlin and Hamm, 1953), and related cytochemical methods. With an integrated physiological, biochemical, and biophysical approach, we may thus hope to gain a better understanding of these fluid crystal elements which are the true "energy transducers of life."

\section{Summary}

A review is presented of the salient structural features of representative lamellar systems in the nerve myelin sheath and in photoreceptors, as revealed by high-resolution electron microscopy, using standard preparation techniques and improved low-temperature preparation methods based on rapid freezing with liquid helium II. The information obtained by correlative application of direct and indirect methods has furnished a reliable basis for evaluation of the findings obtained with the new methods. Characteristic paracrystalline granules of the retinal pigment epithelium in the guinea pig are described. The molecular organization of lamellar systems is discussed in relation to general concepts of energy transfer processes which are emerging from recent biochemical and biophysical studies. 


\section{References}

Aввотt, B. C., A. V. Hill, and J. V. Howarth. 1958. The positive and negative heat production associated with a nerve impulse. Proc. Roy. Soc. (London) B 148: 149.

Allen, J. F. 1952. Liquid Helium. In F. E. Simon, N. Kunt1, and E. Mendelssomx (eds.). Low Temperature Physics. Pergamon Press, Ltd., London. Pp. 66-93.

Barrnett, R. J., and C. E. Palade. 1959. Enzymatic activity in the M band. J. Biophys. Biochem. Cytol. 6: 163-170.

BECkEY, H. D. 1959. Massenspektrometrische Untersuchungen mit Hilfe einer Feldenissions-Ionenquelle. Z. Naturforsch. 14a: 712-721.

Broida, H. P. 1957. Stabilization of free radicals at low temperatures. Ann. N. Y. Acad. Sci. 67(9): 530-545.

Calvin, M. 1958. From microstructure to macrostructure and function in the photochemical apparatus. In The Photochemical Apparatus: Its Structure and Function. Brookhaven Symposia in Biol. No. 11: 160-180.

Calvin, M. 1959a. Free radicals in photosynthetic systems. Revs. Modern Phys. 31: 157-161.

Calvin, M. 1959b. Energy reception and transfer in photosynthesis. Revs. Modern Phys. 31: 147-156.

Denis, P., A. Csaki, M. Delco, J. Sprenger, H. Fernández-Morán, and IV. RaivyLER. 1957. Spectromètre à résonance magnétique nucléaire utilisant des transistors. Arch. sci. (Geneva) 10:223.

DeRobertis, E. 1956. Electron microscope observations on the submicroscopic organization of retinal rods. J. Biophys. Biochem. Cytol. 2: 319-329.

EIGEN, M., and L. DeMAeYer. 1959. Hydrogen bond structure, proton hydration, and proton transfer in aqueous solution. In VV. J. HaMer (ed.). The Structure of Electrolytic Solutions. John Wiley \& Sons, Inc., New York. Pp. 64-85.

Elbers, P. F. 1957. Electron microscopy of protein crystals in ultrathin sections of the egg of Limnea stagnalis. Koninkl. Ned. Akad. Wetenschap Proc. B 60: 96-98.

Engström, A., and J. B. Finean. 1958. Biological Ultrastructure. Academic Press, Inc., New York.

Favard, P., and H. Carasso. 1958. Origine et ultrastructure des plaquettes vitellines de la planorbe. Arch. d'anatomie microscopique et de morphologie expérimentale $47(2): 211-234$.

Feder, N., and R. L. Sidman. 1958. Methods and principles of fixation by freezesubstitution. J. Biophys. Biochem. Cytol. 4: 593-600.

Fernández-Morán, H. 1950. An electron microscope study of rat and frog sciatic nerves. Exptl. Cell Research 1: 309-340.

Fernández-Morán, H. 1952. The submicroscopic organization of vertebrate nerve fibres. Exptl. Cell Research 3: 282-350.

Fernández-Morán, H. 1953. A diamond knife for ultrathin sectioning. Exptl. Cell Research 5: 255.

Fernández-Morán, H. 1954. The submicroscopic structure of nerve fibres. Progr. in Biophys. and Biophys. Chem. 4: 112.

Fernández-Morán, H. 1957. Electron microscopy of nervous tissue. In E. Richter (ed.). Metabolism of the Nervous System. Pergamon Press, Ltd., London.

Fernández-Morín, H. 1958. Fine structure of the light receptors in the compound eyes of insects. Exptl. Cell Research Suppl. 5: 586.

Fernández-Morán, H. 1959a. Electron microscopy of retinal rods in relation to localization of rhodopsin. Science 129: 1284.

Fernández-Morín, H. 1959b. Fine structure of biological lamellar systems. Revs. Modern Phys. 31: 319-330.

Fernández-Morán, H. 1959c. Cryofixation and supplementary low-temperature preparation techniques applied to the study of tissue ultrastructure. Proc. 17th Ann. Meeting, Electron Microscope Society of America. J. Appl. Phys. 30(12): 2038. 
FERnandez-Morán, H. 1960. Low-temperature preparation technique for electron microscopy of biological specimens based on rapid freezing with liquid helium II. In Conference on Freezing and Drying of Biological Materials. N. Y. Acad. Sci. 85: 689-713.

Fernandez-Morán, H., and R. Brown. 1958. Submicroscopic organization and function of nerve cells. Exptl. Cell Research Suppl. 5: 1-644.

Fernández-Morán, H., and J. B. Finean. 1957. Electron microscope and low-angle $\mathrm{x}$-ray diffraction studies of the nerve myelin sheath. J. Biophys. Biochem. Cytol. 3(5): 725-748.

Fernandez-Morán, H., and G. Schramm. 1958. The structure of tobacco mosaic virus as revealed in ultrathin sections by electron microscopy. Z. Naturforsch. $13 \mathrm{~b}: 68-71$.

Finean, J. B. 1958. X-ray diffraction studies of the myelin sheath in peripheral and central nerve fibres. Exptl. Cell Research Suppl. 5: 18.

Finean, J. B., F. S. Sjöstrand, and E. Steinman. 1953. Submicroscopic organization of some layered lipoprotein structures. Exptl. Cell Research 5: 557.

Folch, J., M. LeEs, and S. CARr. 1958. Studies of the chemical composition of the nervous system. Exptl. Cell Research 5: 58-71.

Fox, T. G., B. S. Garrett, W. E. Goode, S. Gratch, J. S. Kincaid, A. Spell, and J. D. Stroupe. 1958. Crystalline polymers of methyl methacrylate. J. Am. Chem. Soc. 80: 1768-1769.

Frank, H. S., and W-Y, WEN. 1957 Structural aspects of ion-solvent interaction in aqueous solutions. A suggested picture of water structure. Discussions Faraday Soc. 24: 133-140.

Geren, B. B., and F. O. Schmitr. 1955. Electron microscope studies of Schwann cell and its constituents with particular reference to their relation to the axon. In Symposium on Fine Structure of Cells. Interscience Publishers, Inc., New York. P. 251.

Gersh, I., and J. L. Stephenson. 1954. Freezing and drying of tissues for morphological and histochemical studies. In R. J. C. HARris (ed.). Biological Applications of Freezing and Drying. Academic Press, Inc., New York. Pp. 329-384.

Goldsmith, T. H., and D. E. Philpotr. 1957. The microstructure of the compound eyes of insects. J. Biophys. Biochem. Cytol. 3(3): 429.

Harris, R. J. C. (ed.). 1954. Biological Applications of Freezing and Drying. Academic Press, Inc., New York.

Hicd, W. 1957. Myelogenesis in cultures of mammalian central nervous tissue. $Z$. Zellforsch. u. mikroskop. Anat. 46: 71-95.

Hodge, A. J. Fine structure of lamellar systems as illustrated by chloroplasts. Revs. Modern Phys. 31(2): 331-341.

Hoenlin, H., and F. A. Hamm. 1953. Electron microscopical studies of the latent image obtained by exposures to alpha particles, x-rays, and light. J. Appl. Phys. 24(12): 1514 .

Katz, B. 1959. Nature of the nerve impulse. Revs. Modern Phys. 31(2): 466-474.

Klein, R, and M. D. Scheer. 1958. The reaction of hydrogen atoms with solid olefins at $-195^{\circ}$ C. J. Phys. Chem. 62: 1011.

Klotz, I. M. 1958. Protein hydration and behavior. Science 128: 815.

Klotz, I. M., and S. W. Luborsky. 1959. The binding of organic ions by proteins. Effect of changes in solvent and their implications as to the nature of the complexes. J. Am. Chem. Soc. 81: 5119-5124.

Kropf, A., and R. Hubbard. 1958. The mechanism of bleaching rhodopsin. Ann. N. Y. Acad. Sci. 74: 266-280.

LeHininger, A. L. 1959. Respiratory-energy transformation. Revs. Modern Phys. 31: $136-146$.

Lovelock, J. E. 1953. The mechanism of the protective action of glycerol against haemolysis by freezing and thawing. Biochim. ot Biophys. Acta 11:28-36.

Lumisden, C. E. 1957. Aspects of the chemistry of the myelin and sheath cell complex. In Modern Trends in Neurology. Butterworth Publications, Ltd., London. Ser. II: 130. 
Luyet, B. J. 1957. On the growth of the ice phase in aqueous colloids. Proc. Roy. Soc. (London) B 147: 434-451.

Mendelssohn, K. 1956. Liquid helium. In S. FlügGe (ed.). Handbuch der Physik. Encyclopedia of Physics. XV. Low Temperature Physics. Springer Verlag, Berlin. Vol. 2. Pp. $370-461$.

Meryman, H. T. 1956. Mechanics of freezing in living cells and tissues. Science 124: $515-521$.

Miller, W. H. 1957. Morphology of the ommatidia of the compound eye of Limulus. J. Biophys. Biochem. Cytol. 3(3): 421-427.

Minkoff, G. J. 1959. Frozen free radicals. In K. Mendelssohn (ed.). Progress in Cryogenics. Academic Press, Inc., New York. Vol. 1. Pp. 139-178.

MülleR, H. R. 1957. Gefriertrocknung als Fixierungsmethode an Pflanzenellen. J. Ultrastruct. Research. 1: 109-137.

Nachmansonn, D. 1959. Chemical and Molecular Basis of Nerve Activity. Academic Press, Inc., New York.

Parkes, A. S. 1951. Preservation of spermatozoa, red blood-cells, and endocrine tissue. In R. J. C. Harris (ed.). Freezing and Drying The Institute of Biology, London. Pp. 99-105.

Pascoe, J. B. 1957. The survival of the rat's superior cervical ganglion after cooling to $-76^{\circ}$ C. Proc. Roy. Soc. (London) B 147: 510-519.

Patten, S. F., JR., and K. A. Brown. 1958. Freeze-solvent substitution technic. Lab. Inv. 7: 209.

Peterson, E. R., and M. R. Murray. 1955. Myelin sheath formation in cultures of avian spinal ganglia. Am. J. Anat. 96(3): 319-346.

Porter, K. R. 1957. The submicroscopic morphology of protoplasm. Harvey Lectures Ser. 51 (1955-1956): 175.

Robertson, J. D. 1959. The ultrastructure of cell membranes and their derivatives. Biochem. Soc. Symposia (Cambridge, England) 16: 3-43.

SснміDт, W. J. 1937. Die Doppelbrechung von Karyoplasma, Zytoplasma und Metaplasma. Gebrüder Borntraeger, Berlin.

SснміDт, W. J. 1951. Polarisationsoptische Analyse der Verknüpfung von Proteinund Lipoidmolekeln, Erläutert am Aussenglied der Sehzellen der Wirbeltiere. Pubbl. staz. zool. Napoli 23: 158-183.

Schmit, F. O. 1944. Tissue ultrastructure analysis. In O. Glasser (ed.). Medical Physics. Year Book Publishers, Chicago. P. 1586.

Schmit, F. O. 1959. Molecular organization of the nerve fiber. Revs. Modern Phys. 31(2): 455-465.

Schmitt, F. O., R. S. Bear, and G. L. Clark. 1935. X-ray diffraction studies on nerve. Radiology 25: 131 .

Simpson, W. L. 1941. An experimental analysis of the Altmann technic of freezingdrying. Anat. Record 80: 173.

Sjöstrand, F. S. 1949. An electron microscope study of the retinal rods of the guinea pig eye. J. Cellular Comp. Physiol. 33: 383.

SJöstrand, F. S. 1953. The ultrastructure of the outer segments of rods and cones of the eye as revealed by the electron microscope. J. Cellular Comp. Physiol. 42: $15-44$.

Sjöstrand, F. S. 1959. Fine structure of cytoplasm. Revs. Modern Phys. 31(2): 301318.

SJöstrand, F. S., and R. F. BAKER. 1958. Fixation by freezing-drying for electron microscopy of tissue cells. J. Ultrastruct. Research 1: 239-246.

Stephenson, J. L. 1956. Ice crystal growth during the rapid freezing of tissues. J. Biophys. Biochem. Cytol. Suppl. 2(4): 45-52.

Szent-Györgyi, A. 1957. Bioenergetics. Academic Press, Inc., New York.

TeOrelf, T. 1958. Transport processes in membranes in relation to the nerve mechanism. Exptl. Cell Research Suppl. 5: 83-100.

von Muralt, A. 1946. Die Signalübermittlung im Nerven. Birkhäuser, Basel.

von Muralt, A. 1958. The role of thiamine in nervous excitation. Exptl. Cell Research Suppl. 5: 72-79. 
WALD, G. 1958. Photochemical aspects of visual excitation. Exptl. Cell Research Suppl. 5: 389.

WolkEN, J. J. 1958a. The chloroplast structure, pigment, and pigment-protein complex. In The Photochemical Apparatus: Its Structure and Function. Brookhaven Symposia in Biol. No. 11: 87-100.

Wolken, J. J. 1958b. Photoreceptor structures. Ann. N. Y. Acad. Sci. 74: 164-181.

Yamada, E., K. Tokuyasu, and S. Iwaki. 1958. The fine structure of retina studied with electron microscope. 11. Pigment epithelium and capillary of the choriocapillary layer. J. Electronmicroscopy (Chiba) 6: 42-46.

\section{DISCUSSION}

\section{A. C. Giese, H. Fernández-Morán}

Dr. Giese (Stanford University): For what biological problems other than the ones you have mentioned are the techniques you describe likely to be especially useful?

Dr. Fernández-Monán: The described cryofixation and related lowtemperature preparation techniques have been used primarily for the study of biological lamellar systems and of nuclear structures. Although they are still in an early stage of development, the following fields of application appear to be the most immediate ones:

(1) The study of nuclear structures. At low temperatures, and with the described techniques, nuclear structures appear to be better preserved than by conventional procedures. Combined staining, selective extraction, and enzymatic digestion can be carried out on these sections which have been subjected to a minimum of chemical fixation.

(2) Of particular interest are the autoradiography techniques. The preliminary attempts to increase the resolving power of autoradiography by using tritium-labeling in connection with electron microscopy should be carried further. If successful, they would permit detection of radioactive tracers at the macromolecular level; that is to say, at a resolution of 200 to $400 \AA$, and perhaps less in the case of track formation. This would be particularly useful in connection with radiotracer studies of nucleic acid components in bacteriophages and viruses for the contact radiography method. A collateral investigation could, of course, also be carried out with differential ultracentrifugation and radioautography studies. In this case, the pellet itself would be sectioned, and radioautography performed, thus considerably increasing the scope of this technique, which has already proved so powerful.

Beyond these, there are, of course, numerous other applications. The main value of the new techniques derives from the fact that we are now in a position to considerably extend the applications of high-resolution electron microscopy in the study of biological tissues. Moreover, low-temperature stage observations permit us to enter a domain which has hitherto remained unexplored. Taking into consideration that practically all observations published to date have been made with the specimen heated to relatively high temperatures within the microscope, the value of being able to cool it down becomes evident. Specific examples would be: 
(1) The study of labile components such as photopigments at relatively. low temperatures. As mentioned earlier, the possibility of making thin films of photopigments such as extracted rhodopsin, and then examining them at low temperatures with the electron microscope, appears to be quite promising.

(2) The study of tissues with their water components (hydration shells and extracellular water) retained in their native states.

Many other applications can be foreseen in connection with x-ray diffraction studies, including investigations of the parameters connected with longterm preservation of biological systems, and the possibility of exploring new phenomena which might occur at these extremely low temperatures. 



\title{
Fibrillar Systems in the Mitotic Apparatus
}

\author{
Hans A. Went ${ }^{2}$ and Daniel Mazia ${ }^{3}$
}

Macromolecules are intimately associated with all phases of cell function and, indeed, are absolutely indispensable for many. Some, such as enzymes, can apparently perform properly as individual molecules in, vitro, although within a cell they may be coupled to structural elements imparting rigorous spatial orientation to the enzymes. Small amounts can be studied profitably by virtue of their catalytic activity. Other macromolecules occur in highly oriented aggregates forming specific subcellular structures such as chromosomes, chloroplasts, mitochondria, plasma membrane, nuclear envelope, and mitotic apparatus. The lack of easily measured activity specific for these macromolecules frequently presents difficulties to experimental elucidation of their function within the aggregates. One of the first steps that must be taken to study a macromolecular aggregate is to isolate it in quantity and disperse it into its constituent molecular subunits in a uniform solution. A potential obstacle arises when observations on a dispersed system are extrapolated and applied as an explanation for the structure and function of the intact, oriented aggregate. However, we will have to content ourselves with this limitation pending improved experimental procedures.

Many cells entering division construct an elaborate structure whose unique function appears to be that of insuring equal distribution of the hereditary material to the daughter cells. The modern term "mitotic apparatus" was applied to this structure by Mazia and Dan (1952) and embraces both the achromatic and the chromatic portions of the nuclear division figure as described by Flemming (1882). The centrosomes, the spindle, and the asters constitute the achromatic portion, and the chromatic portion is now

${ }_{1}^{1}$ This work was in part supported by University of California research funds.

${ }^{2}$ Department of Zoology, Washington State University, Pullman, Washington.

${ }^{3}$ Department of Zoology, University of California, Berkeley, California. 
called the chromosomes. It is not unnatural that a structure of the beauty of the mitotic apparatus should receive so much attention from the early cytologists, and many of the classic observations made by them have not been surpassed to this day.

One of these observers who speculated about its origin was Flemming (1882), who offered two alternatives for the source of the material incorporated into the spindle fibers of the mitotic apparatus. The first alternative states that the spindle fibers arise from the pale fibers of the achromatic region of the prophase nucleus, which in turn originate from the reticulum and nucleoli of the interphase nucleus. The second alternative imagines that they are derived from the cytoplasm, growing from this into the nucleus. Other distinguished scholars have held similar views. Boveri (1887), Strasburger (1880), Wilson (1925), and Hertwig (1909) have all considered as valid the formation of the achromatic figure from pre-existing structures visible in their cytological preparations. There was general agreement that the asters were of cytoplasmic origin but considerable dissension over whether the spindle fibers were of nuclear or of cytoplasmic origin. However, either interpretation could be valid, depending upon the material under consideration. In its essential form, the precursor concept for the origin of the mitotic apparatus germinated during the fertile "Golden Era" of cytology and, after about 50 years of relative dormancy, it is being revived. Elucidation of the origin of the mitotic apparatus could provide a made-to-order system in which to examine the completely reversible aggregation of molecular units into a transient structure of unquestionable significance to the cell. Important to this study is the synchronization that exists between the appearance of the mitotic apparatus and other cell activities, which provides the experimenter with a convenient clock.

The problem of the molecular origin of the mitotic apparatus could not be investigated directly until Mazia and Dan (1952) demonstrated that this structure could be freed of its investing cytoplasm in large numbers from a population of dividing sea-urchin eggs. One could now inquire experimentally into the possible modes of origin of the mitotic apparatus. Two basically different ones at opposite extremes were envisaged. One of these views the formation of the mitotic apparatus as the result of the de novo synthesis from small, non-specific units such as amino acids or small polypeptides. These may arise from the earlier breakdown of some storage pro- 
teins or be present in a pool. At the other extreme, we may imagine the existence in the cytoplasm of preformed molecular subunits which are assembled into the definitive mitotic apparatus with little or no modification in their structure. The latter will be referred to as the "precursor concept." It does not differ basically from views held by the early cytologists, except at the level of organization of the precursor units. They thought in terms of the rearrangement of microscopically detectable structures present in their cytological preparations, while we look upon the precursor units as being of macromolecular dimensions. The problem, therefore, was to distinguish a net synthesis, on the one hand, from the spatial rearrangement of pre-existing structures, on the other. The advent of the technique for obtaining isolated mitotic apparatus suggested an experimental design which could decide between these two extremes. Immunology offered potentially a suitable method for attempting to identify some component of the unfertilized egg with a structural component of isolated mitotic apparatus. If the mitotic apparatus were formed de novo, it would be reasonable to expect the complete absence of its structural constituents from the unfertilized egg. The precursor concept, as stated earlier, would predict the existence in the mitotic apparatus of molecules identical to a species present in the unfertilized egg.

The antigen-antibody reaction is one of the most specific chemical reactions known (Landsteiner, 1945), but this in itself did not provide a suitable means to identify with each other the same molecular species from different sources. An added complication was the expected heterogeneous nature of the reactants. The gel-diffusion technique, as originated by Oudin (1946) and modified by Ouchterlony (1949), provided the ideal solution to these dilemmas. Twodimensional gel-diffusion techniques (Oudin, 1952; Ouchterlony, 1958) can resolve very complex antigen-antibody reaction systems into a series of discrete bands of precipitate immobilized by the gel medium, and further provide an extremely sensitive means for establishing an identity or non-identity between antigens from different sources. When bands of precipitate assignable to different antigen solutions fuse or merge, instead of intersecting, the responsible antigens are considered to be immunologically identical.

What restrictions must be imposed upon an identity between two proteins as established by the fusion of two bands in gel-diffusion analysis? It seems reasonable to expect that identical antigenic 
patches are involved in the reaction between the two immunologically identical proteins and the specific antibody species participating in the precipitate. But the antigenic patches represent only very small areas on the antigen molecule relative to the entire molecule (Haurowitz, 1956; Kabat, 1957). Therefore, the combination of the antibody with its specific antigenic patch in itself could not be expected to reveal much direct information about the configuration of the remainder of the antigen molecule. Assuming that suitable precautions have been taken against coincident band formation, a very important fact is provided by the fusion of the bands. Presumably, each band develops in the gel medium along the locus characteristic for the equivalence-ratio for the specific antigen-antibody system in the gel medium. When two bands fuse, therefore, the equivalence-ratios for the reactants involved in the two bands must have been identical. This tells us that, in all probability, the same number of antigenic patches were present on each antigen molecule; for, had this number been different, the equivalence-ratios for each antigen-antibody complex would not have been the same. Consequently, the locus of each band of precipitate would have been different, and they would not have fused. Therefore, geldiffusion methods do more than inform us that identical antigenic patches occur on the molecules from different sources; they imply that the same number of antigenic patches are present on each molecule and that the spatial configuration of these molecules is sufficiently congruent to yield identical equivalence-ratios. In view of the previous discussion, it would appear that gel-diffusion techniques present us with the most critical criterion currently available for establishing an identity or non-identity between two proteins.

Very recent evidence (Feinberg and Grayson, 1959) provides some latitude for doubt about an identity between two proteins based solely on the fusion of bands of precipitate. It revealed that grass-pollen antigens cross-reacting with two different antisera could yield band patterns in conventional Ouchterlony gel-diffusion techniques suggesting an identity between two antigens, while a slight modification of this technique indicated that only a similarity existed rather than an identity. Any conclusions concerning an identity between two proteins, based on the fusion of bands, must be restricted within the limitations, not completely understood, inherent in the procedure itself. The term "identity" will be used throughout the remainder of the paper with this in mind. 
The investigation into the molecular origin of the mitotic apparatus was begun by preparing two basically different antisera. One antiserum was prepared against unfertilized sea-urchin egg antigens soluble in $0.1 \mathrm{M} \mathrm{KCl}$, and the other used dissolved, digitonin-isolated mitotic apparatus as the homologous antigen. These two antisera
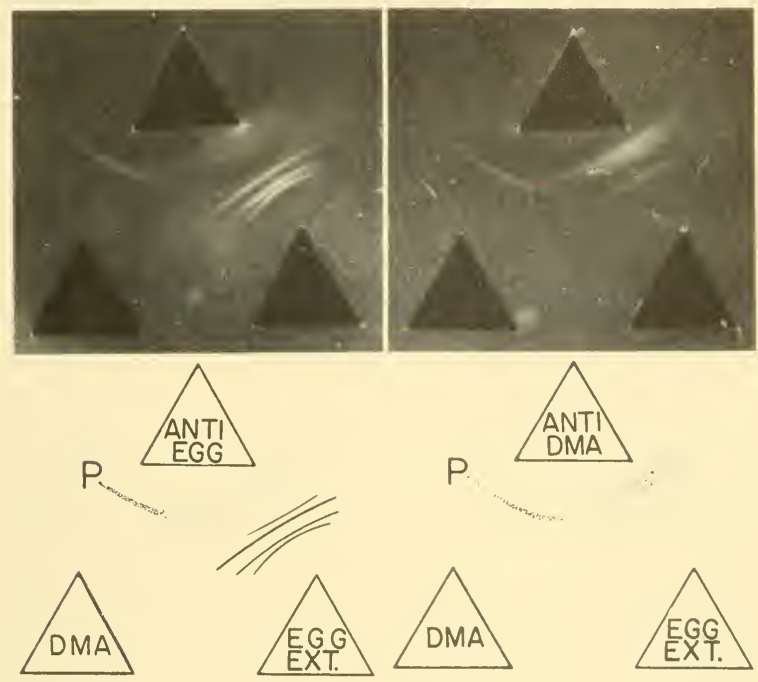

A

Fig. 1. Comparing the immunochemical behavior of antiserum to unfertilized-egg antigens (ANTI EGG) with that of antiserum to dissolved, digitoninisolated mitotic apparatus (ANTI DMA) when both were made to react with dissolved, digitonin-isolated mitotic apparatus (DMA) and an extract of unfertilized eggs (EGG EXT.). The precursor-1 band is identified by " $\mathrm{P}$ " in the line drawing corresponding to each photograph. The fusion of the only band, the P-band, ascribable to the dissolved mitotic apparatus with one assignable to the extract of unfertilized eggs indicates the presence of the precursor-1 component in both solutions.

were then made to react with soluble antigens from unfertilized eggs and from dissolved, isolated mitotic apparatus. Figure 1 shows photographs of two Ouchterlony plates, comparing the immunochemical behavior of each antiserum toward its homologous and heterologous antigens. Two important facts are established by these 
results. One is that the immunochemical behavior of the antiserum to dissolved mitotic apparatus is indistinguishable from that of the antiserum to unfertilized-egg antigens when they are both made to react with solutions of mitotic apparatus. The other is the fusion of the only band assignable to the dissolved mitotic apparatus with one of several assignable to a solution of antigens from unfertilized eggs. The antigen responsible for this band has been termed the precursor-1 component. These results are in complete agreement with the premise that the structural constituents of the mitotic apparatus are on hand in the unfertilized egg prior to the onset of the mitotic cycle. On a qualitative basis, at least, there appears to be no need for the egg to synthesize new protein species necessary for the mechanical events of mitosis, once it has left the ovary. The machinery essential for the equipartition of the hereditary material resides unassembled in the unfertilized egg awaiting the signal, brought by the penetration of a sperm, to become aggregated into the definitive mitotic apparatus.

Another very interesting inference can be drawn from the fusion of the precursor-1 component band with a band ascribable to an antigen from unfertilized eggs. When the mitotic apparatus was put into solution, it was, in all probability, disassembled by breaking the same links that were formed to assemble the precursor-1 components into the mitotic apparatis. This is the simplest interpretation for the consistent correlation of the appearance of the precursor-1 component with the loss of structural integrity by the mitotic apparatus when the latter is dissolved. It would thus seem that the dissolving of the mitotic apparatus, in terms of the end products observed, may be viewed as the reversal of the assembly process. The very mild conditions under which isolated mitotic apparatus can be dissolved (Mazia, 1959) to yield the precursor-1 component in solution preclude the peptide bond from consideration as the intermolecular link responsible for the structural integrity of the mitotic apparatus.

What type of intermolecular cross-links can account for this behavior? Mazia (1958, 1959) presents evidence, based primarily upon solubility studies of mitotic apparatus isolated by different methods, that intermolecular bridges involving sulfur-thought to be disulfide-are responsible for the polymerization of the molecular subunits into the division figure, although hydrogen-bonding cannot be entirely excluded. The involvement of sulfur-containing 
proteins in the structural organization of the mitotic apparatus is more strongly indicated by the in vivo studies of Mazia (1958) and Mazia and Zimmerman (1958). They observed that proper concentrations of mercaptoethanol ( $\mathrm{HSCH}_{2} \mathrm{CH}_{22} \mathrm{OH}$ ), a simple, penetrating SH-compound of low toxicity, applied to sea-urchin and sanddollar eggs any time before metaphase, blocked the first cleavage division. If it was applied during and subsequent to metaphase, no interference with the first cleavage division was detected. The in vivo experiments using mercaptoethanol argue strongly for the involvement of protein-SH groups during the buildup of the mitotic apparatus prior to cleavage, but do not give much insight into the actual mechanism involved. The in vitro observations on the solubility characteristics of mitotic apparatus isolated from ethanolpreserved material by the digitonin method (Mazia, 1958) favor the interpretation that some intermolecular disulfide bonds may be responsible for maintaining the spatial orientation of the "precursor" molecules in the mitotic apparatus.

The speculation that the reversible conversion of sulfhydryl groups to intermolecular disulfide linkages performs an essential role in the construction and function of the division figure finds support from other investigators. Some cytochemical studies for sulfhydryl groups in sea-urchin eggs during the first cleavage division were made by Kawamura and Dan (1958). Using Bennett's reagent, they observed that the cytoplasm and nucleus of the unfertilized egg did not stain, but 3 minutes after fertilization, they detected an increased general stainability. At metaphase, the entire mitotic figure of one species of sea urchin was intensely and uniformly stained, contrasting sharply with the less strongly stained cytoplasm. In early anaphase, the traction fibers were very intense, while the interzonal region and centrospheres took up much less of the stain. Little change in the astral rays was evident. Immediately after cleavage, the staining character of the cytoplasm of the blastomeres was essentially the same as that observed for the unfertilized egg; that is, it was unstained. Shimamura et al. (1957) have stained the mitotic apparatus for both sulfhydryl and disulfide groups, in parallel, simultaneous experiments. Their photographs clearly reveal that the regions stained for sulfhydryl groups usually complement the areas stained for disulfide groups at given stages. This was most evident in the anaphase and early telophase figures, in which the asters stained only lightly for sulfhydryl groups but very intensely 
for disulfide groups. At anaphase, the traction fibers stained strongly for both groups, while the interzonal fibers only appeared to contain the sulfhydryl groups in detectable amounts.

Studies designed to reveal quantitative fluctuations in the sulfhydryl and disulfide groups associated with protein and non-protein compounds have been performed on populations of cells at various stages of mitotic and meiotic divisions. The findings most often quoted are those of Rapkine (1931) on the TCA-soluble fraction of sea-urchin eggs. He observed a large steady drop in soluble sulfhydryl concentration after fertilization, followed by a rapid increase to a maximum just prior to cleavage. He felt that his results reflected variations in the glutathione content of the cells, and presented an ingenious scheme in which glutathione was oxidized, with the concomitant reduction of intramolecular protein disulfide bonds. The latter were then reoxidized into intermolecular bonds, and reduced glutathione was recovered. This was presumably related to the buildup of the mitotic apparatus. Recently, Neufeld and Mazia (1957) reinvestigated the problem of the non-protein sulfhydryl cycle in Strongylocentrotus purpuratus, but were unable to corroborate Rapkine's data. Since then some very careful work by Sakai and Dan (1959), again on sea urchins, uncovered a possible explanation for this discrepancy. By carefully duplicating the extraction procedure of Rapkine, they substantiated his original observations, but found that the TCA-soluble material responsible for the fluctuations in sulfhydryl was not glutathione, as Rapkine had supposed, but a protein that was soluble in TCA under the conditions used by him. Plant material has also been analyzed by Stern (1956, 1958) for protein and non-protein (soluble) sulfhydryl and disulfide. He observed fluctuations in protein and soluble sulfhydryl that correlated with meiosis and microspore mitosis in both Lilium and Trillium.

Some preliminary solubility studies on the mitotic apparatus isolated directly from living material have been conducted recently in Mazia's laboratory. The data make it more difficult to accept disulfide bridges per se as the primary intermolecular links involved in maintaining the structural integrity of the mitotic apparatus. With suitable precautions, these isolated division figures can be dissolved under extremely mild conditions. They are completely dispersed in water in the $\mathrm{pH}$ range between 8.5 and 9 , while $\mathrm{KCl}$ solutions, at neutrality, of $0.5 \mathrm{M}$ concentration or greater will dis- 
solve only the fibrous components of the mitotic figure, leaving intact the centrosomes and chromosomes (Mazia, 1958). These are not the conditions usually considered appropriate for reducing disulfide bonds. Yet the success of isolating the mitotic figure from living material depends upon treating it as though it were held together by disulfide bridges (Mazia, 1959). The apparent inconsistency between the ease with which the isolated mitotic apparatus may be dissolved and the need, during the isolation procedure itself, to use an agent (dithiodiglycol) that presumably stabilizes disulfide links, remains to be resolved. There is, thus, no clear-cut experimental evidence that directly corroborates the involvement of intermolecular disulfide bridges in the structural organization of the mitotic apparatus, nor do we have convincing proof that sulfhydryldisulfide interchanges play an important role in the functional activity of the mitotic apparatus. However, we have enough data to say that protein molecules containing reactive sulfur groups participate in some manner in the structure of the mitotic figure, and we can only speculate about the manner in which the sulfur groups react with each other to confer strict spatial orientation to the molecules bearing them. The possible mechanisms of sulfhydryl-disulfide interchanges and how these may be invoked to bring about the polymerization of the precursor molecules into the mitotic figures are adequately discussed by Jensen (1959) and Mazia (1959).

Immunochemical results allow us to consider the formation of the mitotic apparatus in terms of the aggregation of precursor subunit molecules that are present in the unfertilized egg, and the endeavors of other investigators provide indirect evidence that proteins bearing reactive sulfur groups perform important roles in the orientation of the precursor molecules in the mitotic apparatus. Gross (1954) observed that extracts of Arbacia eggs contained calcium-insoluble protein and speculated on its importance to sol-gel transformations. A similar protein was subsequently detected in the soluble fraction of a $0.1 \mathrm{M} \mathrm{KCl}$ extract of unfertilized S. purpuratus eggs. This protein appeared as a clear fibrous precipitate when $\mathrm{CaCl}_{2}$ to a final concentration of $0.05 \mathrm{M}$ was added to the unfertilized egg extract. It could be redissolved by dialysis against distilled water. Some of the physical properties of this protein, which has been termed the calcium-insoluble fraction, were studied by Kane and Hersh (1959). Their results indicated a strong resemblance between this protein and the protein present in solutions of dissolved 
mitotic apparatus. They shared similar molecular weights; each contained a ribonucleotide component; they were both relatively asymmetrical molecules; and the calcium-insoluble fraction represented about 10 per cent of the total protein of the unfertilized egg. Mazia and Roslansky (1956) have estimated that the cell invests about 12 per cent of its total protein in the mitotic figure. This immediately gave rise to the speculation that the calcium-insoluble fraction was the long-sought-after precursor component, and now we had at our disposal the means to test this more directly The calcium-insoluble fraction could be purified to the extent that, when analyzed by gel-diffusion methods, it gave rise to only one distinct band in reaction with antiserum to unfertilized egg antigens. Thus it was a simple matter to see if the precursor- 1 component band was the same as the only band assignable to the calcium-insoluble fraction.

Figure 2 summarizes the essential results. When dissolved mitotic apparatus and calcium-insoluble fraction are each compared to a solution of unfertilized egg antigens, one observes that the band associated with the dissolved mitotic apparatus merges with the one assignable to the unfertilized egg antigens, but it is readily seen that this is not the same band that merges with the only one attributable to the calcium-insoluble fraction. This clearly demonstrates that the calcium-insoluble fraction bears no direct structural relationship to the mitotic apparatus and demolishes the attractive possibility that it represents the precursor protein. Although there can be little doubt about the existence of the precursor-1 component, it could not be identified with the calcium-insoluble fraction, which was the only protein fraction obtained in a homogeneous form from unfertilized eggs.

The precursor-1 component was invariably present in all solutions of mitotic apparatus isolated from preserved material by the digitonin method. These solutions were obtained by bringing an aqueous suspension of mitotic apparatus to $\mathrm{pH} \mathrm{10-10.5.} \mathrm{At} \mathrm{these}$ $\mathrm{pH}$ values, no mitotic figures were recognizable under phase contrast, while the contaminating cytoplasmic particles remained relatively unaffected by the treatment. On the other hand, $\mathrm{pH}$ values in the range 8.5-9.0 were adequate to dissolve completely the mitotic figures isolated directly from living material, while leaving many of the undesirable cytoplasmic particles intact. These could be removed by centrifugation. Such solutions also gave rise to the 
precursor-1 component band when made to react with antiserum to unfertilized egg antigens. In addition, there very frequently appeared a second band in the Ouchterlony gel-diffusion plates, suggesting that there was a second component participating in the structural aspects of the mitotic apparatus. As a rontine procedure, antiserum was prepared against solutions of mitotic apparatus isolated directly from living material, and a very unexpected result
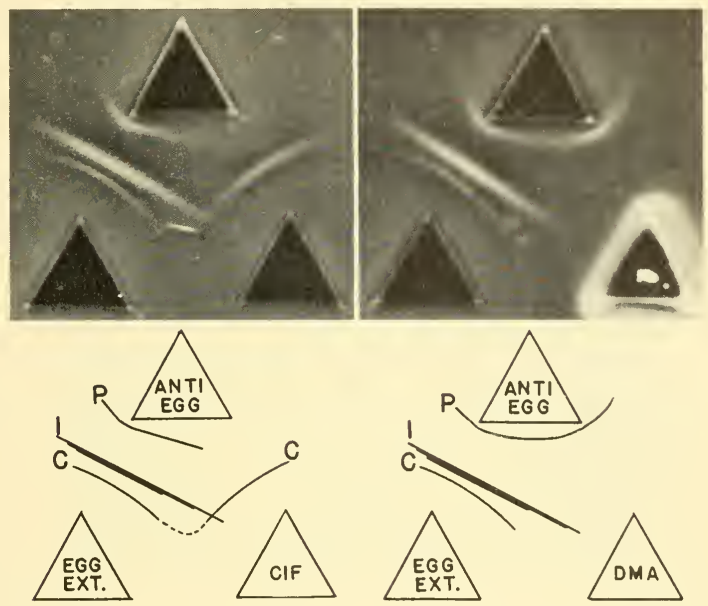

A

B

Fig. 2. Summary of the evidence indicating the absence of any direct relationship between the precursor-1 component and the calcium-insoluble fraction (CIF). The latter antigen is responsible for the C-band. (A) The C-bands were seen to fuse in the original Ouchterlony plate, but this relationship was lost in subsequent photographic procedures. (B) It can be seen that the $\mathrm{C}$-band does not fuse with the only band assignable to dissolved mitotic apparatus, revealing the lack of any simple relationship between the calcium-insoluble fraction and the precursor-1 component. The abbreviations ANTI EGG, P, EGG EXT., and DMA are the same as in Fig. 1.

was observed when this antiserum was reacted with various antigens. It could not react with the precursor-1 component but was completely specific for the second component, which will be termed the precursor-2 component. For some obscure reason, the rabbit had focused its antibody-forming capacities on only the precursor- 2 component, although the immunizing solutions had, in addition, contained the precursor-1 component. What had happened to the 
precursor-2 component in solutions of digitonin-isolated mitotic apparatus? Experiments established that the precursor-1 component was relatively insensitive to alkaline environments up to $\mathrm{pH}$ 10.5-11, the conditions under which digitonin-isolated mitotic apparatus could be put into solution, while the antigenic properties of the precursor-2 component were nearly completely destroyed in this $\mathrm{pH}$ range. Until further information is available, it will be necessary to consider the precursor-2 component essential to the structural continuity of the mitotic figure. In no way does the existence of this component necessitate any modification of the precursor concept, for it is also found in the unfertilized egg.

An important aspect of the precursor concept is the intracellular localization of the precursor-1 component in the unfertilized egg, which in turn may provide an insight into the sequence for its mobilization into the mitotic apparatus. Absorption studies were conducted, using the particulate fraction, which is arbitrarily defined as everything that can be sedimented at $60,000 \mathrm{~g}$ for $30 \mathrm{~min}$ utes from a homogenate of ethanol-preserved eggs. Extraction of the particulate fraction by various procedures, and its subsequent use to absorb antiserum, revealed that tenaciously associated with the particulate fraction was an antigen that could combine specifically with the antibodies homologous for the precursor-1 component. Evidently the precursor-1 component occurs in an insoluble form in addition to the readily extractable form, which will be referred to as the "soluble" form.

Attention was then directed toward living material, for this would lend itself more favorably to critical fractionation procedures necessary to resolve the intracellular distribution of the "precursor" components. Living, unfertilized eggs were gently dispersed in isotonic dextrose medium in a manner that avoided disrupting the yolk particles. The resultant suspension was then centrifugally fractionated, roughly into three fractions: the yolk particles, the microsomemitochondria fraction, and the supernatant to the microsome-mitochondria fraction. The yolk particles, after careful washing, were osmotically lysed and the contents analyzed by gel-diffusion methods. Numerous antigens were present, including the precursor-1 and precursor-2 components. The supernatant was qualitatively very similar to the yolk-particle lysate. The situation with the microsome-mitochondria fraction, on the other hand, was very different. This had been extracted with $0.5 \mathrm{M} \mathrm{KCl}$, and the soluble 
fraction contained, as the only antigens detectable, large amounts of both the precursor-1 and precursor-2 components. Uncloubtedly other protein species were present but eluded detection since the antiserum did not contain antibodies specific for them. After the yolk particles had been lysed, the residual solid matter was washed and also extracted in $0.5 \mathrm{M} \mathrm{KCl}$. The resultant supernatant, termed

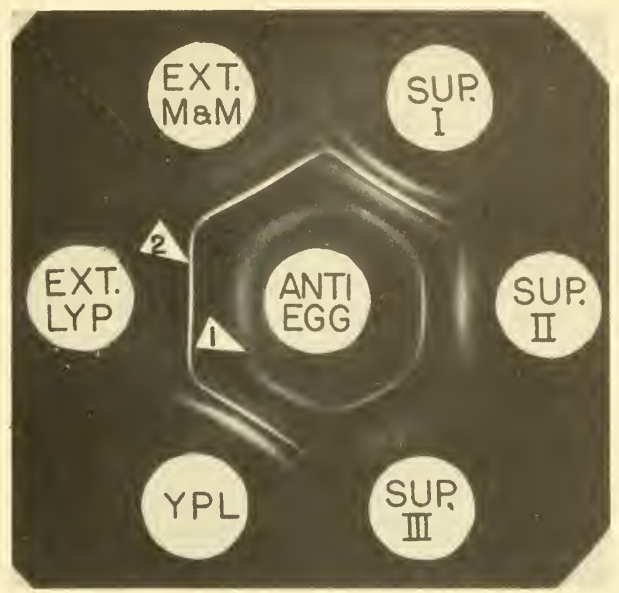

Fig. 3. Intracellular distribution of the precursor-1 and precursor-2 components. SUP. I, SUP. II, and SUP. III refer to the original supernatant, the yolk particles, and the two subsequent wash solutions, respectively. YPL represents the yolk-particle lysate, and EXT. LYP refers to the $0.5 \mathrm{M} \mathrm{KCl}$ extract of the structural material remaining after osmotic rupture of the yolk particles. EXT. M\&M indicates the $0.5 \mathrm{M} \mathrm{KCl}$ extract of the mitochondria-microsome fraction. Arrow 1 points to the precursor- 1 component band, and arrow 2 indicates the position of the precursor- 2 component band. It can be seen that these two components were the main antigens detected in the extracts of the mitochondria-microsome fraction and the lysed yolk-particle fraction. It would appear that the precursor-1 component is present as a "soluble" antigen within the yolk particles as well as in the surrounding cytoplasm.

the lysed yolk-particle extract, was immunochemically identical with the microsome-mitochondria extract. These relationships can be seen in Fig. 3. Both components of the mitotic apparatus have been found in all fractions studied from the unfertilized egg. However, two fractions, the lysed yolk-particle extract and the microsomemitochondria extract, yield solutions in which the precursor-1 com- 
ponent and the precursor-2 component were the only or major antigens detectable.

At this point, some speculation about the ubiquitous intracellular distribution of the precursor-1 component may be profitable. The much less studied precursor-2 component will be ignored, but not forgotten, in this discussion. There is little doubt that the precursor-1 component can exist in three "states." It is present in a "soluble" as well as in an insoluble form in the unfertilized egg, and is an important constituent of the mitotic apparatus. One can imagine a dynamic equilibrium to exist among the three "states" of the precursor-1 component. The insoluble fraction would represent the major pool of precursor-1 component, being converted into the soluble form as an intermediate step before its incorporation into the mitotic apparatus. An interesting consequence could be that the level of the "soluble" pool would remain unchanged during the formation of the mitotic apparatus and might or might not be greatly reduced when the mitotic apparatus is fully formed. Therefore, analysis of the "soluble" fraction for the precursor-1 component during development from the unfertilized egg to the two-celled stage would not reveal the fuctuations necessary to account for the amount incorporated into the mitotic apparatus.

Kane and Hersh (1959) describe some experiments that may have a bearing on the proposed interrelationships among the three "states" of the precursor-1 component. Ultracentrifugal studies on the soluble fraction of different stages from the unfertilized egg to the two-cell stage, in Arbacia, revealed a component whose concentration decreased with time and was undetectable at metaphase. It reappeared after cleavage. At the time, it was speculated (Mazia, 1957) that this behavior reflected the incorporation of soluble structural units into the developing mitotic apparatus. These analyses had been conducted on extracts obtained from ethanol-preserved material. When the experiment was repeated on S. purpuratus, no change in any component was detected that could be correlated with the buildup of the mitotic apparatus. Again, ethanol-preserved material had been used. For this reason, their experiments cannot be compared directly with the immunochemical data presented on the distribution of the precursor-1 component among the various fractions obtained by fractionation of living eggs in isotonic nonelectrolyte medium. However, their results are sufficiently consistent within themselves, so that the observed differences between 
the two species of sea urchin may be considered real. Quantitative measurements on various fractions from living material, cluring the time course from fertilization to cleavage, combined with parallel qualitative immunochemical determinations, could be expected to improve our understanding of the proposed interrelationships among the states of the precursor-1 component.

By way of summary, we can say that the unfertilized egg is endowed with the molecular subunits that have been identified as important structural elements in the mitotic apparatus. The formation of the mitotic apparatus may then be considered the result of the spatial rearrangement of the precursor- 1 and precursor- 2 components into the highly oriented aggregate, the mitotic apparatus. It has been further speculated that the main reservoir of these components in the unfertilized egg resides in existing subcellular moieties-the microsomes, mitochondria, and the structural elements of the yolk particles-and that the soluble fraction represents an intermediate form in the mobilization of the insoluble fraction into the mitotic apparatus.

Before we can fully accept the precursor concept for the origin of the mitotic apparatus in the sea urchin, it must be demonstrated that the precursor molecules that appear in the mitotic figure are indeed the same ones that occur in the unfertilized egg.

\section{References}

Anderson, N. G. 1956. Cell division Il: A theoretical approach to chromosomal movements and the division of the cell. Quart. Rev. Biol. 31: 243-269.

Boveri, T. 1887. Zellen-studien. G. Fischer, Jena.

Feinberg, J. G., and H. Grayson. 1959. A critical test of antigenic relationships. Nature 183: 987.

Flemming, W. 1882. Zellsubstanz, Kern und Zelltheilung. F. C. W. Vogel, Leipzig.

Gross, P. R. 1954. Alterations in the proteins of sea urchin homogenates treated with calcium. Biol. Bull. 107: 364-385.

Haurowitz, F. 1956. The nature of the protein molecule: Problems of protein structure. J. Cellular Comp. Physiol. Suppl. 1. 47: 1-16.

Hertwig, O. 1909. The Cell; Outlines of General Anatomy and Physiology. Sonnenschein \& Co., London.

Jensen, E. V. 1959. Sulfhydryl-disulfide interchange. Science 130. 1319-1323.

KaBAT, E. A. 1957. Size and heterogeneity of the combining sites on an antibody molecule. J. Cellular Comp. Physiol. Suppl. 1. 50: 79-102.

KANE, R. E., and R. T. Hersh. 1959. The isolation and preliminary characterization of a major soluble protein of the sea urchin egg. Exptl. Cell Research 16: 59-69.

Kawamura, N., and K. Dan. 1958. A cytochemical study of the sulfhydryl groups of sea urchin eggs during the first cleavage. J. Biophys. Biochem. Cytol. 4:615620.

Landsteiner, K. 1945. The Specificity of Serological Reactions. 2d rev. ed. Harvard University Press, Cambridge, Mass. 
Mazia, D. 1957. In W. D. McElroy and B. H. Glass (eds.). Symposium on Chem. Basis Heredity. Johns Hopkins Univ., McCollum-Pratt Inst. Contrib. No. 153.

Mazia, D. 1958. Cell division. Harvey Lectures Ser. 52 (1957-58): 130-170.

Mazia, D. 1959. In R. Benesch et al. (eds.). Sulfur in Proteins. Academic Press, Inc., New York.

MAZIA, D., and K. DAN. 1952. The isolation and biochemical characterization of the mitotic apparatus of dividing cells. Proc. Nat. Acad. Sci. U. S. 38: 826-838.

Mazia, D., and J. Roslansky, 1956. The quantitative relations between total cell proteins and the proteins of the mitotic apparatus. Protoplasma 46: 528-534.

MaZia, D., and A. M. Zimmerman. 1958. SH compounds in mitosis. I1. The effect of mercaptoethanol on the structure of the mitotic apparatus in sea urchin eggs. Exptl. Cell Research 15: 138-153.

NeufELd, E. F., and D. Mazia. 1957. Nonprotein sulfhydryl compounds in the division of eggs of Strongylocentrotus purpuratus. Exptl. Cell Research 13: 622-624.

Ouchterlony, O. 1949. Antigen-antibody reactions in gels. Acta Pathol. Microbiol. Scand. 26: 507-515.

Ouchterlony, O. 1958. Diffusion in gel methods for immunological analysis. Progress in Allergy 5: 1-78.

Oudin, J. 1946. Méthode d'analyse immuno-chimique par précipitation spécifique en milieu gélifié. Compt. rend. 222: 115-116.

Oudin, J. 1952. Specific precipitation in gels and its application to immunochemical analysis. Methods in Med. Research 5: 335-378.

Rapkine, L. 1931. Sur les processus chimiques au cours de la division cellulaire. Ann. physiol. physicochim. Biol. 7: 382-418.

SAKaI, H., and K. DAN. 1959. Studies on sulfhydryl groups during cell division of sea urchin eggs. Exptl. Cell Research 16: 24-41.

Shimamura, T., T. Ota, and T. Hishida, 1957. Cytochemical studies on the mitotic spindle. Symposia Soc. Cellular Chem. 6: 21-32.

Stern, H. 1956. Sulfhydryl groups and cell division. Science 124: 1292-1293.

STERN, H. 1958. Variations in sulfhydryl concentrations during microsporocyte meiosis in the anthers of Lilium and Trillium. J. Biophys. Biochem. Cytol. 4: 157161.

Strasburger, E. 1880. Zellbildung und Zelltheilung. G. Fischer, Jena.

Wilson, E. B. 1925. The Cell in Development and Heredity. 3d ed. The Macmillan Co., New York.

\section{DISCUSSION}

\section{V. Edds, Jr., H. A. Went, A. C. Giese}

DR. EDDs (Brown University): Would you care to speculate on the mechanism responsible for concentrating and ordering the precursor molecules in the spindle?

Dr. Went: There is evidence, as I have indicated, that the precursor molecules are rather widely distributed throughout the cytoplasm of the unfertilized egg, but we know nothing regarding the forces that align the precursor molecules into an orderly array. It is fairly clear that the centriole is, in some unknown manner, responsible for this orientation. Experiments with model systems (Anderson, 1956) show that one can get precipitation of proteins in solution radiating from a point which can be analogized to the centriole. Aggregation and alignment of macromolecules in model systems have been thought of in terms of surface charges and changes in their distribution or strength, conferring preferential aggregation patterns on the macromolecules. However, any resemblance between precipitation patterns observed in 
in vitro and in vivo systems may be only coincidence, with no fundamental mechanism in common. I don't feel justified in speculating further on what is responsible for bringing the precursor molecules sufficiently close to one another for short-range forces to bind them together into the definitive mitotic apparatus.

Dr. Giese (Stanford University): Has any study been made of the constituents of the centriole and their effect on spindle formation?

Dr. Went: No, not that I am aware of. However, this problem is now under investigation in Professor Mazia's laboratory. It is in its infancy, for a method still has to be perfected whereby centrioles, or their constituents, can be freed from undesired cytoplasmic matter. Then it is a question of whether or not enough material can be obtained, for the centriole is a very small entity. 



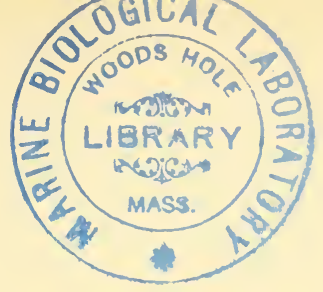

\title{
The Submicroscopic Basis of Bacterial Photosynthesis: The Chromatophore ${ }^{1}$
}

\author{
J. A. Bergeron ${ }^{2}$ and R. C. Fuller ${ }^{2}$
}

\section{Introduction}

The preceding symposium papers have considered submicroscopic aspects of objects with which biologists are already familiar at the microscopic or gross anatomical level, i.e., the collagenous fiber, the chloroplast, the mitotic spindle, and the myelin sheath. By contrast, the subject of this paper is less familiar because the bacterial chromatophore is itself submicroscopic and, as a consequence, was not identified until recently. Since the subject is new, it seems apropos to indicate the value of the bacterial chromatophore for studying photosynthesis.

Sixteen years ago, Ruben (1943) suggested that the energy of the pyrophosphate bond of adenosine triphosphate (ATP) could supply the energy needed for the photosynthetic assimilation of carbon dioxide. The following year, Emerson et al. (1944) proposed that the function of light energy in photosynthesis is the formation of energy-rich phosphate bonds. The concept of ATP as the fuel of photosynthesis gradually gained acceptance as the path of carbon during photosynthesis became clearer and as knowledge of carbohydrate phosphate interconversion improved. The search for a direct relation between light absorption and ATP formation culminated with the demonstration of photosynthetic phosphorylation by chloroplast preparations (Arnon et al., 1954a, 1954b) and by subcellular preparations of the non-sulfur purple bacterium, Rhodospirillum rubrum (Frenkel, 1954). Currently, the mechanism by

${ }^{1}$ Research carried out at Brookhaven National Laboratory under the auspices of the U.'S. Atomic Energy Commission. We are indebted to Mr. Mark Gettner, Mr. Walter Geisbusch, and Misses Gayle Schaeff, Catherine Kostuk, and Geraldine David for technical assistance.

${ }^{2}$ Biology Department, Brookhaven National Laboratory, Upton, New York. 
which light energy is used to create the energy-rich bond of the terminal phosphate of ATP is receiving considerable attention.

In higher plants, all of the many reactions of photosynthesis are integrated in a complex organelle, the chloroplast. The modern evidence in support of this old concept has been summarized by Arnon (1958). In order to study the energy-supplying step directly; it is desirable to eliminate all of the non-pertinent variables and use

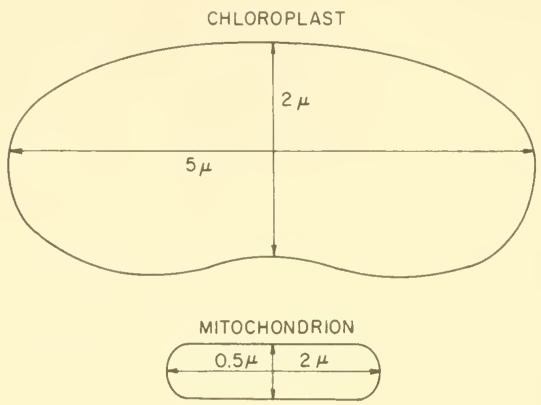

CHROMATOPHORE

$\cdot 0.03 \mu$

Fig. 1. Relative size of organelles. There are great differences in the level of organization of the organelles which supply chemical energy. The submicroscopic chromatophore converts light energy into the readily available chemical energy of the pyrophosphate bond of ATP. The mitochondrion, which lies just within the microscopic range, performs successive oxidations to generate ATP from substrates which act as energy reservoirs. The microscopic chloroplast converts light energy into pyrophosphate bond energy and uses the ATP to forge carbon dioxide and water into stable energy reserves.

the simplest possible system. The principal subject of this paper, the chromatophore of the purple sulfur bacterium 'Chromatium,' meets these conditions remarkably well since it is smaller than the chloroplast by two orders of magnitude (Fig. 1) and appears to have only one photosynthetic role, namely, the light-dependent formation of adenosine triphosphate (ATP) from adenosine diphosphate (ADP) and inorganic phosphate. 
The concept of the chromatophore as a photochemical organelle is beginning to assume definite form. Until recently it was believed that the pigments of photosynthetic bacteria are freely dispersed throughout the cell in the form of protein complexes. The chromatophore, in name at least, came into being in 1952, when Pardee, Schachman, and Stanier found that the photosynthetic pigments in extracts of $R$. rubrum sedimented much more rapidly than anticipated. The electron micrographs of shadowed specimens of these chromatophores showed thin disks about $1100 \AA$ in diameter. It was assumed that the disks were derived from spheres with an average diameter of about $600 \AA$. This value agreed roughly (Schachman et al., 1952) with the diameter $(400 \AA)$ calculated by Stokes' relation from the sedimentation constant $(200 S)$ of a purified preparation with a partial specific volume of 0.73 (assumed). The estimated molecular weight of these $R$. rubrum chromatophores was thirty million. At the same time, Thomas (1952) also published electron micrographs of shadowed specimens prepared from crude extracts of several photosynthetic bacteria. These preparations also contained thin disks. Reasoning by analogy with similar preparations from chloroplasts, Thomas described these objects as "lamellae" and as "grana," or stacks of lamellae. Despite the obvious differences in interpretation, these reports had in common the suggestion that the pigments of photosynthetic bacteria are localized in specific structures. Subsequently, when Frenkel (1954) described photophosphorylation by subcellular preparations of $R$. rubrum, it became logical to assume that the pigments of photosynthetic bacteria are integrated structurally and functionally at the submicroscopic level.

With this point of view, we began a systematic study of the structure and function of the photochemical apparatus of the purple sulfur bacterium, Chromatium strain D. Several properties of this organism made it especially attractive. Many photosynthetic organisms have the ability to form ATP by non-photosynthetic pathways and can grow aerobically in the dark. Chromatium is an obligate phototroph and an obligate anaerobe (van Niel, 19.31, 1935, 1936); thus, in this organism ATP formation seems to be completely lightdependent (see general discussion by van Niel, 1956). The synthetic' abilities are well developed, and Chromatium, given light, can live and grow anaerobically in simple salt solutions containing carbonate and hydrogen sulfide. 
The systematic study has progressively elevated the status of the chromatophore from that of a submicroscopic, pigmented particle which appears when the cells are broken, to the status of a photochemical organelle with a well-defined structure and a specific function. Using this point of view we shall consider the chromatophore at three conceptual levels: (1) as a pigmented particle, (2) as the structural and functional unit of photophosphorylation, and (3) as the photochemical organelle. Then we will present our interpretation of the electron-optical image of the chromatophore and a hypothesis of the molecular architecture.

\section{The Chromatophore as a Submicroscopic Pigmented Particle}

Isolation. The structures which we identify (Bergeron et al., 1957) as the chromatophores of Chromatium can be obtained in quantity by the procedure which is outlined in Fig. 2. The buffered $0.5 M$ sucrose medium is a recent refinement which was designed to maintain the physiological relationship between the carotenoid pigments and the bacteriochlorophyll (Bergeron, 1958; Bergeron and Fuller, 1959; Anderson et al., 1958). After the chromatophores have sedimented, the preparation is washed by resuspension and recen-

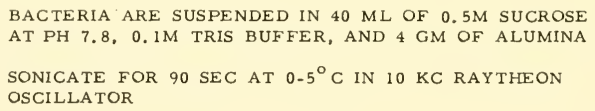

BACTERIA ARE SUSPENDED IN $40 \mathrm{ML}$ OF $0.5 \mathrm{M}$ SUCROSE AT PH 7.8, 0.IM TRIS BUFFER, AND 4 GM OF ALUMINA SONICATE FOR $90 \mathrm{SEC} \mathrm{AT} 0-5^{\circ} \mathrm{C}$ IN $10 \mathrm{KC}$ RAYTHEON OSCILLATOR

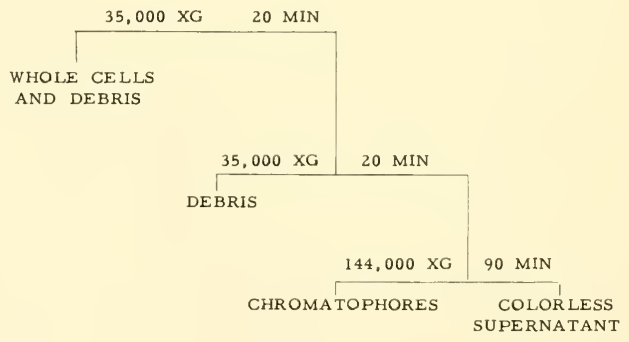

Fig. 2. The procedure used for isolating the Chromatium chromatophores. The final preparation is washed two or three times by recentrifugation in the buffered medium. 
trifugation for 90 minutes at $144,000 \mathrm{~g}$ in the same medium. For some purposes the chromatophores can be washed by repeated centrifugation in dilute buffer or distilled water. The isolated chromatophores can be lyophilized and stored over phosphorus pentoxide at reduced temperature $\left(-20^{\circ} \mathrm{C}\right)$ for long periods without significant loss in enzymic activity or alteration in the over-all physicochemical properties.

Physicochemical Properties. About 90 per cent of the cellular pigments are recovered in the final fraction. The absorption spectrum between $350 \mathrm{~m} \mu$ and $1000 \mathrm{~m} \mu$ of the chromatophores prepared in the sucrose medium corresponds with the absorption spectrum of the original cells (Fig. 3). It might be expected, a priori, that the chromatophores are rather fragile, but the reverse is true. Prolonged oscillation at 10 kilocycles in dilute buffer does not produce appreciable fragmentation. The chromatophore is hydrophilic and has an isoelectric point between $\mathrm{pH} 3$ and 4; this is in accordance with

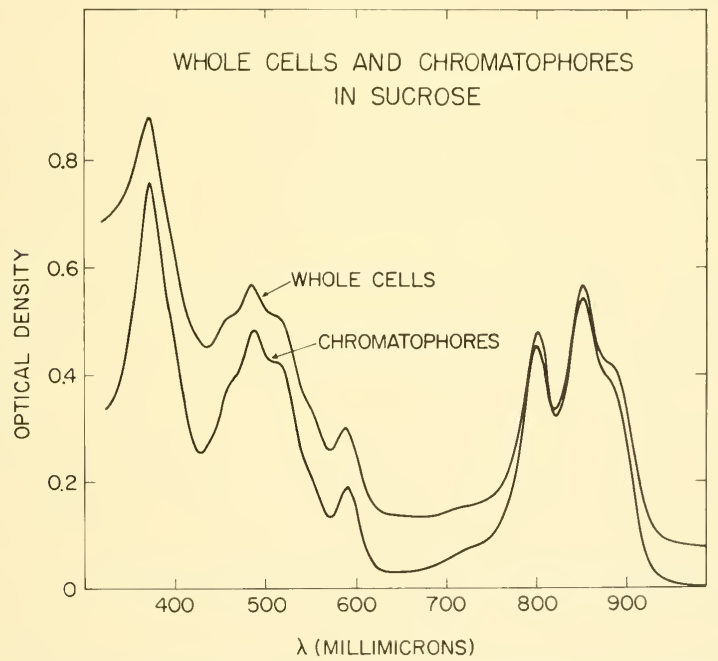

Fig. 3. A spectrophotometric comparison of chromatophores, isolated in the buffered $0.5 \mathrm{M}$ sucrose medium, with the intact organisms, suspended in Hendley's medium. The spectra were measured (Cary Model 14) through opal glass to reduce the divergence caused by light-scattering at the lower wave lengths. The close correspondence indicates that the photosynthetic pigments are not disturbed by this isolation procedure. 
the observations on the minimum solubility and charge reversal of crude extracts (Katz and Wassink, 1939; French, 1940). The pronounced color of the chromatophores facilitates their identification in the analytical ultracentrifuge. The color is related to only one peak; thus the chromatophore preparation is monodisperse. The sedimentation constant, measured at extreme dilution in de-ionized water at neutrality, is $143 \mathrm{~S}$ corrected to $20^{\circ} \mathrm{C}$. Since the chromatophore is not disrupted by lyophilization, it is possible to measure the density of aqueous suspensions in terms of the gram fraction. The

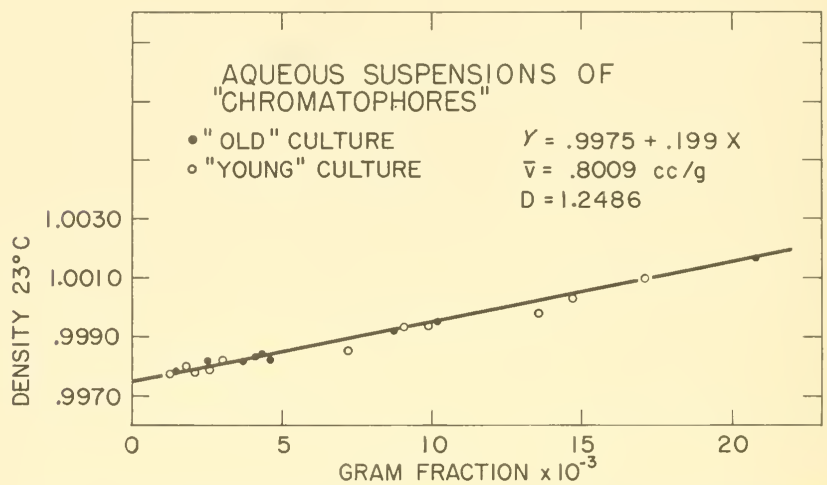

Fig. 4. The relation between the gram fraction and the density of aqueous suspensions of lyophilized chromatophores. The equation of the line, $Y=0.9975$ $+0.199 x$, can be used to calculate the partial specific volume $(\bar{v})$ and density (D) of the chromatophore.

resulting plot (Fig. 4) is linear and can be used to calculate the partial specific volume $(0.8 \mathrm{cc} / \mathrm{g})$ at extreme dilution in pure water. Since the chromatophores are spherical (see below), Stokes' relation is applicable and an average particle diameter of $320 \AA$ is obtained. The molecular weight is about thirteen million.

Thus the chromatophore preparation contains the photosynthetic pigments in a physiological relationship and is composed of a rather uniform population of submicroscopic particles.

Composition. The chemical composition of the chromatophore is known reasonably well; about 89 per cent of the dry weight is 
present in components which are not free to exchange. These "fixed" components-protein, phospholipid, bacteriochlorophyll, and the carotenoids-are integral parts of the structure. Our data (Table 1) for the chromatophore (Bergeron, 1958), if converted to a milligram protein basis, agree very well with the data of Newton and Newton (1957) for the fraction which they called the "small particles" (chromatophores, see below). Since these authors reported that the

TABLE 1

\section{Chromatophore Composition}

\begin{tabular}{lccclcc}
\hline \multicolumn{1}{c}{ Substance } & $\begin{array}{c}\text { Per } \\
\text { Cent }\end{array}$ & G/“Mole” Ch & $\begin{array}{c}\text { Molecular } \\
\text { Weight }\end{array}$ & $\begin{array}{c}\text { Mole- } \\
\text { cules Ch }\end{array}$ & $\begin{array}{c}\text { Mole } \\
\text { Ratio }\end{array}$ \\
\hline Carotenoid $\ldots \ldots \ldots \ldots$ & 1.5 & $2.0 \times 10^{5}$ & 700 & 300 & 1 \\
Bacteriochlorophyll $\ldots$ & 4.2 & $5.5 \times 10^{5}$ & 900 & 600 & 2 \\
Phospholipid $\ldots \ldots \ldots$ & 22.3 & $30 \times 10^{5}$ & $900^{\circ}$ & 3000 & 10 \\
Protein $\ldots \ldots \ldots \ldots \ldots$ & 61.0 & $80 \times 10^{5}$ & $120+/$ A.A. & 67,000 A.A. & 220 A.A. \\
DNA $\ldots \ldots \ldots \ldots \ldots$ & 0 & - & - & - & - \\
PNA $\ldots \ldots \ldots \ldots \ldots$ & 0 & - & - & - & - \\
Other $\ldots \ldots \ldots \ldots \ldots$ & 11.0 & $12.5 \times 10^{5}$ & - & - & - \\
\hline
\end{tabular}

The per cent composition on a dry-weight basis has been converted to grams per "mole" Ch by employing the "molecular weight" of thirteen million. By assigning an average molecular weight to each substance, one can obtain an estimate of the number of molecules of each substance in a chromatophore. The mole ratio represents a "minimal unit" of composition. This unit represents a molecular weight of about 40,000 .

- Cephalin with $\mathrm{C}_{16}$ fatty aeids.

$\uparrow$ Round value for average amino acid residue.

phospholipid contains ethanolamine, phosphorus, and glycerol in a 1:1:1 ratio, we have treated the phospholipid as a cephalin. The "molecular weight" of the chromatophore has been employed to calculate the amount of each "fixed" substance in a "mole" of chromatophores. By assigning an average molecular weight to each substance, one can estimate the number of molecules of each type in a chromatophore. By this method of calculation, the chromatophore contains 300 carotenoid molecules, 600 chlorophyll molecules, 3000 cephalin molecules, and protein equivalent to 67,000 amino acids. The hypothetical "minimal unit of composition" (empirical formula) is 1 carotenoid molecule, 2 bacteriochlorophyll molecules, 10 phospholipid molecules, and protein equivalent to 220 amino acids. The "formula weight" is about 40,000 . 
It should be noted that the carotenoids occur as a variable mixture of several types, particularly lycopene, spirilloxanthin, and lycoxanthin (Goodwin and Land, 1956). It is also possible to interfere with carotenoid synthesis by treatment with diphenylamine (Goodwin and Osman, 1954) and obtain chromatophores which appear to totally lack the colored carotenoids (Anderson and Fuller, 1958; Bergeron and Fuller, 1959); however, poorly colored precursors appear to be present instead (reviewed by Stanier, 1958). Since the carotenoid:bacteriochlorophyll ratio observed above agrees with the ratio reported by Newton and Newton (1957), and with the maximum value observed by Stanier (1958) in Rhodopseudomonas spheroides, it seems safe to assume that these chromatophores also contained about the maximum amount of colored carotenoids.

The status of polysaccharide as a "fixed" component is debatable. Newton and Newton (1957) describe polysaccharide in their "small particle" fraction $(100,000 \mathrm{~g}, 90 \mathrm{~min})$. Their data show that in passing from the "chromatophore fraction" $(25,000 \mathrm{~g}, 60 \mathrm{~min})$ to the "small particle," the phospholipid and chlorophyll content double on a protein basis while the polysaccharide content decreases by a factor of three and one-half. These authors suggested that the chromatophore consists of pigmented macromolecules in a carbohydrate envelope. Newton (1958) has elaborated this point of view on the basis of the immunochemical properties of intact cells and pigmented fractions.

Our data, particularly the electron microscope observations on thin sections ( see below), indicate that the chromatophore $(144,000$ $\mathrm{g}, 90 \mathrm{~min})$ is in the "small particle" fraction of Newton $(100,000 \mathrm{~g}$, $60 \mathrm{~min}$ ). Irregular cell fragments are common in the $25,000 \mathrm{~g}$ fraction, which was identified with the chromatophore by Newton. For this reason, we interpret the data on carbohydrate release as evidence that it is a contaminant and not an integral part of the chromatophore.

The relation to the chromatophores of several biologically important compounds, i.e., pyridine nucleotides, cytochrome, coenzyme $Q_{\bar{\tau}}$, and vitamin $\mathrm{K}$, is of special interest but remains obscure.

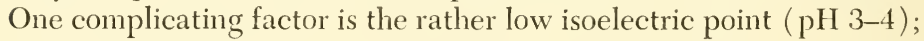
as a consequence, the chromatophore has considerable net charge at neutrality. This appears to be a factor in explaining a net uptake by chromatophores of added cytochrome c (Newton and Newton, 1957 ). 


\section{The Chromatophore as the Structural and Functional Unit of Photophosphorylation}

Experiments with crude extracts of Chromatium by Williams (1956) suggested that photophosphorylation might have occurred as in the R. rubrum preparations used by Frenkel (1954). Experiments with the purified chromatophore fraction (Fuller and Anderson, 1957, 1958) have unequivocally demonstrated the light-dependent formation of ATP from ADP and inorganic phosphate. The

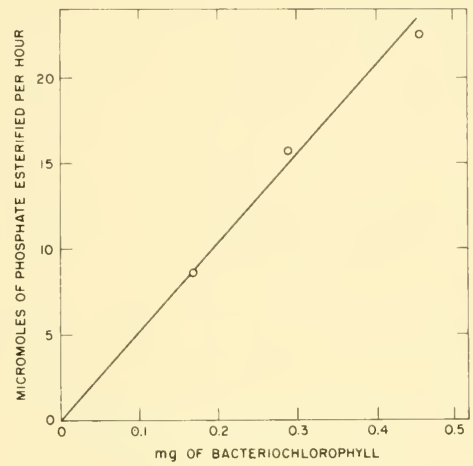

Fig. 5. A plot of the rate of ATP formation against an index of chromatophore concentration. The proportionality which results indicates that photophosphorylation does not depend upon interaction between chromatophores.

reaction also requires $\mathrm{Mg}^{++}$and is stimulated by small amounts of the uncolored supernatant fluid. Two distinct factors are involved: one is heat-labile, non-dialyzable, and can be precipitated by $35-45$ per cent ammonium sulfate; the other is heat-stable, dialyzable, and can be replaced by catalytic amounts of succinate or reduced diphosphopyridine nucleotide (DPNH). Photophosphorylation by Chromatium fractions has also been studied by Newton and Kamen (1957).

The rate of photophosphorylation by the purified chromatophores is a linear function (Fig. 5) of the chromatophore concentration (indirectly expressed by the bacteriochlorophyll content). A linear dependence indicates that the chromatophores are not random fragments of a photophosphorylating system; otherwise a falling rate 
would be expected with progressive dilution. Since the pigment content of the chromatophore is known, it can be calculated that, in these experiments, each chromatophore was forming about 500 highenergy phosphate bonds per minute (cf. Bergeron, 1958).

The enzymes involved in the assimilation of carbon dioxide are not integrated in the chromatophore and are found in the uncolored supernatant fluid. If the chromatophores are recombined with the supernatant fluid, the reconstituted system fixes $\mathrm{CO}_{2}$ when the system is illuminated (Table 2). When the light:dark ratios of $\mathrm{CO}$. incorporation are compared, the reconstituted system is 30 per cent as effective as the intact cells. It should be noted in passing that, unlike higher plants, Chromatium does not synthesize large quantities of reserve carbohydrates, and the rate of incorporation of $\mathrm{CO}_{2}$ into amino acids such as aspartic is proportionately more rapid.

TABLE 2

$\mathrm{CO}_{2}$-Fixation by Various Cell Fractions of Chromatium. $1 \mathrm{Hr}$ *

\begin{tabular}{|c|c|c|}
\hline Cell Fraction & $\begin{array}{l}\text { Dark } \\
\text { or } \\
\text { Light }\end{array}$ & $\begin{array}{c}\text { Total } \mathrm{CO}_{2} \text { Fixed } \\
\text { as } \mathrm{C}^{14} \text { in Counts/Min }\end{array}$ \\
\hline Whole cells $\ldots \ldots \ldots \ldots \ldots \ldots \ldots \ldots \ldots$ & Light & $4,500,000$ \\
\hline Whole cells $\ldots \ldots \ldots \ldots \ldots \ldots \ldots \ldots \ldots \ldots \ldots$ & Dark & 50,000 \\
\hline 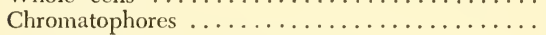 & Light & 750 \\
\hline Supernatant $\ldots \ldots \ldots \ldots \ldots \ldots \ldots \ldots \ldots \ldots$ & Light & 1,750 \\
\hline Supernatant and chromatophores $\ldots \ldots \ldots \ldots \ldots$ & Dark & 1,950 \\
\hline Supernatant and chromatophores $\ldots \ldots \ldots \ldots \ldots$ & Light & 69,000 \\
\hline
\end{tabular}

- The cell-fractionation procedure separates the energy-yielding and energy-requiring steps of photosynthesis. The chromatophore fraction catalyzes photophosphorylation, but the enzymes which use ATP for the synthetic reactions are in the supernatant fraction. When the two fractions are combined, their fixation of $\mathrm{CO}_{2}$ proceeds in the light.

\section{The Chromatophore as the Photochemical Organelle}

The procedure used to isolate a given set of cellular properties serves as an operational definition of the fraction; correlated data from both direct and indirect methods of observation are needed to characterize the fraction and relate it to the cells from which it is obtained. In this investigation, the requirement for direct observation was met by the electron microscope study of intact cells, crude pigmented fractions, and the purified chromatophore fraction (Ber- 
geron et al., 1957; Bergeron, 1958). The most profitable observations were made with thin sections of methacrylate-embedded pellets of preparations which had been fixed for 1 hour at $25^{\circ} \mathrm{C}$ in 1 per cent osmium tetroxide solutions. The fixatives were buffered at $\mathrm{pH} 7.4$ with Veronal-HCl and matched as closely as possible in ion balance and osmolarity with the culture or suspension medium. All substances which reacted with the osmium tetroxide were excluded.

In such sections (Fig. 6), the bacteria have smooth contours and are bounded by two distinct membranes, the cell wall and the cytoplasmic membrane. The obvious and predominant intracellular structures are the chromatophores. This abundance, though puzzling, agrees with the indirect measurements; it was calculated, by comparing the bacteriochlorophyll content of lyophilized cells and of lyophilized chromatophores, that the chromatophores represent about two-thirds of the total cellular solids. Sometimes the arrangement of chromatophores is so regular that it suggests a crystalline pattern; consequently, the details of the chromatophore are observed more easily in thin sections of the chromatophore fraction (Fig. 7).

The chromatophores occur as individuals, clumps, and occasionally as chains. These configurations can be regarded as secondary associations produced during fixation since the fixatives cause the progressive flocculation and precipitation of dilute suspensions of either the cells or the chromatophores. There is no evidence that the chromatophore is a fragment of a more highly organized, larger organelle.

The sections show that even the finest preparations are not perfectly clean. They contain a small number of larger vesicular structures which resemble the chromatophore; in addition, small particles $(\sim 100 \AA)$ of high intrinsic electron density are present in variable numbers. It is interesting to note that these impurities were not noticed in the analytical ultracentrifuge. This illustrates the sensitivity achieved by using thin sections of sedimented fractions as an assay of homogeneity.

In studying the crude cell fractions we were surprised to find large cell fragments in which the contents appeared to be quite undisturbed. We assume that the cytoplasm has "gelled" as a response to stimuli received during the sonic oscillation at 10 kilocycles in the cold $\left(0-6^{\circ} \mathrm{C}\right)$, or because of the formation of nucleic acid gels during rupture. One consequence of this phenomenon is 


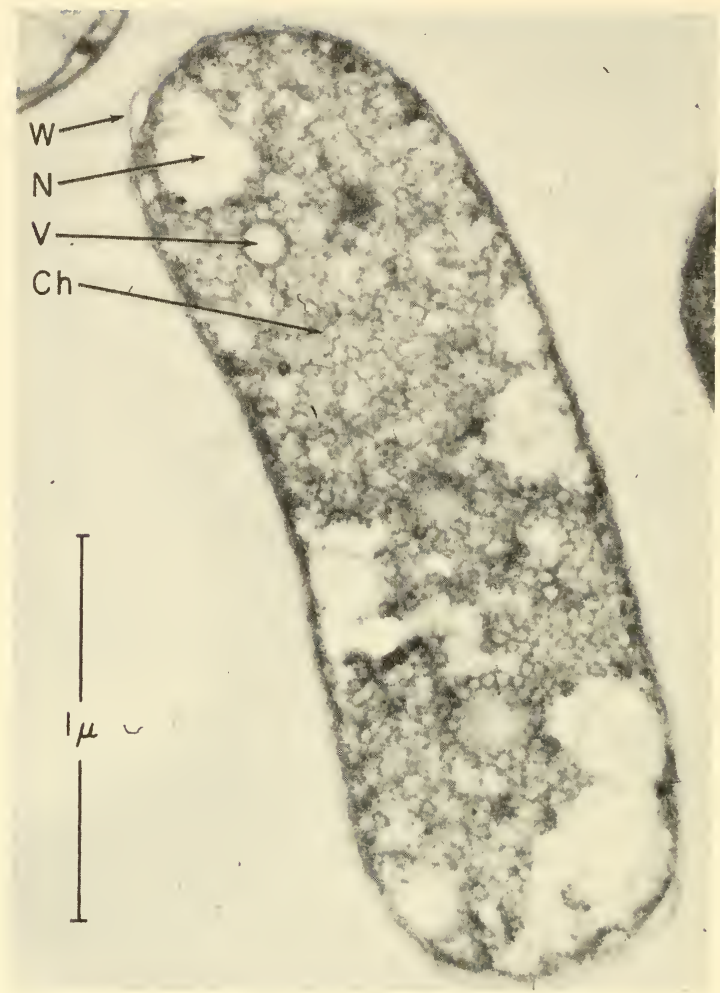

Fig. 6. An electron micrograph of a thin section of the purple sulfur bacterium, Chromatium. The organism is smooth-contoured and is bounded by a dual membrane which can separate into two distinct structures, the cell wall (W) and the plasma membrane. The cell is filled with the chromatophores (Ch), which are minute vesicles. These appear as annular images with an outer diameter of about $300 \AA$ and a cortical thickness of about $70 \AA$. Large vesicles (V), about $1000 \AA$ in diameter, are also visible. The irregular areas of low density $(\mathrm{N})$ are considered to be parts of an irregularly shaped nuclear compartment. The closely packed chromatophores conceal the small particles which are observed in the fractions. $\times 48,000$.

that the chromatophores literally have to be shaken out of the broken cells. The different rates of sedimentation of the pigmented cell fragments and of the individual chromatophores led us earlier to the artificial and erroneous distinction between "large" and 


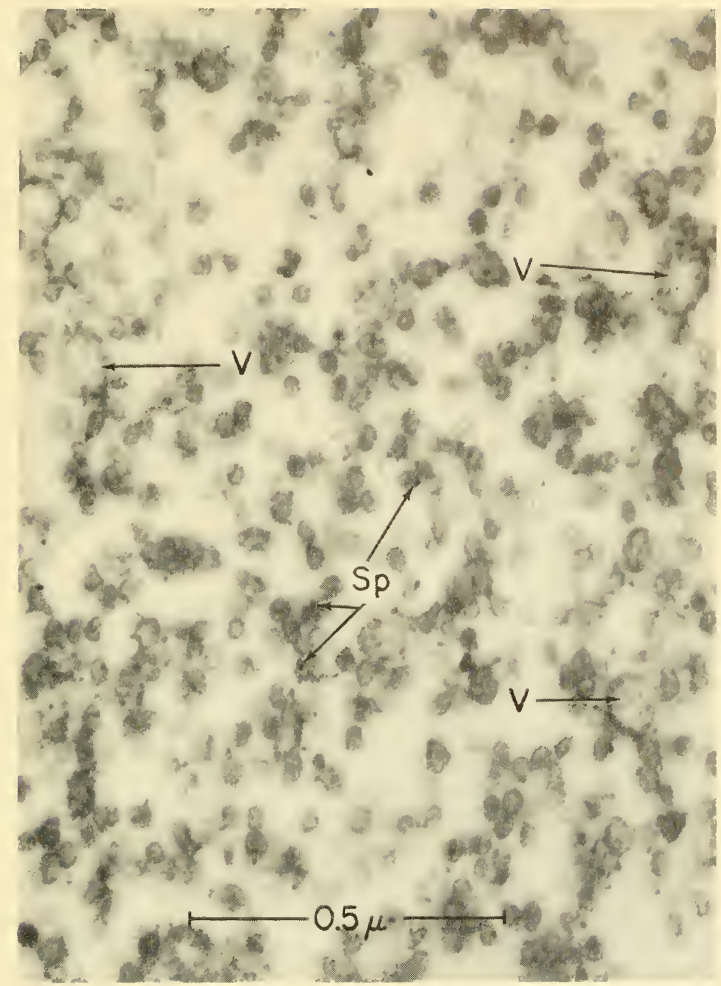

Fig. 7. An electron micrograph of a thin section of a typical chromatophore preparation. The chromatophores occur singly, in clumps, and occasionally as chains. This aggregation seems to be produced by the fixative. Larger vesicles (V) may be present. It is not known whether or not these structures are large chromatophores. Small dense particles (Sp) are often present as contaminants. $\times 78,000$.

"small" chromatophores, just as Newton and Newton (1957) have distinguished "chromatophores" and "small particles."

We have already reviewed the biochemical reasons for doubting the idea (Newton and Newton, 1957) that the structure which we identify as the chromatophore is a fragment of the "real" chromatophore. When we consider, in addition, the electron microscopic observations on intracellular components and remember that two- 
thirds of the cellular solids are chromatophores, it is clear that, although chromatophores are present at the surface of the cytoplasm, the structure with which they are associated is the cell itself.

In our opinion, the data obtained in this systematic study are consistent with the view that the chromatophore is the photochemical organelle of Chromatium (Bergeron, 1958). Since the electron micrographs of thin sections of several other photosynthetic bacteria, Rhodospirillum rubrum, Rhodopseudomonas spheroides, and Chlorobium limicola, also show vesicular inclusions in abundance (Vatter and Wolfe, 1958), it is possible that this view has some general validity. Since the vesicles in the non-sulfur photosynthetic bacteria are much larger than the Chromatium chromatophore, they may be more complicated. For example, the Rhodospirillum rubrum chromatophore, which has been studied extensively, though not as systematically, appears to be more complex (see review by Frenkel, 1959). It is also possible, considering the variety of these microorganisms, that lamellated pigmented structures exist. The electron micrographs of Rhodomicrobium vannielii (Vatter et al., 1959) suggest this possibility. It remains to be established, however, that these lamellae are pigmented. It is also conceivable that in some photosynthetic bacteria the pigments will not be integrated into vesicular structures which can perform the complete sequence of events involved in photophosphorylation. ${ }^{3}$

\section{Interpretation of the Electron-Optical Image}

Both the physicochemical and the electron microscope data indicate that the isolated chromatophore is a sphere. The electron microscope observations on the thin sections of the organism also reveal discrete spherical structures; anastomoses or tubular profiles have not been observed. The annular form of the image of the sectioned chromatophore shows that the central region does not scatter 50kilovolt electrons as effectively as does the cortical region. Since the chromatophores are frequently seen in direct contact, we can infer that, within the limits of resolution, the outer boundary of the image

${ }^{3}$ The suggestion that photosynthesis can proceed at an even simpler level of structural organization than the chromatophore has been supported by study of the green sulfur bacterium, Chlorobium thiosulfatophilum. In this instance, the photosynthetic pigments are recovered in a macromolecule with a molecular weight of about 1 million (J. A. Bergeron and R. C. Fuller in "Biological Structure and Function,” IUB/IUBS Symposium, 1960, Academic Press, Inc., in press ). 
coincides with the surface. The average diameter observed in the thin sections of the chromatophores is $300 \AA$, which agrees very well with the value $(320 \AA)$ calculated by Stokes' relation from the sedimentation constant and density. We employ the latter as the diameter of the unfixed chromatophore. This agreement between the different measurements suggests that shrinkage has been minimized in the specimens used for electron microscopy.

The objective lens of the electron microscope has considerable depth of focus, and points which are separated by considerable distances in the direction of the axis of the lens can be superimposed in the final image. Since the chromatophores tend to pack very closely in the cell, image-overlap due to the depth of focus will obscure the chromatophores and produce optical artifacts if the sections contain more than one layer of chromatophores. This effect also makes it difficult to assign a value to the thickness of the cortex of the chromatophore. There are at least four intrinsic factors which influence the apparent thickness of the cortex in a section: the actual thickness of the cortex, the efficiency with which the electrons are scattered by this region, the thickness of the section, and the diameter of the chromatophore. The apparent thickness of the cortex in the thinnest sections is about $70 \AA$, but the actual thickness of this region must be less than this value. The magnitude of the deviation becomes clear (Fig. 8) when we compare the effect on the image of varying the thickness of sections through the center of a sphere $300 \AA$ in diameter with a completely opaque cortex. An apparent cortical thickness of $70 \AA$ can be obtained for actual values ranging from 25 to $60 \AA$ as the section thickness decreases from 200 to $100 \AA$. Since the cortex of the chromatophore is only moderately opaque to 50 kilovolt electrons and the thickness of the sections appears to be less than $200 \AA$, a cortical thickness of about $60 \AA$ seems reasonable.

Since the intrinsic electron density of most cellular constituents is rather low, procedures which enhance electron density are normally employed for electron microscopy. Practice has established osmium tetroxide, particularly in the buffered solution advocated by Palade (1952), as the standard or reference fixative. A classical aphorism, namely, that osmium tetroxide fixes and blackens fats, has been responsible for a tendency to assume that the patterns of electron density produced by this reagent reflect the deposition of the metal or its oxides in the lipid constituents of cellular structures. Nevertheless, the data have often suggested that protein rather than 
lipid is involved in defining the image of cytoplasmic membranes and lamellae (see review by Sjöstrand, 1959).

It is clear that the chromatophore is not a rigid structure, for the electron micrographs of shadowed preparations show that the chromatophore becomes a thin disk when it is dried upon a support. By the same token, water accounts for a considerable fraction of the

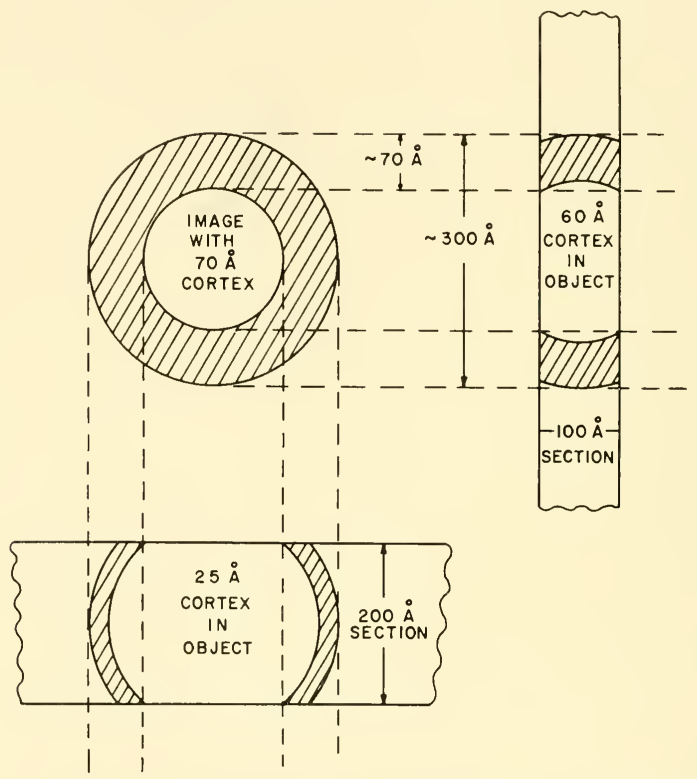

Fig. 8. A simple illustration of several factors which determine the image of the sectioned chromatophore. If the cortex were opaque, the effect of geometry and depth of focus could produce an apparent cortical thickness of $70 \AA$ for actual values between 25 and $60 \AA$ as the section thickness varied between 200 and $100 \AA$.

volume of the organelle. Since the chromatophore behaves and "looks" like a vesicle, it is probable that most of the structural materials are concentrated in a cortical region.

The properties of the chromatophore indicate that protein rather than lipid is present at the surface; for example, the chromatophore is hydrophilic, has an isoelectric point, and acts as an enzyme. In addition, the distortion or denaturation of protein is a prerequisite 
for the release of the lipids. Since the carotenoids, upon extraction, are accompanied by a proportionate amount of the phospholipid (Newton and Newton, 1957), these pigments can serve as indicators of the leaching of lipids in the solvents used in preparing specimens for electron microscopy. Using such indications, we observed that the image of the chromatophore was not appreciably different if obvious leaching had occurred or if leaching had been minimized by using the solvents at the lowest practical temperature and polymerizing the monomeric methacrylate at dry ice temperature.

It is a common practice to fix tissues rather briefly because exposure beyond an ill-defined optimum period in the customary buffered osmium tetroxide fixatives produces a pronounced deterioration of structure. In view of this practical limitation on fixation time, the loss of lipid could have been due to inadequate osmication or to an inherent lack of reactivity. These possibilities were tested by experiments (1) with lyophilized chromatophores, (2) with the proteinaceous residue obtained by extracting the lipids in a sequence of boiling solvents (ethanol, 24 hours; ether, 24 hours) in a Soxhlet apparatus, and (3) with the lipid films produced by evaporation of the combined extracts. The rate and extent of osmication of each of the three systems was determined by the change in dry weight as a function of time. Numerous samples containing about $10 \mathrm{mg}$ of material were exposed to the fixative in a pre-equilibrated moist chamber which contained a large volume of 1 per cent osmium tetroxide. At intervals, samples were removed and reweighed after dessication to constant weight.

The lipid films incorporated osmium rapidly and became black; in 1 hour the weight increased by 40 per cent. At this point, the bulk of the film was insolubilized, but about 10 per cent of the mass could be extracted by ether or methacrylate monomer; 20 per cent could be removed by ethanol. The incorporation of osmium was finished in 8 hours with a net weight increase of 50 per cent. If we use 900 as the average molecular weight of the lipids and assume that the osmium tetroxide is reduced to the metal (at. wt. 190.8), then at saturation each lipid molecule is related to two or three atoms of osmium.

Under comparable conditions, the defatted protein actually incorporated osmium more rapidly than the lipid films, but the net uptake was much less. The weight increment was 18 per cent in 1 hour. The final value was 20 per cent. The osmium content at 


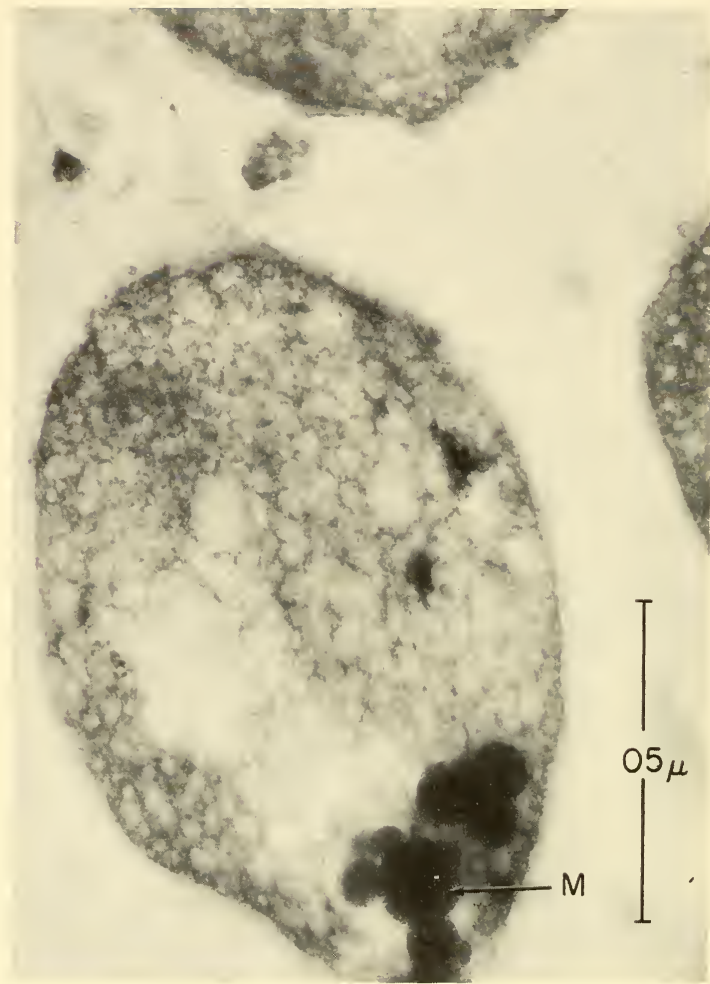

Fig. 9. An electron micrograph of a thin section of organisms which had been lyophilized and extracted in boiling solvents before fixation. The organisms were distorted by this procedure, but the bulk of the electron-dense material is still observed despite the absence of lipids. The metaphosphate inclusions (M) also persist. $\times 80,000$.

saturation represents a ratio of about one osmium atom for every eight amino acid residues.

The total uptake of osmium by the lyophilized chromatophores produced a 30 per cent increase in weight. This is essentially a summation of the uptake expected for the sum of the two fractions; however, the course of the reaction was not a simple summation of concomitant reactions by the protein and the lipid. In 1 hour the chromatophores had increased in weight by 15 per cent, or half of 
the final value; nevertheless, 70 per cent of the lipid could be extracted in ethanol. The incorporation of osmium continued gradually and required 3 days for completion. These data show that the rate of osmication of the lipids is greatly reduced when the lipids are integrated in the chromatophore. If, as we propose, the lipids are coated with protein ( see below), then it might be expected that osmication of the lipid would not proceed readily until the protein had been saturated. Be that as it may, it is reasonably clear that in our usual preparations the lipid does not contain much osmium and will not have a high electron densitr:

The role of osmicated protein in image formation is shown unequivocally by electron micrographs of lyophilized bacteria from which the lipids were removed by Soxhlet extraction before fixation. The organisms are distorted by this violent treatment, but the bulk of the electron-dense material is still present (Fig. 9). Taken collectively, these observations support the view that osmicated protein is imaged as the cortex of the chromatophore in our preparations.

It should be noted, however, that even if the lipid were totally osmicated it might not be revealed. Stoeckenius (1959) has studied thin sections of myelin forms prepared from phospholipid and fixed in osmium tetroxide. The periodicity of the lamellae agrees with the $40 \AA$-spacing expected for bimolecular leaflets, but the dense line is only 18-20 $\AA$ thick. If the phospholipids in the chromatophore are arranged in a monolayer (see below), the electron-dense line might be only $10 \AA$ in diameter, which is well within the limits of our measurement errors.

\section{Hypothesis of the Ultrastructure of the Chromatophore}

All of the protein can be accommodated in the region which is imaged as the cortex of the chromatophore. The volume of this zone $\left[\frac{4 \pi}{3}\left(160 \AA^{3}-100 \AA^{3}\right)\right]$ is $\left(1.3 \times 10^{7}\right) \AA^{3}$. There are about $1.3 \times 10^{-17}$ grams of protein or, alternatively; some 67,000 average amino acid residues (see above). The volume required by the protein is about $10^{7} \AA^{3}$ when typical (cf. Frey-Wyssling, 1953) values are chosen for either the partial specific volume $(0.78 \mathrm{cc} \mathrm{g})$ or the volume $\left(161 \mathrm{~A}^{3}\right)$ per residue. In view of the agreement of such volume considerations with the physicochemical and electron microscope data, it is reasonable to infer that the bulk of the protein is 
actually distributed in a layer about $60 \AA$ thick at the surface of the chromatophore.

It is generally assumed that the photosynthetic pigments exist in a condensed state, such as a monolayer, in vivo. This concept has been inferred repeatedly (see Rabinowitch, 1945, 1951, 1956) from considerations of the absorption spectrum, fluorescence, energy transfer, etc. In Chromatium, energy is transferred from the carotenoids to bacteriochlorophyll with an efficiency of 30 to 40 per cent (Duysens, 1952); nevertheless, these accessory pigments are not necessary as functional or structural components (Fig. 10) of the chromatophore. In addition, there is supposed to be a fundamental difference between the relationships of the two types of pigment with the protein. The bacteriochlorophyll is a clavate molecule; that is, the phytol "tail" is hydrophobic, but the porphyrin "head" is hydrophilic and can enter into intimate relation with protein. The carotenoids are pure lipids and cannot form strong bonds with protein.

The pigments of the chromatophore will fit in a monolayer about $25 \AA$ thick at the internal face of the proposed protein layer. The area available at this interface $\left[4 \pi(100 \AA)^{2}\right]$ is $\left(1.26 \times 10^{5}\right) \AA^{2}$. The carotenoids of purple bacteria are aliphatic $\mathrm{C}_{40}$ compounds about $25 \AA$ in length. Since the van der Waals radius of the methyl group is $2 \AA$, each of the 300 carotenoid molecules will require about $13 \AA^{2}$ at the interface, for a total of $4000 \AA^{2}$. The remaining area, (1.22 $\left.\times 10^{5}\right) \AA^{2}$, allows $200 \AA^{2}$ for each of the 600 molecules of bacteriochlorophyll. This value falls between the area of the porphyrin itself $\left(242 \AA^{2}\right)$ and the area occupied by the "head" of the chlorophyll molecule in the tilted position assumed in monolayers (Trurnit and Colmano, 1959). Similar calculations indicate that the 3000 cephalin molecules can be ordered with the polar groups directed into the central aqueous phase and with the non-polar fatty acid moieties forming a lipid phase in conjunction with the carotenoids and the phytol "tails." This asymmetric organization, though speculative, is consistent with the known physicochemical properties, the composition data, and the electron microscope observations.

The de novo origin of bacterial chromatophores seems to have been established by observations on Rhodospirillum rubrum (Pardee et al., 1952; Vatter and Wolfe, 1958). Such observations suggest that the chromatophores are assembled from smaller particles. In terms of the "fixed" components, the simplest representative build- 
ing block contains 1 carotenoid molecule, 2 bacteriochlorophyll molecules, 10 cephalin molecules, and protein containing 220 amino acids. Although a structural subunit with a molecular weight of

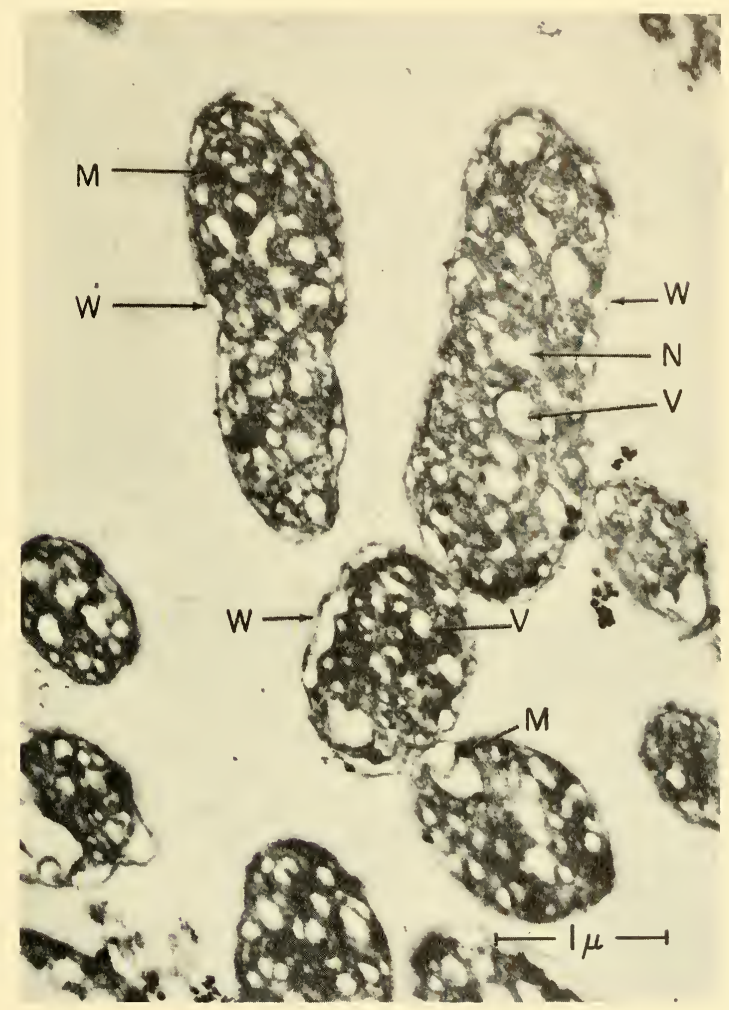

Fig. 10. An electron micrograph of thin sections of organisms in which the development of colored carotenoids has been prevented by diphenylamine treatment. The absence of these pigments does not alter the characteristic appearance of the chromatophores which are dispersed throughout the cytoplasm. M: Metaphosphate inclusions; N, V, W: see Fig. 6. $\times 22,000$.

about 40,000 seems ridiculously small, this agrees with the molecular weight of the chloroplastin obtained by digitonin extraction of Euglena chloroplasts (Wolken, 1956; Wolken and Schwertz, 1956). 
The area available at the surface $(R=160 \AA)$ for the protein of any subunit is about twice as large as the area available at the lipidprotein interface $(R=100 \AA)$. In the case of the hypothetical minimal unit, the area available at the lipid interface is only $420 \AA^{\circ}$. The protein could be folded, with the terminal groups brought to

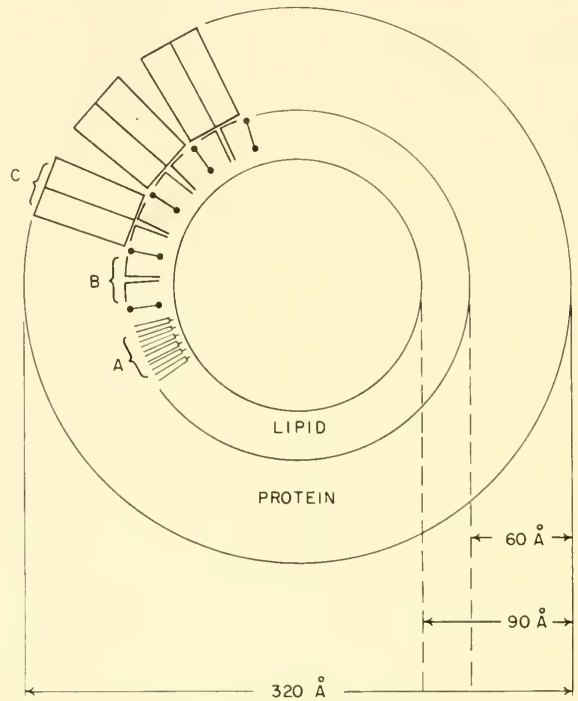

Fig. 11. A working hypothesis of the ultrastructure of the chromatophore. The Chromatium chromatophore is described as a hollow sphere about $320 \AA$ in diameter with a cortex about $90 \AA$ thick. The pigment molecules (B) aligned in a monolayer are bounded internally by a phospholipid monolayer ( $A$ ) and externally by a 60 - $\AA$-thick protein layer. The "minimal unit" of composition has been used as a structural subunit. The protein has been folded and is related directly to two chlorophyll molecules. On the average, the protein is related indirectly to one carotenoid molecule and ten phospholipid molecules.

the lipid surface as $\alpha$-helices. Such an arrangement provides an opportunity for visualizing a specific relationship between the terminal amino acid sequence and the polar groups of the porphyrin. In addition, a particular spatial orientation could be specified by the pitch of the helix. Disulfide bridges between the subunits would account for the observed stability of the chromatophore. 
A working hypothesis should represent a balance between simplicity and concrete suggestions. Our simplified model (Fig. 11) satisfies this criterion, but testing by prediction is needed to assess its utility.

\section{Note Added in Proof}

W. Arnold and R. K. Clayton have obtained evidence with chromatophore films (Proc. Nat. Acad. Sci. U.S. 46: 769-776; 1960) that the first step in photosynthesis appears to be the separation of an electron and a hole in a chlorophyll semiconductor. The data support our hypothesis of the ultrastructure of the chromatophore.

\section{References}

Anderson, I. C., and R. C. Fuller. 1958. Photophosphorylation by isolated chromatophores of the purple sulfur bacteria. Arch. Biochem. Biophys. 76: 168-179. Anderson, I. C., R. C. Fuller, and J. A. Bergeron. 1958. Environmental effects on photophosphorylation by bacterial chromatophores. Plant Physiol. 33: Suppl. XVII.

Arnon, D. I. 1958. Chloroplasts and photosynthesis. Brookhaven Symposia in Biol. No. 11: 181-235.

Arnon, D. I., M. B. Allen, and F. R. Whatley. 1954a. Photosynthesis by isolated chloroplasts. Nature 174: 394-396.

Arnon, D. I., F. R. Whatley, and M. B. Allen. 1954b. Photosynthesis by isolated chloroplasts. II. Photosynthetic phosphorylation, the conversion of light into phosphate bond energy. J. Am. Chem. Soc. 76: 6324-6328.

Bergeron, J. A. 1958. The bacterial chromatophore. Brookhaven Symposia in Biol. No. 11: 118-131.

Bergeron, J. A., I. C. Anderson, and R. C. Fuller. 1957. Studies on the chromatophore of the purple sulfur bacteria. Plant Physiol. Suppl. XVI. Vol. 32.

Bergeron, J. A., and R. C. Fuller. 1959. The influence of carotenoids on the infrared spectrum of bacteriochlorophyll in Chromatium. Nature 184: 1340-1341.

Duysens, L. N. M. 1952. Transfer of excitation energy in photosynthesis. Thesis. State University of Utrecht, Netherlands.

Emerson, R. L., J. F. Stauffer, and W. W. Umbreit. 1944. Relationship between phosphorylation and photosynthesis in Chlorella. Am. J. Botany 31: 107-120.

French, C. S. 1940. The pigment-protein compound in photosynthetic bacteria. I. The extraction and properties of Photosynthin. J. Gen. Physiol. 23: 469-481.

Frenkel, A. W. 1954. Light induced phosphorylation by cell-free preparations of photosynthetic bacteria. J. Am. Chem. Soc. 76: 5568-5569.

FRENKel, A. W. 1959. Light induced reactions of bacterial chromatophores and their relation to photosynthesis. Ann. Rev. Plant Physiol. 10: 53-70.

Frey-Wyssling, A. 1953. Submicroscopic morphology of protoplasm. 2d English ed. Elsevier Publishing Co., Amsterdam.

Fuller, R. C., and I. C. Anderson. 1957. Photophosphorylation in purple sulfur bacteria. Plant Physiol. 32: Suppl. XVI.

Fuller, R. C., and I. C. Anderson. 1958. Suppression of carotenoid synthesis and its effect on the activity of photosynthetic bacterial chromatophores. Nature 181 : 252-254. 
Goodwin, T. W., and D. G. LAnd. 1956. The carotenoids of photosynthetic bacteria. Arch. Mikrobiol. 24: 305-312.

Goodwin, T. IV., and H. G. Osman. 1954. Studies in carotenogenesis. 10. Spirilloxanthin synthesis by washed cells of Rhodospirillum rubrum. Biochem J. 56: 222-230.

KAtZ, E., and E. C. WAssink. 1939. Infrared absorption spectra of chlorophyllous pigments in living cells and in extra-cellular states. Enzymologia 7: 97-112.

Newton, J. W. 1958. Immunochemical reactions of the photosynthetic apparatus in purple bacteria. Brookhaven Symposia in Biol. No. 11:289-295.

Newton, J. W., and M. D. Kamen. 1957. Photophosphorylation by sub-cellular particles from Chromatium. Biochim. et Biophys. Acta 25: 462-474.

Newton, J. W., and G. A. Newton. 1957. Composition of the photoactive subcellular particles from Chromatium. Arch. Biochem. Biophys. 71: 250-265.

Palade, G. E. 1952. A study of fixation for electron microscopy. J. Exptl. Med. 95: 285-298.

Pardee, A. B., H. K. Schachman, and R. Y. Stanier. 1952. Chromatophores of Rhodospirillum rubrum. Nature 169: 282-283.

Rabinowitch, E. I. 1945. Photosynthesis and Related Processes. Interscience Publishers, Inc., New York. Vol. 1.

Rabinowitch, E. I. 1951. Photosynthesis and Related Processes. Interscience Publishers, Inc., New York. Vol. 2, part. 1.

Rabinowitch, E. I. 1956. Photosynthesis and Related Processes. Interscience Publishers, Inc., New York. Vol. 2, part 2.

Ruben, S. 1943. Photosynthesis and phosphorylation. J. Am. Chem. Soc. 65: 279282.

Schachman, H. K., A. B. Pardee, and R. Y. Stanier. 1952. Studies on the macromolecular organization of microbial cells. Arch. Biochem. Biophys. 38: 245-260.

Sjöstrand, F. S. 1959. Fine structure of cytoplasm: The organization of membranous layers. Revs. Modern Phys. 31: 301-318.

Stanier, R. Y. 1958. Formation and function of the photosynthesizing pigment system in purple bacteria. Brookhaven Symposia in Biol. No. 11: 43-53.

Stoeckenius, W. 1959. An electron microscope study of myelin figures. J. Biochem. Biophys. Cytol. 5: 491-500.

Thomas, J. B. 1952. A note on the occurence of grana in algae and in photosynthesizing bacteria. Koninkl. Ned. Akad. Wetenschap. Proc. Ser. C. 55: 207-208.

Trurnit, H. J., and G. Colmano. 1959. Chloroplast studies I. Absorption spectra of chlorophyll monolayers at liquid interfaces. Biochim. et Biophys. Acta 31: 434447.

van Niel, C. B. 1931. On the morphology and physiology of the purple and green sulphur bacteria. Arch. Mikrobiol. 3: 1-112.

van Niel, C. B. 1935. Photosynthesis of bacteria. Cold Spring Harbor Symposia Quant. Biol. 3: 138-150.

van Niel, C. B. 1936. On the metabolism of the Thiorhodaceae. Arch. Mikrobiol. 7: 323-358.

van Niel, C. B. 1956. In A. J. Kluyver and C. B. van Niel, The Mitrobes contribution to Biology. Harvard University Press, Cambridge, Mass. Chap. iii.

Vatter, A. E., H. C. Douglas, and R. S. Wolfe. 1959. Structure of Rhodomicrobium vanniclii. J. Bacteriol. $77: 812-813$.

VAtter, A. E., and R. S. Wolfe. 1958. The structure of photosynthetic bacteria. J. Bacteriol. 75: 480-483.

Williams, A. M. 1956. Light-induced uptake of inorganic phosphate in cell-free extracts of obligately anaerobic photosynthetic bacteria. Biochim. et Biophys. Acta 19: 570.

WOLKen, J. J. 1956. Photoreceptor structures. I. Pigment monolayers and molecular weight. J. Cellular Comp. Physiol. 48: 349-369.

Wolken, J. J., and F. A. Schwertz. 1956. Molecular weight of algal chloroplastin. Nature 177: 136-138. 


\section{DISCUSSION}

D. Waugh, J. A. Bergeron, A. C. Giese, M. V. Edds, Jr.

Dr. WAUgh (Massachusetts Institute of Technology): The properties found for the chromatophores themselves prompt me to ask about the physical and chemical properties of the protein component alone.

Dr. Bergeron: Our knowledge hasn't advanced sufficiently for such a study. However, the results of our initial attempts to obtain subunits by lysing the chromatophores with disulfide-cleaving agents have been promising. If we succeed in obtaining preparations which fulfill the criteria for subunits, then we will be in a better position to study the protein itself.

Dr. Giese (Stanford University): Does the proportion of one pigment to another in the chromatophore vary with the quality of the light, or with other conditions under which the culture is grown?

Dr. Bergeron: As you know, the colored-carotenoid:bacteriochlorophyll ratio can vary considerably. Since Stanier's evidence (1958) indicates that under certain conditions colorless precursors are transformed in situ, it is conceivable that there is a fixed ratio between bacteriochlorophyll and the $\mathrm{C}_{40}$ compounds, without specifying the degree of unsaturation. We believe that the protein-bacteriochlorophyll relationship probably sets a limiting value, with local conditions determining the kind and amount of carotenoids which are built into the chromatophore.

Dr. EDDs (Brown University): Are you able to tell us anything about the development of chromatophores?

Dr. Bergeron: We know very little about the development of bacterial chromatophores. There is the evidence that chromatophores form de novo in cells of Rhodospirillum rubrum when these organisms are induced to photosynthesize after a period of total dependence upon aerobic metabolism in the dark. We cannot perform similar experiments with Chromatium, which is an obligate anaerobic phototroph.

If chromatophores can appear in a cytoplasm which has been devoid of these structures, then we can assume either that the new chromatophores are derived by proliferation from other structures, such as the plasma membrane, or the larger vesicles, or that the chromatophores are the morphological consequence of the "homing instinct" of molecular subunits. Although, as our model indicates, we favor the idea of the subunit, it should be pointed out that immunochemical data have been employed by Newton (1958) to infer a specific relationship between the surface of the organism and the chromatophores, and we have observed evaginations from the larger vesicles. These evaginations, if severed, would be morphologically indistinguishable from the chromatophores. 



\title{
Polysaccharide-Protein Complexes of Yeast Cell Walls
}

\author{
Walter J. Nickerson, ${ }^{2}$ G. Falcone, ${ }^{3}$ and Gian Kessler ${ }^{4}$
}

Rapid advances in our knowledge of the chemical composition of the cell walls of microorganisms have resulted from the development of techniques for obtaining cell-wall preparations free from other cellular components, and from the establishment of criteria of homogeneity of cell-wall preparations. Microbial cells may be ruptured by mechanical or enzymatic procedures, and the broken cells may be separated from intact cells by centrifugation at low speed. By differential centrifugation and repeated washing of the cell-wall fraction, clean cell-wall preparations may be obtained that appear morphologically uniform in the electron microscope. The dialyzed and lyophilized wall preparations may be fractionated and analyzed with a fair degree of confidence that the results will not be negated by contamination from cytoplasmic debris.

The cell wall comprises about 30 per cent of the dry weight of bakers' or brewers' yeast, and includes a fraction which is remarkably resistant to chemical or enzymatic attack. In fact, the insoluble residue obtained on toluene autolysis of yeast, and the insoluble residue remaining after treating yeast with $1 \mathrm{~N} \mathrm{NaOH}$ at $100^{\circ} \mathrm{C}$, retains much of the shape of a yeast cell. Early investigations drew attention to the polysaccharide nature of this residue, and deduced that the cell wall of yeast comprised "yeast cellulose" and "yeast gum."

${ }^{1}$ Contribution from the Institute of Microbiology, Rutgers, The State University, New Brunswick, New Jersey; experimental work described supported in part by grants from the U. S. Public Health Service. The authors express their appreciation to Pauline E. Holbert (Institute of Microbiology, Rutgers, The State University) for her collaboration in the preparation of electron micrographs, and to Dr. D. E. Williams (Merck \& Co.; Rahway, N. J.) for his collaboration on sedimentation analyses.

${ }^{2}$ Rutgers, The State University, New Brunswick, N. J.

${ }^{3}$ Istituto di Patologia Generale, Universitá, Naples, Italy.

${ }^{4}$ Department of Biochemistry, University of California, Berkeley, Calif. 


\section{Extraction of Wall Components from Intact Cells}

In view of the substantial body of information developed up to about 1950 on the polysaccharides obtained by extraction of intact yeast cells with hot water, hot alkali, or cold concentrated acid, it seems advisable to present a short survey of information available prior to the present era of studies on the composition of clean cell walls isolated from mechanically disrupted yeasts. A comparison of results obtained by application of the several methods of extraction enumerated above has been presented by Garzuly-Janke (1940), who concluded that hot alkaline extraction procedures lead to more or less profound modifications of the alkali-soluble polysaccharide component. Certainly the application of more or less drastic extraction procedures designed to remove a polysaccharide material from a cell as a protein-free, nucleic acid-free moiety has led us to some insight into the nature of the polysaccharide in question, but it has also given rise to considerable controversy about the natural structure of the polysaccharide. Furthermore, efforts directed at "throwing away the protein" have not led to an appreciation of the importance of protein-polysaccharide complexes as the macromolecular fibers of the cell-wall fabric.

Yeast Glucan. The water-insoluble polysaccharide fraction of yeast that resists boiling in dilute alkali was studied by Salkowski (1894b) and designated yeast "achroocellulose." This fraction was subsequently studied by Zechmeister and Toth (1934), who found that, like cellulose, the resistant polysaccharide could be hydrolyzed in 40 per cent $\mathrm{HCl}$, although it did not exhibit many of the other characteristics of cellulose (yeast glucan is insoluble in Schweizer's reagent, does not produce a color with zinc-chlor-iodide, and does not yield cellobiose on acetolysis). An uncommon 1,3-glucosidic linkage was indicated for yeast glucan from methylation studies: 2,4,6-trimethylglucose was the sole product isolated. Further work by Hassid et al. (1941) on the glucan fraction confirmed 2,4,6-trimethylglucose as the sole methylation product, and supplied evidence of the $\beta$-configuration for the anhydroglucose units in the polymer (i.e., low specific rotations of the alkylated derivatives and upward mutarotation during their hydrolysis). From viscosity determinations a molecular weight of 6500 was suggested, and the 
glucan was characterized as a presumably closed-chain polymer of $\beta$-1,3-glucosido-glucopyranose units.

The structure of yeast glucan was reinvestigated by Bell and Northcote (1950). Mildly acidic conditions (0.5 N acetic acid), in place of strong mineral acid, were employed to effect isolation of the polymer, and chromatographic procedures were used to detect hydrolysis products following exhaustive methylation (former methods relied on refractive indices and methoxyl content of the fractions obtained by distillation of the hydrolyzed methylated glucan). With these refinements, 2,3,4,6-tetramethyl-D-glucose and 4,6-dimethyl-Dglucose were detected, in addition to 2,4,6-trimethyl-D-glucose, as products of hydrolysis of the methylated glucan. The methylated derivatives of $\mathrm{D}$-glucose were present in a ratio of tetra:di:tri $=1: 1: 9$, thus necessitating a drastic revision of the structural formula that could be considered for glucan. Bell and Northcote considered the polymer to be a highly branched structure in which each unit chain consists, on the average, of nine anhydroglucose radicals.

Glucomannans of Yeast. Early studies on the so-called yeast gum showed it to contain glucose and mannose. Purified preparations with $[a]_{\mathrm{D}}{ }^{20}=+88.5^{\circ}$ were obtained by Salkowski (1894a), Meigen and Spreng (1908), and von Euler and Fodor (1911).

The preparation obtained by Meigen and Spreng had a ratio of mannose: glucose $=2: 1$, whereas that of von Euler and Fodor showed a ratio of $1: 1$. In the latter case, the gum was obtained by extracting fat-free yeast with boiling water, or by precipitating the polysaccharide with ethanol from an autolysate of brewers' yeast. The polysaccharide was purified by repeated precipitation of the copper complex, according to the technique of Salkowski, and subsequent dialysis. It should be noted that treatment with hot alkali was avoided. Quantitative analyses for mannose in the presence of glucose in acid hydrolysates of the polysaccharide were carried out by differential precipitation of the mannose phenylhydrazone.

Subsequent investigations by Harden and Young (1912) and by Ling et al. (1925) employed an extraction step involving boiling 2 per cent $\mathrm{NaOH}$, and subsequent treatment of the alcohol-precipitable material with 60 per cent $\mathrm{KOH}$ for 2 hours in a vigorously stirred boiling-water bath.

The structure of a yeast mannan that was presumed to yield only D-mannose on acid hydrolysis was studied by Haworth and collab- 
orators (1937, 1939, 1941). Exhaustive methylation of alkaline solutions of the polysaccharide resulted in the formation of $2,3,4,6$ tetramethyl-, 3,4-dimethyl-, and 2,4,6-trimethylmannopyranose derivatives. The occurrence of a 3,4-dimethyl derivative indicated a double linkage to other components of the polymer. The evidence was assessed as indicating that the polymer consists of a main chain of 1,6-a-mannopyranose units to which are joined short side-chains at the number 2 carbon of alternate rings. It should be pointed out that the mannan employed in these studies was extracted from bakers' yeast by boiling in 6 per cent $\mathrm{NaOH}$ solution for 8 hours. Furthermore, it must be noted that the yield of mannose phenylhydrazone from acid-hydrolyzed purified mannan was actually only 50 per cent of the theoretical amount. When a correction (based on the recovery of only 82 per cent of the theoretical amount of mannose phenylhydrazone from pure mannose) was applied, the claim was made that a minimum yield of 92 per cent of the theoretical quantity of mannose was obtained on the hydrolysis of mannan. Actually, even allowing correction for failure to recover the theoretical quantity of phenylhydrazone from a known solution of mannose, it would seem that the hydrolysate of mannan did not contain more than two-thirds of the theoretical amount of mannose.

\section{Wall Components of Mechanically Disintegrated Yeasts}

Publications appeared independently by Northcote and Horne (1952) and by Houwink et al. (1951) reporting the localization of glucan, mannan, and chitin as components of isolated cell-wall fragments. After mechanical rupture of cells, Northcote and Horne isolated washed cell walls of bakers' yeast. This material was free of whole cells and cell debris and comprised the outer membranes of the cell; it was completely Gram-negative in character, in contrast to whole yeast cells which normally are intensely Gram-positive. The cell wall was found to include nitrogenous substance and lipid and to be composed principally of two polysaccharides-a mannan and a glucan. Glycogen was not associated with cell walls isolated by this procedure. Concurrent studies with the electron microscope showed that the isolated wall material consisted of at least two layers, the outer composed principally of glucan and the inner consisting of a nitrogenous substance and mannan. The over-all composition of the cell wall was taken to be: glucan 29 per cent, mannan 31 per cent, protein 13 per cent (based only on determination of total nitrogen), 
lipid (mainly neutral fat) 8.5 per cent, and ash 3 per cent, leaving 16 per cent unaccounted for by this procedure. Comparable data, in agreement with the above, were reported by Roelofsen (1953). Not all of the nitrogen found in yeast cell-wall preparations is associated with protein; many reports indicate the presence of chitin in veast cell walls. Employing the chitosan sulfate microchemical technique, Roelofsen and Hoette (1951) demonstrated the presence of chitin in bakers' yeast and other yeast species, thereby confirming observations of Schmidt (1936) and Nabel (1939), who had claimed the presence of chitin in yeast.

Preparation of Isolated Clean Cell Walls of Yeast. By mechanical disruption of bakers' yeast and Candida albicans, followed by

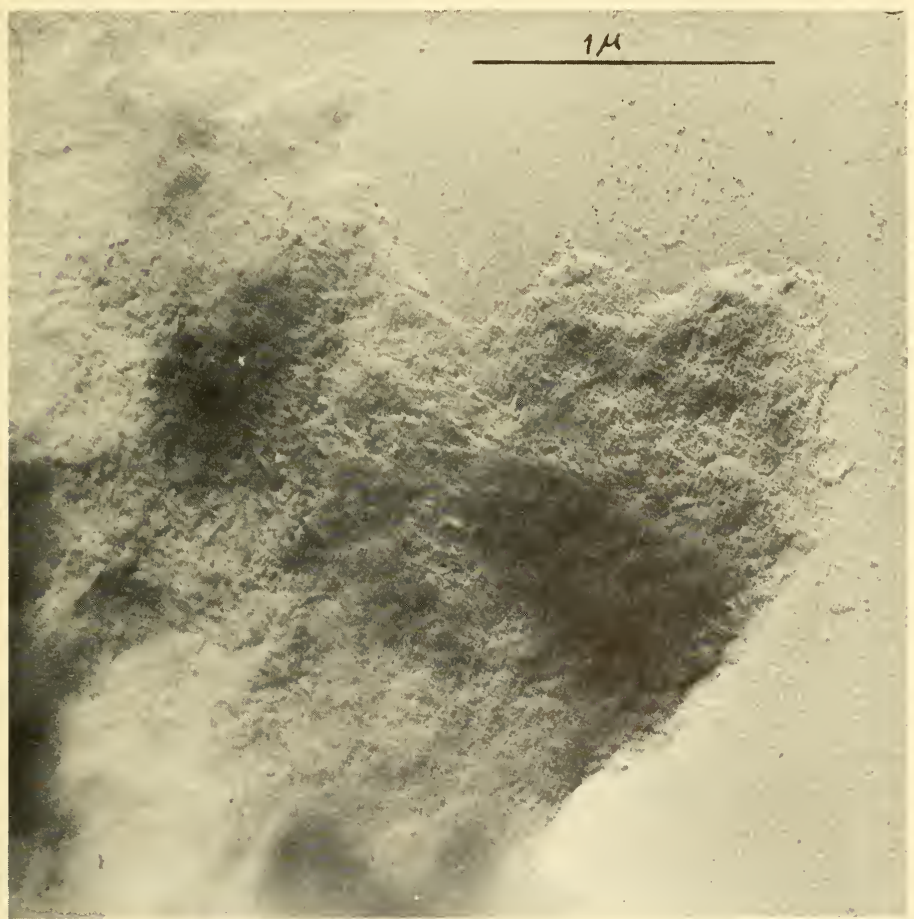

Fig. 1. Cell-wall fragment obtained by mechanical disintegration and differential centrifugation of Candida albicans. 
differential centrifugation in media of differing density, preparations of isolated cell walls were obtained by Falcone and Nickerson (1956) that were entirely free of intact cells and other particulate cellular matter. Cells from starch-free pound cakes of bakers' yeast (Anheuser-Busch) or laboratory-grown C. albicans were suspended in water or in a 5 per cent aqueous solution of dithioglycol ( $150 \mathrm{~g}$ yeast: $50 \mathrm{ml}$ solution) and were disrupted in the cold by agitation in a Waring Blendor with glass beads, according to the method of Lamanna and Mallette (1954). More than 90 per cent breakage of cells was achieved after 90 minutes' agitation.

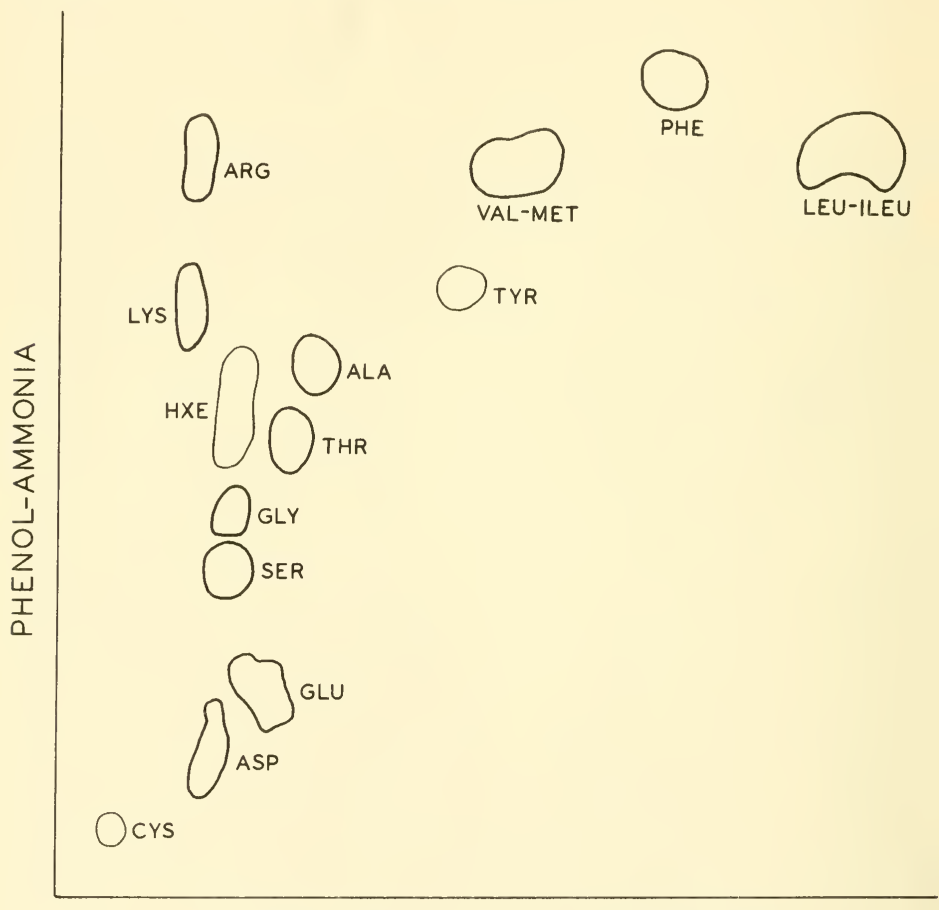

$$
\text { n-BUTANOL-ACETIC ACID-WATER }
$$

(a)

Fig. 2. Two-dimensional chromatograms of amino acids obtained on acid hydrolysis of isolated, clean cell walls (a) of Candida albicans, normal strain 582, and (b) of C. albicans, selenite-resistant mutant strain RM806, grown on a 
Cell-wall fragments were isolated by differential centrifugation and repeated washing with distilled water, 8.5 per cent sucrose solution, and phosphate buffer ( $\mathrm{pH} 7.4,0.1 \mathrm{M})$. IVashing with sucrose and with buffer served to eliminate completely the small particles which, in water, sediment along with the cell-wall fragments. After about 50 repetitions of washing and differential centrifugation, a fraction consisting exclusively of cell-wall material was obtained (Fig. 1). This was lyophilized, and the analytical data are based on material prepared in this manner. The nitrogen content of cell-wall preparations from two different batches of bakers' yeast

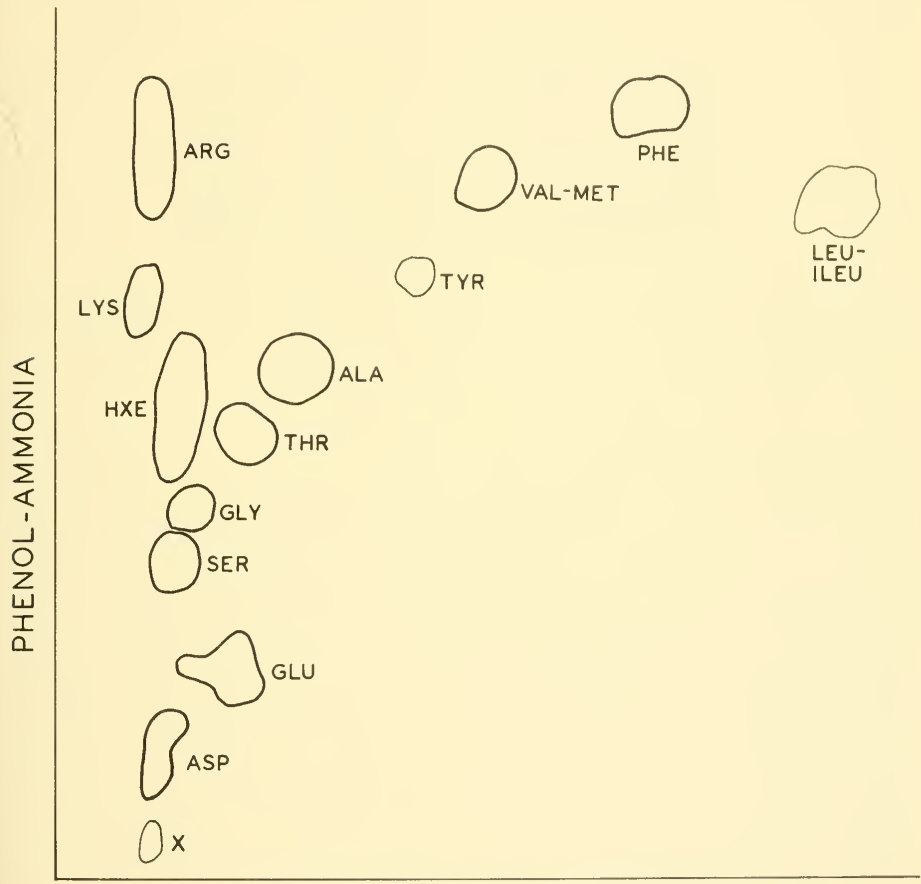

n-BUTANOL-ACETIC ACID-WATER

(b)

medium containing $10^{-3} \mathrm{M} \mathrm{Na}_{2} \mathrm{SeO}_{3}$. Note spot (X) in (b), corresponding to cysteic acid of (a), presumed to be a selenium-containing analogue. 
was 1.28 and 1.24 per cent. These values are lower than that (2.1 per cent) reported by Northcote and Horne (1952), who stated that their preparation contained no unbroken cells but was contaminated by small particles which were difficult to eliminate.

The analytical data show that the clean cell-wall material contains approximately 7 per cent protein, 85 per cent polysaccharide, and 3 per cent chitin. The high sulfur:protein ratio (2.1 per cent) indicated that the protein may be a pseudokeratin type. By means of two-dimensional paper chromatography of hydrolysates of cellwall material $\left(6 \mathrm{~N} \mathrm{HCl}\right.$ for 16 hours at $110^{\circ} \mathrm{C}$ ), the presence of 16 amino acids was detected. Representative chromatograms from cell walls of two strains of C. albicans are shown in Fig. $2 \mathrm{a}$ and b.

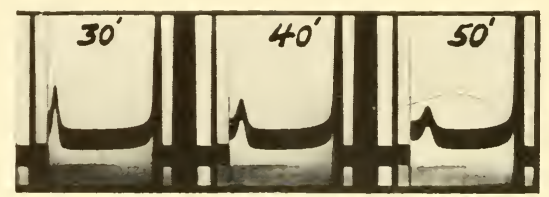

Fig. 3. Sedimentation pattern of primary glucomannan-protein (isolated from bakers' yeast cell wall) in analytical ultracentrifuge. Duration of centrifugation noted on each exposure. Note indication of low molecular weight material and apparent homogeneity of major peak.

From preparations of clean cell wall, the major fraction (approximately 75 per cent) was solubilized in $1 \mathrm{~N} \mathrm{KOH}$ on warming to about $50^{\circ} \mathrm{C}$. The solubilized material was dialyzed against running tap-water for 24 hours; on lyophilization, a white powder was obtained, of which the bulk was readily soluble in water. The watersoluble fraction was found to contain polysaccharide and protein, and was assumed to be a mannan-protein complex on the basis of the absence of the characteristic copper precipitate with Fehling's solution. This view was reinforced by ultracentrifugal studies which revealed the material to be monodisperse at several concentrations in water and in buffer. Representative sedimentation patterns are shown in Fig. 3, from which a sedimentation constant $S_{20}=4.3$ $\times 10^{-13}$ was calculated.

Separation of Glucomannan-Proteins from Isolated Cell Walls. The initial work on fractionation of cell-wall components led to recognition of their polysaccharide-protein nature and made clear the necessity for removal of lipid components; subsequent work 


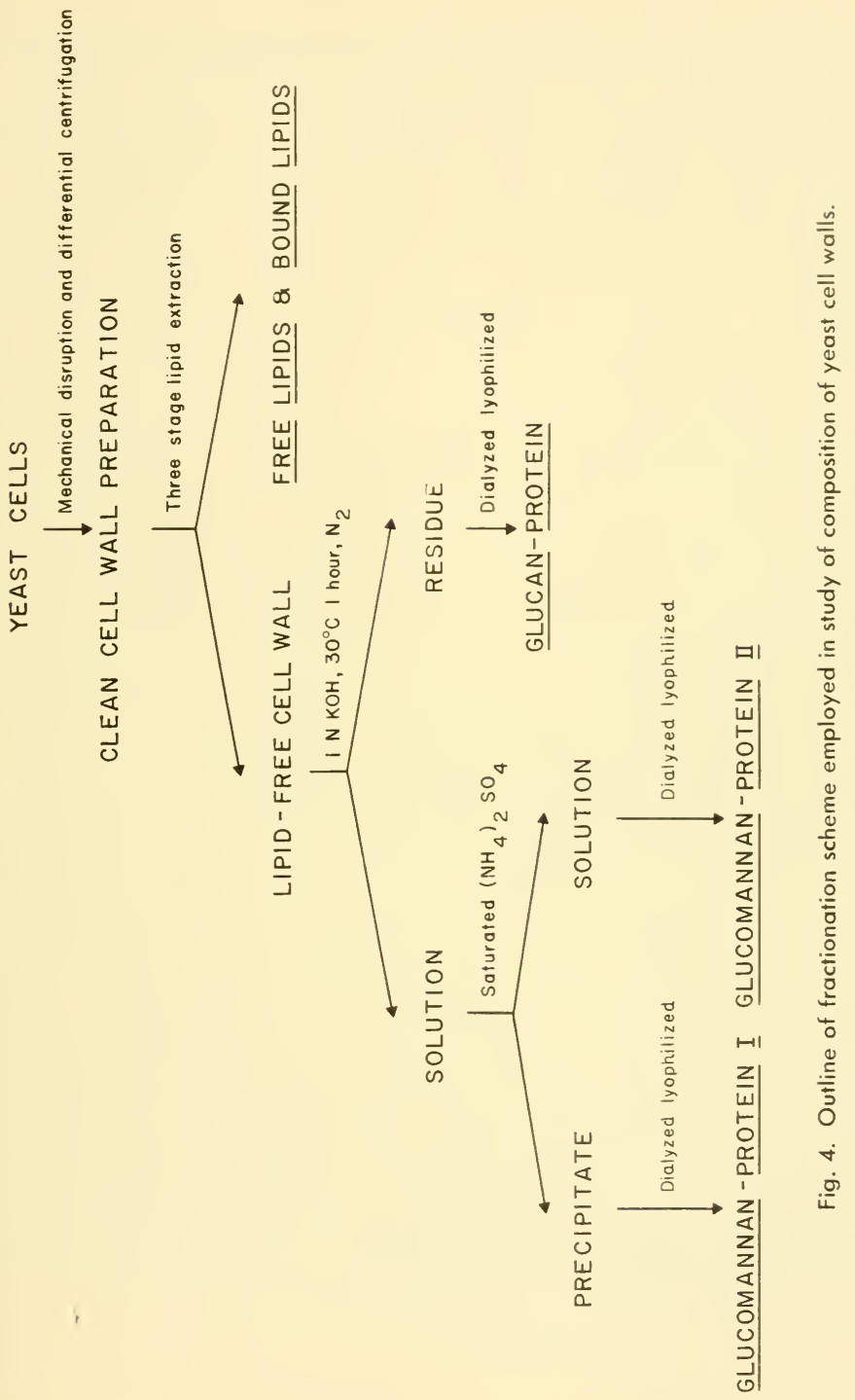


(Kessler and Nickerson, 1959) followed the scheme of fractionation shown in Fig. 4. Lipid-free cell-wall material was suspended in $1 \mathrm{~N}$ $\mathrm{KOH}$ for 1 hour under nitrogen at $25^{\circ} \mathrm{C}$; the solution obtained was passed through an ultrafine sintered glass filter. The filtrate was dialyzed for 24 hours against running tap-water and lyophilized. The resulting white powder was suspended in water at $25^{\circ} \mathrm{C}$ for 1 hour, then centrifuged. The centrifugate was saturated with ammonium sulfate; a precipitate was obtained that was removed by high-speed centrifugation. The precipitate and the supernatant were dialyzed, concentrated, and lyophilized. Both of the resulting white powders proved to be polysaccharide-protein complexes, with

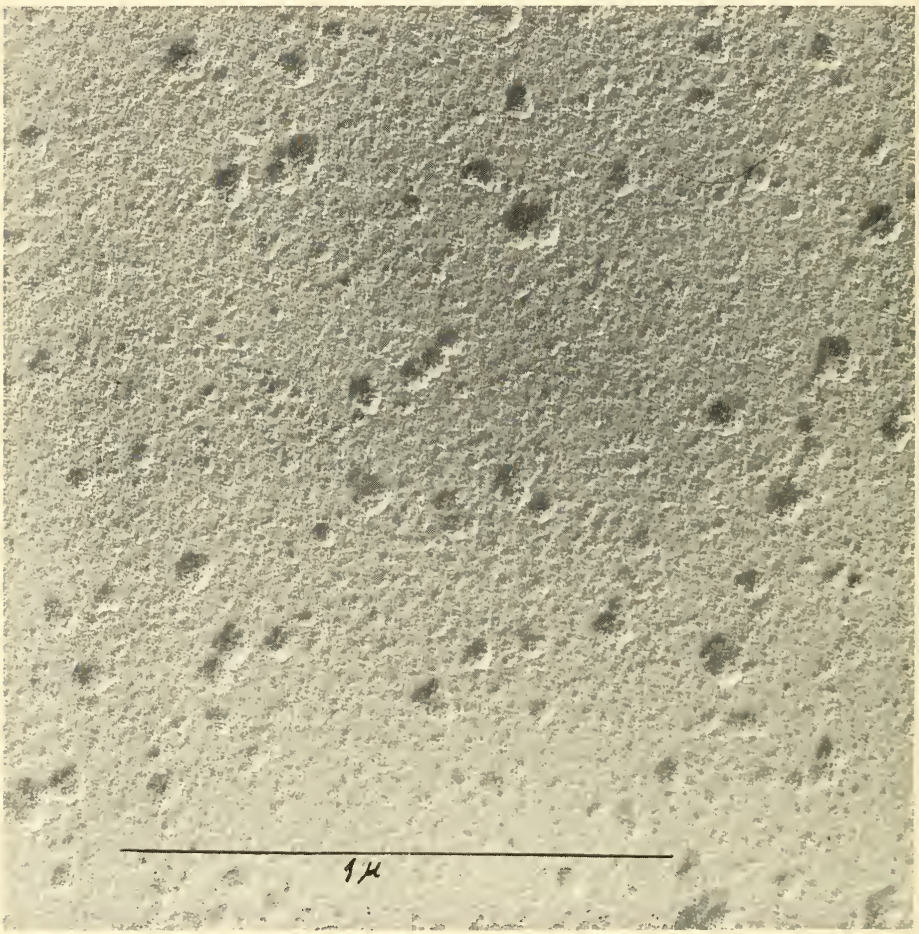

Fig. 5. Electron micrograph of glucomannan-protein I (GMP-I). Note aggregation of small spherical structures into globular masses. Deposited from solution onto Formvar film. 
mannose and glucose present in a ratio of approximately $1: 1$ in the fraction (termed glucomannan-protein I, or GMP-I) precipitated by saturated ammonium sulfate, and approximately $2: 1$ in the fraction (GMP-II) that remained in solution. The alkali-insoluble residue proved to be a third polysaccharide-protein fraction, the polysaccharide being composed very largely of glucose. This fraction, termed glucan-protein, comprised approximately 40 per cent of the weight of the wall material.

Examination of Wall Components by Physical Methods. The polysaccharide-protein fractions obtained in the manner just described were examined by a variety of physical and chemical means to ascertain their form, relative homogeneity, and composition. Electron micrographs of GMP-I, evaporated from solution onto Formvar membranes, are shown in Figs. 5, 6. The pronounced globular appearance of aggregates of GMP-I can be seen to arise from the clumping of much smaller spherical shapes that seem to possess an electron-dense center. In Fig. 6, a "structure" that has the size and shape of the inner portion of a bud scar is seen. This "scar" portion is clearly distinct from the "bud scar" of the outer

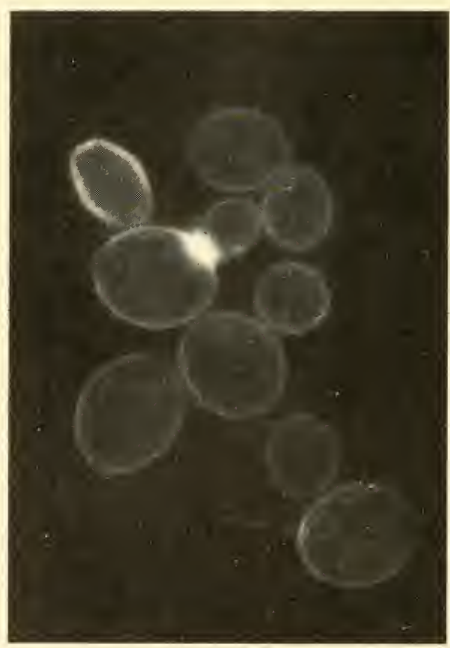

Fig. 7. Appearance of budding cells of bakers' yeast by aniline blue fluorescence microscopy. Note localization of oriented dye molecules in toroidal zone between bud and mother cell. (Courtesy, Currier, 1957.) 
glucan component of the wall; in the latter, the scar results from the circular ordering of fibrillar components into a highly crystalline pattern. The biochemical and mechanical sequences that operate during the budding process, and which bring about the circular orientation of fibrils in an otherwise highly disordered fibrillar network, have been discussed in detail elsewhere (Nickerson and Falcone, 1959; Falcone and Nickerson, 1959).

Evidence for the existence of a crystalline ringlike region in the living cell at the juncture of bud and mother cell can be seen (Fig. 7 ) from the work of Currier (1957) with aniline blue fluorescence

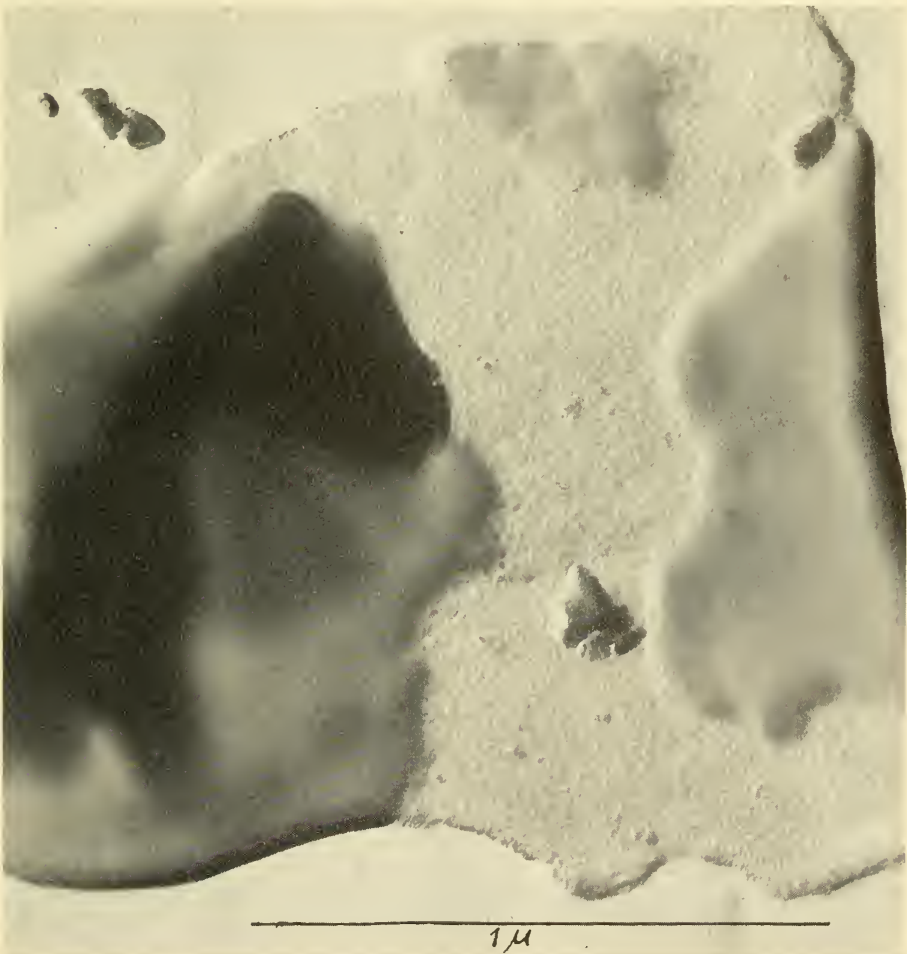

Fig. 8. Electron micrograph of isolated cell wall of bakers' yeast. Note difference in appearance between surface of outer layer and granular inner surface of wall. 
microscopy. In mechanically isolated wall fragments, the scar region (Figs. 8 to 10) gives evidence of morphological differences between outer and inner boundaries of the wall. The scar in Fig. 9, seen from "inside" the cell, appears to be composed of small spheres with dense centers (as seen in GMP-I in Fig. 5), and the plug in

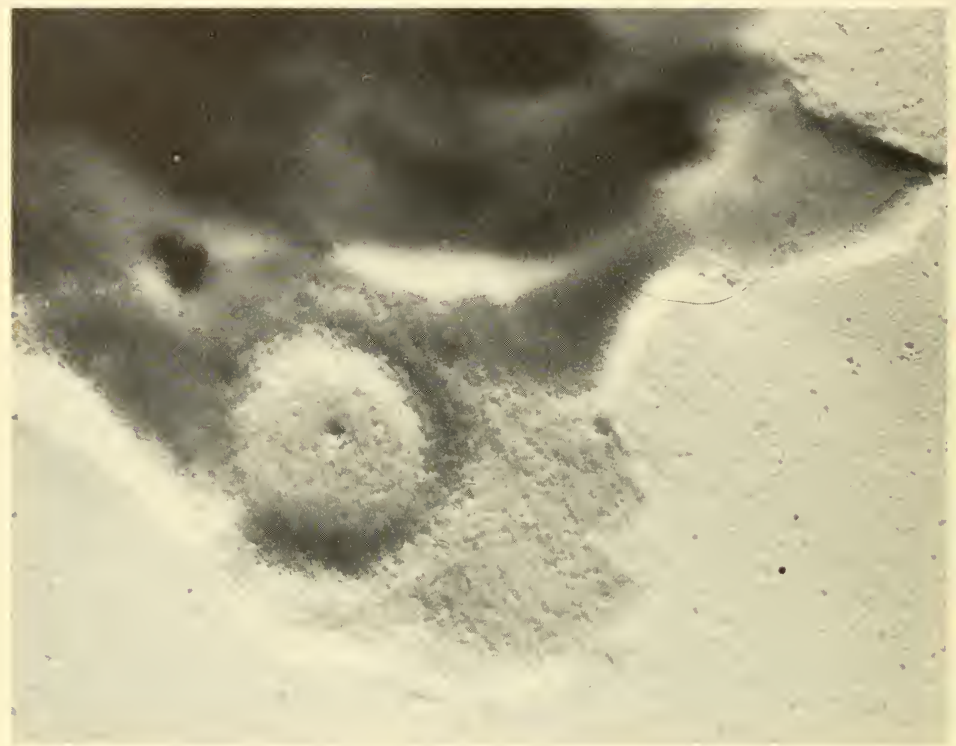

Fig. 9. Electron micrograph of isolated cell wall of bakers' yeast. Note bud scar as seen from inside cell; mark in center of scar appears as a small papilla. Scale as in Fig. 8.

the center of the scar appears as a papilla. In Fig. 10, the scar is seen from "outside" the cell, and the mark in the center of the scar appears as a depression.

On the matter of the existence of different layers in the cell wall of yeasts, Houwink and Kreger (1953) commented that none of their micrographs provided evidence that the cell wall consists of an inner and an outer layer. However, it should be pointed out that all except their "untreated" specimens were boiled in alkali, and they state, "Boiling with $3 \% \mathrm{NaOH}$ for three hours, on the assumption that it would prove useful to remove any remaining protein, leaves a 
thinner cell wall." Photographs of Northcote and Horne (1952) clearly show two layers in wall material that had been extracted only with lipid solvents, but their photographs reveal only one layer in wall material that had been treated with 3 per cent aqueous $\mathrm{NaOH}$ to remove mannan and protein.

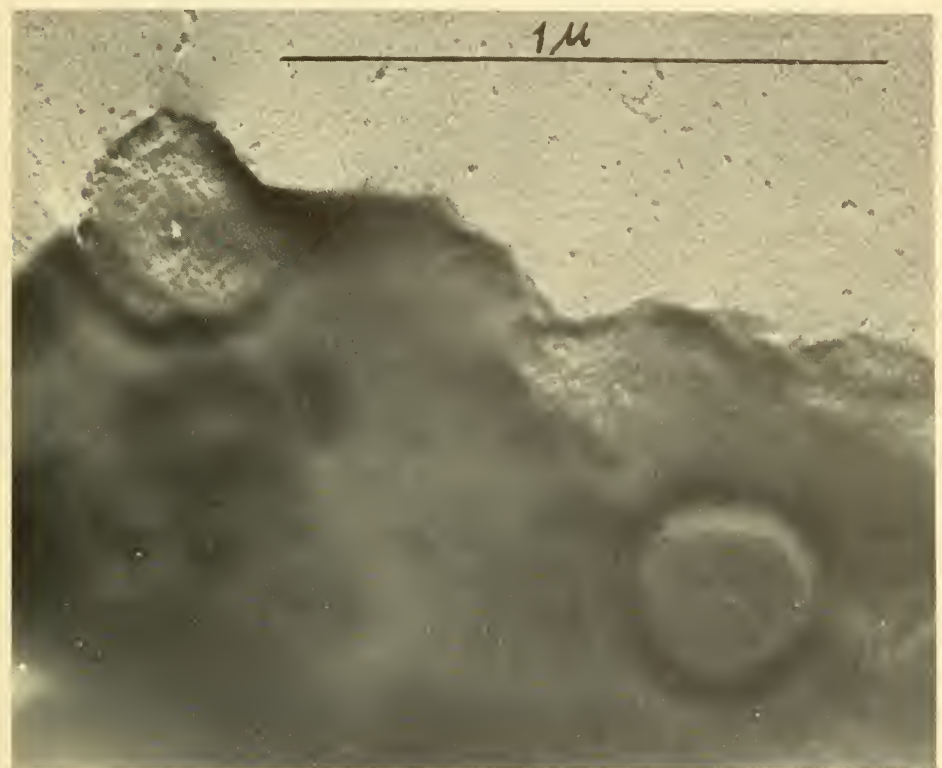

Fig. 10. Electron micrograph of isolated cell wall of bakers' yeast. Bud scar, as seen from outside of cell, has central mark that appears as a depression.

The initial ultracentrifugal studies on the glucomannan-protein complex in the primary alkaline extract of isolated walls had shown (Fig. 3) the presence of a major component that sedimented as a single peak, but the height of the boundary to the left of the peak indicated the presence of material of low molecular weight. On subsequent fractionation of the primary extract with ammonium sulfate, the components GMP-I and GMP-II were obtained. Ultracentrifugal studies on these components (Figs. 11, 12) show GMP-I to be a slowly sedimenting substance of low molecular weight, 
whereas GMP-II is responsible for the major peak observed in earlier sedimentation diagrams. On prolonged centrifugation, the G.MP-II diagram reveals the presence of two similar components (Fig. 12).
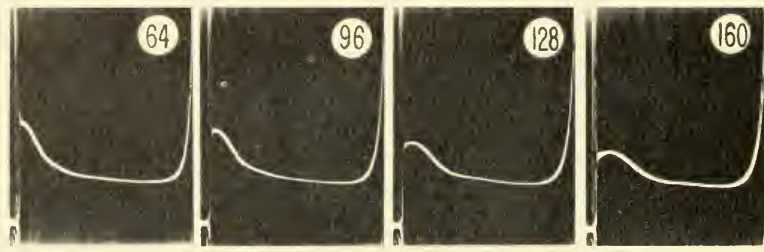

Fig. 11. Sedimentation pattern of glucomannan-protein I (GMP-I) in analytical ultracentrifuge. Material is evidently of low molecular weight.
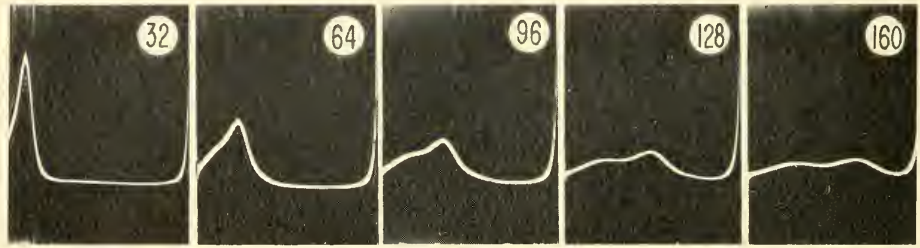

Fig. 12. Sedimentation of GMP-II in ultracentrifuge. Note correspondence to pattern of peak shown in Fig. 3. On prolonged centrifugation, evidence for two components is seen.

Analysis of Polysaccharide-Protein Complexes. Lipid-free wall preparations, subjected to the fractionation procedure shown in Fig. 4 , yielded recoveries of the individual complexes from the various yeasts as listed in Table 1. The glucan-protein fraction may account

\section{TABLE 1}

Polysaccharide-Protein Complexes of Yeast Cell Walls in Per Cent *

\begin{tabular}{llcc}
\hline \multicolumn{1}{c}{ Organism } & \multicolumn{1}{c}{ Glucan-Protein } & GMP-I & GMP-II \\
\hline Bakers' yeast $\ldots \ldots \ldots \ldots \ldots \ldots \ldots$ & 41.6 & 13.6 & 34.7 \\
S. cercvisiae $18.29 \ldots \ldots \ldots \ldots \ldots$ & 28.3 & 55.8 & 11.9 \\
C. albicans $582 \ldots \ldots \ldots \ldots \ldots$ & 47.4 & 3.0 & 27.2 \\
C. albicans $806 \ldots \ldots \ldots \ldots$ & 45.0 & 2.1 & 34.3 \\
\hline
\end{tabular}

- Data expressed as percentages of lipid-free wall recovered when fractionated according to scheme shown in Fig. 4; based on data of Kessler and Nickerson (1959). 
for approximately 40 per cent of the wall material, whereas the relative proportions of the two glucomaman-protein fractions vary from one organism to another.

On acid hydrolysis of the complexes and analysis for monosaccharide constituents, glucose and mamnose were found in the complexes in the percentages given in Table 2. The data indicate the presence of a glucan complex and of two glucomannan complexes in the cell wall. Closely similar carbohydrate composition was found in the three complexes from preparations of bakers' yeast, as well as from preparations of C. albicans.

TABLE 2

Relative Content of Glucose and Mannose in Cell Wall Components in Per Cent *

\begin{tabular}{|c|c|c|c|c|c|c|}
\hline \multirow[b]{2}{*}{ Organism } & \multicolumn{2}{|c|}{ Glucan-Protein } & \multicolumn{2}{|c|}{ GMP-I } & \multicolumn{2}{|c|}{ GMP-II } \\
\hline & Glucose & Mannose & Glucose & Mannose & Glucose & Mannose \\
\hline Bakers' yeast .... & 95 & 5 & 47 & 53 & 35 & 65 \\
\hline S. cerevisiae 18.29 & 95 & 5 & 45 & 55 & 33 & 67 \\
\hline C. albicans $806 \ldots \ldots$ & 95 & 5 & 48 & 52 & 37 & 63 \\
\hline C. albicans RM806 .... & 97 & 3 & 52 & 48 & 29 & 71 \\
\hline C. albicans $582 \ldots \ldots$ & 98 & 2 & 54 & 46 & 36 & 64 \\
\hline
\end{tabular}

- Data from Kessler and Nickerson ( 1959).

In all of the glucan preparations that have been isolated, a small amount of mannose has been detected. This is probably due to the fact that the mild alkaline treatment which is used for the fractionation of the wall preparations is not able to dissociate the glucomannans completely from the insoluble glucan skeleton. A prolonged exposure of the glucan to alkali can remove these traces of glucomannan, but at the same time this treatment causes a degradation or even a destruction of the protein that is associated with the carbohydrate material. Equal amounts of glucose and mannose are found in the material that can be precipitated from alkaline solution with ammonium sulfate, whereas mannose accounts for approximately two-thirds of the total sugar in the hydrolysate of the glucomannan complex II.

Chromatographic analysis revealed that the glucomannan-protein II of bakers' yeast is composed of 13 amino acids, the glucanprotein of 15 amino acids, and the glucomannan-protein I of 17 
amino acids. These findings hold for both the low-protein bakers' yeast and the high-protein strain (Saccharomyces cerevisiae, 18.29). Quantitative determinations on the amino acid composition of the protein components have been carried out on the three fractions; values for the recoveries of the principal amino acids are shown in Table 3 . These figures reveal that the proteins associated with the

TABLE 3

Amino Acid Analysis of Cell Wall Components *

\begin{tabular}{|c|c|c|c|}
\hline Amino Acid & Glucan-Protein $†$ & GMP-I $\ddagger$ & GMP-II \\
\hline Glutamic acid & 10.9 & 17.8 & 9.2 \\
\hline Aspartic acid & 29.8 & 13.1 & 31.1 \\
\hline Cysteic acid $\ldots \ldots \ldots \ldots$ & 1.5 & 0.2 & 8.4 \\
\hline Tyrosine $\ldots \ldots \ldots \ldots \ldots \ldots \ldots$ & 2.2 & 5.0 & 2.7 \\
\hline Serine $\quad \ldots \ldots \ldots \ldots \ldots \ldots \ldots$ & 4.1 & 4.1 & 4.6 \\
\hline Threonine $\ldots \ldots \ldots \ldots \ldots \ldots$ & 6.0 & 5.0 & 5.9 \\
\hline Glycine . & 3.3 & 3.5 & 4.6 \\
\hline Alanine $\ldots \ldots \ldots \ldots \ldots \ldots$ & 6.4 & 6.9 & 6.5 \\
\hline Valine $\ldots \ldots \ldots \ldots \ldots \ldots \ldots$ & 6.5 & 4.6 & 5.4 \\
\hline Proline $\ldots \ldots \ldots \ldots \ldots \ldots \ldots$ & 7.8 & 2.2 & \\
\hline Methionine $\ldots \ldots \ldots \ldots \ldots \ldots$ & & 1.6 & \\
\hline Isoleucine $\ldots \ldots \ldots \ldots \ldots \ldots$ & 4.5 & 5.1 & 4.9 \\
\hline Leucine $\ldots \ldots \ldots \ldots \ldots \ldots \ldots$ & 4.6 & 9.1 & 7.0 \\
\hline Lysine $\ldots \ldots \ldots \ldots \ldots \ldots \ldots$ & 5.1 & 8.1 & 6.2 \\
\hline Histicline $\ldots \ldots \ldots \ldots \ldots \ldots \ldots$ & & 2.7 & \\
\hline Arginine $\quad \ldots \ldots \ldots \ldots \ldots \ldots$ & 3.7 & 7.2 & 3.5 \\
\hline Phenylalanine $\ldots \ldots \ldots \ldots \ldots \ldots$ & 3.6 & 3.8 & \\
\hline
\end{tabular}

- Values expressed as percentages of total recovery from acid hydrolysates of each component; from data of Kessler and Nickerson (1959).

f Glucan-protein isolated from bakers' yeast.

‡ Glucomannan-proteins 1 and II isolated from strain 18.29 of Saccharomyces cerevisiae.

three fractions are highly acidic; ghutamic and aspartic acids, together, account for 31 per cent (GMP-I), 35 per cent (GP), and 39 per cent (GMP-II) of the total recovery. Although total recoveries of approximately 75 per cent of the amino acid components were obtained for GP and GMP-I, it must be borne in mind that some destruction and alteration of amino acid residues could not be avoided under the conditions used for the protein hydrolysis; tryptophan, if present, would have been completely destroyed.

Configuration of Polysaccharides in Wall Components. Immunochemical studies on the cross-reactions of glucomannan-pro- 
teins I and II with antisera to pneumococcus and Salmonella typhi have been carried out by Heidelberger. Negative reactions with both GMP-I and -II in type II pneumococcus antiserum indicated the absence of $a-1,6$ or $a-1,4$ linkages, whereas strongly positive cross-reactions in type VIII antiserum with GMP-I indicate the presence of $\beta-1,4$ glycosidic linkages. A strongly positive crossreaction in this antiserum was also obtained with Iles glucomannan (Heidelberger and Rebers, 1958), from which a cellobiose moiety has been obtained on partial hydrolysis (Smith and Srivastava, 1956). In contrast, GMP-II showed little, if any, cross-reaction with the type VIII antiserum. It is, therefore, of considerable interest to note that yeast "mannan," obtained by hot alkaline extraction procedures, has not been found to cross-react with any pneumococcal antiserum (Heidelberger, 1959). Both GMP-I and -II also gave strongly positive cross-reactions in typhoid antiserum, as had been found previously for yeast "mannan" (Heidelberger and Cordoba, 1956 ).

Additional evidence against the presence of $a$-pyranose derivatives in glucomannan-protein I is provided by infrared absorption spectra. Barker et al. (1956) have shown that $\alpha$-pyranose residues absorb strongly at $844 \mathrm{~cm}^{-1}$ and $\beta$-pyranose derivatives absorb at about $890 \mathrm{~cm}^{-1}$. Examination of Fig. 13 reveals practically no absorption in the $844 \mathrm{~cm}^{-1}$ region by GMP-I, but significant absorption by GMP-II. On the other hand, GMP-I exhibits a small peak at $890 \mathrm{~cm}^{-1}$ which is not shown by GMP-II.

Bonding Between Polysaccharide and Protein. A thoroughly washed, clean cell-wall preparation does not leach out material on standing in water. This insolubility of the wall is in sharp contrast to the solubility of GMP-I and -II in water. Glucan is the insoluble polysaccharide component of the wall to which the soluble components are bound. The high content of acidic amino acids in the protein components of the wall fractions immediately suggests that protein and polysaccharide may be joined through ester linkage. This possibility is being explored through studies of infrared spectra, and of the hydroxylamine ester-cleavage reaction. It is not yet possible to assess quantitatively the relative contributions of ester linkage (or other covalent linkage) and hydrogen-bonding in uniting the various components of the wall together to form the complex wall structure, but some understanding of this union may be forthcoming in the near future. 

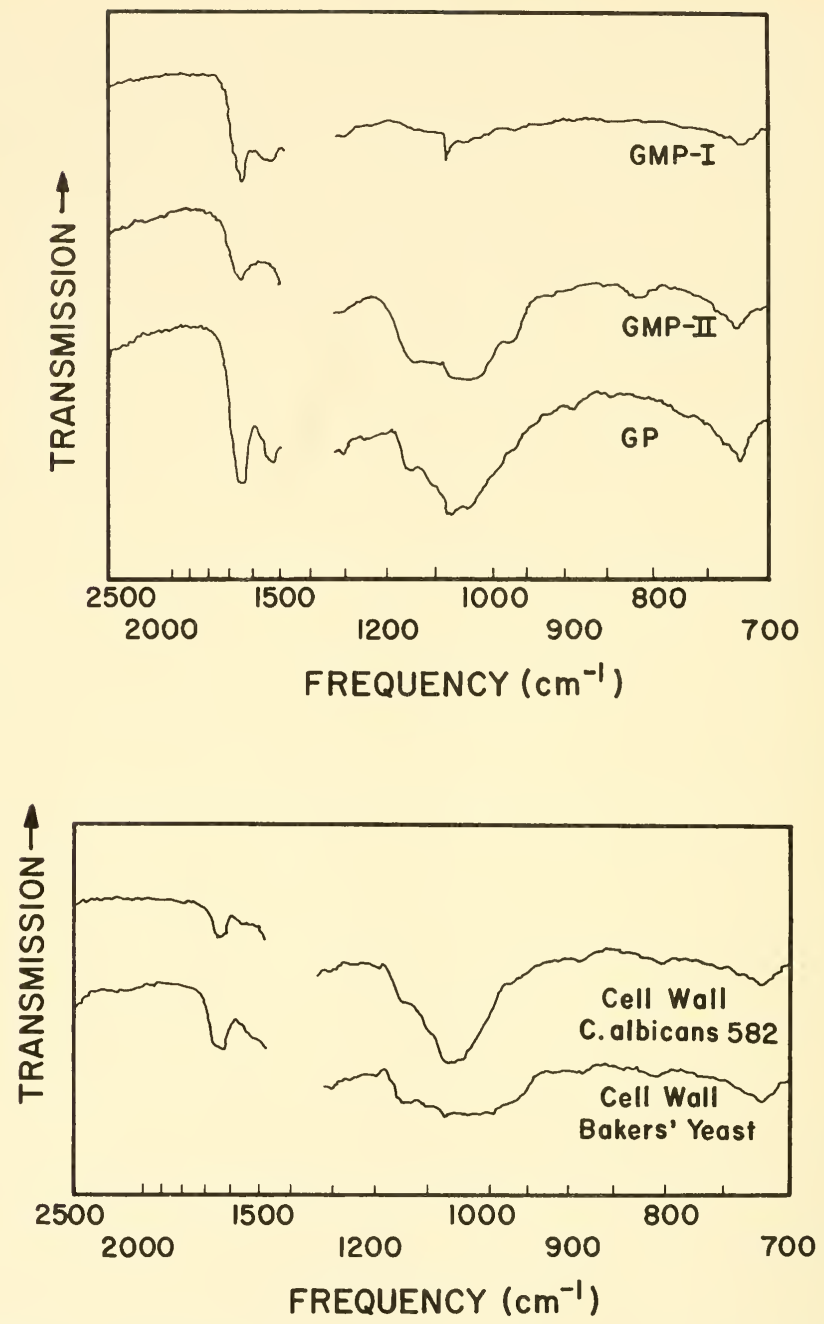

Fig. 13. (Above) Infrared absorption spectra for glucan-protein (GP). GMP-I, and GMP-II from high-protein strain 18.29 of Saccharomyces cerevisiae. (Below) Spectra obtained with isolated cell wall of bakers' yeast and C. albicans are shown for comparison. 


\section{Operation of Wall Components in Cellular Division}

The mechanism of cellular division in yeasts has been a subject of investigation in this laboratory for many years. It is intended at this time merely to indicate briefly the alterations in wall components that occur during the process of budding. From studies on divisionless mutant strains of Candida albicans it was found (Nickerson and Falcone, 1956) that an enzyme (termed "protein disulfide reductase," PDS reductase) which exists in normal strains causes reduction of disulfide bonds of the protein of glucomannan-protein. In the initial stages of this study, the primary glucomannan-protein was employed as substrate. (In an unfortunate typographical confusion, the primary glucomannan-protein, when used as substrate for PDS reductase, was referred to as GMP-I in one of our publications, Nickerson and Falcone, 1959.) In light of our recent fractionation of primary GMP, it is hoped that we will be able to identify more precisely the sulfur-rich substrate of PDS reductase.

The consequences of reduction of disulfide bonds in a wall component have been interpreted in terms of randomly disordered, covalent-bonded polymer matrices in which localized structural weakness is introduced by rupture of covalent bonds. At the weakened site, an explosive blowout occurs, for which evidence has been presented from short-interval, time-lapse photomicrography (Nickerson and Falcone, 1959). The naked protoplasmic sphere that is blown out of the mother cell constitutes the bud-initial. It rapidly becomes covered with wall substance by growth of the wall substance from the mother cell at the base of the bud-initial.

The force of the outward thrust at the instant of explosive budding serves to orient the fibrils of the glucan-protein and the spherical structures of GMP-I components, as shown in Figs. 6, 9, and 10 and discussed in detail elsewhere (Falcone and Nickerson, 1959). The breaking of covalent disulfide bonds within or between components of the wall fabric sets in motion the train of events witnessed in the budding process: the weakened wall cannot contain the internal pressure, a bud is extruded, and the macromolecular components, momentarily partially freed of bonding to neighboring components, are ordered by the outward fluid flow into the toroidal pattern that would be predicted from purely physical considerations. 


\section{References}

Barker, S. A., E. J. Bourne, and D. H. Whiffen. 1956. Use of infrared analysis in the determination of carbohydrate structure. Methods of Biochem. Anal. 3: 213245.

BARton, A. A. 1950. Some aspects of cell division in Saccharomyces cerevisiae. J. Gen. Microbiol. 4: 84-86.

Bell, D. J., and D. H. Northcote. 1950. The structure of a cell-wall polysaccharide of baker's yeast. J. Chem. Soc. Pp. 1944-1947.

Currier, H. B. 1957. Callose substance in plant cells. Am. J. Botany 44: 478-488.

FAlcone, G., and W. J. Nickerson. 1956. Cell-wall mannan-protein of baker's yeast. Science 124: 272-273.

FALCONE, G., and W. J. Nickerson. 1959. Enzymatic reactions involved in cellular division of microorganisms. In W. J. NrCKenson (ed.). Biochemistry of Morphogenesis. Pergamon Press, Ltd., London.

Garzuly-Janke, R. 1940. Über das Hefemannan. J. prakt. Chem. 156: 45-54.

Harden, A., and W. J. Young. 1912. The preparation of glycogen and yeast-gum from yeast. J. Chem. Soc. Pp. 1928-1930.

Hassid, W. Z., M. A. Joslyn, and R. M. McCready. 1941. The molecular constitution of an insoluble polysaccharide from yeast, Saccharomyces cerevisiae. J. Am. Chem. Soc. 63: 295-298.

Haworth, W. N., R. L. Неath, and S. Peat. 1941. The constitution of yeast mannan. J. Chem. Soc. Pp. 833-842.

Haworth, W. N., E. L. Hirst, and F. A. Isherwood. 1937. Polysaccharides. Part XXIV. Yeast mannan. J. Chem. Soc. Pp. 784-791.

Haworth, W. N., E. L. Hirst, F. Isherwood, and J. K. N. Jones. 1939. 2:3:4Trimethyl mannose. J. Chem. Soc. Pp. 1878-1880.

Heidel berger, M. 1959. All polysaccharides are immunologically specific. In M. L. IVolfrom (ed.). Carbohydrate Chemistry of Substances of Biological Interest. Pergamon Press, Ltd., London.

Heidelberger, M., and F. Cordoba. 1956. Cross-reactions of antityphoid and antiparatyphoid B horse sera with various polysaccharides. J. Exptl. Med. 104: 375382.

Heidelberger, M., and P. A. Rebers. 1958. Cross reactions of polyglucoses in antipneumococcal sera. VI. Precipitation of type VIII and type III antisera by $\beta$-glucans. J. Am. Chem. Soc. 80: 116-118.

Houwink, A. L., and D. R. Kreger. 1953. Observations on the cell wall of yeasts. An electron microscope and x-ray diffraction study. Antonie van Leeuwenhoek. J. Microbiol. Serol. 19: 1-24.

Houwink, A. L., D. R. Kreger, and P. A. Roelofsen. 1951. Composition and structure of yeast cell walls. Nature 168: 693-694.

Kessler, G., and W. J. Nickerson. 1959. Glucomannan-protein complexes from cell walls of yeasts. J. Biol. Chem. 234: 2281-2285.

Lamanna, C., and M. F. Mallette. 1954. Use of glass beads for the mechanical rupture of microorganisms in concentrated suspensions. J. Bacteriol. 67: 503-504.

Ling, A. R., D. R. NanjI, and F. J. Paton. 1925. Studies on glycogen. Part I. The nature of yeast glycogen, its preparation, estimation, and its role in yeast metabolism. J. Inst. Brewing 13: 316-321.

Meigen, W., and A. Spreng. 1908. Über die Kohlehydrate der Hefe. Hoppe-Seyler's Z. physiol. Chem. 55: 48-73.

Nabel, K. 1939. Über die Membran niederer Pilze, besonders von Rhizidiomyces bivellatus nov. spez. Arch. Mikrobiol. 10: 515-541.

Nickerson, W. J., and G. Falcone. 1956. Identification of protein disulfide reductase as a cellular division enzyme in yeasts. Science 124: 722-723.

Nickerson, W. J., and G. Falcone. 1959. Function of protein disulfide reductase in cellular division of yeasts. In R. Benesch (ed.). Sulfur in Proteins. Academic Press, Inc., New York. 
Northcote, D. H., and R. IV. Honne. 1952. The chemical composition and structure of the veast cell wall. Biochem. J. 51:232-236.

Roelofsen, P. A. 1953. Yeast mannan, a cell wall constituent of baker's yeast. Biochem. et Biophys. Acta 10: 477-478.

Roelofsen, P. A., and I. Hoette. 1951. Chitin in the cell wall of yeasts. Antonie' van Leewwenhoek. J. Microbiol. Serol. 17: 297-313.

Salkowski, E. 1894a. Ueber die Kohlehydrate der Ilefe, Ber. deut. chem. Ges. 27: $497-502$.

Salkowski, E. 1894b. Ueber die Kohlehydrate der Hefe. II. Die Hefecellulose. Ber. deut. chem. Ges. 27:3325-3329.

SснміDт, M. 1936. Makrochemische Untersuchungen über das Vorkommen von Chitin bei Mikroorganismen. Arch. Mikrobiol. 7: 241-260.

Smith, F., and H. C. Srivastava. 1956. Acetolysis of the glucomannan of Iles mannan. J. Am. Chem. Soc. 78: 1404-1408.

von Euler, H., and A. Fodor. 1911. Zur Kenntnis des Hefengummis. HoppeSeyler's Z. physiol. Chem. 72: 339-346.

Zechieister, L., and G. Tотн. 1934. Über die Polyose der Hefemembran. Biochem. Z. 270: 309-316.

\section{DISCUSSION}

\section{J. W. Green, W. J. Nickerson, H. Ducoff, R. D. Preston, J. D. Bergeron}

Dr. Green (Rutgers University): During the rupture of the cell wall and explosive budding, is the plasma membrane ruptured? In osmotic hemolysis, the rupture of the red cell can be shown by rapid photography to occur, not explosively, but by slow swelling.

Dr. Nickerson: Judging from the appearance of the bud-initial, as seen in the dark-field time-lapse photomicrographs, its resemblance to a spherical protoplast-albeit of natural origin-leads me to suspect that the plasma membrane is intact.

Dr. Ducoff (University of Illinois): How rapid is the change from the filamentous to the non-filamentous form? Is there a "breakup" comparable to that seen in induced bacterial filaments?

Dr. Nickerson: I have never observed a "breakup" in the filamentous yeast comparable to that which we have studied in bacterial filaments. Under appropriate conditions, an inoculum of filamentous cells of a divisionless strain is "diluted out" by budding, to give a population of largely normal yeast cells. These revert to their filamentous phenotype when the condition promoting cellular division is removed.

Dr. Preston (University of Leeds): How sure are you that the hole associated with a bud scar is actually blown out? In the algae we are studying, the production of zoospores is followed by the production of a hole in the wall of the parent cell. Stripping through the wall, and electron microscope examination, suggest that these holes are made by progressive dissolution of the wall; only the last few lamellae are blown out by turgor pressure.

Dr. Nickerson: Our use of the term "explosive blowout" to describe the budding of yeast is based on two independent observations. First, the lapse of time between consecutive frames showing an intact wall in one and a cell with an extruded bud-initial in the second, is 30 seconds. Second, the orientation of fibrils in the wall components at the site of budding can only be 
achieved by outward thrust of liquid at considerable velocity. We are currently attempting to calculate a minimum velocity for the thrust to result in the observed crystalline orientation.

Dr. Bergeron (Brookhaven National Laboratory): Is the cross-linking in the bud scar irreversible with regard to the later formation of overlapping buds?

Dr. Nickerson: Apparently so. The photographs of Barton (1950), who first observed bud scars, show no overlapping, even on cells with numerous scars. Our electron micrographs also show no overlapping of bud scars. 


\title{
Cellulose-Protein Complexes in Plant Cell Walls
}

\author{
R. D. Preston ${ }^{1}$
}

\section{Introduction}

The walls of living plant cells consist in the main of a mixture of polysaccharides and their derivatives organized in such a way as to be chemically stable while allowing the mutual displacements necessary in the dimensional changes associated with growth. Except in the fungi, which will not be dealt with here, the structural element of these walls is the polysaccharide, cellulose, whose constitution will be examined below. This substance takes the form of long threads known as microfibrils, some 100-200 ^ wide (depending on the plant species-see refs. in Preston, 1952) and about half as thick (Preston, 1951) (Fig. 1), which are often oriented, either very roughly or with precision (Fig. 2). Both the synthesis and the orientation of these microfibrils must involve proteins, and it is the aim of this article to inquire how far present evidence will allow us to go in defining the cellulose-protein complex which may be involved. With few exceptions, those cell walls which have been examined have been found to contain proteins (Thimann and Bonner, 1933; Allsopp and Misra, 1940; Myers et al., 1956; Bishop et al., 1958; Northcote et al., 1958) and, though in some preparations much of the protein is derived from cytoplasmic debris, there is no doubt that some part of it occurs in the wall itself. Occasional reference has been made to the inclusion in cell-wall preparations of electron microscopically visible particles which appear to be cytoplasmic (Myers et al., 1956).

It seems probable that the protein content of the wall sensu strictu is low, perhaps not more than a few per cent. A celluloseprotein complex is to be expected only during cellulose deposition, after which most of the protein will be resorbed. Even this small percentage remainder in the wall is therefore significant.

${ }^{1}$ University of Leeds, Leeds, England. 

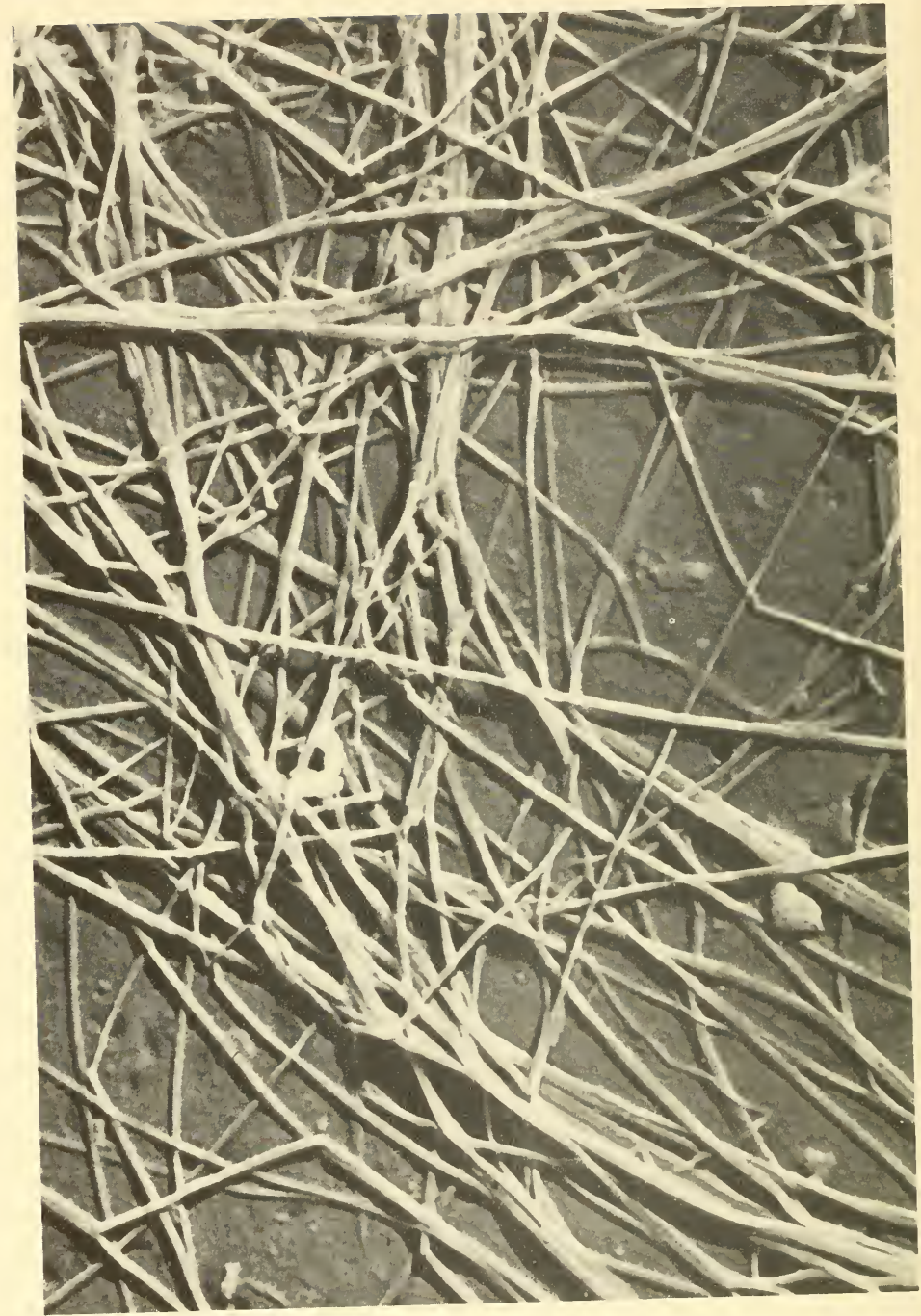

Fig. 1. Isolated microfibrils of Valonia cellulose. Shadowed Pd-Au. $\times 45,000$.

Fig. 1. Isolated microfibrils of Valonia cellulose. Shadowed Pd-AU. $\times 45,000$. 


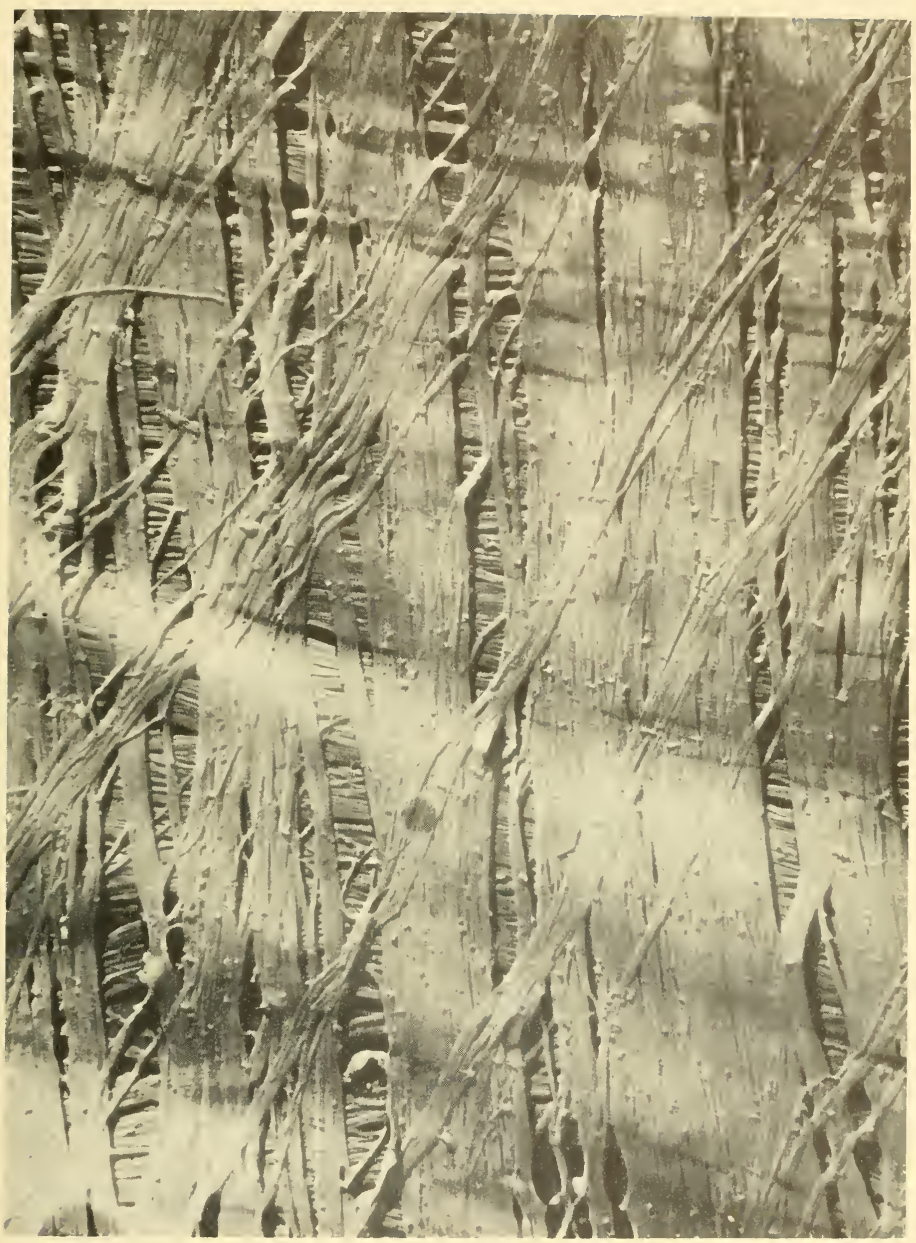

Fig. 2. Three wall lamellae in Valonia ventricosa. Note the two abundant sets of microfibrils lying almost at $90^{\circ}$ to each other and a third, less abundant, set lying obliquely. The lamellae are slightly torn. Shadowed Pd-Au. $\times 22,500$. 
Polysaccharide-protein complexes are beginning to acquire significance both in plant and in animal physiology; whether the structural component is dominantly protein or dominantly polysaccharide, it may well be that the same principles are involved. It is proposed here, therefore, to examine evidence from both fields.

Although the principal structural components of animal tissues are undoubtedly protein, there are many recorded observations which show that polysaccharides of several kinds are involved, sometimes in considerable abundance. An outstanding case is the development of tunicin, indistinguishable from plant cellulose, in the tunica of sea squirts, but many cases of polysaccharide association with proteins have been reported. These are often acid polysaccharides, the acidic group being represented by hyaluronic acid, chondroitin sulfuric acid, and some partially sulfated variants of these two. Some, however, are devoid of hexosamine or uronic acids. Grassman and Schleich (1935) identified glucose and galactose in hydrolysates of skin extracts, an observation confirmed by Gross et al. (1952), who added mannose as a constituent sugar. More recently, other polysaccharides rich in glucose have been reported from mammalian sources. Dische and Borenfreund (1954) claim to have demonstrated the presence in lens capsule of a polysaccharide containing galactose and glucose in the ratio 3:2, and Dische and Zelemenis (1955) found a similar polysaccharide in the vitreous body. Again, polysaccharide material containing galactose, mannose, and glucose in the ratio $6: 1: 2$ has been isolated from kidney (Dohlman and Balazs, 1955). It may be, however, that these represent mixtures of polysaccharides, since Dische et al. (1958) have found the composition of similar polysaccharides in the bones of cattle to vary with the age of the animal. Indeed, a polysaccharide fraction in abdominal subcutaneous tissue has been shown to consist of pure galactans and pure mannans (Consden and Bird, 1954). In all these cases, the polysaccharide is associated with a protein which is, in every case, collagen. There is no direct evidence that glucans alone exist in any of these tissues, but the hexasaccharide isolated from umbilical cord by Akiya and Tomoda (1955) and considered by them to consist of $\beta$-D-glucose residues joined by $1-4$ links, might be a precursor, or a degradation product, of cellulose.

There is some evidence that these polysaccharides play an important role in the stabilization of the associated collagen. Jackson 
(1953) and Wood (1954) have shown that a polysaccharide fraction resembling chondroitin sulfate is essential for the maintenance of some of the physical properties of collagen fibers. Similarly, Hall et al. (1952) and Hall (1955) have demonstrated that the removal of polysaccharides from elastic fibers leads to a marked change in the resistance of the fiber to chemical and enzymic attack. Moreover, for these fibers, particularly in the aorta, it has been claimed (Saxl, 1957) that pathological degradation is bound up with the topographical concentration of polysaccharides. Excessive resistance of connective tissue fibers to chemical attack has been ascribed to variations in the content of associated polysaccharide (Hall and Reed, 1957; Keech, 1958). Stabilization of this kind is regarded by $\mathrm{Yu}$ and Blumenthal (1958) as due to the existence of complexes between proteins and polysaccharides.

The more recent evidence in favor of a close relationship between cellulose and proteins in the cell walls of plants comes from four main sources. These will be dealt with separately below.

\section{Cellulose-Protein Complexes in Mammalian Tissue}

The recent demonstrations of the presence of cellulose in mammalian tissue (Hall and Reed, 1957) and of its association with protein in healthy and pathological skin (Hall et al., 1960; Hall and Saxl, 1961) gives a specific lead. The fibers concerned were first observed as highly birefringent inclusions in the alkaline degradation products of collagen (Burton et al., 1955; Hall et al., 1960). They were reported to be rich in polysaccharide and highly resistant to chemical attack, and the polysaccharide has been identified as cellulose for the following reasons: (1) The x-ray diagram is indistinguishable from that of cotton cellulose. (2) The fibrils of the polysaccharide appear to be uniaxial with refractive indices of 1.596 and 1.525 , very close to those of ramie fibers. (3) Paper-partition chromatography of hydrolysates reveals the presence of only traces of sugars other than glucose, and in particular no traces were found of glucosamine, galactosamine, glucuronic acid, or galacturonic acid. (4) The polysaccharide is soluble in Schweizer's reagent but not in hot water, alkali, or boiling dilute sulfuric acid. (5) The electron microsçope appearance and the electron-diffraction diagram closely resemble those of cellulose. 
The fibers consist of a core of protein, sometimes completely incased in polysaccharide. When the coating is not complete (Fig. 3), however, it can be seen to consist of fibrils running spirally round the fiber. The inclination of the fibrils to fiber length varies broadly around $72^{\circ}$ (cf., the value of $75^{\circ}$ in elongated, growing plant cellsPreston, 1947; Preston and Wardrop, 1949; Middlebrook and Preston, 1952).

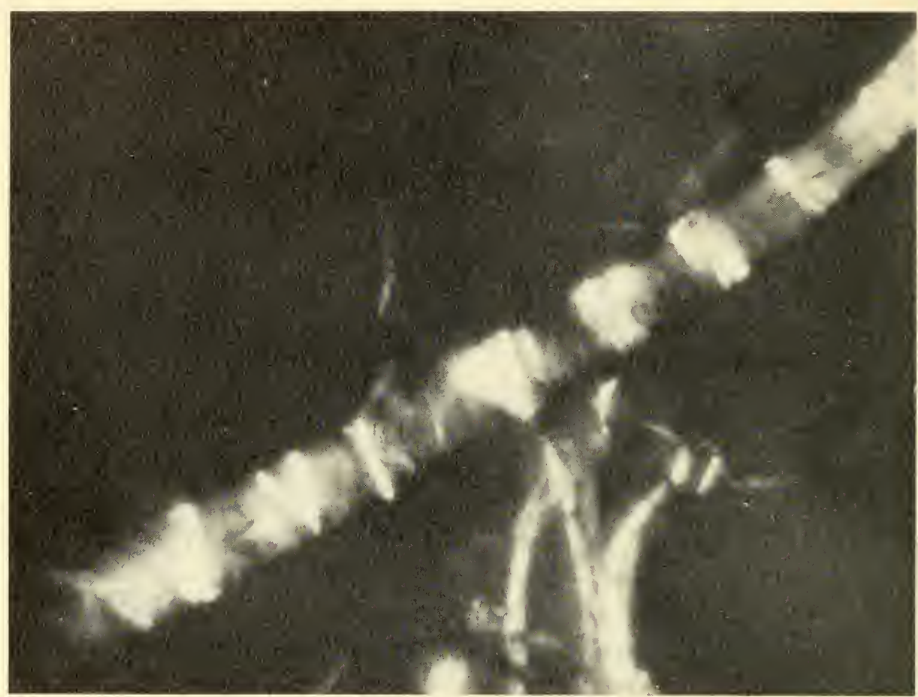

Fig. 3. Antisotropic fiber from bovine epidermis with incomplete polysaccharide sheath, as seen between crossed nicols. The polysaccharide appears bright, the protein core dark. $\times 520$.

The composition of the protein core differs from that in collagen and elastin. Like elastin, the protein is rich in glycine, proline, and valine; it contains some hydroxyproline but less than does collagen. On the other hand, appreciable quantities of acidic and basic amino acids are present, a feature more characteristic of collagen.

It is not, of course, certain that the cellulose is synthesized in situ; the conclusion does, however, seem inescapable that the protein, a degenerative product of collagen, is involved in at least the orientation of the cellulose. 


\section{The Cellulose of Plant Cell Walls}

The structure of cellulose itself is not without significance in this respect. Classically, cellulose is regarded as a polymer of $\beta$-D-glucose residues joined in long chains by $1-4$ links. Over certain parts of their length these chains lie parallel to each other and regularly spaced in a lattice dimensioned as in Fig. 4, which is now known to be only approximately correct (Honjo and Watanabe, 1958; Preston, 1958). The lattice is such as to yield a more or less well-defined diffraction diagram when a beam of $\mathrm{X}$-radiation or electrons is

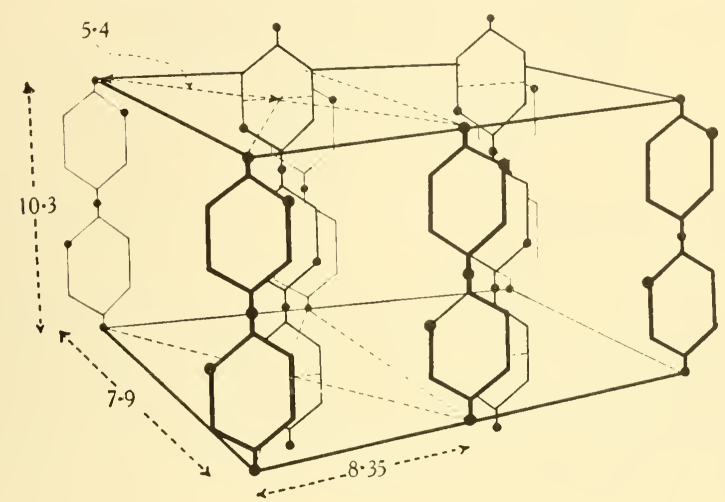

Fig. 4. A unit cell of cellulose after Meyer and Mark. Dimensions are given in Ångström units. The black circles represent oxygen atoms.

passed through it. From such diagrams the spacing between prominent planes in the lattice may be calculated, and particular note may be taken of the spacings at $3.9,5.4$, and $6.1 \AA$ between planes lying parallel to chain direction, and at $2.56 \AA$ from planes lying normal to chain direction (Figs. 4, 5). The lattice occurs, of course, inside the microfibril, which therefore contains a number of molecular chains (of the order of 200) lying parallel to its length.

In general, celluloses isolated from plant cell walls include polysaccharides containing sugars other than glucose, and it is now knowm that these are not always contaminants from the associated amorphous non-microfibrillar components. In a series of analyses controlled by electron microscope observation of wall extracts, Cron- 
Fig. 5. Electron diffraction diagram of Valonia cellulose; orientation correct for comparison with Fig. 4.

shaw et al. (1958) have shown that the microfibrils themselves usually contain sugars other than glucose, and some of the results of these analyses are presented in Table 1. These fully confirm the opinion, which has been gaining ground for a number of years, that cellulose is a mixed polysaccharide. With two species (Valonia and Cladophora) and possibly a third (Chaetomorpha) (Table 1), sugars other than glucose have not been detected. The cellulose involved appears to be a "true" substance in the strict chemical and crystallographic sense, and it has been suggested that it should be distinguished as eucellulose (Myers and Preston, 1959). The structure proposed for cellulose microfibrils is illustrated in Fig. 6. This represents diagrammatically a transverse section of a microfibril (Preston and Cronshaw, 1958; Preston, 1958). The short oblique lines represent the projection of chain molecules in this plane. The central core, represented as a lattice, is crystalline and is surrounded by a cortex in which the arrangement of the chains is less regular. Within this cortex occur chains of glucose residues (solid lines) and of other sugars (broken lines). These may be aggregated together to form larger groups, within which the microfibrils are still distinguishable. This has led Frey-Wyssling (1959) to refer to the single structures as elementary fibrils and to the aggregates as micro- 
fibrils. It seems unnecessary to coin a new terminology in order to include the chance aggregates of variable size, and the term "microfibrils" will be used here as referring to the unit of structure only.

TABLE 1

Composition of Celluloses from Various Sources

\begin{tabular}{|c|c|c|}
\hline Source & & Composition \\
\hline Valonia ventricosa & $\mathrm{S}$ & Glucose \\
\hline Cladophora rupestris & $\mathrm{S}$ & Glucose \\
\hline Chaetomorpha melagonium & $\begin{array}{l}\text { S } \\
\text { W }\end{array}$ & $\begin{array}{l}\text { Glucose } \\
\text { Arabinose }\end{array}$ \\
\hline Enteromorpha sp. & $\begin{array}{l}\mathrm{S} \\
\mathrm{M} \\
\mathrm{M}\end{array}$ & $\begin{array}{l}\text { Glucose } \\
\text { Xylose } \\
\text { Rhamnose }\end{array}$ \\
\hline Ulva lactuca & $\begin{array}{l}\mathrm{S} \\
\mathrm{S}\end{array}$ & $\begin{array}{l}\text { Glucose } \\
\text { Xylose }\end{array}$ \\
\hline Halidrys siliquosa and all Fucales & $\begin{array}{l}S \\
W \\
W\end{array}$ & $\begin{array}{l}\text { Glucose } \\
\text { Xylose } \\
\text { Fucose }\end{array}$ \\
\hline Laminaria digitata and $L$. saccharina & $\begin{array}{l}\mathrm{S} \\
\mathrm{M}\end{array}$ & $\begin{array}{l}\text { Glucose } \\
\text { Uronic acid }\end{array}$ \\
\hline Ptilota plumosa & $\begin{array}{l}\text { S } \\
\text { W } \\
\text { W }\end{array}$ & $\begin{array}{l}\text { Glucose } \\
\text { Galactose } \\
\text { Xylose }\end{array}$ \\
\hline Griffithsia flosculosa & $\begin{array}{l}S \\
W \\
W\end{array}$ & $\begin{array}{l}\text { Glucose } \\
\text { Galactose } \\
\text { Xylose }\end{array}$ \\
\hline Rhodymenia palmata & $\begin{array}{l}S \\
S\end{array}$ & $\begin{array}{l}\text { Glucose } \\
\text { Xylose }\end{array}$ \\
\hline Porphyra sp. & $\mathrm{S}$ & Mannose \\
\hline
\end{tabular}

S. M., W refer to the intensity of the spot on the chromatogram. $\mathrm{S}=$ strong; $\mathrm{M}=$ medium; $\mathrm{W}=$ weak.

Any protein which complexes with this structure must be able to associate with a variety of sugars just as it appears to do in animals. Such a complex will be possible presumably only if the surfaces of the microfibril and the protein have structural features in common, and there is some crystallographic evidence that this may be so. 


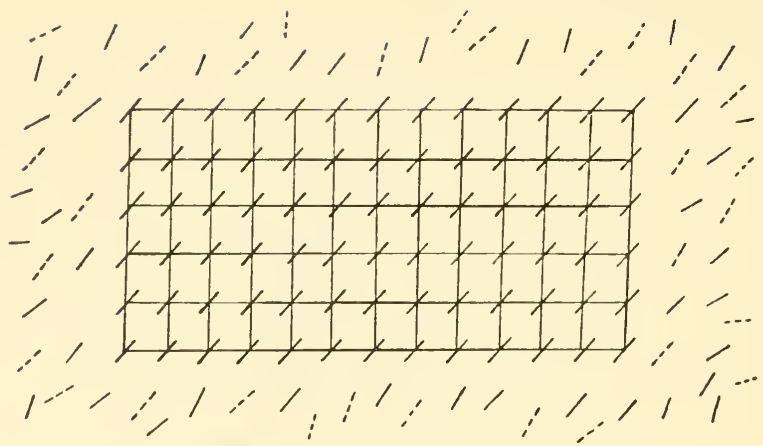

Fig. 6. Diagrammatic representation of a cellulose microfibril in transverse section. Oblique solid lines, projection of polyglucose chains; oblique broken lines, projection of chains containing sugars other than glucose. The central lattice represents the crystalline core, and the longer edges of the core are parallel to the planes of 6.1-Å spacing.

When celluloses are immersed in solutions of salts of heavy metals, the cation is rapidly adsorbed according to a Langmuir isotherm (Belford and Preston, 1959; Belford et al., 1958; Belford et al., 1957, 1959). Parallel bundles of the microfibrils then vield one of two electron-diffraction diagrams, of which one is illustrated in Fig. 7. This is quite different from the diagram yielded by cellulose alone (Fig. 5) and has been interpreted as arising from a twodimensional array of heavy metal ions (Fig. 8). The diagram, and therefore the lattice producing it, is the same whatever metal is used and whatever the source of the cellulose. Two types of array have been calculated, defined by the parameters:

$$
\begin{array}{ll}
\text { Type I } & p=6.15 \AA, q=7.05 \AA, \phi=90^{\circ} \\
\text { Type II } & p=7.32 \AA, q=5.68 \AA, \phi=87^{\circ}-90^{\circ} \text { (variable) }
\end{array}
$$

which explain quantitatively the positions of all the arcs in the diagrams. These parameters do not correspond to those in crystals of the metals, in any metal salt, or in cellulose (see spacings quoted on p. 235 ). Since the only surfaces available for adsorption are those of the microfibrils, this two-dimensional array must lie on these surfaces, and the mutual arrangement of the ions must be governed by the surface. The only possible inference seems to be that the molecular chains lying on the surface of the microfibrils are folded in one of two ways, bringing points of attraction into a lattice de- 
fined by these parameters, in configurations demanded by surface forces.

The significance of these observations is that precisely the same observations have been made also with collagen fibers from the tendon of Achilles. The surfaces of collagen and cellulose microfibrils, therefore, have in common an array of active centers defined by the parameters given above. It is not yet known whether or not other proteins behave similarly; these form the object of further investigations now in progress.

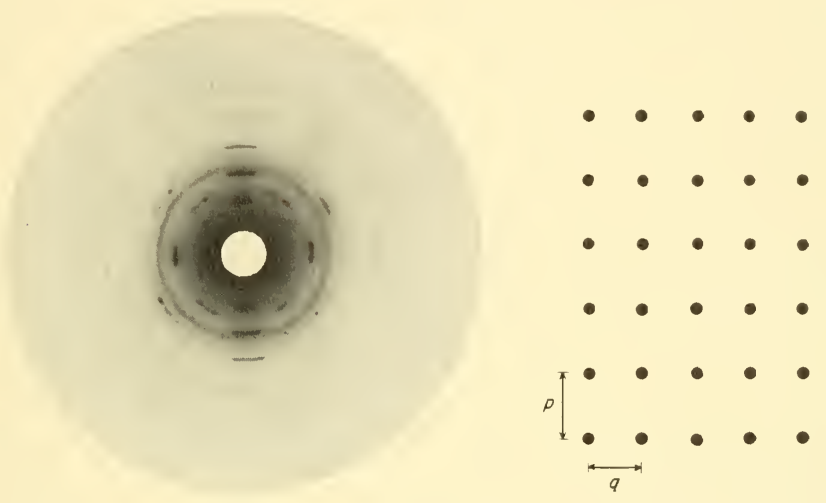

Fig. 7

Fig. 8

Fig. 7. Electron diffraction diagram of the Cu-cellulose complex in Pseudotsuga cellulose; the constituent microfibrils lie parallel to the longer edge of the page. Contrast with Fig. 5 , in which the orientation is the same.

Fig. 8. The two-dimensional array of $\mathrm{Cu}$ atoms presumed to lie on the surface of cellulose microfibrils in the Cu-cellulose complex. The parameter $p$ lies parallel to microfibril length (for values of $p$ and $q$, see text).

\section{The Stability of Protein-Cellulose Complexes in Plants}

Although little specific attention has been given to any mutual stabilization between proteins and polysaccharides in plants, there are indications in the literature of such a stabilization, over and above the frequent statements that high sugar content in a tissue appears to prevent protein degradation. When leaves fall on the soil, die, and are buried, then both the proteins and the polysaccharides break down rapidly under the influence of microorganisms 
and other agencies. It has been shown by Handley (1954), however, that, under the same conditions, leaves which have died on the plant behave very differently. Although the vascular bundles largely disappear, the cells of the epidermis and mesophyll, which might have been considered much less resistant, remain almost intact. Dealing with mor soils, Handley remarks, "Serial sections (of recognizable fragments of litter of Calluna vulgaris-present author) indicated that vascular tissue disappears first, leaving a residue of leaf mesophyll tissue which ultimately becomes an amorphous mass containing mesophyll cell walls apparently coated with some protective material. Similar findings were obtained for . . . beech litter, Norway spruce litter and Abies pinsıpo litter. . . . From serial sections of shoots of Calluna vulgaris, having both dead and living leaves, changes have been observed in the mesophyll tissue when the leaf dies, while still on the living plant, which give the mesophyll tissue the appearance it retains until it becomes amorphous material in the mor. The material appearing . . . would seem to be that which protects the cellulose wall. . . Experimental evidence is put forward to show that the essential part of the change occurring when the leaf dies is a precipitation or stabilisation of cytoplasmic protein."

From this point of view, the recent findings of Steward and Pollard $(1956,1957)$ and Pollard and Steward (1959) are not without significance. They have shown that, when cultures of carrot-root phloem and potato tubers are fed with $\mathrm{C}^{1+}$-labeled proline, the proline can be recovered in a protein which also contains $\mathrm{C}^{14}$ hydroxyproline, clearly produced from the proline. Apart from this, the active carbon appears in no other amino acid, nor does it appear as $\mathrm{CO}_{2 .}$. The protein concerned is therefore stable and does not take part in the normal protein turnover in the plant. Moreover, the protein is not part of any of the cytoplasmic particles which Steward and Pollard examined, and they conclude, therefore, that this protein occurs in the ground cytoplasm. Here, then, is a protein of which at least the proline- and hydroxyproline-containing portion is guarded from the metabolic machinery of the plant. One possibility is that this is the protein which is closely associated with the developing cell wall. This is the only protein extracted from plants to contain hydroxyproline, and recalls immediately the composition of the protein in the cellulose-protein complex described by Hall, and discussed in an earlier section of this paper. 


\section{Electron Microscope Observations of Cell Wall-Cytoplasmic Relations}

There is thus some evidence that cellulose and protein may be associated in plants just as they are now known to be in animal skin. We come now to the electron microscope evidence which seems convincing.

Electron microscope evidence for the interpenetration of walls and the proteins of cytoplasm comes from many sources. Mühlethaler (1950) was the first to show that in primary walls the cellulose microfibrils are twisted round each other, and to state clearly that this could not happen if the wall and cytoplasm were separated by an interface. Similar observations can be made even with secondary walls, and the walls of the seaweeds Valonia, Cladophora, and Chaetomorpha have proved to be particularly revealing (Frei and Preston, 1961).

In these three species, microfibrillar orientation reaches its most perfect state. The walls consist of many lamellae, each normally one or two microfibrils thick, and each consisting of a compact set of parallel microfibrils. These microfibrils lie, moreover, with the planes of 6.1- $\AA$ spacing (Fig. 2) more or less parallel to the wall. The microfibril direction alternates from one lamella to the next through an angle which can be as low as $60^{\circ}$ but is usually rather less than a right angle so that odd lamellae show one orientation and even lamellae the other. In Valonia, in some Cladophora spp., and in some Chaetomorpha spp. (but not all), a third orientation also occurs, much more rarely than the other two. A model of the whole wall of a Valonia vesicle is illustrated in Fig. 9 (Cronshaw and Preston, 1958). This regular alternation from one direction to the other, through an angle which is not the same from point to point in a cell, cannot be ascribed to twinning. It must reflect changes in the proteins at the surface of the cytoplasm, and recalls the somewhat similar arrangement of protein fibrils found in the cuticle of earthworm by Rudall and Reed (see Astbury, 1949). In all lamellae, however, the microfibrils are frequently twisted around each other. Moreover, the switch from one orientation to the other is never clean and abrupt. Microfibrils from one lamella may pass through the next lamella and become part of the next-but-one lamella with the same orientation; microfibrils from one lamella may interweave with those 


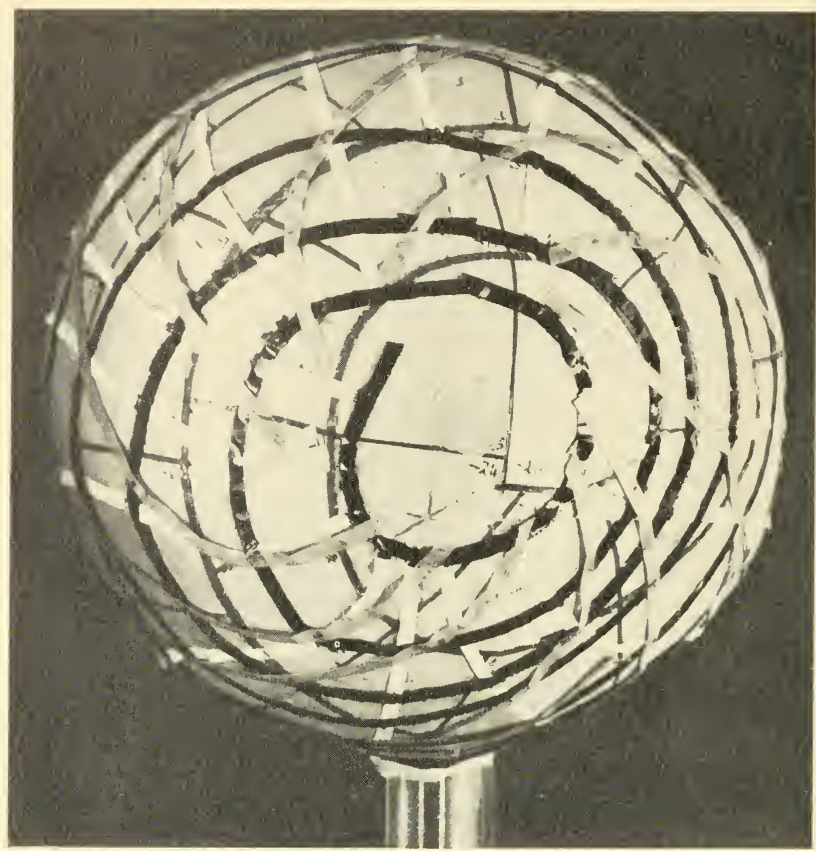

Fig. 9. Model of wall structure in a vesicle of Valonia ventricosa. The black and white tapes represent the run of the two major sets of microfibrils, one in a slow left-hand spiral and the other in a steep left-hand spiral. The gray tape, a fairly steep right-hand spiral, corresponds to the third orientation. One "pole" lies in the center of this figure, the other lying on the far side of the model.

of the next; and microfibrils from one lamella may turn through $90^{\circ}$ and become part of the next lamella (Frei and Preston, 1961). This seems to be clear evidence that the wall and the cytoplasm must be intermixed during microfibril synthesis. Examples of these interweavings are given in Fig. 10.

The strongest evidence for the existence of a cellulose-protein complex comes, however, from examination of the development of a new cell wall over the surface of the spores of these plants, and from an examination of lamellae just being deposited in adult cells (Nicolai, 1957; Nicolai and Preston, 1959; Frei and Preston, 1961; Preston and Kuyper, 1951; Preston et al., 1953). The first wall lamella deposited around a zoospore, gamete, or zygote carries 


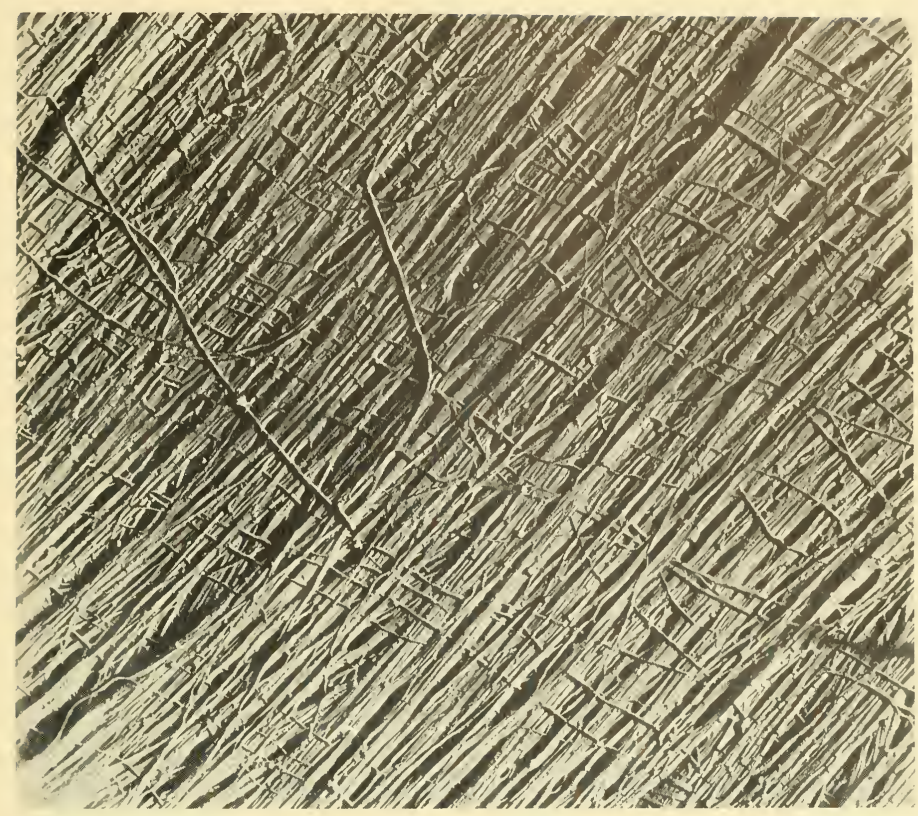

Fig. 10. Electron micrograph of lamellae stripped from the wall of Chaetomorpha melagonium. Note: (1) The apparent "stray" microfibrils are in situ as deposited because they are attached to a lamella at both ends. (2) One set of microfibrils interweaves with the other. The run of the "strays" proves that this interweaving is real and is not due to accidental stripping away of microfibrils. Shadowed Pt. $\times 17,000$.

microfibrils which are oriented at random. In the next one, deposited to the inside of the first (Fig. 11), the microfibrils are oriented transversely to an axis passing through the point of attachment to the substrate. Microfibril synthesis and microfibril orientation seem, therefore, to involve different mechanisms. The microfibrils of this second lamella, which rum in slow spirals round the sporeling, are preceded by the development of coarse bands with similar orientation, which appear to be cytoplasmic (Fig. 11). Microfibrils develop within these bands; they are first observed at a point on what may be called the equator and appear to develop by endsynthesis in such a way that they remain within the coarse bands. In the third lamella, the microfibrils run from pole to pole of the 


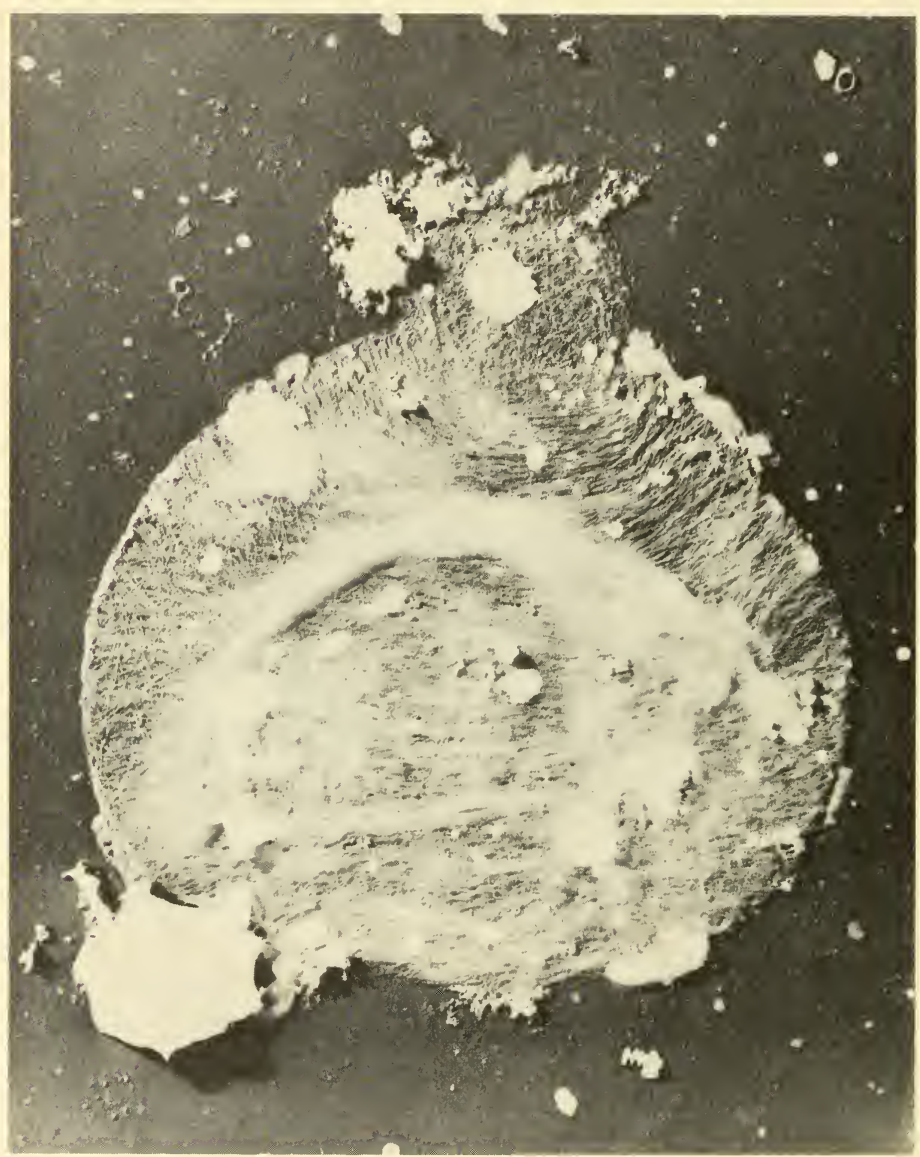

Fig. 11. Electron micrograph of the two-day-old sporeling of Chaetomorpha melagonium. The surface is covered with coarse bands oriented transversely (and therefore in a slow spiral opening from one "pole" at the "base" and closing in toward the sprouting "tip"). Occasional microfibrils can already be seen toward the lower right. 


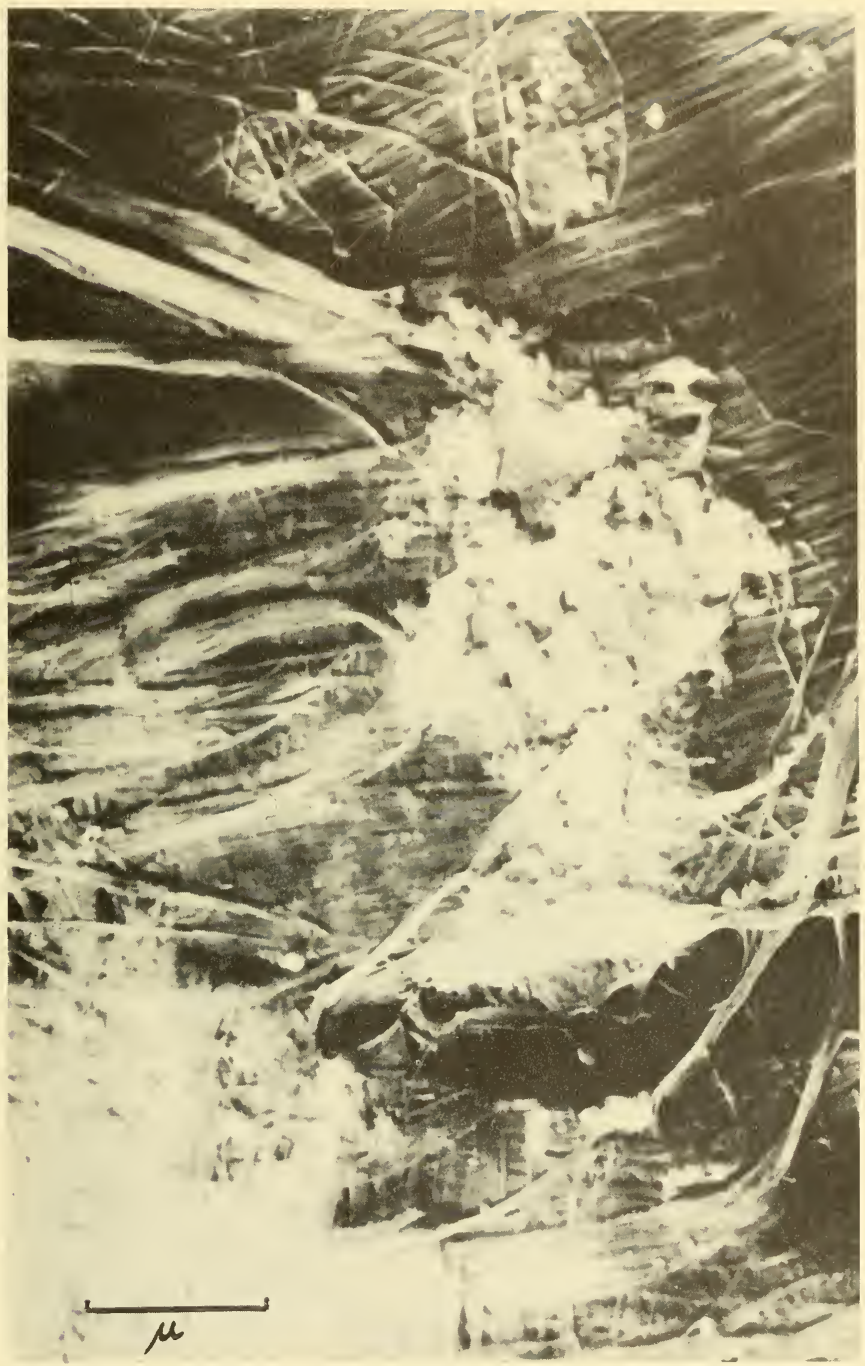

Fig. 12. Electron micrograph of innermost lamella of wall of Valonia ventricosa. The microfibrils appear to radiate from a granular mass. Shadowed Pd-Au. $\times 45,000$. 


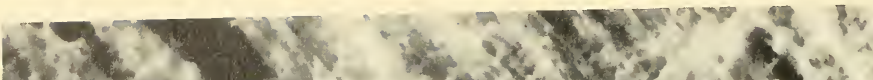

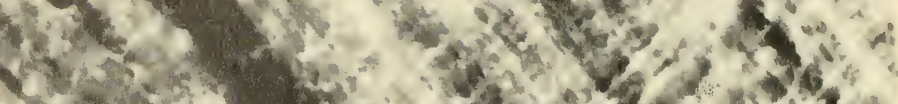
W.

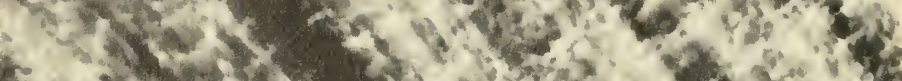

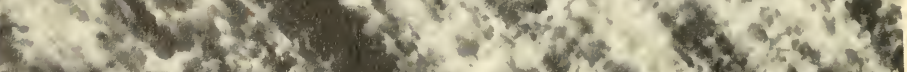

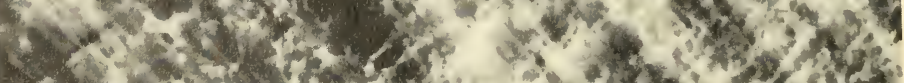

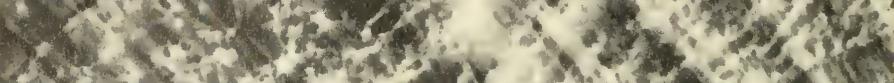

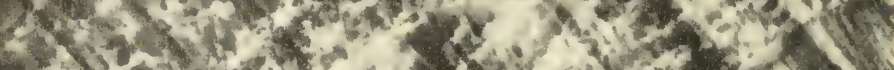

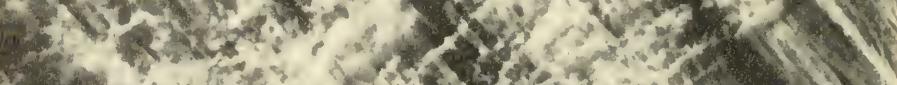

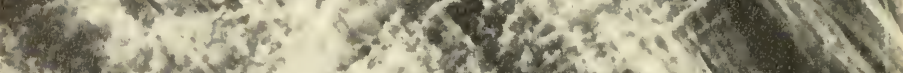

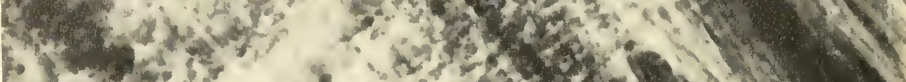

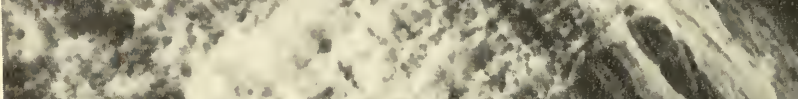

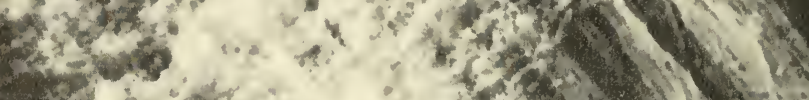

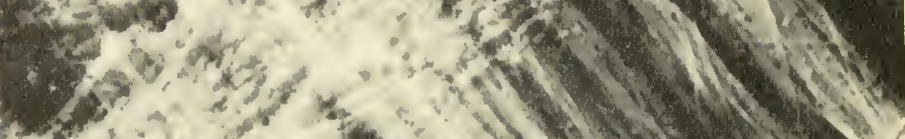

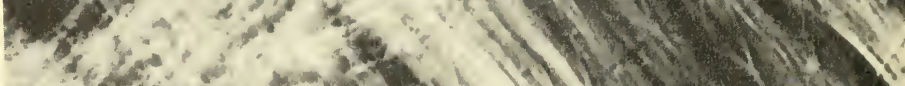

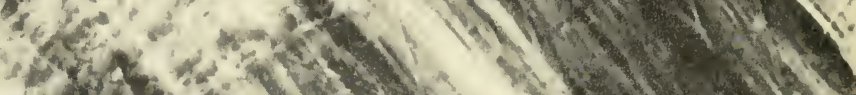

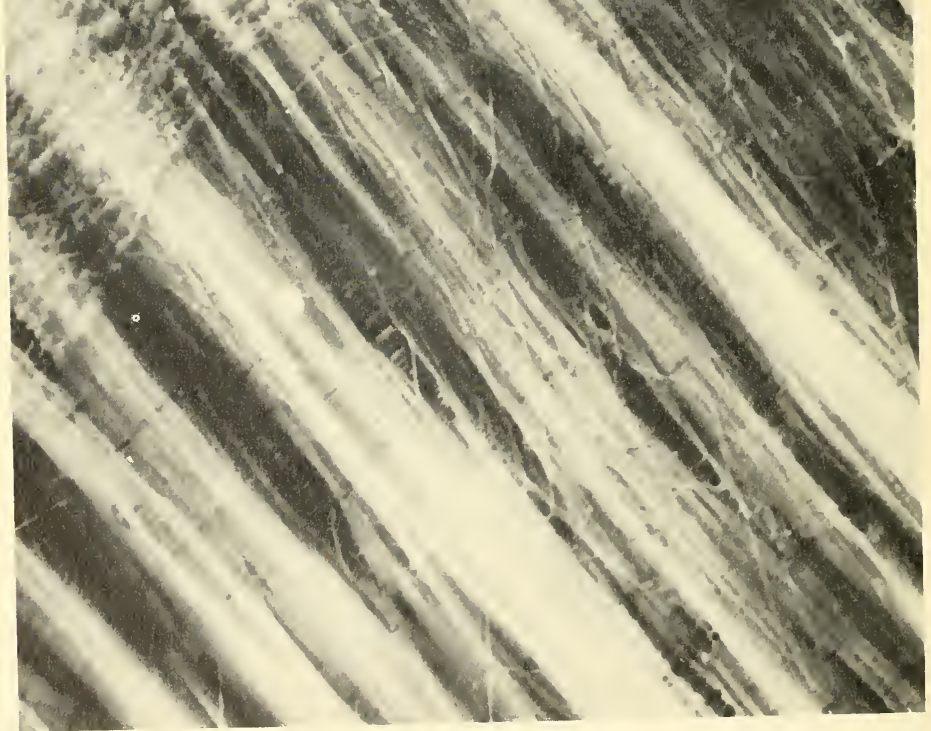


spiral thus produced (and therefore at right angles to the microfibrils of this second lamella) and are again preceded by coarse bands appropriately aligned.

Examination of new lamellae in course of deposition has proved even more critical. The first observations were made on the giant vesicles of Valonia (Preston and Kuyper, 1951; Preston et al., 195.3). The innermost lamella of the wall was stripped off by means of Cellotape, and the two types of critical observation made are illustrated in Figs. 12 and 13. There is no doubt about the latter (Fig. 13); it surely illustrates an undisturbed lamella. In the former (Fig. 12), however, the new lamella is so delicate that some distortion might have occurred. The impression given is that microfibrils project from a cluster of granules each about $500 \AA$ in diameter, and the tentative explanation made at that time was that each cluster represented an "island of synthesis." Microfibrils would then appear to be built by end-synthesis (a method of synthesis later supported by Colvin et al., 1957), association between microfibril and enzyme occurring only at one end. The mechanism of orientation remained obscure, but observations of the type shown in Fig. 13 suggested that orientation occurred in the cytoplasm before the microfibrils of the new lamella were produced.

More recent observations of Chaetomorpha, using more delicate techniques, are more convincing on this latter point (Frei and Preston, 1961). In these, the filaments are either fixed directly in an osmium fixative or plasmolyzed in $0.5 \mathrm{M}$ sucrose before fixation, but in either case the appearance to be described is the same. The lamellae are then teased off in water, using fine needles under a binocular microscope. Fig. 14 illustrates the type of observation to which attention is now called. This is a frequent but not a constant observation; more frequently the lamella is so densely covered by cytoplasm that no details are visible. Occasionally the lamella is completely free of cytoplasm. Fig. 14 shows clearly, however, aggregates of dense granular bodies lying in files on the wall lamella, the long axes of the files lying approximately at right angles to the microfibrils, i.e., in the direction which the fibrils of a new lamella would take. It is to be emphasized that the lamella observed here

Fig. 13. Electron micrograph of innermost lamella of wall of Valonia ventricosa. Granular material, evidently of cytoplasmic origin, has taken up an orientation which the next layer of microfibrils would have adopted. Shadowed Pd-Au. $\times 22,500$. 


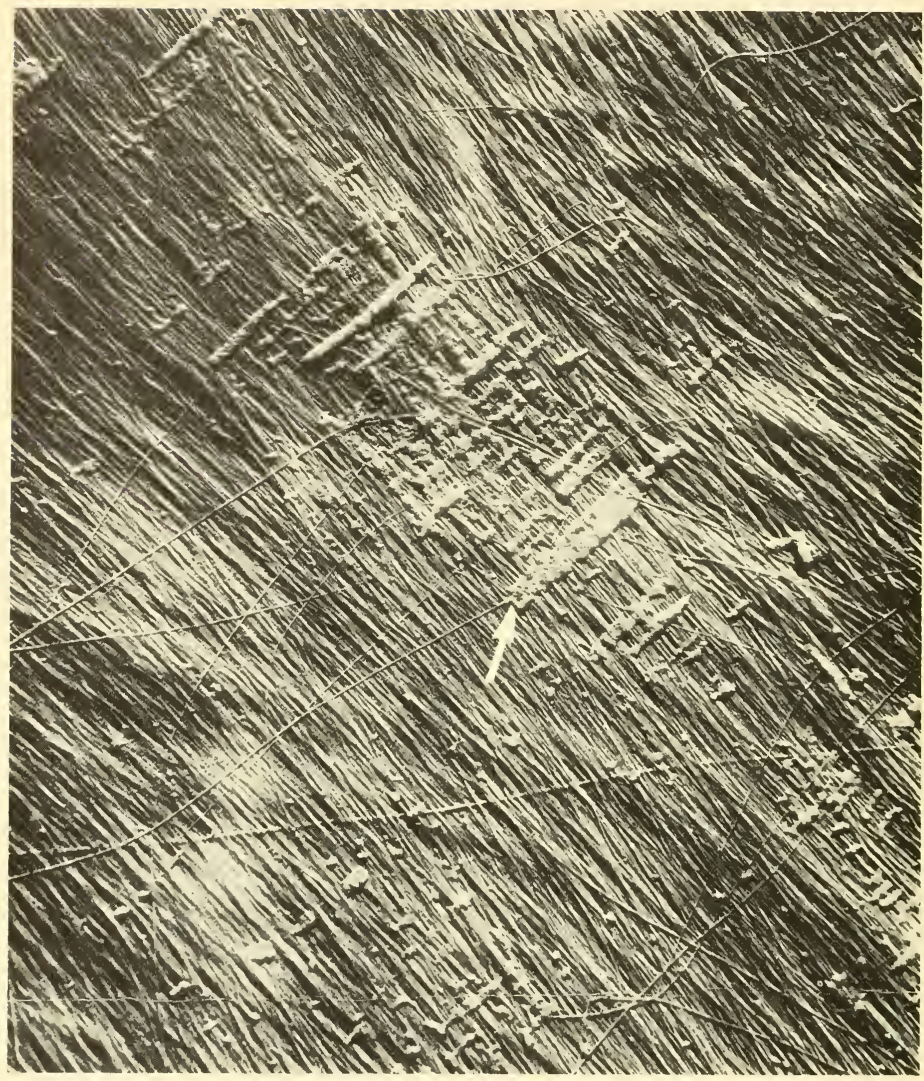

Fig. 14. Electron micrograph of the innermost layer of Chaetomorpha melagonium wall. Note the linear files of aggregated granules oriented at right angles to the microfibrils of the last lamella deposited and therefore parallel to those of a lamella about to be deposited. These are the remnants of a much more extensive system. Shadowed Pt. $\times 20,400$.

is the innermost one lying next to the cytoplasm, and very few microfibrils of the next lamella have yet been synthesized. These files cannot therefore represent debris lying between microfibrils which have been stripped away. When microfibrils of the new lamella do appear, they appear to be embedded in this material and sometimes 
to project from one end. One example of this, marked by an arrow, is visible in Fig. 14.

When similar preparations are made of the innermost lamellae of cells which have previously been plasmolyzed, these oriented cytoplasmic aggregates remain attached to the lamella. They are therefore more closely attached to the wall than is the bulk of the cytoplasm, which retracts on plasmolysis. A new wall lamella does not develop under these conditions, presumably because of the withdrawal of the supply of raw materials necessary. The new cytoplasmic surface does produce a new cell wall, but the microfibrils are not oriented. When, however, filaments plasmolyzed in, say, $0.8 M$ sucrose are returned to sea water, they continue to grow normally. After a few days in sea water, wall layers similar to that illustrated in Fig. 14 can be found deep in the wall, covered by more recent wall lamellae with the usual alternations in microfibril orientation. A cytoplasmic surface, withdrawn from the wall and at that time producing microfibrils oriented at random, can thus reconstitute the whole orientation mechanism.

\section{Discussion and Conclusions}

Although, so far as the writer is aware, there is no direct biochemical evidence of close association between cellulose and protein in plant cell walls, the morphological observations and structural determinations referred to above, together with the evidence from parallel conditions in animals, however, is strongly suggestive. It would appear to be established that microfibrils are synthesized by what appears to be end-synthesis and that, at least during and immediately after synthesis, the part of the microfibril concerned is embedded in an aggregate of granules which may be enzyme complexes and therefore largely protein. Microfibrils are occasionally observed, on the surface of new wall lamellae, which have long tapering ends rumning down to the limits of visibility in the electron microscope. It would seem, therefore, possible that synthesis occurs over long lengths of microfibril and is end-synthesis of individual molecular chains rather than of whole microfibrils.

It would certainly be premature to attempt to give even a purely morphological picture of the cellulose-protein complex during synthesis. Appearances do suggest, however, that a cytoplasmic surface which is to produce a wall lamella first becomes organized into 
coarse bands oriented with respect to the cell axis. At some point in the band-which presumably already contains a microfibril endsynthesis begins. As this proceeds, the microfibril end moves forward through the coarse band and new parts of the band take over synthesis. At this time, the coarse band has a central core of cellulose and an outer sheath of protein-the reverse of the condition in animal skin. It is not clear whether the diameter of the microfibril (which varies only within rather narrow limits) is a function of the size of the protein aggregates or is controlled thermodynamically as suggested by the work of Fïrth (1955).

Eventually the cellulose-protein complex must disintegrate, so that the microfibril lies free on the wall surface.

When these protein aggregates take up a new alignment in preparation for the deposition of the next lamella, it would then follow that synthesis of the new lamella could begin only at microfibril ends of the lamellae just completed. This would explain the many interweavings between microfibrils of different lamellae. This does not in any sense "explain" the specific orientations of cellulose microfibrils, but merely puts the mechanism one step further back into the cytoplasm. In spite of earlier criticisms of orientation by protoplasmic streaming, it seems possible that in some plants there is some association between streaming and orientation and that this is achieved through orientation of proteins (Probine and Preston, 1958). It is not at the moment possible to decide whether streaming is involved in the cases discussed in this paper.

These considerations refer specifically, of course, only to those few algae in which microfibrillar orientation is so perfect that the necessary critical observations have been made. It seems unlikely, however, that the basic principles will be different in other plants. The present situation is, in brief, that such evidence as is available points toward the type of mechanism which may be involved in wall synthesis. Further progress clearly demands a thorough biochemical study of the proteins in or near the cytoplasmic surface.

\section{References}

Akiya, A., and M. Tomoda. 1956. J. Pharm. Soc. Japan 76: 1092. (Cited in Hall et al., 1960.)

Allsopp, A., and P. Misra. 1940. The constitution of the cambium, the new wood and the mature sapwood of the common ash, the common elm and the Scotch pine. Biochem. J. 34: 1078-1084. 
Astвuny, IV. T. 1949. The structure of biological tissues as revealed by x-ray diffraction analysis and electron microscopy. Brit. J. Radiol. 22: 335-365.

Belford, D. S., A. Myers, and R. D. Preston. 1958. Electron diffraction study of adsorbed metal ions on the surface of cellulose microfibrils. Naturc 181: 15161518.

Belford, D. S., and R. D. Preston. 1959. Ordered adsorption of metal ions on cellulose. Biochim. et Biophys. Acta. (In press.)

Belford, D. S., R. D. Preston, C. D. Cook, and E. 11. Nevard. 1957. Timber preservation by copper compounds. Nature 180: 1081-1083.

Belford, D. S., R. D. Preston, C. D. Cook, and E. H. Nevard. 1959. The impregnation of timber by water-borne preservatives. I. General survey. J. Appl. Chem. (London) 9: 192-200.

Bishop, C. T., S. T. Bayley, and G. Setterfield. 1958. Chemical constitution of the primary cell walls of Avena coleoptiles. Plant Physiol. 33: 283-289.

Burton, D., D. A. Hall, M. K. Keech, R. Reed, H. SAxl, R. E. Tunbridge, and M. J. Wood. 1955. Apparent transformation of collagen fibrils into 'elastin." Nature 176: 966-969.

Colvin, J. R., S. T. BAyley, and M. Beer. 1957. The growth of cellulose microfibrils from Acetobacter xylinum. Biochim. et Biophys. Acta 23: 652-653.

Consden, R., and R. Bird. 1954. The carbohydrate of connective tissue. Nature 173: 996-997.

Cronshaw, J., A. Myers, and R. D. Preston. 1958. A chemical and physical investigation of the cell walls of some marine algae. Biochim. et Biophys. Acta 27: 89-103.

Cronshaw, J., and R. D. Preston. 1958. A re-examination of the fine structure of the walls of vesicles of the green alga Valonia. Proc. Roy. Soc. (London) B 148: $137-148$.

Dische, A., and G. Zelemenis. 1955. A.M.A. Arch. Ophthalmol. 54:528. (Cited in Hall et al., 1960.)

Dische, Z., and E. Borenfreund. 1954. Am. J. Ophthalmol. 38: 165. (Cited in HALL et al., 1960.)

Dische, Z., A. Danilczenko, and G. Zelemenis. 1958. In Chemistry and Biology of Mucopolysaccharides. Ciba Foundation. J. \& A. Churchill Ltd., London.

Dohlman, C. H., and E. A. Balazs. 1955. Arch. Biochem. Biophys. 57: 44. (Cited in HALL et al., 1960.)

Frei, E., and R. D. Preston. 1961. Proc. Roy. Soc. (London) B. (In Press.)

Frey-IVyssling, A. 1959. Die Pflanzliche Zellwand. Springer Verlag, Berlin.

Fürth, R. 1955. Fundamental lengths in physics and biology. Exptl. Med. Surg. 13: $17-21$.

Grassman, W., and H. Schleich. 1935. Über den Kohlenhydratgehalt des Kollagens II. Biochem. Z. 277: 320-328.

Gross, J., J. H. Highberger, and F. O. Schirtt. 1952. Proc. Soc. Exptl. Biol. Med. 80: 462. (Cited in Hall et al., 1959.)

Hall, D. A. 1955. The reaction between elastase and elastic tissue. Biochem. J. 59: 459-465.

Hall, D. A., F. Happey, P. F. Lloyd, and H. Saxl. 1960. Oriented cellulose as a component of mammalian tissue. Proc. Roy. Soc. (London) B 151: 497-516.

HALL, D. A., and R. REED. 1957. Hydroxyproline and thermal stability of collagen. Nature 180: 243.

Hall, D. A., R. Reed, and R. E. Tunbridge. 1952. Structure of elastic tissue. Nature 170: 264-266.

Hall, D. A., and H. SAxl. 1961. Submitted to Proc. Roy. Soc. (London) B.

Handley, IV. R. C. 1954. Mull and mor formation in relation to forest soils. Forestry Commission Bull. No. 23. H. M. Stationery Office, London.

Honjo, G., and M. Watanabe. 1958. Examination of cellulose fibre by the lowtemperature method of electron diffraction and electron microscopy. Nature 181: 326 . 
JACKson, D. S. 1953. The nature of collagen-chondroitin sulphate linkages in tendon. Biochem. J. 56: 699-703.

KeECH, M. K. 1958. Ann. Rheumatic Diseases 17: 23. (Cited in Hall et al., 1960.)

Middlebrook, Mavis J., and R. D. Preston. 1952. Spiral growth and spiral structure. III. Wall structure in the growth zone of Phycomyces. Biochim. et Biophys. Acta 9: 32-48.

MühlethaleR, K. 1950. Elektronmikroskopische Untersuchungen über den Feinbau und das Wachstum in Mais- und Haferkoleoptilen. Ber. schweiz. botan. Ges. 60: 614 .

Myers, A., and R. D. Preston. 1960. Fine structure in the red algae. II. The structure of the cell wall of Rhodymenia palmata. Proc. Roy. Soc. (London) B. (In press.)

Myers, A., R. D. Preston, and G. W. Ripley. 1956. Fine structure in the red algae. 1. X-ray and electron microscope investigation of Griffithsia flosculosa. Proc. Roy. Soc. (London) B 144: 450-459.

Nicolal, E. 1957. Wall deposition in Chaetomorpha melagonium (Cladophorales). Nature 180: 491-492.

Nicolai, E., and R. D. Preston. 1959. Cell wall structure in the Chlorophyceae. III. Differences in structure and development in the Cladophoraceae. Proc. Roy. Soc. (London) B. 151: 244-255.

Northcote, D. H., K. IV. Goulding, and R. W. Horne. 1958. The chemical composition and structure of the cell wall of Chlorella pyrenoidosa. Biochem. J. 70: 391-397.

Pollard, J. K., and F. C. Steward. 1959. The use of $\mathrm{C}^{14}$-labelled proline by growing cells; its conversion to protein and hydroxyproline. J. Exp. Botany 10: 17-32.

Preston, R. D. 1947. The fine structure of the wall of the conifer tracheid. II. Optical properties of dissected cells in Pinus insignis. Proc. Roy. Soc. (London) B 134: 202-218.

Preston, R. D. 1951. Fibrillar units in the structure of native cellulose. In The size and shape factor in colloidal systems. Discussions Faraday Soc. No. 11: 165-170.

Preston, R. D. 1952. Molecular Architecture of Plant Cell Walls. Chapman and Hall, Ltd., London.

Preston, R. D. 1958. Wall organization in plant cells. Ann. Rev. Cytol. 8: 33-60.

Preston, R. D., and J. Cronshaw. 1958. Constitution of the fibrillar and nonfibrillar components of the walls of Valonia ventricosa. Nature 181: 248-250.

Preston, R. D., and B. Kuyper. 1951. Electron microscopic investigations of the walls of green algae. I. A preliminary account of wall lamellation and deposition in Valonia ventricosa. J. Exp. Botany 2: 247-255.

Preston, R. D., E. Nicolai, and B. Kuyper. 1953. Electron microscopic investigations of the walls of green algae. 1I. The cytoplasm-wall relationship in freezedried Valonia macrophysa. J. Exp. Botany 4: 40-43.

Preston, R. D., and A. B. Wardrop. 1949. The submicroscopic organisation of the walls of conifer cambium. Biochim. et Biophys. Acta 3: 549-559.

Probine, M. C., and R. D. Preston. 1958. Protoplasmic streaming and wall structure in Nitella. Nature 182: 1657-1658.

SAXL, H. 1957. Gerontologia. 1: 142. (Cited in HaLl et al., 1960.)

Steward, F. C., and J. K. Pollard. 1956. The amino acid composition of the total protein of growing and non-growing tissues. Plant Physiol. 31 : ix.

Steward, F. C., and J. K. Pollard. 1957. Proline and hydroxyproline in the protein of growing cells. Ibid. 32 : lii-liii.

Thimann, K. V., and J. Bonner. 1933. The mechanism of the action of the growth substance of plants. Proc. Roy. Soc. (London) B 113: 126-149.

Wood, G. C. 1954. Some tensile properties of elastic tissue. Biochim. et Biophys. Acta 15: 311-324.

Yu, S. Y., and H. T. Blumenthal. 1958. J. Gerontol. 13:366. (Cited in Hall et al., 1960.) 


\section{DISCUSSION}

\section{V. Edds, Jr., R. D. Preston}

Dr. Ends (Brown University): How do you separate the lamellae of the Valonia cell wall in preparation for electron microscopy?

Dr. Preston: The separation of lamellae of the Valonia cell wall is comparatively easy. A square is cut out of the wall and is left to dry down on a glass slide. A piece of Cellotape is then pressed on the wall and, on peeling the Cellotape from the slide, some of the wall remains on the Cellotape and some on the glass. The process can be repeated until a thin lamella is left either on the Cellotape or on the glass. This lamella can then be separated from the glass by flotation in water, or from the Cellotape with appropriate solvents. A more reliable method, however, is to mount a piece of wall so that the part of it to be examined overlaps a hole in a piece of adhesive paper. Stripping by Cellotape then leaves the final lamella projecting over the hole without ever coming into contact with either glass or Cellotape.

Alternatively (and better), the lamella can be teased off in water, using fine steel needles. This requires considerable manipulative skill, but produces a preparation with a minimum of distortion and without any contamination by Cellotape. 



\section{Index}

Aggregation, 7, 19-20, 21-24, 33-37, 5384

Amino acid analysis, 222; see also specific amino acids

Antigenic patches, 164

Apatite, 54, 55, 69, 83

Arbacia, 169

Aster, 161-162, 167-168

ATP, 24, 51, 52

Autoradiography, 126

Axon, 140-141

Axoplasm, 141

Bacteriochlorophyll, 185

Barker, S. B., 83

Beck, L. V., 83

Bergeron, J. A., 179-203, 228

Bimolecular leaflet, 139-140, 152

Bond

disulfide, 11-12, 167-169

hydrogen, 5-7, 20-21

ionic, 5-7

phosphate, 179-180

Bone, 53-84

Bud scar, 216

Budding, 217, 225

Calcification, 53-84

Candida, 225

Carbohydrates, 5

Carotenoid, 185

Cartilage, 53-84

Casein, 11

Cell

division, 161-178, 225

wall, 205, 206, 229, 241

Cellulose, 205-228, 229, 236

Cementum, 55

Centrosomes, 161

Charge distribution, $30,32,52,62$

Chitin, 83, 209

Chlamydomonas, 95, 99

Chlorophyll, 85-112, 150

Chloroplast, 85-112, 150, 180

Chloroplastin, 100-103, 199

Chondroitin sulfate, 233

Chròmatium, 180

Chromatophore, 86, 179-204

Chromosome, 15
Chymotrypsin, 41

Collagen, 14, 19-52, 53-84, 232

Collagenase, 44

Complex

antigen-antibody, 164

cellulose-protein, 205-228, 229, 233

chitin-protein, 17

chlorophyll-protein, 85-112

glucomannan-protein, 205-229

lipoprotein, 115, 118

polysaccharide-protein, 205,214, 220, 232

Conchiolin, $83 \mathrm{ff}$.

Covalent bonds, 4

Cross-linking, Ca-phosphate, 12

Cryofixation, 116, 118, 123

bromine, 138

osmium, 131, 141

Crystalline ordering, 17

Crystallization, 55-84, 105-109

Cytochrome, 86

Dentin, 55

Ducoff, H., 227

Edds, M. V., Jr., 176, 203, 253

Elastoidin, 82

Electron-spin resonance, 151

Elodea, 95

Energy, interaction, 13

Energy transfer, 150

Entropy, 13

Enzyme reactions, hydrolytic, 13

Euglena, 90, 98, 101

Falcone, G., 205-227

Fatty acid, 17

FDNB, 69 ff., 83

Fernández-Morán, H., 113-159

Fibril formation, 9, 11, 19-52

Fibrillar system, 161-178, 229-253

Fibrogenesis, 9, 11, 19-52

Fibrous long-spacing, 28

Forces

intermolecular, 68

London-van der Waals, 7, 17

long-range, $5,12,15$

short-range, 5,7

Free radicals, 116, 120 
Freeze-drying and freeze-substitution, 117

Fuller, R. C., 179-202

Gel-diffusion technique, 163

Giese, A. C., 112, 158, 177, 203

Glimcher, M. J., 53-84

Glucan, 206

Glucomannan, 207

Glycoprotein, 28, 32

Green, J. W., 82, 227

Ground substance, 77

Group

carboxyl, 71

disulfide, 167

guanidino, 71

phosphorus, 12

polar, 21

sulfhydryl, 167

Group-blocking and group-substitution, 74

Helices, 5, 20, 22, 37, 40

Helium, liquid, 116, 119-122

Hexosamine, 232

Hodge, A. J., 19-52, 112

Hydroxylysine, 69, 75, 83

Hydroxyproline, 240

Ice-crystal formation, 129-131

Insulin, 9

Interaction

charge, 13

energy, 5,7

long-range, $5,12,15$

molecular, 3, 4, 8, 19-52, 53-84

short-range, 5,7

sonic, 25,37

Johnson, W. H., 51

Kessler, G., 205-227

Lamellae, 4, 8, 13, 85-112, 113-161, 241 basement, 14

Liesegang phenomena, 105

Lilium, 168

Low-temperature preparation, 115, 117

Lysine, 69, 75, 83

McElroy, W. D., 17, 51

Mannan, 207

Matrix, organic, 53-84

Mazia, D., 161-176

Membrane

double, $114 \mathrm{ff}$.

unit, 114, 142
Mercaptoethanol, 167

Metastable equilibrium, 54, 55, 62, 117

Micelle, 11

Microfibril, 229, 236, 237, 243

Mineralization, 53-84

Mitochondria, 150

Mitotic apparatus, 161-178

Molecular

fingerprint, 24

interaction, 3, 4, 8, 19-52, 53-84

organization, 150

recombination, 8

Monolayer, 91, 198

Monomolecular films, 8

Myelin, 113, 114, 126, 127, 134, 138, 150,151

Nickerson, W. J., 17, 205-228

Nucleation, 10, 17, 19, 55, 62, 82

Nucleic acids, 5, 86

Orthogonal pattern, 14

Oyster, 83

Paracrystalline state, 108-109, 148-150

Paramyosin, 34, 51

Pattern, diffraction, 20, 22

Pepsin, 41, 44

Periodicity, 19-54, 105-109

Phase transformation, 117

Phospholipid, 185

Phosphorylation, 69

Photochemical organelle, 182, 188

Photophosphorylation, 187

Photopigment, 144, 150

Photopolymerization, 118

Photoreceptor, 85, 113, 142, 146, 150

Photosynthesis, 9, 85-112, 179-204

Photosynthetic pigments, 87,181

Phytol, 92, 198

Polymerization, 28, 37, 38, 43, 52, 118

Polypeptides, 5, 19, 40, 62

Polysaccharide, 186, 206, 222

Porphyrin, 92, 95, 198

Preston, R. D., 227, 229-253

Prosser, C. L., 52, 83

Protease, 41, 45

Protein, 5, 19-52, 53-84, 161-178, 185, 205-228, 229-253

Protein disulfide reductase, 225

Protofibrils, 34, 62

Retinal pigment, 146

Retinal rod, 101, 126, 142, ]45

Rhodopsin, 101

Rhodospirillum, 99, 179

Richards, A. G., 17, 83 
Schmitt, F. O., 19-51

Schwann cell, 114, 139

Segment long-spacing, 24

Semiconductor, 98, 151

Sherman, F. G., 52

Sol-gel transformation, 169

Specificity, $3 \mathrm{ff}$., 62

Spindle, 161-178

Strongylocentrotus, 168

Symmetry, 68

Trillium, 168

Tropocollagen, 19-52
Trypsin, 41, 44

Tyrosine, 47, 48

Uronic acid, 232

Valonia, 237, 241

Van Holde, K. E., 81

Water, 4, 9, 12, 13, 17, 151

Waugh, D. F., 3-17, 203

Went, H. A., 161-177

Wolken, J. J., 85-112

Yeast cell wall, 229-253 








